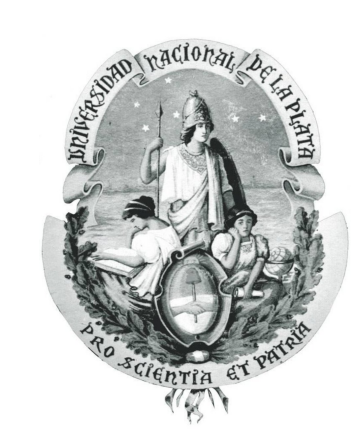

\title{
VIENTOS IMPULSADOS POR RADIACIÓN en Estrellas Supergigantes B EN RotACIÓN
}

Roberto Oscar José Venero

Directora: Dra. Lydia S. Cidale $]^{1}$

Codirector: Dr. Michel Curê2

Tesis PRESENTAdA PARA OPTAR POR EL GRADo DE DoCTOR EN Astronomía

\author{
Universidad Nacional de La Plata \\ Facultad de Ciencias Astronómicas y Geofísicas
}

\section{La Plata, Argentina \\ - MAYO DE 2017 -}

\footnotetext{
${ }^{1}$ Facultad de Ciencias Astronómicas y Geofísicas e Instituto de Astrofísica La Plata, CCT La Plata - CONICET-UNLP.

${ }^{2}$ Instituto de Física y Astronomía - Facultad de Ciencias - Universidad de Valparaíso - Valparaíso, Chile.
} 


\section{Resumen}

En las estrellas tempranas, la transferencia de momento del campo radiación al material que compone sus atmósferas, es el mecanismo principal que impulsa a sus intensos vientos estelares. Esta transferencia se produce, preponderantemente, en las transiciones atómicas que originan a las líneas espectrales. A partir de esta idea se ha desarrollado la teoría de vientos impulsados por radiación en líneas espectrales, que ha brindado notables avances en la interpretación de las observaciones y aportado importantes herramientas de diagnóstico, tales como la relación Momento del Viento - Luminosidad, que permite determinar distancias galácticas y extragalácticas a estrellas masivas por medios espectroscópicos. La teoría describe a la fuerza de radiación que impulsa al viento, por medio de tres parámetros básicos $(k$, $\alpha$ y $\delta$ ), relacionados con la capacidad del medio para absorber momento y el estado de excitación/ionización del material.

La incorporación de la rotación a las ecuaciones hidrodinámicas que describen la velocidad y la densidad a lo largo del viento, genera tres clases diferentes de soluciones: las soluciones rápidas, descritas por la teoría estándar, y dos tipos de soluciones lentas llamadas $\Omega_{\text {lenta }} \mathrm{y}$ $\delta_{\text {lenta }}$, que caracterizan a vientos más densos, con velocidades terminales más bajas que los de régimen rápido. La solución $\Omega_{\text {lenta }}$ se presenta en estrellas en alta rotación, mientras que la solución $\delta_{\text {lenta }}$ surge para ciertas condiciones de ionización del viento.

En esta tesis se estudia la distribución de estas soluciones en el espacio de parámetros de la fuerza de radiación, para estrellas supergigantes B con diferentes tasas rotacionales. El objetivo de este análisis es delimitar los dominios de las soluciones, con el fin de derivar las condiciones físicas imperantes en los vientos complejos de estas estrellas. Se busca, además, evaluar la aplicabilidad de estas soluciones para modelar los vientos, con el fin de reducir las discrepancias que se encuentran entre las predicciones de la teoría estándar y las observaciones.

Para desarrollar el estudio se usan dos códigos de cálculo: el código HYDWIND, para obtener las soluciones a partir de las ecuaciones hidrodinámicas, en combinación con el código FASTWIND, que resuelve el transporte de radiación en medios en movimiento fuera de equilibrio termodinámico local. Para adaptar el código FASTWIND, se incorpora un tratamiento novedoso de las inversiones de poblaciones atómicas tanto en la aproximación de Sobolev con continuo, como en el marco de referencia solidario al medio (comoving frame). Ambas contribuciones permiten mejorar la operatividad de FASTWIND, en especial, para las estrellas supergigantes B tardías.

Usando ambos códigos se calculan líneas espectrales originadas en el viento y se analiza la dependencia de la forma de sus perfiles para las distintas soluciones mencionadas. Como corolario, se ajusta los perfiles sintéticos para las soluciones lentas a perfiles observados de supergigantes B, demostrando la aplicabilidad de estas soluciones. Para finalizar, se revisan las relaciones más relevantes de la teoría estándar, para evaluar la importancia de las soluciones lentas en los vientos rotantes de las estrellas tempranas. 
0. Resumen 


\section{Dedicatoria}

Para Agustín, Salvador y Máximo. 
0. Resumen 


\section{Agradecimientos}

Quiero expresar mi agradecimiento a mucha gente que me ayudó en este trabajo:

A mis compañeros de oficina, por darme su apoyo y su confianza. Son muchos, no los nombro pero están aquí. También a los colegas trasandinos, Ignacio y Catalina. A Michaela Kraus por su gentil colaboración.

A Joachim Puls, quien me recibió con los brazos abiertos en mis tiempos confusos, con su sinceridad y la precisión de un orfebre, me enseño a superarme, dando por sentado que yo podía hacerlo.

A Maxi Haucke, quien, con su ejemplo y calidez, me enseño a avanzar siempre para adelante, a superar el miedo a avanzar, que era mucho.

A Michel Curé, su corazón enorme es una fuente de generosidad inagotable y de entusiasmo. Compartiendo su pasión, me enseñó a trabajar con alegría.

A Lydia Cidale, un edificio hecho de una enorme paciencia hacia mi impericia y mis dudas, llena de empuje y energía, de una memoria prodigiosa y una profunda capacidad para atar ideas. Lydia, que me esperó cuando llovía y me abrió su refugio.

A María Laura, mi compañera, mi alma. Ella ya sabe.

A Agustín, Salvador y Máximo, porque dejé muchas noches de leerles historias, por dedicarme a esta tesis y vi su resignada comprensión. Aquí voy, chicos. A recuperar el tiempo perdido. 
0. Resumen 


\section{Índice general}

Resumen iii

1. Los Vientos Estelares en las Estrellas Masivas 1

1.1. Los vientos estelares . . . . . . . . . . . . . . . . . . . . 1

1.1.1. Evidencias de los vientos estelares . . . . . . . . . . . . . . 2

1.1.2. Relevancia de los vientos estelares . . . . . . . . . . . . 7

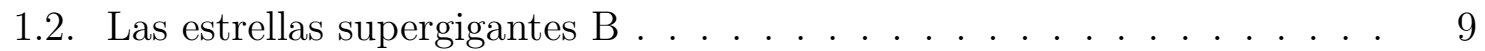

1.2.1. Grupos de supergigantes B . . . . . . . . . . . . . . . 10

$1.2 .2 . \quad$ Estado evolutivo $\ldots \ldots \ldots \ldots \ldots$

1.2.3. Parámetros fundamentales de las supergigantes B . . . . . . . 15

1.2.4. Parámetros globales de los vientos estelares . . . . . . . . . 18

1.2.5. Estimación de $\dot{\boldsymbol{M}}$. . . . . . . . . . . . . . . . . . . . 20

1.2.6. Estimación de $\boldsymbol{v}_{\infty} \ldots \ldots \ldots \ldots \ldots \ldots \ldots$

1.3. Breve reseña histórica del modelado teórico de los vientos estelares. . . 23

1.4. Objetivos de esta tesis . . . . . . . . . . . . . . . 26

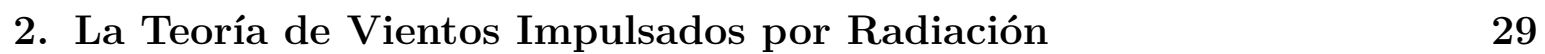

2.1. Idea conceptual . . . . . . . . . . . . . . . . . . . . . . . . . . . . . . . . . 29

2.1.1. Hipótesis de la teoría estándar . . . . . . . . . . . . . . . 30

2.2. Ecuaciones hidrodinámicas básicas . . . . . . . . . . . . . 32

2.2.1. La aceleración debida al continuo de radiación . . . . . . . . 36

2.2.2. La aceleración debida a la absorción de momento en las líneas espectrales. . . . . . . . . . . . . . . . . 37

$2.2 .3 . \quad$ La aproximación de Sobolev . . . . . . . . . . . . . . . 40

2.2.4. Aceleración radiativa para fuentes puntuales . . . . . . . . . . 42

2.2.5. Aceleración radiativa para un conjunto de líneas espectrales . . 44

2.2.6. Incorporación del equilibrio de ionización . . . . . . . . . 45

2.2.7. La función de distribución de intensidades de líneas espectrales. 48

2.2 .8 . Corrección por disco estelar finito . . . . . . . . . . . . . . . . 49

2.2.9. Cálculo de modelos de vientos y parámetros de la aceleración

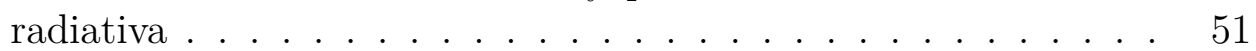

2.2.10. Resolución de las ecuaciones hidrodinámicas en la teoría CAK . 53

2.3. La teoría estándar y los modelos unificados . . . . . . . . . . . . 55

2.4. Tratamientos alternativos de la aceleración de radiación . . . . . . . . . 57

2.5. Relaciones fundamentales de los vientos impulsados por radiación . . . 60

2.5.1. Relación Momento del Viento - Luminosidad (WLR) . . . . . 60 
2.5.2. Relación Gravedad pesada en flujo - Luminosidad

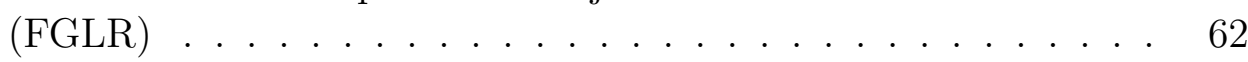

2.6. Apartamientos de los vientos respecto a la teoría estándar . . . . . . . 64

2.6.1. Inestabilidades de los vientos impulsados por radiación . . . . . 64

2.6.2. Mecanismo de bi-estabilidad . . . . . . . . . . . . . . . 66

2.6.3. Discrepancia de masas . . . . . . . . . . . . . . . . 67

2.6.4. La macro-turbulencia y la rotación . . . . . . . . . . . . . . . . 68

2.6.5. Problema de los vientos débiles . . . . . . . . . . . . . . . . . 70

2.6.6. Componentes discretas en absorción. . . . . . . . . . . . . . 71

2.6.7. Discordancias en los parámetros del viento . . . . . . . . . . . . 74

2.6.8. Vientos no homogéneos (clumping) . . . . . . . . . . . 75

3. Vientos en rotación impulsados por radiación $\quad 79$

3.1. Propiedades de los vientos impulsados por radiación en rotación . . . . 79

3.2. Ecuaciones hidrodinámicas para vientos en rotación . . . . . . . . . . . 81

3.2.1. Relaciones de escala para vientos en rotación . . . . . . . . . . . 84

3.3. Soluciones hidrodinámicas para vientos en rotación . . . . . . . . . . . 85

3.3.1. La solución rápida o m-CAK . . . . . . . . . . . . . . . . . . . . 87

3.3.2. La solución $\Omega_{\text {lenta }} \ldots \ldots \ldots$. . . . . . . . . . . . . . . . . . . . . . 89

3.3.3. $\quad$ Aplicaciones de la solución $\Omega_{\text {lenta }} \ldots \ldots$. . . . . . . . . . . . . . 91

3.3.4. La solución $\boldsymbol{\delta}_{\text {lenta }}$. . . . . . . . . . . . . . . . . . . . . . . . . . 93

3.3.5. El código HYDWIND . . . . . . . . . . . . . . . . . . . . . . . 94

4. Dominios de las soluciones de vientos en rotación impulsados por $\begin{array}{ll}\text { radiación } & 97\end{array}$

4.1. Análisis del dominio de las soluciones hidrodinámicas . . . . . . . . . . 97

4.2. "Modelos base" para las estrellas supergigantes B . . . . . . . . . . . . 98

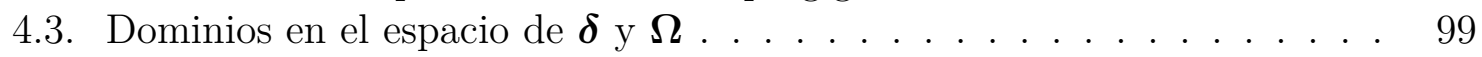

4.3.1. Soluciones para el modelo base T19 . . . . . . . . . . . . . . . . 100

4.3.2. Soluciones para modelos base de temperaturas efectivas bajas e intermedias ................... 107

4.3.3. Soluciones para modelos base de alta temperatura efectiva . . . 108

4.4. Influencia del parámetro $\boldsymbol{k}$ en los dominios de las soluciones . . . . . . 109

4.4.1. La solución lenta en modelos base de alta temperatura efectiva . 111

$4.4 .2 . \quad$ La brecha y el parámetro $\boldsymbol{k} \ldots \ldots \ldots . \ldots . \ldots 111$

4.4.3. La pendiente de la tasa de pérdida de masa y el parámetro $\boldsymbol{k}$. . 112

4.5. Influencia del parámetro $\boldsymbol{\alpha}$ en los dominios . . . . . . . . . . . . . . . . 114

4.5.1. La brecha y el parámetro $\boldsymbol{\alpha}$. . . . . . . . . . . . . . . . 116

4.6. Análisis de la brecha $\ldots \ldots \ldots \ldots$

4.6.1. Soluciones dentro de la brecha . . . . . . . . . . . . . . . . 118

5. El transporte de radiación en medios en movimiento 121

5.1. Transporte de radiación en vientos estelares . . . . . . . . . . . . . . . 121

5.1.1. Transporte de radiación en la aproximación de Sobolev . . . . . 123

5.1.2. Transporte de radiación en la aproximación de Sobolev con con-

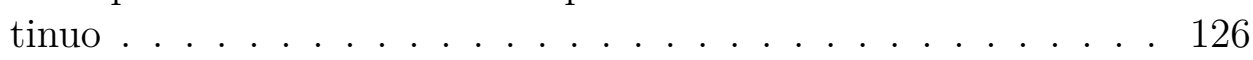

5.1.3. Transporte de radiación en el comoving frame . . . . . . . . . 128 
ÍNDICE GENERAL

5.2. Códigos de cálculo para vientos impulsados por radiación . . . . . . . . 131

5.2 .1 . El código FASTWIND $\ldots \ldots \ldots \ldots \ldots$

5.3. El problema de las inversiones de población . . . . . . . . . . . . . . 134

5.3.1. Inversiones de población en la aproximación de Sobolev con con-

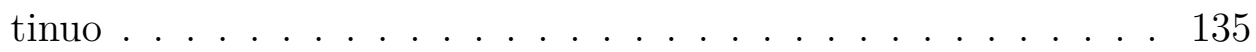

5.3.2. Inversiones de población en el comoving frame . . . . . . . . 137

5.3.3. Implementación en FASTWIND del tratamiento de las inversio-

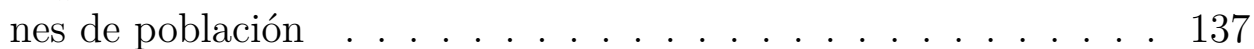

6. Análisis de los perfiles de línea para las diferentes soluciones hidrodinámicas

6.1. Modelado de perfiles de líneas espectrales . . . . . . . . . . . . . . . 141

6.2. Vinculación entre los códigos HYDWIND y FASTWIND . . . . . . . . 143

6.3. Análisis de los perfiles y los parámetros de la fuerza de radiación. . . . . 144

6.3.1. Dependencia de los perfiles de línea con $\boldsymbol{\Omega}$ y $\boldsymbol{\delta}$. . . . . . . . 144

6.3.2. Dependencia de los perfiles de línea con $\boldsymbol{\delta}$ y $\boldsymbol{k}$. . . . . . . 147

6.3.3. Dependencia de los perfiles de línea con $\boldsymbol{\alpha}$. . . . . . . . . 151

6.4. Ajuste de los perfiles sintéticos a observaciones . . . . . . . . . . . 152

6.4.1. Criterios para el ajuste de los perfiles . . . . . . . . . . . 152

6.4.2. Selección de las observaciones . . . . . . . . . . . . . . . . . . 157

$6.4 .3 . \quad$ Ajustes a las observaciones . . . . . . . . . . . . . . . 160

6.4.4. Errores en las estimaciones de los parámetros del viento. . . . . 170

$\begin{array}{ll}\text { 7. Discusión } & 171\end{array}$

7.1. Validez de las aproximaciones de los modelos . . . . . . . . . . . . . 171

7.2. Restricciones a los valores de los parámetros . . . . . . . . . . . . . . 174

7.3. Características físicas de las soluciones lentas . . . . . . . . . . . . . 176

7.3.1. Las soluciones lentas y sus orígenes . . . . . . . . . . . 176

7.3.2. Eficiencia en la transferencia de momento del campo de radiación 177

7.3.3. Parámetros derivados a partir de las mediciones en las estrellas

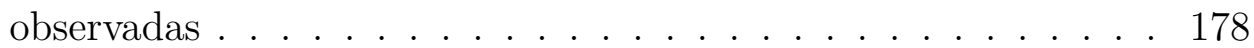

7.3.4. Relación entre $\boldsymbol{v}_{\infty}$ y $\boldsymbol{v}_{\mathrm{esc}} \ldots \ldots \ldots \ldots \ldots$

7.3.5. La relación momento del viento - luminosidad para el régimen

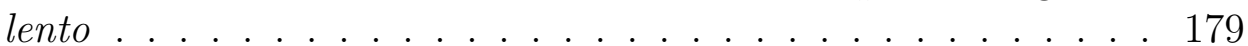

7.3.6. El invariante Q para el régimen lento . . . . . . . . . . . . 181

7.4. Identificación del régimen de viento a partir de los perfiles de línea. . . . 184

7.5. La brecha en los vientos de supergigantes B . . . . . . . . . . . . . 185

7.5.1. Transiciones entre regímenes de viento . . . . . . . . . . . 185

7.5.2. La brecha y la bi-estabilidad . . . . . . . . . . . . . . . 186

7.5.3. Variabilidad del viento en el corto período . . . . . . . . . 187

$\begin{array}{lr}\text { 8. Perspectivas y conclusiones } & 189\end{array}$

8.1. Sobre el viento de las supergigantes B . . . . . . . . . . . . . . . 189

8.2. Sobre las propiedades observadas de las supergigantes B $\ldots . . . . .190$

8.3. Sobre el aporte de las soluciones lenta a la teoría de vientos impulsados por radiación $\ldots \ldots \ldots \ldots \ldots \ldots \ldots \ldots$

8.4. Trabajo a futuro . . . . . . . . . . . . . . . . . 194 
ÍNDICE GENERAL

\begin{tabular}{lr}
\hline Apéndices & 195
\end{tabular} .1. Cálculo de la función $\mathcal{Q}$ para simetría esférica . . . . . . . . . . . . . . 197 


\section{Capítulo 1}

\section{Los Vientos Estelares en las Estrellas Masivas}

Resumen del capítulo: Se presentan los vientos de las estrellas tempranas, sus evidencias observacionales y su relevancia. Se introducen los parámetros usados para caracterizar este fenómeno y se resumen brevemente los modos de estimarlos. Se caracteriza a las estrellas supergigantes $B$, como un subgrupo complejo de las estrellas masivas. Se reseña brevemente el desarrollo histórico de los estudios teóricos de los vientos en las estrellas masivas, especialmente, la teoría de los vientos impulsados por radiación. En el marco general de esta teoría, con la inclusión de los efectos producidos por la rotación, se presentan los objetivos de esta tesis.

\subsection{Los vientos estelares}

Los vientos estelares son flujos de materia expulsados por las estrellas hacia el medio interestelar. En su mayor parte, se componen por protones y electrones, aunque también, en una proporción mucho menor, pueden contener átomos neutros o ionizados de distintos elementos químicos.

Los vientos más intensos son característicos de las estrellas de alta masa con tipos espectrales $\mathrm{O}$, B y A; aunque también son muy notorios en objetos que transitan algunas etapas evolutivas particulares tales como las estrellas Wolf-Rayet (WR), las variables luminosas azules (LBV) o las estrellas centrales de nebulosas planetarias (CSPN).

Los vientos estelares son detectados, de manera directa, a través de sus manifestaciones en el espectro continuo y en las líneas espectrales, en las estrellas que superan ciertos límites en luminosidad dentro del diagrama de Hertzprung-Russell (diagrama HR). Particularmente, todas las estrellas de tipos espectrales O, B y A con luminosidades que superan el valor de $10^{4} L_{\odot}$ (donde el símbolo $\odot$ se refiere al Sol) presentan claros indicios de vientos estelares durante todas sus etapas evolutivas (ver por ejemplo, Abbott 1979). En el caso de las estrellas de masa intermedia o baja $\left(M_{Z A M S}<8 M_{\odot}\right.$, siendo $M_{Z A M S}$ la masa de la estrella al iniciar su etapa en la secuencia principal o Zero Age Main Sequence mass), esto ocurre cuando sus luminosidades superan el valor de $10^{3.6} L_{\odot}$, lo que corresponde a estrellas en la fase post-AGB (etapas posteriores a la rama asintótica de las gigantes), y desplazándose hacia la secuencia de las enanas blancas (Pauldrach et al. 1988). 


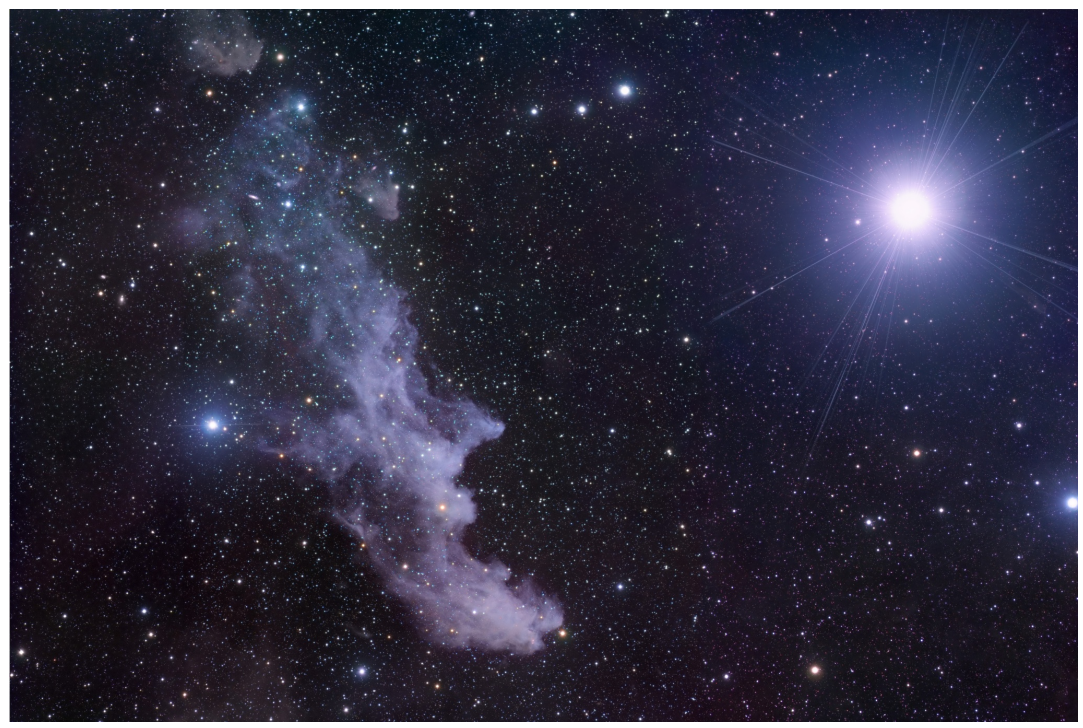

Figura 1.1. La estrella supergigante azul tardía Rigel (B8 Ia), situada a 260 pc, con su flujo extraordinario de energía ilumina la nebulosa de reflexión Witch Head (IC 2118). Imagen tomada en el Star Shadows Remote Observatory.

La clara vinculación entre la luminosidad de la estrella y la presencia de vientos estelares intensos en su entorno, pone en evidencia que el principal responsable de la impulsión del viento es el campo de radiación estelar. A partir de la hipótesis que propone que la presión generada por el campo de radiación de una estrella temprana es capaz de poner en movimiento el plasma de su atmósfera (Milne 1926), se ha desarrollado la teoría estándar de vientos impulsados por radiación.

Esta tesis se inserta en el marco conceptual de esta teoría, por lo que sus ideas básicas son examinada en detalle en los capítulos siguientes. Previamente, se presentan algunas características propias de los vientos en las estrellas masivas, especialmente de las supergigantes de tipo espectral B, que son el objeto de estudio de este trabajo.

\subsubsection{Evidencias de los vientos estelares}

Los vientos estelares se manifiestan a través de numerosas características que pueden observarse en los espectros estelares y en imagen directa. El modelado teórico y semiempírico de estos rasgos permite estimar las condiciones físicas globales de los vientos e inferir información acerca de los mecanismos que los generan. Entre las evidencias más notorias que demuestran la presencia de vientos estelares se pueden citar:

\section{- Perfiles de tipo P Cygni}

El indicador más claro de la presencia de un viento estelar es el perfil de línea de tipo P Cygni, como los que se muestran en la figura 1.2(a) en el rango espectral del UV. Los perfiles P Cygni tienen dos componentes, una en emisión, aproximadamente centrada respecto a la longitud de onda de reposo, y otra en absorción, desplazada hacia el azul. La figura 1.2(b) muestra la formación de esta clase de perfil. La componente en absorción se forma por la disminución del flujo 
de fotones provenientes de toda la superficie estelar con dirección al observador, los cuales han sido dispersados (scattered) en otras direcciones. Como se forma al frente de la envoltura en expansión, entre el observador y la estrella, el desplazamiento Doppler ubica a esta componente del perfil en longitudes de onda más cortas (región F de la figura 1.2(b)]. Por su parte, la componente en emisión está formada por la contribución de fotones dispersados o generados por recombinación tanto enfrente del disco como en el halo, la extensa región que rodea a toda la estrella (región H). Como el material en el halo está en expansión, los fotones generados en esta zona saldrán de iones con velocidades radiales tanto positivas como negativas, resultando en una ancha emisión sin desplazamiento Doppler neto. En realidad, hay una pequeña asimetría en esta componente debido a la ausencia de contribución a la línea, proveniente de la parte del viento bloqueada por el disco estelar (en la dirección opuesta al observador, región O).

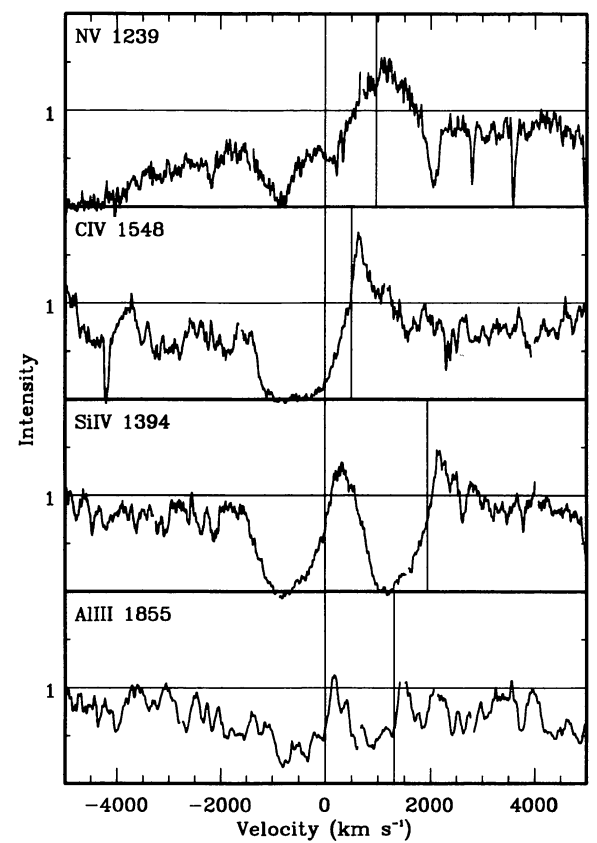

(a)
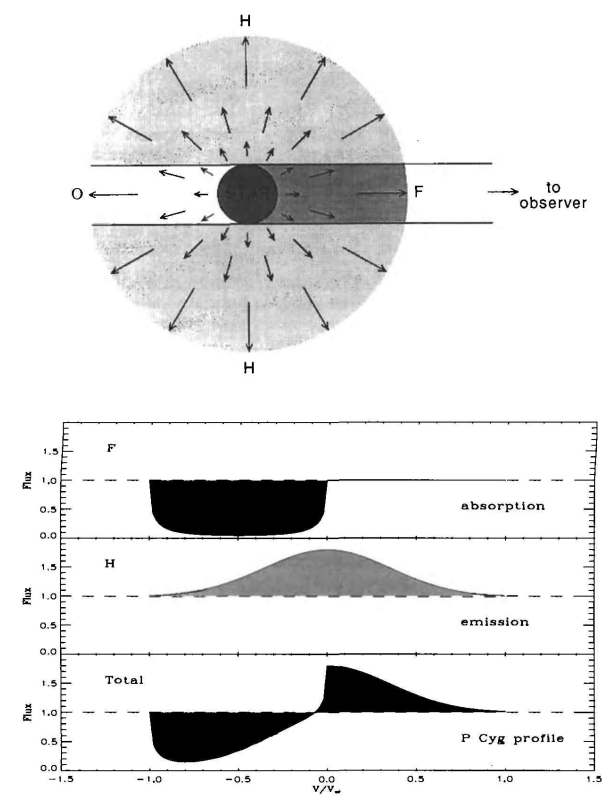

(b)

Figura 1.2. a) Perfiles de tipo P Cygni en las líneas de N V, C IV, Si IV y Al III, observados en el UV por el satélite IUE, en el espectro de la estrella HD 52382 (B0.5 Ia), dando evidencia de un intenso viento estelar. En cada caso, puede observarse un doblete de líneas de cada elemento, con distinta ubicación en velocidad marcada por las líneas verticales. En el caso de la línea de C IV, los perfiles se encuentran superpuestos debido a la escasa separación entre las mismos. A su vez, para cada línea de los dobletes, se distinguen las dos componentes del perfil P Cygni: una en emisión, generalmente centrada en la longitud de onda de reposo de la línea, y otra en absorción, desplazada hacia el azul. Figura tomada de Snow et al. (1994). b) Formación de un perfil de tipo P Cygni a partir de un viento, con la contribución de cada región al perfil. Figura tomada de Lamers \& Cassinelli (1999).

Las primeras observaciones en el UV de esta clase de perfil de línea (Morton 
1967a b; Snow \& Morton 1976) fueron la evidencia definitiva de que el fenómeno de los vientos estelares era ubicuo en las estrellas tempranas.

Las líneas con perfiles P Cygni pueden aparecer en cualquier región espectral pero, para las estrellas tempranas con vientos intensos, predominan en la región UV. Se trata de líneas de resonancia (ya que involucran el nivel fundamental y el primer nivel excitado de los iones que las producen) de elementos altamente ionizados, y se forman cuando un ión que ha absorbido un fotón, lo reemite inmediatamente de manera espontánea en cualquier dirección. Los perfiles pueden ser muy intensos, dependiendo de la intensidad de oscilador de la línea y de la densidad de columna de los iones. Para las estrellas de tipo O y B tempranas, son características las líneas con perfiles P Cygni de C IV, N V, Fe II y Si IV. Para las estrellas B tardías y A, aparecen con estos perfiles las líneas de C II, mientras que, para las estrellas más frías, se dan en líneas de Mg II. Los altos niveles de ionización en los átomos que forman estas líneas constituyen el fenómeno de super-ionización (Lamers \& Snow 1978), un estado de alta ionización que no es esperado para las temperaturas efectivas de las estrellas. Pauldrach (1987) pudo modelar este fenómeno en estrellas $\mathrm{O}$, por medio de la resolución detallada del equilibrio de ionización y las ecuaciones de equilibrio estadístico. Sin embargo, en muchos casos, este fenómeno ha sido atribuido principalmente a la presencia de flujos de rayos X (de Koter et al. 2000, Lamers 2013).

\section{- Líneas espectrales en emisión o absorción}

La mayoría de las líneas en emisión de los vientos son producidas por recombinación en niveles atómicos altos seguida de desexcitación. Los perfiles en emisión de la línea $\mathrm{H} \alpha$ del hidrógeno $(\lambda 6562 \AA$ ) son típicos en las estrellas de tipo O y B supergigantes, como así también las líneas $\mathrm{P} \alpha$ y $\operatorname{Br} \alpha$ (figura 1.3), en la región del infrarrojo cercano (near infra-red, NIR) (Najarro et al.|2011). Del mismo modo, algunas líneas de helio ionizado también pueden presentar emisión. En general, las líneas en emisión muestran perfiles simétricos, centrados en la longitud de onda de reposo y su intensidad depende fuertemente de la densidad del viento, ya que son formadas por recombinación.

Por otro lado, las líneas en absorción formadas en el viento son producidas por la dispersión o scattering de los fotones del campo de radiación estelar, en direcciones diferentes a las del observador. Estas líneas son más anchas que las originadas en la fotosfera estelar, debido al efecto Doppler que se produce sobre los fotones por el movimiento del material. Esta clase de líneas son muy comunes en la región UV del espectro (figura 1.4).

\section{- Excesos en el continuo de radiación}

La contribución más notoria del viento al espectro continuo estelar son los excesos de flujo infrarrojo (IR), submilimétrico y de radio, que excede al flujo fotosférico predicho por la teoría de atmósferas estelares estáticas (figura 1.5). La mayor parte de los excesos se origina en la radiación térmica creada a partir de transiciones libre-libre o ligado-libre en el viento. Las opacidades correspondientes a estos procesos tienen una dependencia cúbica con la longitud de onda, $\lambda^{3}$, por lo que son más importantes en las longitudes de onda más largas. También puede 


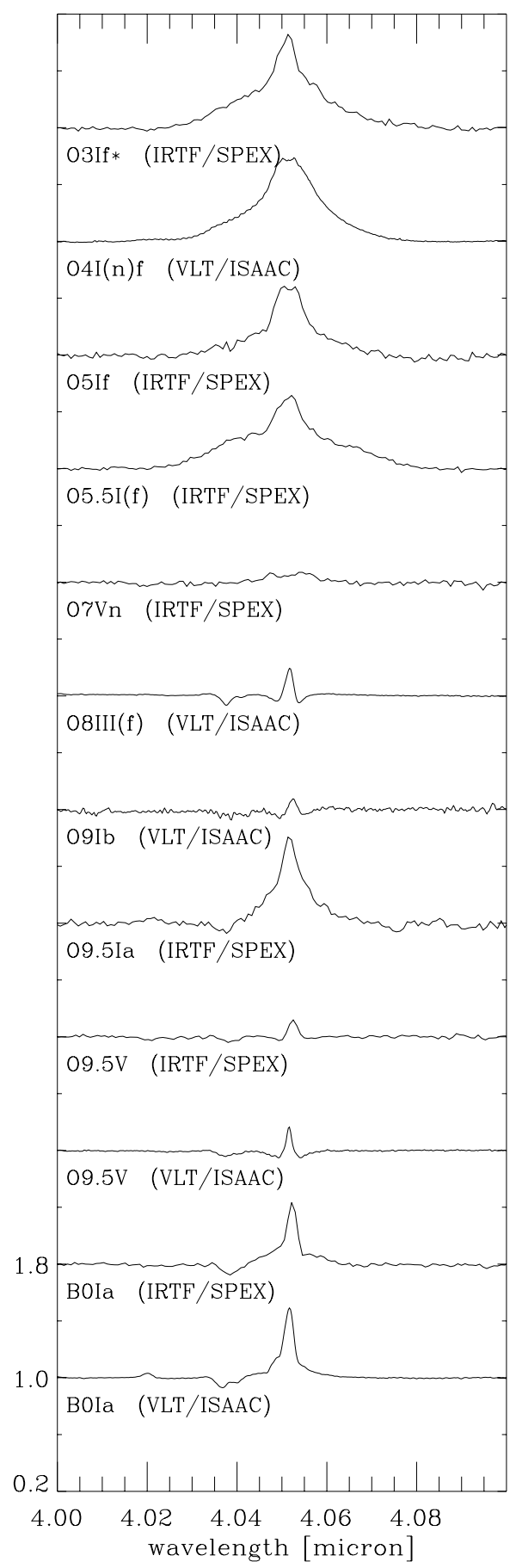

Figura 1.3. Ejemplos de perfiles en emisión de la línea $\operatorname{Br} \alpha$ (4051 nm en la banda L del IR) en estrellas tempranas, tomados con los instrumentos indicados entre paréntesis. Las líneas en emisión en el IR son claros indicios de los vientos estelares. Figura tomada de Najarro et al. (2011).

haber una contribución de radiación no térmica pero, en el caso de los vientos, su contribución no supera el $30 \%$ de la emisión total (Bieging et al. 1989). Como 


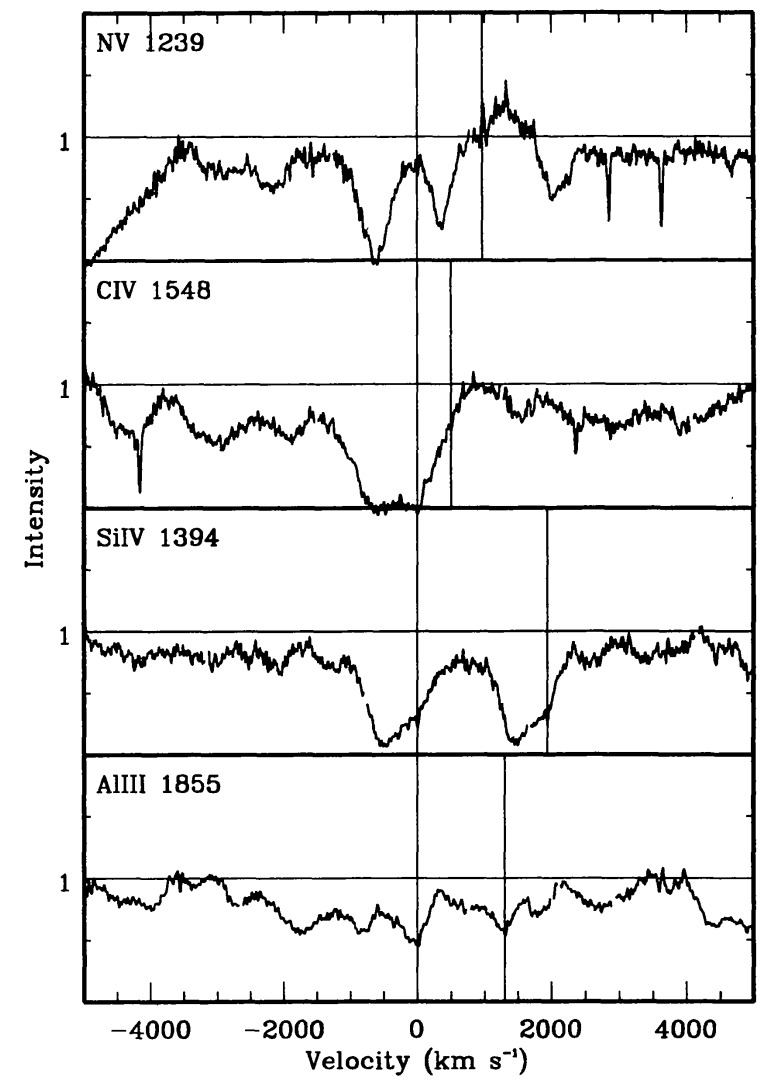

Figura 1.4. Perfiles de absorción en el UV para la estrella HD 17246 o $\gamma$ Ara (B1.5 Ib). Claramente los perfiles no tienen forma gaussiana, indicando la presencia del viento estelar. Figura tomada de Snow et al. (1994).

las estrellas OB son fuentes de radio débiles, los estudios de sus vientos mediante observaciones en ese rango de frecuencias, se limitan a estrellas de nuestra galaxia y con vientos densos (ver, por ejemplo, Runacres \& Blomme 1996; Benaglia et al. 2007).

Todas las estrellas O son intensos emisores de rayos X blandos (ver, por ejemplo, el catálogo de Chlebowski et al. 1989) y su luminosidad en rayos X está correlacionada con la luminosidad bolométrica. El origen de los rayos X puede deberse a ondas de choque que se propagan en el viento, originadas por las inestabilidades inherentes a los vientos impulsados por radiación (ver sección 2.6.1) o por colisiones de concentraciones locales de densidad o grumos (clumps, ver sección 2.6.8. También se considera el caso de estrellas binarias con una compañera compacta, la cual irradia al viento cambiando su equilibrio de ionizaciones (Krtička \& Kubát 2016). Las estrellas $\mathrm{B}$ tempranas también presentan emisión de rayos $\mathrm{X}$ blandos pero, sus vientos son ópticamente delgados a esta radiación, a diferencia de las estrellas O, cuyos vientos son ópticamente gruesos (Kudritzki \& Puls 2000).

Algunas estrellas B tempranas de clases de luminosidad entre V y II presentan un intenso exceso en la región del ultravioleta extremo (EUV). Uno de los casos más notorios es la estrella gigante $\epsilon$ CMa (B2 II), que fue detectada por los satélites 


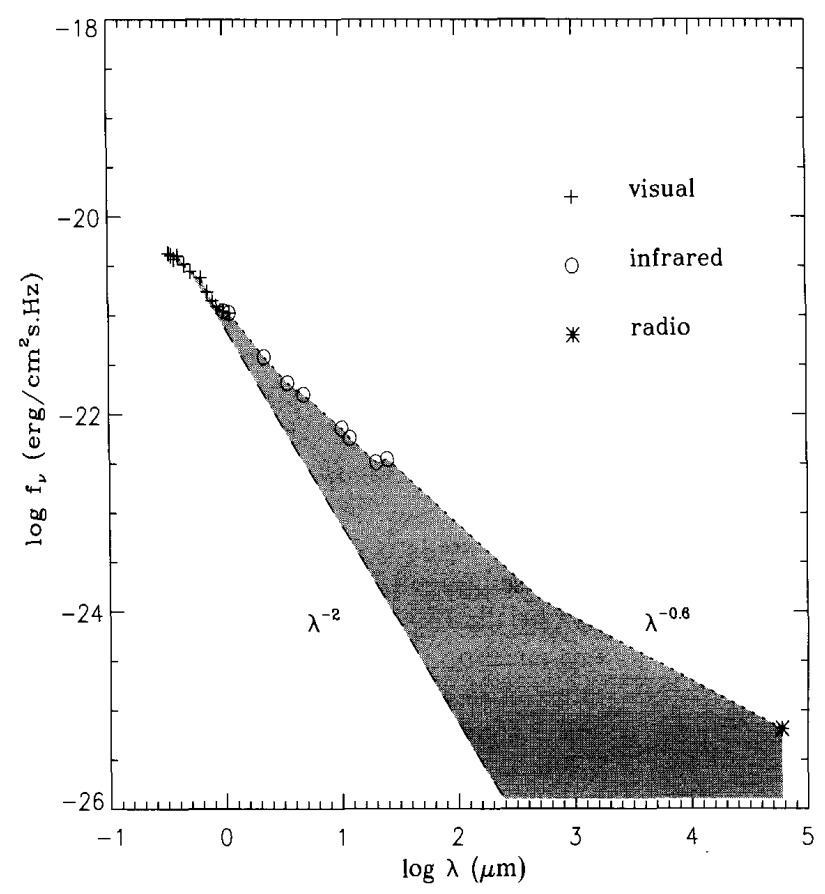

Figura 1.5. Distribución espectral de energía de la estrella LBV P Cygni (B1-2 Ia-0ep). La línea a rayas indica el espectro continuo esperado para una fotosfera en equilibrio hidrostático (sin viento). El área sombreada corresponde a los excesos de energía en longitudes de onda largas, indicando claramente la presencia del viento. Figura tomada de Lamers \& Cassinelli (1999).

EUVE y ROSAT como una de las fuentes EUV más brillantes del cielo (Hoare et al. 1993; Cassinelli 1994), en el continuo de Lyman, entre 500 y 700 Å. Según el trabajo de Cassinelli, su flujo en el continuo de Lyman sería unas 30 veces mayor que lo previsto por los modelos de atmósfera que incorporan line blanketing (ver sección 2.3 y la discrepancia sería aún mayor a ambos lados del salto de 504 A del He I. Estas características se atribuyen a la presencia de viento y están vinculadas a la producción de rayos X y líneas de emisión en el EUV. Najarro et al. (1996) analizaron la influencia del viento en la formación de los continuos del $\mathrm{H}$ y $\mathrm{He}$ en estrellas $\mathrm{B}$ y encontraron una fuerte correlación entre la pérdida de masa y el número de fotones ionizantes de $\mathrm{H}$ y He.

\subsubsection{Relevancia de los vientos estelares}

El fenómeno de los vientos estelares tiene una gran influencia en numerosos objetos y ámbitos astrofísicos. Los cálculos evolutivos de estrellas masivas deben incluir la presencia de vientos estelares ya que, a través de ellos, las estrellas pueden perder una parte significativa de sus masas iniciales, lo cual altera considerablemente su evolución posterior (ver, por ejemplo, Meynet et al. 1994, Ekström et al. 2012; Lamers 2013; Vink 2014). Debido a la presencia de estos vientos, también resultan modificadas las propias luminosidades, las abundancias superficiales, los perfiles químicos (estratificación 
hacia el interior de la cantidad de las especies atómicas), los tiempos y las secuencias evolutivas y las clases de objetos finales (estrella de neutrones o agujero negro). En las estrellas WR, los vientos producen la liberación de las capas externas, exponiendo una composición superficial completamente diferente.

A partir de los vientos estelares, el medio interestelar (interstellar medium, ISM) resulta enriquecido con los elementos elaborados en los interiores estelares. Si bien la forma más eficiente de enriquecimiento se produce durante las explosiones de supernova, los elementos químicos creados en los interiores estelares que hayan alcanzado la superficie de la estrella por algún mecanismo (por ejemplo, la convección o la difusión), pueden ser expulsados como parte del viento estelar. De esta manera, los vientos estelares realizan una lenta y permanente contribución de elementos pesados al ISM.

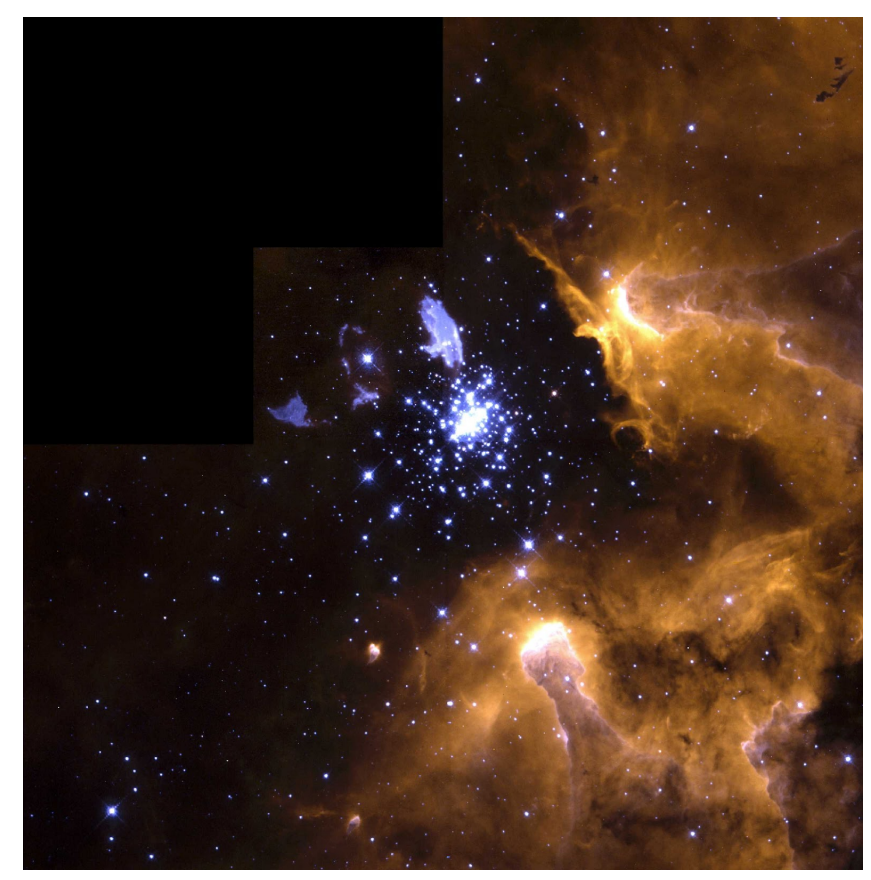

Figura 1.6. La nebulosa NGC 3603 es una gigantesca región HII en el brazo espiral de Carina, cuyo gas es ionizado por las estrellas del cúmulo abierto cercano HD 97950. En la fotografía destaca la estrella supergigante azul Sher 25 (B1 Iab), ubicada arriba y a la izquierda de la imagen, que exhibe un anillo de gas azul brillante (semejante al anillo observado actualmente en el remanente de la supernova 1987A) y filamentos de flujo bipolar. Imagen tomada por el Hubble Space Telescope.

A través de la absorción de la radiación, la presencia de vientos modifica sensiblemente la radiación ionizante de las estrellas disponible en el medio para producir regiones H II. La dinámica y el balance energético del ISM reciben una importante contribución de momento y energía de los vientos (figura 1.6), tal como se muestra, por ejemplo, en Leitherer et al. (1992). Todos estos aportes conducen a redistribuir la materia, creando burbujas, frentes de choque y superburbujas alrededor de las estrellas masivas, contribuyendo a la formación estelar y estableciendo las condiciones físicas mismas del medio interestelar dentro de cúmulos, nebulosas y las galaxias en su totalidad (Freyer et al. 2003). La interacción de vientos de distinta intensidad en diferentes 
etapas evolutivas de las estrellas puede generar estructuras complejas de material que pueden observarse, por ejemplo, en nebulosas planetarias. La energía y momento inyectado al medio interestelar por el viento a lo largo de toda la evolución estelar, es comparable al de una supernova (Abbott 1982a; Lamers 2001).

Además de momento y energía, los vientos también se llevan momento angular de sus estrellas en rotación. Esto tiene importancia, por ejemplo, en la formación de los "Estallidos de Rayos Gamma" (Gamma Ray Bursts, GRBs) a partir de estrellas WolfRayet de baja metalicidad. En esos casos, la pérdida de momento angular a través del viento, puede reducir la velocidad angular del núcleo de la estrella, inhibiendo el mecanismo que lleva a detonarlos, a menos que el núcleo se encuentre desacoplado de la envoltura (Lamers 2013).

Sin embargo, el aporte más importante del análisis espectroscópico de los vientos de supergigantes azules de la Vía Láctea o de galaxias cercanas, es la estimación de sus distancias a partir de la relación Momento del viento-Luminosidad (Wind momentumLuminosity Relationship, WLR; Kudritzki et al. 1995, Puls et al. 1996, Kudritzki et al. 1999). Incluso en galaxias lejanas y hasta en objetos que presentan lentes gravitacionales, se puede obtener importante información tal como las abundancias químicas en regiones de formación estelar o la distribución de las poblaciones estelares y de sus metalicidades, a través del estudio de los espectros integrados de los vientos de sus poblaciones estelares.

Más allá de los efectos que los vientos estelares puedan tener sobre su entorno o sobre los sistemas astrofísicos que contienen a las estrellas masivas que los generan, estos extraordinarios flujos de materia son fenómenos de mucho interés en sí mismos. Estudiar los mecanismos de impulsión junto a las distribuciones de densidad y las estructuras de velocidad que desarrollan a lo largo de su extensión, tiene gran relevancia ya que los efectos de este fenómeno son observables directamente en el espectro de las estrellas; y que, junto con el flujo de energía radiativa de las estrellas, son las manifestaciones propias de estos sistemas físicos.

\subsection{Las estrellas supergigantes B}

El dominio de estrellas supergigantes B es, sin dudas, uno de los espacios más ricos entre las estrellas masivas para la exploración de nuevos modelos hidrodinámicos de vientos estelares. Son el subgrupo más numeroso de estrellas masivas luminosas (Searle et al. 2008). Sus masas abarcan un amplio rango, comprendido aproximadamente entre 8 y $50 \mathrm{M}_{\odot}$ y sus luminosidades, con valores entre $10^{5}$ y $10^{5.6} L_{\odot}$.

La teoría estándar de vientos impulsados por radiación describe muy adecuadamente el viento de las estrellas O. Sin embargo, exceptuando a las supergigantes B más tempranas, las que se equiparan en estas características a las $\mathrm{O}$, los ajustes de la teoría de vientos a las supergigantes B intermedias o tardías son imprecisos y están sujetos a considerables discrepancias (sección 2.6). De acuerdo a Markova \& Puls (2008) eso se debe, en gran parte, a que la temperatura efectiva más baja produce una mayor población de especies atómicas que ejercen influencia en los mecanismos de impulsión del viento, modificando las condiciones físicas del material y volviéndolo más dificil de modelar. 


\subsubsection{Grupos de supergigantes B}

Las estrellas supergigantes B conforman una mezcla bastante heterogénea de objetos en estados evolutivos diferentes, los que son transitados en tiempos astronómicos muy cortos. Esta situación produce pocos miembros en cada estadio evolutivo, haciendo muy difícil un estudio estadístico destinado a determinar y ensamblar cada etapa en una simple secuencia evolutiva. También, las secuencias evolutivas pueden depender de condiciones iniciales adicionales de la estrella, además de la masa, tales como la metalicidad o la rotación. Por estos motivos puede ocurrir que algunos objetos pertenecezcan a diferentes grupos simultáneamente, presentando características comunes y dificultando su clasificación.

Otra de las características del dominio de las supergigantes B es la variabilidad fotométrica y espectroscópica que parece estar presente, con diferentes manifestaciones, en la mayoría de los objetos. El origen y las propiedades de la variabilidad son materia de extensa investigación y debate (por ejemplo, Lefever et al. 2007, Chesneau et al. 2011; Puls et al. 2011; Kraus et al. 2014).

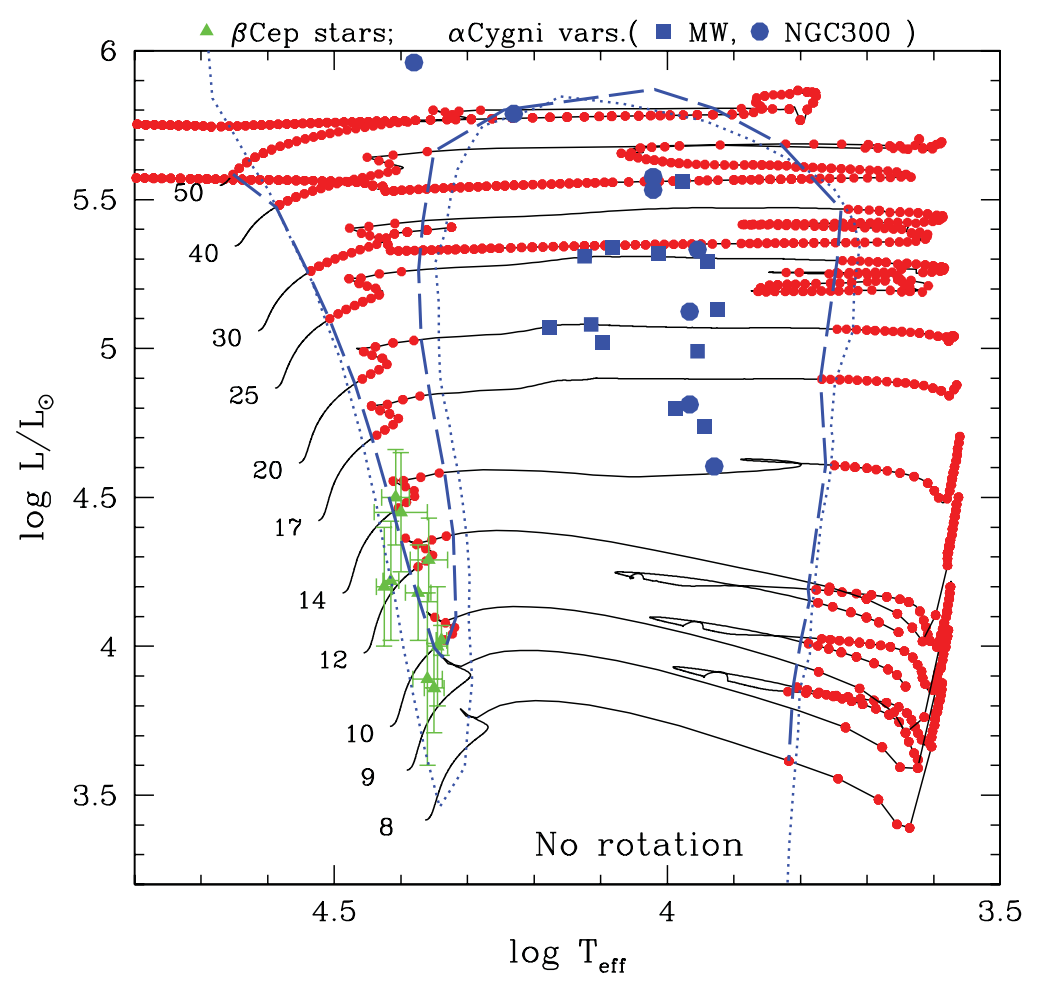

Figura 1.7. Caminos o tracks evolutivos para las estrellas masivas, calculados sin rotación por Saio et al. (2013). Los puntos rojos indican los lugares en los cuales resulta excitado al menos un modo radial de pulsación. Los círculos y cuadrados azules indican la posición de las variables $\alpha$ Cygni, mientras que los triángulos verdes indican la localización de las variables $\beta$ Cephei. En la figura también puede observarse el lazo que involucra un doble cruce evolutivo por la región ocupada por las supergigantes B, en los casos de estrellas con masas iniciales mayores a $20 \mathrm{M}_{\odot}$. Figura tomada de Saio et al. (2013). 
A continuación se listan algunos grupos de objetos con propiedades comunes, en los cuales aparecen las supergigantes B. La mayoría de estos grupos presenta variabilidad intrínseca; por lo que se mencionan sus principales características y la diversidad de cambios que experimentan. Es importante señalar que esta síntesis tiene interés meramente ilustrativo, y que no se pretende lograr una descripción completa de estos grupos tan complejos.

a) Estrellas de tipo $\boldsymbol{\alpha}$ Cygni: Conforman un extenso grupo de estrellas supergigantes B y A de la Vía Láctea o de las Nubes de Magallanes con $L_{*} / L_{\odot} \gtrsim 4.6$, que muestran microvariabilidad en luminosidad y en velocidad radial van Leeuwen et al. 1998; Bresolin et al. 2004). Las amplitudes en la curva de luz son del orden de 0.2 magnitudes. Las escalas de tiempo de estas microvariaciones son del orden de días a meses. Las microvariaciones se interpretan como originadas por actividad pulsacional (Sterken 2003). La ubicación de algunas estrellas $\alpha$ Cygni en el diagrama HR puede verse en la figura 1.7.

b) Estrellas SPB (slowly pulsating B stars): Waelkens (1991) adoptó esa denominación para distinguir un grupo de estrellas B intermedias variables, con tipos espectrales ubicados entre B3 y B9, para las cuales detectó variaciones en brillo y color multiperiódicas, en datos fotométricos. Los períodos comprenden el intervalo entre 0.8 y 3 días. Las variaciones en los perfiles de líneas también son conocidas y estudiadas desde hace mucho tiempo. Se considera que estas estrellas tienen actividad pulsacional en términos de modos g (Aerts et al. 2010). Las masas de las estrellas SPB están comprendidas entre 2 y $7 \mathrm{M}_{\odot}$ y todas las detectadas son rotadores lentos (De Cat 2002). La ubicación en el diagrama HR puede verse en la figura 1.8, donde queda claro que la mayoría pertenece a la secuencia principal. Sin embargo, también hay SPB gigantes y supergigantes.

c) Variables S Doradus: Son llamadas también LBVs (Luminous Blue Variable) y se trata de estrellas calientes, masivas y evolucionadas que muestran distintas clases de variabilidad fotométrica intrínseca. Estos objetos exhiben cuatro formas diferentes de variabilidad; algunas de las cuales pueden darse en simultáneo. Estas son: microvariaciones, fases S Dor, variabilidad estocástica y erupciones.

Las microvariaciones son cambios en la magnitud menores o iguales a 0.1 magnitudes (Humphreys \& Davidson 1994). Estas microvariaciones pueden darse también en otras estrellas luminosas azules (por ejemplo, las microvariaciones son propias del grupo de las $\alpha$ Cygni), pero no todas las estrellas que presenten microvariaciones son variables de tipo S Dor ya que, para serlo deben presentar también las demás clases de variabilidad (van Genderen 2001).

Las fases S Dor son fases cíclicas de abrillantamiento, en escalas temporales de años o décadas, y amplitudes de hasta 2.5 magnitudes van Genderen 2001; Sterken 2003).

Las variaciones estocásticas son cambios irregulares que exceden los errores observacionales, algunos de los cuales pueden deberse a eventos cataclísmicos. Su causa probablemente esté relacionada con fenómenos de súbita pérdida o transferencia de masa en el caso de sistemas binarios. 


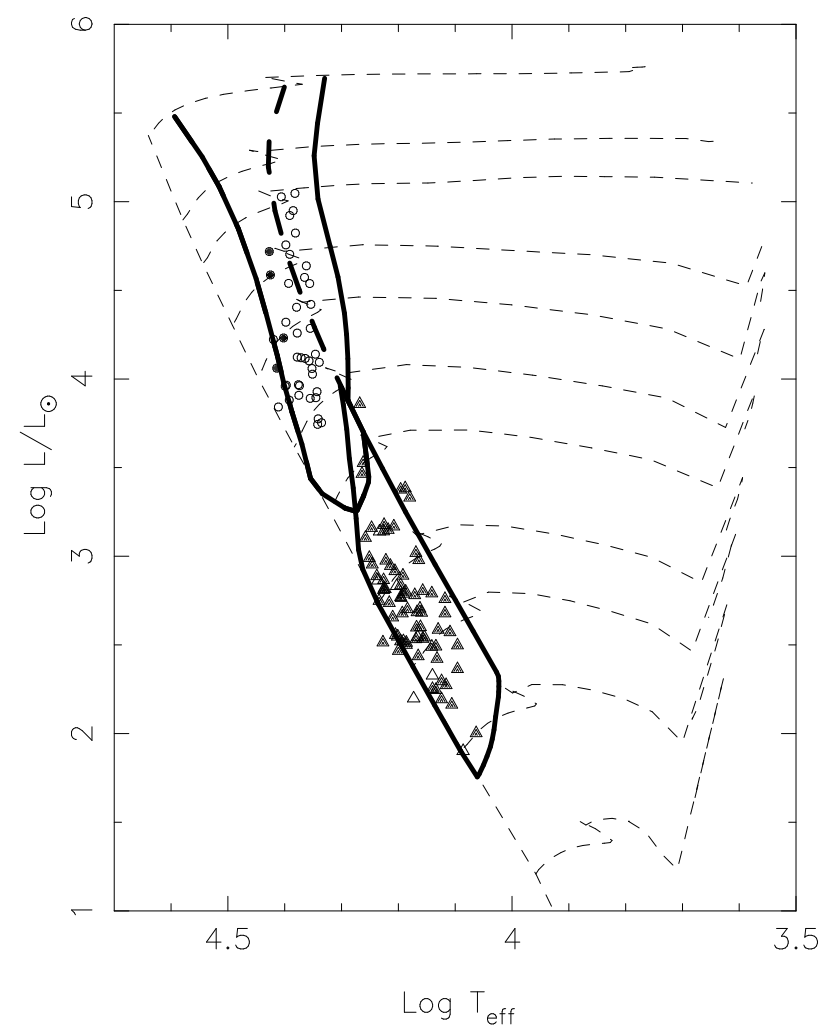

Figura 1.8. Ubicación de las estrellas $\beta$ Cephei (círculos llenos y vacíos) y de las SPB (triángulos llenos y vacíos). Los bordes de las bandas de inestabilidad están indicadas con líneas llenas, mientras que la línea gruesa a rayas corresponden a la TAMS (terminal age main sequence) o límite de la secuencia principal donde termina la fusión de $\mathrm{H}$ en el núcleo estelar. Las líneas delgadas a rayas son los caminos evolutivos. Figura tomada de Waelkens et al. (1998).

También son notorios los infrecuentes pero descollantes eventos eruptivos, por los cuales son muy conocidas las dos variables S Dor más destacadas: $\eta$ Carinae y $\mathrm{P}$ Cygni.

d) $\boldsymbol{\beta}$-Cephei: Se trata de un grupo de estrellas jóvenes pulsantes de Población I próximo a la secuencia principal. Sus masas están comprendidas entre 8 y 18 $\mathrm{M}_{\odot}$, y que oscilan con modos $\mathrm{p}$ y g, con períodos comprendidos entre 2 y 8 horas (Aerts et al. 2010). Se conocen más de 100 miembros cuya amplia mayoría está compuesta por enanas y gigantes. Si bien no se han encontrado oscilaciones semejantes a las correspondientes a estrellas $\beta$ Cephei en estrellas más luminosas que $\log \mathrm{L} / \mathrm{L}_{\odot}>5$ y $\mathrm{M}>20 \mathrm{M}_{\odot}$, no se descarta encontrarlas ya que la teoría (Pamyatnykh 1999) predice que la franja de inestabilidad se extiende desde la secuencia principal hacia estrellas más evolucionadas (figura 1.8). Los objetos de este grupo muestran variabilidad espectroscópica y fotométrica multiperiódica, con variaciones casi sinusoidales en velocidad y curvas de luz. Hay una pequeña superposición entre las franjas de inestabilidad teóricas de estas estrellas con las 
SPB, aunque no se conocen objetos híbridos.

e) Estrellas supergigantes $\mathbf{B}[\mathbf{e}]$ : Las $\operatorname{sgB}[\mathrm{e}]$ son un subgrupo homogéneo de las supergigantes B o B luminosas $\left(\log L_{*} / L_{\odot}>4.0\right)$ con el fenómeno B[e] (Lamers et al. 1998). De acuerdo a Miroshnichenko (2006), el fenómeno B[e] (propio de las estrellas $\mathrm{B}[\mathrm{e}]$ o Be peculiares) se manifiesta a través de la presencia de líneas de Balmer, Fe II y líneas de baja excitación en emisión, junto con excesos en el cercano e intermedio IR y líneas prohibidas en emisión de [O I], [Fe II], [N II] y, en ocasiones, [O III]. Los perfiles de las líneas de Balmer pueden ser de tipo P Cygni con anchos equivalentes mayores a $100 \AA$ para la línea $\mathrm{H} \alpha$. Los excesos IR indican la presencia de polvo caliente circunestelar, con temperaturas de $1000 \mathrm{~K}$ (Zickgraf et al. 1986). Estas manifestaciones del fenómeno B [e] se presentan en grupos muy variados entre los que se encuentran objetos tales como estrellas simbióticas, binarias cerradas, objetos de pre-secuencia principal o nebulosas planetarias. Se supone que poseen un anillo o cáscara circumestelar con formación de polvo (Bjorkman 1998).

\subsubsection{Estado evolutivo}

La evolución de las estrellas masivas es muy compleja y fuente de permanente discusión. De hecho, hay debates básicos sobre el estado evolutivo de las supergigantes B; incluyendo la incerteza de si algunas de ellas se encuentran aún en la etapa de quema de $\mathrm{H}$ de la secuencia principal o si ya están quemando He en etapas posteriores (Vink et al. 2010; Georgy et al. 2013).

De acuerdo a los modelos con rotación de Ekström et al. (2012), una estrella con masa superior a $20 \mathrm{M}_{\odot}$ realiza camino evolutivo que involucra un lazo por el que transita la estrella, pasando de la zona azul del diagrama HR a la zona roja, para volver a la zona azul (ver figura 1.7).

De este modo, una estrella masiva puede transformarse en una estrella supergigante azul proveniendo de dos regiones: directamente de la secuencia principal del diagrama HR, o evolucionando desde la etapa de supergigante roja. Podría esperarse que las estrellas del segundo grupo tengan sus superficies contaminadas por materia procesada en el ciclo CNO y envolturas más reducidas por sus vientos en la etapa de supergigante roja. De acuerdo a Saio et al. (2013), la proporción de supergigantes azules provenientes de una u otra etapa previa, depende de factores poco conocidos tales como la mezcla interna o la pérdida de masa acontecida en la etapa de las supergigantes rojas. En principio, sería posible distinguir entre las estrellas de ambos grupos, si se pudieran determinar las abundancias superficiales de los elementos del ciclo CNO, investigación que está siendo llevada a cabo por muchos investigadores.

Sin embargo, una de las dificultades que acarrea la medición de las abundancias de los elementos del CNO, es que hay una gran dispersión en las velocidades de rotación para estos objetos lo que, de acuerdo a los modelos, produciría diferentes grados de mezclas internas durante la secuencia principal. También, las mediciones de abundancias se encuentran afectadas por la presencia de binarias cercanas o de campos magnéticos, si los hubiere.

Como vía alternativa para resolver el problema de la procedencia de las supergigantes azules, evitando las dificultades mencionadas, Saio et al. (2013) propusieron 
analizar los modos (radiales y no radiales) de pulsación que presentarían estos objetos en uno u otro caso. Estos autores encuentran que los modos radiales son excitados en la región de las supergigantes azules sólo en los modelos en los cuales estas estrellas han estado, previamente, en la etapa de las supergigantes rojas. Además, para el caso de una etapa posterior a la de supergigante roja, los análisis conducen a que se excitarían muchos más modos no radiales de pulsación que en el otro caso. De esta manera, Saio et al. (2013) sugieren una forma de reconocer la procedencia de las supergigantes azules, por medio del estudio de las pulsaciones.

Algunos trabajos han comenzado a probar esta propuesta, como es el caso de (Kraus, Nickeler, Haucke, Cidale, Venero, Fernandes, Tomić, \& Curé 2014). En ese trabajo, que forma parte de la Tesis Doctoral, en realización, del Lic. M. Haucke, y del cual el autor de la presente tesis es co-autor, se estudia detalladamente a 55 Cyg (HD 198 478), una estrella de clase espectral B3 Ia que presenta una variabilidad espectral y fotométrica con múltiples períodos. Combinando el análisis de los modos de pulsación derivados, las fases de pérdida de masa variables a través del viento y la presencia de materiales provenientes del ciclo CNO, se propone que muy probablemente se trate de un objeto en una fase posterior a la de supergigante roja, cruzando el diagrama HR hacia la región azul.

A modo de resumen de las complejas secuencias evolutivas, Maeder (2009) cita numerosos escenarios para las conexiones entre las diferentes clases de estrellas masivas (cuyos acrónimos pueden consultarse en la tabla 1.1) y en los que se puede ver la posible doble procedencia de las supergigantes azules.

A continuación se presentan los escenarios propuestos por Maeder (2009), los que pueden englobarse en tres grupos principales según sus masas. En las secuencias correspondientes, se resaltan las etapas de supergigantes azules (de tipos espectrales $\mathrm{O}$ y B), y se las indica en negrita como BSG, ya que son de interés para este trabajo. Los paréntesis indican etapas evolutivas muy breves y las flechas dobles, posibles idas y vueltas entre dos etapas.

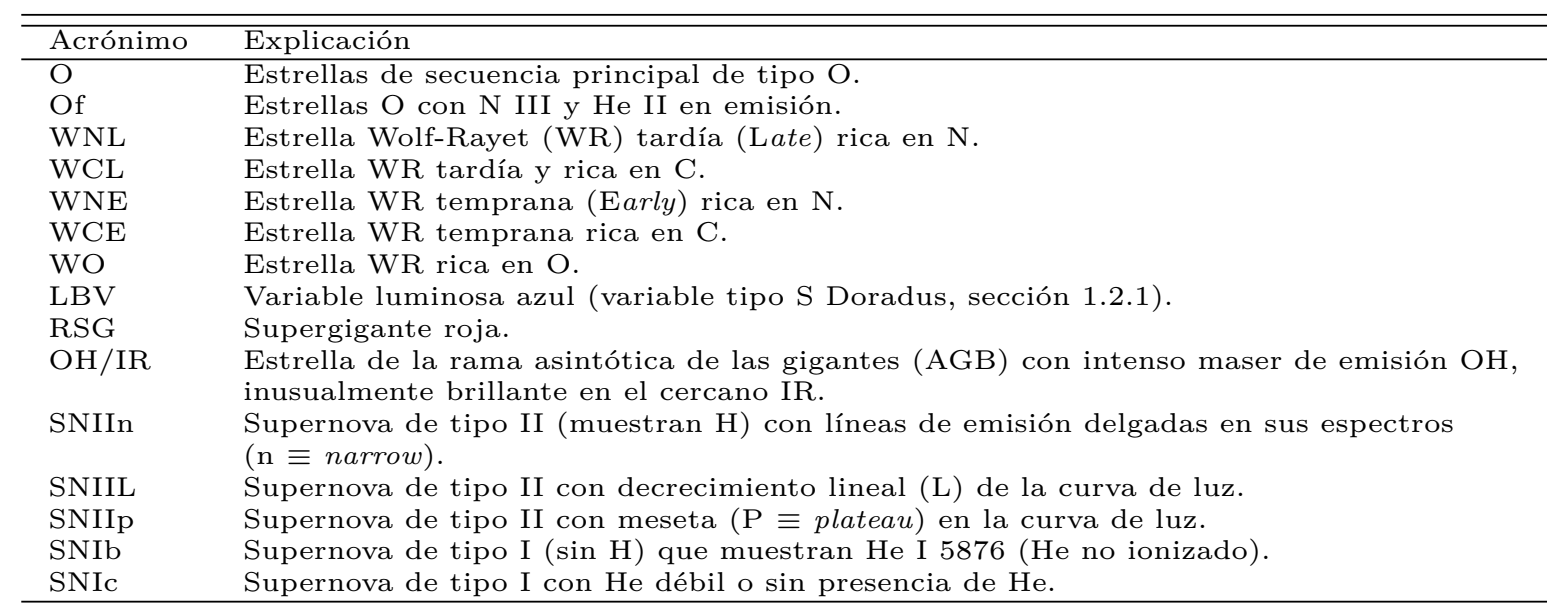

Tabla 1.1. Abreviaturas usadas en las secuencias evolutivas.

- Mayor de 40 a $60 \mathrm{M}_{\odot}$ (Siempre azules): Las altas tasas de pérdida de masa remueven suficiente material como para que estas estrellas pierdan sus envolturas 
en la secuencia principal o en la etapa de supergigante B como LBVs. Estas estrellas nunca se transforman en supergigantes rojas. Los posibles escenarios son:

$$
\begin{gathered}
\mathbf{M}>\mathbf{9 0} \mathbf{M}_{\odot}: \mathrm{O} \rightarrow \mathrm{Of} \rightarrow \mathrm{WNL} \rightarrow(\mathrm{WNE}) \rightarrow \mathrm{WCL} \rightarrow \mathrm{WCE} \rightarrow \mathrm{SN}(\text { Hipernova?) } \\
\mathbf{M}>\mathbf{6 0} \text { a } \mathbf{9 0} \mathbf{M}_{\odot}: \mathrm{O} \rightarrow \mathrm{Of} / \mathrm{WNL} \leftrightarrow \mathrm{WNL}(\text { pobre } \mathrm{en} \mathrm{H}) \rightarrow \mathrm{WCL}-\mathrm{E} \rightarrow \mathrm{SN}(\mathrm{SNIIn} ?) \\
\mathbf{M}>\mathbf{4 0} \text { a } \mathbf{6 0} \mathbf{M}_{\odot}: \mathrm{O} \rightarrow \mathbf{B S G} \rightarrow \mathrm{LBV} \leftrightarrow \mathrm{WNL} \rightarrow \mathrm{WCL}-\mathrm{E} \rightarrow \mathrm{SN}(\mathrm{SNIb}) \\
\text { WCL-E } \rightarrow \mathrm{WO} \rightarrow \mathrm{SN}(\mathrm{SNIc})
\end{gathered}
$$

- Entre 40 y $30 \mathbf{M}_{\odot}$ (Azules - Rojas - Azules): Las estrellas sólo pierden una fracción de sus envolturas en la secuencia principal. Luego evolucionan al estado de supergigante roja, donde la emisión de masa hace que pierdan sus envolturas. $\mathrm{Al}$ quedar con sus regiones internas expuestas son observadas como estrellas WR.

$$
\begin{gathered}
\mathrm{M}>\mathbf{3 0} \text { a } 4 \mathbf{4 0} \mathbf{M}_{\odot}: \mathrm{O} \rightarrow \mathrm{BSG} \rightarrow \mathrm{RSG} \rightarrow \mathrm{WNE} \rightarrow \mathrm{WCE} \rightarrow \mathrm{SN}(\mathrm{SNIb}) \\
\mathrm{OH} / \mathrm{IR} \leftrightarrow \mathrm{LBV} ?
\end{gathered}
$$

- Entre 30 y $25 \mathbf{M}_{\odot}$ (Azules - Rojas): Estas estrellas pierden masa en todas sus etapas, pero no en cantidad suficiente como para alterar su evolución posterior, evitando que retornen a la región azul del diagrama HR. La pérdida de masa modifica los tiempos de escala en varias fases y la composición química.

$$
\begin{aligned}
& \text { M>25 a } 30 \mathbf{M}_{\odot}: \mathrm{O} \rightarrow(\mathbf{B S G}) \rightarrow \mathrm{RSG} \rightarrow \mathrm{BSG} \leftrightarrow \mathrm{RSG} \rightarrow \text { SNIIL } \\
& \mathrm{M}>10 \text { a } 25 \mathbf{M}_{\odot}: \mathrm{O} \rightarrow \mathrm{RSG} \rightarrow(\text { Lazo de Cefeida) } \rightarrow \text { RSG } \rightarrow \text { SNIIp }
\end{aligned}
$$

\subsubsection{Parámetros fundamentales de las supergigantes $B$}

En esta sección se brinda un rango orientativo de los valores de los parámetros fundamentales de las supergigantes B tempranas, junto con una breve reseña sobre los métodos específicos usados para determinar cada parámetro.

La mayoría de los trabajos publicados que procuran estimar estos parámetros, se basan en el modelado de las características observadas de la fotosfera y del viento de las estrellas (líneas espectrales o continuo de radiación), mediante códigos de cálculo (ver un resumen sobre los mismos en la sección 5.2). Casi todos estos códigos han sido desarrollados en el marco de la teoría de vientos impulsados por radiación, la cual se detalla en el capítulo 2 .

Entre los numerosos estudios aplicados a las supergigantes B, pueden mencionarse como ejemplos, los trabajos de McErlean et al. (1999), que usa el código TLUSTY; el análisis de Searle et al. (2008), que combina los códigos TLUSTY (para la fotosfera) y CMFGEN (para el viento); y el trabajo de Markova \& Puls (2008), que usa el código FASTWIND (fotosfera y viento). Existen también métodos alternativos para calibrar algunos parámetros, como es el caso de la aplicación del sistema espectro fotométrico BCD (Barbier-Chalonge-Divan) que, mediante la medición precisa de la altura y las características del salto de Balmer, se emplea para calibrar las temperaturas efectivas estelares (Zorec et al. 2009).

En la Tabla 1.2 se reunen algunos valores que surgen de las calibraciones de los parámetros fundamentales de las supergigantes B. Resulta notorio el cambio en temperatura efectiva entre los primeros tipos espectrales (de B0 a B1). Estas diferencias 


\begin{tabular}{llllll}
\hline \hline T.E. & $\begin{array}{l}T_{\text {eff }} \\
{[k K]}\end{array}$ & $\log g$ & $\begin{array}{l}R_{*} \\
{\left[R_{\odot}\right]}\end{array}$ & $\begin{array}{c}M_{*} \\
{\left[M_{\odot}\right]}\end{array}$ & $\begin{array}{l}\log \mathrm{L}_{*} \\
{\left[\mathrm{~L}_{\odot}\right]}\end{array}$ \\
\hline B0 Ia & 28.1 & 2.99 & 26.9 & 25 & 5.60 \\
B0 Ib & 29.7 & 3.24 & 23.8 & 37 & 5.66 \\
B1 Ia & 22.0 & 2.41 & 36.5 & 12 & 5.44 \\
B1 Ib & 21.7 & 2.67 & 34.9 & 22 & 5.38 \\
B2 Ia & 18.3 & 2.32 & 51.0 & 19 & 5.41 \\
B2 Ib & 18.1 & 2.46 & 44.4 & 21 & 5.27 \\
B3 Ia & 16.4 & 2.16 & 60.4 & 19 & 5.37 \\
B3 Ib & 17.5 & 2.39 & 45.5 & 19 & 5.23 \\
B4 Ia & 15.8 & 2.06 & 63.5 & 16 & 5.34 \\
B4 Ib & 17.4 & 2.27 & 43.2 & 13 & 5.18 \\
B5 Ia & 15.7 & 2.03 & 63.0 & 15 & 5.33 \\
B5 Ib & 15.2 & 2.11 & 51.7 & 13 & 5.09 \\
\hline B5 Ib & 14.8 & 2.60 & 34 & 17 & 4.70 \\
B6 Ia* & 15.0 & 2.00 & $\ldots$ & $\ldots$ & 4.92 \\
B8 Iab & 13.0 & 1.70 & 68 & 9 & 5.08 \\
B9 Ia* & 11.5 & 2.00 & $\ldots$ & $\ldots$ & 4.88 \\
B9 Iab & 11.8 & 2.18 & 59 & 19 & 4.79 \\
\hline
\end{tabular}

Tabla 1.2. Extracto de la calibración de parámetros fundamentales para las supergigantes B tempranas. Hasta el tipo espectral B5, se presentan los resultados de Searle et al. (2008). A partir del tipo espectral B5, se adicionan algunos valores obtenidos para estrellas tardías individuales de Markova \& Puls (2008) y McErlean et al. (1999) $(\operatorname{marcados} \operatorname{con} *)$.

ponen en evidencia la gran variedad de condiciones físicas que se agrupan bajo la denominación "supergigantes B".

Respecto a la rotación de las estrellas supergigantes B, cabe mencionar que prácticamente todas las estrellas masivas comienzan su evolución como rotadores rápidos, y permanecen rotando con velocidades significativas durante todas sus etapas posteriores (Puls et al. 2008b). Sin embargo, de acuerdo a ese trabajo, la velocidad de rotación disminuye conforme evolucionan, ya sea por el incremento en sus radios como por la pérdida de sus momentos angulares a través del viento. En la secuencia principal, las estrellas de tipo $\mathrm{O}$ son los rotadores más rápidos conocidos (con valores de $v \sin i$ de hasta $400 \mathrm{~km} \mathrm{~s}^{-1}$ ), mientras que las supergigantes B rotan mucho más lento (con valores de $v \sin i \lesssim 50 \mathrm{~km} \mathrm{~s}^{-1}$ ). Como comparación, la velocidad de rotación del Sol en el ecuador es de apenas $2 \mathrm{~km} \mathrm{~s}^{-1}$.

A continuación se resume la metodología típica específica que se empleó para estimar cada uno de los parámetros fundamentales de la tabla 1.2 ;

- Temperatura efectiva: Para medir la temperatura efectiva $\left(T_{\text {eff }}\right)$ se usan las líneas de Si, cuya abundancia no es modificada por elementos provenientes del interior estelar en esas etapas evolutivas. Para el rango de supergigantes B0 a B2 se usan las líneas: Si IV $4089 \AA$ y Si III 4552, 4568 y $4575 \AA$. La primera línea 
decrece en intensidad al disminuir la temperatura efectiva, y se puede usar como sensor de temperatura para $\mathrm{T}_{\text {eff }}>18000 \mathrm{~K}$ (B2.5). En el tipo espectral B2.5, esta línea se hace muy débil y se la reemplaza por las líneas del doblete de Si II 4128-30 A, las que se usan hasta el tipo espectral B9, en conjunción con las líneas de Si III 4552, 4568 y $4575 \AA$. Las líneas de He I en 4144, 4387, 4471 y $4713 \AA$, junto con la línea de Mg II en $4481 \AA$, pueden usarse como indicadores secundarios de temperatura (y también de luminosidad). También se puede usar el método BCD para estimar los valores de $\mathrm{T}_{\text {eff }}$.

- Gravedad superficial: La gravedad superficial (log g) se estima en base al ajuste de la línea $\mathrm{H} \gamma$ y, usada como estimador secundario, la línea $\mathrm{H} \delta$. Las líneas $\mathrm{H} \alpha$ y $\mathrm{H} \beta$ usualmente están muy contaminadas por emisión generada en el viento, por lo cual no pueden considerarse como indicadores fiables de la gravedad.

- Luminosidad: Para la clasificación en luminosidad en supergigantes B tempranas, se usa el cociente de las líneas de Si IV $4089 \AA$ y He I 4026, 4121 y/o 4144 A. Para las más tardías que B1, se usa el cociente de Si III 4552, 4568 y 4575 $\AA$ respecto a He I $4387 \AA$.

- Radios y masas: Las calibraciones de los radios y de las masas son derivadas a partir de un ajuste lineal en los planos $\log \mathrm{T}_{\text {eff }}$ vs. $\log \mathrm{L}_{*} \mathrm{y} \log \mathrm{T}_{\text {eff }}$ versus $\log \mathrm{g}$. Como alternativa menos precisa, puede determinarse el radio estelar a partir de la fotometría, corregida por enrojecimiento, de la distancia y del tipo espectral, usando calibraciones previas de las temperaturas efectivas y las correcciones bolométricas.

- Rotación: De acuerdo a Ryans et al. (2002), para estimar v sin $i$ se usan dos métodos básicos. El primero consiste en la medición de los anchos equivalentes de líneas ensanchadas por rotación y la generación de perfiles teóricos de la misma intensidad usando modelos NLTE (fuera de equilibrio termodinámico local). El segundo hace uso de la transformada de Fourier de los perfiles rotacionales (Gray 2005). La distribución de los valores de $v \sin i$ para las estrellas tempranas puede verse en la figura 1.9 .

Es importante considerar que los perfiles de línea en las supergigantes B generalmente tienen otros mecanismos de ensanchamiento, entre los que destaca la llamada macro-turbulencia (ver sección 2.6.4). Por esta razón las mediciones de $v \sin i$ tienen considerable inexactitud.

La rotación de las estrellas masivas tiene una estrecha relación con la metalicidad de las estrellas, tal es el resultado que arrojan los análisis del relevamiento VLTFLAMES (Dufton et al. 2006, Mokiem et al. 2006; Hunter et al. 2008) de estrellas OB en cúmulos de la galaxia, Nube Menor de Magallanes (Small Magellanic Cloud, SMC) y Nube Mayor de Magallanes (Large Magellanic Cloud, LMC). Para una muestra de estrellas B, se encuentra una clara diferencia en la rotación, siendo que los objetos de la SMC (baja metalicidad) rotan más rápido. Se argumenta que la diferencia se debe a que estas estrellas poseen vientos más débiles, por lo que pierden menos momento angular, y tienen menores radios. La distribución 
de velocidades de rotación puede aproximarse con una gaussiana centrada en 100 $\mathrm{km} \mathrm{s}^{-1}$ para la LMC y $175 \mathrm{~km} \mathrm{~s}^{-1}$ para la SMC (Hunter et al. 2008).

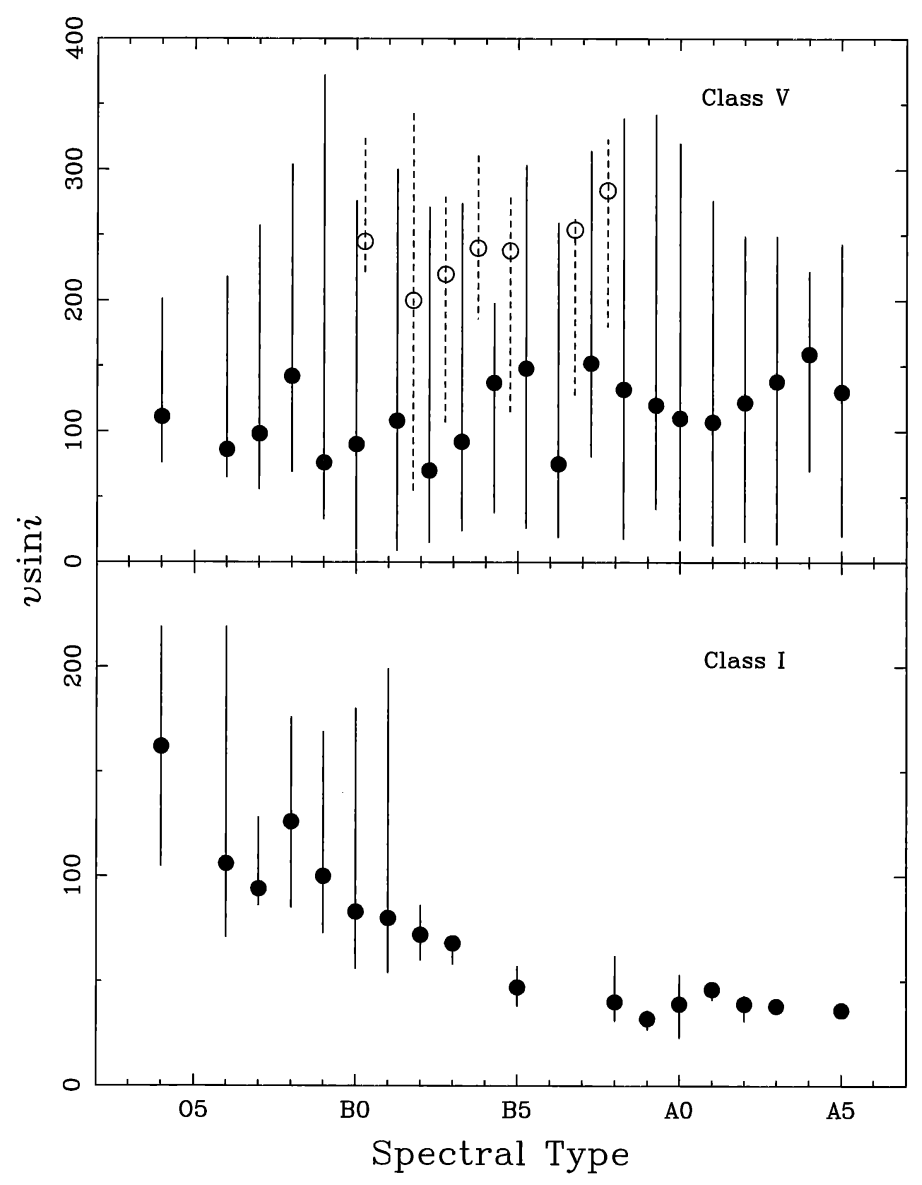

Figura 1.9. Distribución de $v$ sin $i$ en función del tipo espectral para estrellas galácticas tempranas. La distribución para las enanas está en el panel superior, mientras que el caso de las supergigantes está en el panel inferior (nótese la diferencia de escalas en las ordenadas). Las barras verticales representan el rango total. Los círculos huecos corresponden a las estrellas Be, los más altos rotadores. Figura tomada de Howarth (2004).

\subsubsection{Parámetros globales de los vientos estelares}

A partir de las evidencias observacionales disponibles que ponen en manifiesto la presencia de vientos en las estrellas tempranas (sección 1.1.1), se pueden estimar, con mayor o menor precisión, los dos parámetros globales del viento: la tasa de pérdida de masa $(\dot{M})$ y la velocidad terminal del viento $\left(v_{\infty}\right)$.

La tasa de pérdida de masa es la cantidad de materia expulsada por la estrella en la unidad de tiempo, y se mide habitualmente en $M_{\odot}$ año ${ }^{-1}$, donde $M_{\odot}$ es la masa del Sol. La tasa de pérdida de masa para una estrella como el Sol (G2 V) es pequeña, del orden de $10^{-14} M_{\odot}$ año ${ }^{-1}$, mientras que, en el otro extremo, una estrella WR tiene una tasa de pérdida de masa típica del orden de $10^{-5} M_{\odot}$ año ${ }^{-1}$. En el caso de las estrellas 
supergigantes $\mathrm{B}$, las tasas son de uno a dos órdenes de magnitud menor, entre $10^{-6} \mathrm{y}$ $10^{-7} M_{\odot}$ año ${ }^{-1}$.

La velocidad terminal del viento es el valor máximo de la velocidad del material que se alcanza a grandes distancias de la estrella. En la teoría estándar, el viento estelar es modelado como un flujo estacionario y homogéneo de materia, con simetría esférica, que se inicia en las capas más altas de la fotosfera, donde prevalece un estado de quasiequilibrio hidrostático. A partir de allí, el material es acelerado monótonamente por absorción de momento de los fotones. Esto da lugar a una sucesión de diferentes gradientes de velocidad, hasta que se alcanza la velocidad terminal $v_{\infty}$, donde el gradiente se anula. La aceleración del material, originada por la fuerza del campo de radiación, se interrumpe a grandes distancias debido a que disminuye sensiblemente la eficiencia en la absorción de momento por parte del gas. De esta manera, el viento alcanza el medio interestelar con una velocidad constante $v_{\infty}$.

Los vientos pueden tener velocidades terminales muy bajas, como es el caso de las estrellas supergigantes tardías con valores del orden de $10 \mathrm{~km} \mathrm{~s}^{-1}$, hasta velocidades de $3000 \mathrm{~km} \mathrm{~s}^{-1}$ para los flujos supersónicos de las estrellas tempranas más luminosas. En el caso de las estrellas supergigantes $\mathrm{B}$, los valores típicos de $v_{\infty}$ están comprendidas entre $2000 \mathrm{~km} \mathrm{~s}^{-1}$, para las estrellas más tempranas, y $100 \mathrm{~km} \mathrm{~s}^{-1}$ para las más tardías.

Los valores de $\dot{M}$ y $v_{\infty}$ dependen, en primera instancia, de los parámetros fundamentales de la estrella tales como la temperatura efectiva $\left(\mathrm{T}_{\text {eff }}\right)$, la gravedad superficial $(\log g)$, el radio $\left(R_{*}\right)$, la rotación y la metalicidad $(Z)$. Ya que el viento de estas estrellas se origina por la transferencia de momento del campo de radiación estelar a los iones disponibles en la atmósfera estelar, estos parámetros están condicionados por las propiedades del gas. Por lo tanto, son críticas las características tales como la capacidad de los iones para absorber momento (dado, por ejemplo, por la cantidad de líneas disponibles de acuerdo a la composición iónica), de la estructura atómica de los iones y de su estado de excitación e ionización, entre otros factores.

De acuerdo a Puls et al. (1996), tanto la tasa de pérdida de masa como las velocidades terminales no son únicos, sino que diferentes combinaciones de los mismos con distintos radios estelares, podrían dar modelos con las mismas características espectrales (es decir perfiles de línea con los mismos anchos equivalentes e incluso, forma). Para evitar esta multiplicidad en los modelos se han propuesto dos cantidades: Q para las líneas de resonancia con opacidad proporcional a $\rho$, y Q para líneas de recombinación (por ejemplo $\mathrm{H} \alpha$ ), cuyas opacidades son proporcionales a $\rho^{2}$. Tales cantidades son llamadas invariantes de profundidad óptica, que tienen la forma:

$$
\left\{\begin{array}{l}
Q_{\text {res }}=\frac{\dot{M}}{R_{*} v_{\infty}^{2}}, \\
Q=\frac{\dot{M}}{\left(R_{*} v_{\infty}\right)^{1.5}} .
\end{array}\right.
$$

De este modo, los valores de $\dot{M}$ pueden obtenerse por separado, cuando se conocen $\mathrm{v}_{\infty} \mathrm{y} \mathrm{R}_{*}$ individualmente. De lo contrario, el resultado de los ajustes de los perfiles de líneas obtenidos por modelos no permiten la medición individual de cada parámetro del viento, sino del invariante correspondiente (Puls et al. 2008b). 


\subsubsection{Estimación de $\dot{M}$}

El parámetro $\dot{M}$, tal como fue definido en la sección anterior, no es medible directamente a partir de la observación. Su estimación está estrechamente vinculada a la adopción de un modelo que dé cuenta de los procesos hidrodinámicos del viento, considerando las fuerzas intervinientes y la reacción del material generada por sus características propias, tales como la composición química o el estado de ionización. Además, si la medición de $\dot{M}$ se realiza por medio de la comparación de características observables del espectro estelar tales como los excesos de emisión del continuo en diferentes frecuencias, o en la comparación de las líneas espectrales observadas con sus análogos sintéticos obtenidos a partir de soluciones hidrodinámicas generadas por códigos de cálculo de vientos estelares, se requiere de la evaluación del transporte de radiación para medios en movimiento. La confiabilidad de los resultados dependerá del modelo elegido, de las aproximaciones usadas y de la calidad de las observaciones.

De acuerdo a esto, la medición de los excesos en el continuo de radio, submilimétrico y también en el IR, mencionados en la sección 1.1.1, son la manera más simple para la estimación de $\dot{M}$. Esto se debe a que el modelado de estas cantidades está basado en mecanismos atómicos y de transporte de radiación considerablemente simples. Por el contrario, el ajuste de líneas espectrales observadas mediante perfiles sintéticos, es un proceso más complejo ya que involucra el cálculo de poblaciones atómicas en NLTE, mecanismos de ensanchamiento de perfiles, el transporte de radiación en medios en movimientos, etc.

Se han realizado numerosas mediciones de las tasas de pérdida de masa de las estrellas OB a partir de sus flujos en radio, como ejemplo, Gabler et al. (1989) o el catálogo de Wendker (1987), pero como estas estrellas son fuentes en radio débiles, sólo se han medido flujos de estrellas relativamente cercanas. Otro inconveniente de estas determinaciones es la contaminación de radiación no térmica en el continuo de radio que consiste, básicamente, en radiación sincrotrón de electrones relativistas. Su origen está asociado a las regiones con generación de rayos $\mathrm{X}$ que pueden deberse a la colisión de vientos en binarias, la acreción de material en objetos compactos, la formación de ondas de choque (shocks) en el viento o la actividad en campos magnéticos, entre otros. Otra fuente de error en esta determinación se produce cuando las hipótesis básicas del modelo no se adecuan convenientemente a la estrella, como es el caso de los apartamientos de la simetría esférica o la falta de homogeneidad del viento (por ejemplo, por la presencia de grumos de material, fenómeno llamado clumping, sección 2.6.8.

Las estrellas con vientos intensos pueden presentar excesos en el IR, los que también han sido usados para derivar $\dot{M}$ (por ejemplo, Bertout et al. 1985). En algunos casos se ha encontrado que los excesos en el IR son muy intensos y conducen a mediciones de $\dot{M}$ muy grandes respecto a las obtenidas a partir del continuo de radio o de $\mathrm{H} \alpha$. En general, se atribuyen estas discrepancias a la variabilidad fotométrica de las supergigantes B, o a la presencia de clumps en los vientos.

La intensidad de los perfiles P Cygni en el UV también se usa para diagnosticar la tasa de pérdida de masa. El método más usado para analizar estos perfiles es el llamado método SEI (Lamers et al. 1987), que fue aplicado por primer vez por Groenewegen et al. (1989). En estos casos, sólo puede estimarse el producto $\dot{M} q$, donde $q$ es la fracción de ionización correspondiente al ion de la línea en particular (Puls et al. 2008a). 
Cuando las líneas están saturadas, en estrellas de vientos intensos, sólo puede obtenerse entonces, un límite inferior para el valor de $\dot{M}$.

Por último, puede usarse la emisión en la línea $\mathrm{H} \alpha$ para medir $\dot{M}$, mediante el ajuste de perfiles sintéticos obtenidos de códigos NLTE de atmósfera. Los primeros trabajos en aplicar esta técnica fueron los de Klein \& Castor (1978), que luego fue aplicada en numerosas oportunidades (por ejemplo, Leitherer 1988; Drew 1990; Lamers \& Leitherer 1993: Crowther et al. 2006; Markova \& Puls 2008; Searle et al.|2008).

De acuerdo a su origen, cada una de las características citadas de los espectros, muestrea diferentes partes del viento. $\mathrm{H} \alpha$ diagnostica preferentemente la región más densa e interna del viento, próxima a la estrella y con una rápida aceleración. La emisión libre-libre en ondas de radio del viento evalúa las regiones más distantes y rarificadas, que se mueven con una velocidad casi constante. Las líneas de resonancia del UV incorporan contribuciones de ambas regiones, registrando las partes intermedias. De acuerdo a Fullerton et al. (2006), el uso de las líneas de resonancia en el UV como estimadores de $M$ tiene algunas dificultades que requieren un modelado más preciso de las condiciones físicas del viento. Este problema surge porque la intensidad de estas líneas está determinado por la profundidad óptica radial del viento, $\tau \propto \dot{M} q_{i} A_{\mathrm{E}}$, donde $A_{\mathrm{E}}$ y $q_{i}$ son la abundancia del elemento E y su fracción de ionización para el estado $i$, respectivamente. De este modo, si se modela el viento con un ion dominante $q_{i} \approx 1$ de abundancia conocida, puede estimarse $\dot{M}$ directamente. Esto ocurre para líneas de los iones dominantes saturadas en vientos suficientemente densos. De lo contrario, las líneas de resonancia en el UV de iones con fracción de ionización $q_{i} \lesssim 10^{-3}$ sólo darán una medida de $\dot{M} q$. En esos casos, es necesario estimar la fracción de ionización $q_{i}$ muy precisamente para derivar la tasa de pérdida de masa adecuadamente (Lamers et al. 1999).

\subsubsection{Estimación de $v_{\infty}$}

La velocidad terminal de las estrellas tempranas puede medirse directamente en los perfiles P Cygni de las líneas de resonancia de iones tales como el CIV o Si IV en el UV (por ejemplo, Lamers et al. 1995). Estas mediciones son confiables cuando las líneas presentan perfiles P Cygni saturados (figura 1.10). En estos perfiles, las componentes en absorción son tan intensas que han bloqueado toda la radiación del continuo estelar en el rango de velocidades $v=0 \ldots v_{\infty}$. En estos casos, la velocidad terminal del viento se mide directamente a partir de la posición del borde azul, casi vertical, de esta componente, la cual corresponde al máximo desplazamiento Doppler. Si el perfil no está saturado, sólo se tendrá una cota inferior para dicho parámetro.

La determinación de la tasa de pérdida de masa a partir de un perfil P Cygni es menos confiable que la que se obtiene de los excesos en IR y radio. Esto se debe a que, en su determinación, se debe aplicar un modelo teórico en el cual es necesario el conocimiento preciso de la ley de velocidades, así como también algunas características del medio, por ejemplo, las poblaciones de los iones en el equilibrio de ionización, o la abundancia relativa de los elementos. Por lo general, la determinación se realiza con un ajuste o fitting de los perfiles observados con perfiles teóricos.

Algunas comparaciones entre los valores de $\dot{M}$ obtenidos a partir de los excesos de radio y los determinados a partir de la línea $\mathrm{H} \alpha$ se encuentran en los trabajos de 

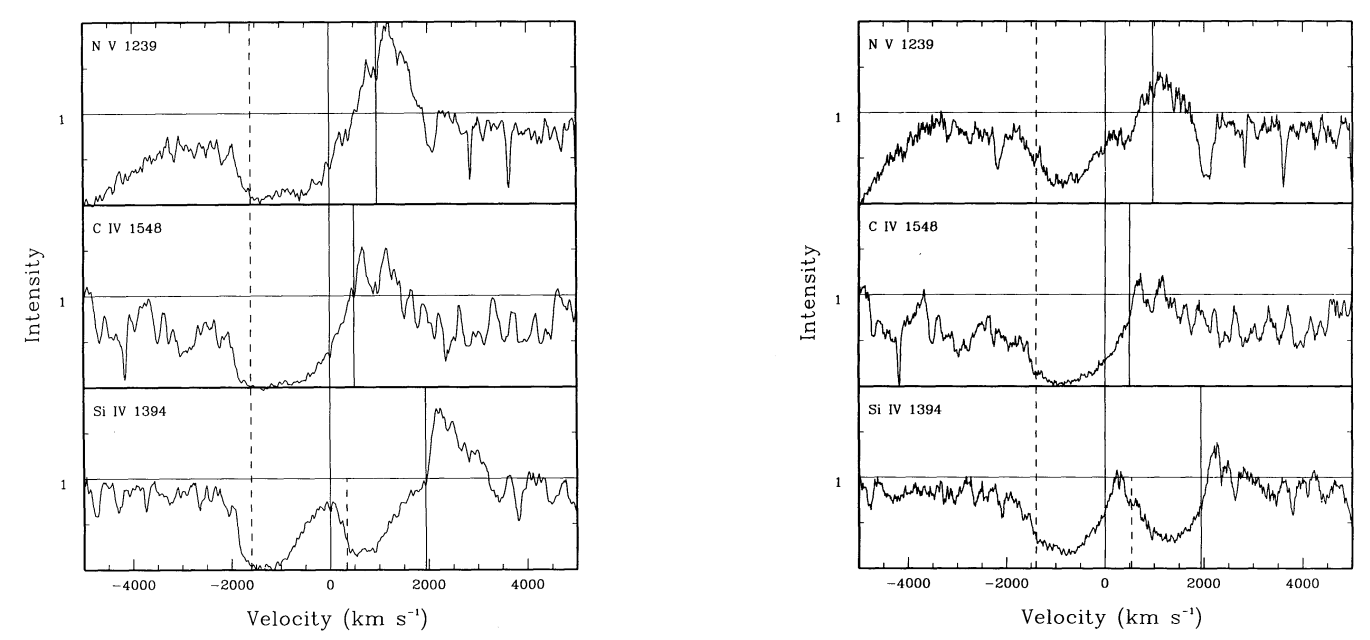

Figura 1.10. Ejemplos de la medición de $v_{\infty}$ (indicada con la línea a rayas) respecto a la velocidad correspondiente a la longitud de onda de reposo de cada línea de un doblete. En el gráfico izquierdo se puede ver la determinación simultánea de $v_{\infty}$ en perfiles P Cygni saturados de tres iones diferentes. En el panel de la derecha se observa la medición en perfiles no saturados, lo cual disminuye la certeza de los valores. Tomado de Lamers et al. (1995).

Lamers \& Leitherer (1993) y de Puls et al. (1996). En muchos casos, aparecen discrepancias que han sido atribuidas a la variabilidad fotométrica de los objetos estudiados, particularmente, en el caso de las supergigantes B (sección 2.6.7). Se ha propuesto una explicación alternativa que se basa en que el viento no es homogéneo. Si hubiera grumos de materia o clumps, esto incrementaría los excesos IR generando una inconsistencia con los flujos medidos en $\mathrm{H} \alpha$, considerando que la opacidad de $\mathrm{H} \alpha$ es proporcional al cuadrado de la densidad $\left(\rho^{2}\right)$. En el empleo de $\mathrm{H} \alpha$ como indicador de $\dot{M}$ hay algunas fuentes adicionales de incerteza como, por ejemplo, una superposición (blend) con una línea de He II en el ala azul en el caso de las estrellas O y las B tempranas.

Para usar líneas en el óptico o en el IR, que tienen su origen en la fotosfera o que pueden estar contaminadas por contribuciones fotosféricas, es necesario considerar un modelo de viento unificado, es decir, aquel en el que se calcula la fotosfera y el viento conjuntamente, que se presenta en la sección 2.3. El marco teórico para el estudio de las líneas en el IR es el mismo que para las líneas en el UV. Sin embargo, prácticamente todas las líneas en el IR se forman a lo largo del viento y muestrean distintas regiones del mismo, dando información sobre el campo de velocidades. Aún vientos muy tenues con tasas de pérdida de masa extremadamente bajas pueden producir intensas líneas en emisión, debido a los apartamientos de LTE causados por la importante contribución de la emisión estimulada producida en el viento (Najarro et al. 1998). 


\subsection{Breve reseña histórica del modelado teórico de los vientos estelares}

La idea de que el material puede ser eyectado por la estrella a partir de la presión de radiación sobre el material fue sugerida por primera vez por Milne (1924, 1926). En el segundo trabajo citado, Milne propuso que el corrimiento Doppler debido al movimiento de los iones podía extender la acción de la fuerza de radiación, evitando la atenuación de la radiación del continuo en capas más internas de la atmósfera.

El trabajo de Lucy \& Solomon (1970) fue uno de los primeros en estudiar y cuantificar el fenómeno de los vientos impulsados por la radiación en las líneas. Los autores demostraron que la absorción de momento del campo de radiación de una estrella OB en un pequeño conjunto de líneas intensas de resonancia en el UV, cerca del máximo de la distribución de energía estelar, puede ser suficiente para que el material sea acelerado y escape del campo gravitatorio estelar. Como en este trabajo sólo se considera la suma de unas pocas líneas ópticamente gruesas, los valores de las tasas de pérdida de masa resultan insuficientes en comparación con los derivados de las observaciones.

El mayor avance para la teoría de vientos estelares fue realizado por Castor, Abbott, \& Klein (1975, teoría CAK), en el que demostraron que la contribución de muchas líneas débiles a la par de unas pocas líneas intensas, podía sobrepasar en un factor 100 a la sola contribución de las líneas intensas de resonancia, generando pérdidas de masa acordes a las medidas. En base a una lista de líneas del C III, pudieron obtener por primera vez, una solución hidrodinámica analítica y autoconsistente para los vientos estelares, compatible con las características observacionales de los mismos. Castor et al. (1975) propusieron una parametrización sencilla pero muy eficaz, para representar a la aceleración de radiación dada por la transferencia de momento del campo radiativo en un conjunto muy grande de líneas espectrales. Esta parametrización se basa en dos parámetros, $k$ y $\alpha$, que miden la cantidad de líneas intervinientes y la capacidad de los iones para absorber momento, respectivamente (sección 2.2.4).

Este estudio fue complementado por el trabajo de Abbott (1982b), donde se presentaron cálculos mejorados de la aceleración en la línea, incorporando el parámetro $\delta$ para considerar la estructura de ionización del viento (sección 2.2.6. Para obtener la aceleración de las líneas, Abbott (1982b) mejoró sensiblemente el cálculo de las opacidades, usando una vasta lista de líneas espectrales con sus valores $g f$ (producto de los pesos estadísticos y las intensidades de oscilador para las transiciones), extendiéndose a las seis primeras etapas de ionización de los elementos que van desde el H hasta el Zn. En base a esta información, construyó una extensa tabulación para los parámetros de la fuerza de radiación, la cual se extiende en un amplio rango de temperaturas efectivas, densidades y abundancias químicas.

Tanto Castor et al. (1975) como Abbott (1982b), obtuvieron las soluciones analíticas para las ecuaciones hidrodinámicas de un viento impulsado por radiación en el caso de una estrella puntual (es decir, con un flujo radial de fotones). En dos trabajos simultáneos, Friend \& Abbott (1986) y Pauldrach, Puls, \& Kudritzki (1986) ampliaron la teoría, obteniendo las soluciones hidrodinámicas con la incorporación a la aceleración de radiación de una corrección que da cuenta de la influencia de los fotones no radiales, dando lugar a la llamada teoría $m$-CAK o CAK modificada (sección 2.2.8). En el trabajo de Pauldrach et al. (1986), los autores compararon la fuerza de radiación cal- 
culada previamente en la llamada aproximación de Sobolev (en la que la radiación sólo actúa en regiones reducidas de resonancia y que es válida para flujos de alta velocidad, sección 2.2.3 con los resultados obtenidos con la resolución del transporte de radiación más general, en el marco solidario al fluido (CMF, comoving-frame), demostrando la eficacia de esa aproximación.

A través de numerosas contribuciones se fue construyendo la teoría estándar de vientos impulsados por radiación. Esta teoría puede predecir la estructura básica de los vientos de muchas estrellas tempranas aproximando, con distintos grados de exactitud, las características generales observadas en sus espectros. La teoría estándar de vientos impulsados por radiación ha sido muy exitosa para representar la estructura de los vientos de las estrellas O tanto enanas como supergigantes, así como también, al menos parcialmente, los espectros de las B más tempranas. Su logro más importante es la elaboración de la relación Momento del Viento-Luminosidad (WLR, sección 2.5.1 Kudritzki et al. 1995, Puls et al. 1996), que vincula la tasa de transferencia de momento, calculada a partir de los parámetros globales del viento, con la luminosidad de la estrella. De este modo, la WLR permite determinar distancias galácticas y extragalácticas para estrellas supergigantes tempranas, mediante el modelado de las características espectrales distintivas de los vientos (Kudritzki et al. 1999).

En el marco de la teoría estándar de vientos impulsados por radiación, se han desarrollado códigos de cálculo muy elaborados, capaces de predecir la apariencia del continuo y de las líneas espectrales. Los códigos más completos y mayor número de resultados son: FASTWIND (Santolaya-Rey et al. 1997; Puls et al. 2005; Rivero González et al. 2012), CMF-GEN (Hillier 1987; Hillier \& Miller 1998; Hillier \& Lanz 2001; Hillier 2012), WM-basic (Pauldrach et al. 2001; Sternberg et al. 2003) y PoWR (Hamann \& Schmutz 1987; Todt et al. 2015). Sus características son resumidas en la sección 5.2 .

A pesar de estos esfuerzos, la teoría estándar de vientos impulsados por radiación no es suficiente para explicar la gran diversidad de características espectrales que presentan todas las estrellas masivas. Por ejemplo, los espectros de las estrellas supergigantes de subtipos espectrales B intermedios y tardíos, junto a las supergigantes A, que presentan importantes discrepancias respecto a las estimaciones de los parámetros globales del viento (por ejemplo, Verdugo et al. 1999, Crowther et al. 2006, Searle et al. 2008. Markova \& Puls 2008). Otro de los efectos no previstos por la teoría es el mecanismo de biestabilidad (sección 2.6.2), que es una transición discontinua en las características globales $\left(\dot{M}\right.$ y $\left.v_{\infty}\right)$ de los vientos para estrellas a ambos lados de un determinado valor de la temperatura efectiva. De acuerdo a Vink et al. (1999), la resolución de este problema requiere realizar un avance sobre algunas simplificaciones del modelo; para este caso, un cálculo más detallado del equilibrio de ionizaciones del Fe. Algunas de estas diferencias se presentan en la sección 2.6.7.

En otros aspectos, la teoría de vientos impulsados por radiación tiene limitada su capacidad de predicción por la propia adopción de aproximaciones matemáticas que se usan para simplificar los cálculos. Un ejemplo de estas aproximaciones es el uso extendido de una forma fija para representar el campo de velocidades en función de la distancia, que es la "ley $\beta$ " de velocidades (sección 2.1.1. ecuación 2.2. Si bien esa forma funcional para la velocidad puede obtenerse analíticamente con un valor de $\beta$ restringido a 0.5 , como solución de las ecuaciones hidrodinámicas para los vientos bajo ciertas circunstancias (Castor et al. 1975), el uso de esta ley se ha extendido sin ninguna 
justificación, para representar la generalidad de los vientos de las estrellas tempranas. De hecho se usan valores de $\beta$ tan altos como 3 (por ejemplo, Kudritzki et al. 1999 , Lefever et al. 2007).

Por estos motivos se han ido incorporando a los códigos, otros fenómenos y propiedades de las estrellas que pueden tener importantes efectos sobre sus vientos. Uno de estos fenómenos es el line-blocking/blanketing, que considera el bloqueo de la radiación fotosférica disponible para acelerar el viento por parte de un nutrido conjunto de líneas espectrales (line-blocking). Como reacción se produce una redistribución de la radiación en el continuo en otras frecuencias (blanketing).

También se han realizado considerables esfuerzos para incluir el fenómeno del clumping, ya que la presencia de grumos de material en el viento cambia considerablemente la manera en que la radiación se transmite a través del plasma, modificando su capacidad para acelerar el viento. Ejemplos del tratamiento de este efecto pueden encontrarse en muchos de los trabajos citados sobre los códigos. En general, su finalidad consiste en reducir las tasas de pérdida de masa para mejorar los ajustes (sección 2.6.7). Aunque existen muchas evidencias de la presencia de las inhomogeneidades, el tratamiento que se hace para tener en cuenta el fenómeno es fuertemente dirigido para mejorar los ajustes con las observaciones y dependen del modelo empleado.

Otra de las mejoras más importantes a la teoría de vientos impulsados por radiación consistió en la incorporación de la fuerza centrífuga producida por la rotación estelar a las ecuaciones hidrodinámicas (capítulo 3). Muchas estrellas supergigantes B rotan con velocidades que alcanzan los $100 \mathrm{~km} \mathrm{~s}^{-1}$, lo cual contribuye efectivamente a modificar el potencial gravitatorio estelar y por consiguiente, a sus vientos.

Curé (2004) descubrió que, con esta modificación, además de la conocida solución hidrodinámica de tipo m-CAK (a la que se nombrará como solución rápida en este trabajo), existe una nueva clase de solución para el viento, llamada solución $\Omega_{\text {lenta }}$ (sección 3.3.2). Esta clase de solución aparece cuando la estrella rota a altas velocidades, próximas a la velocidad de ruptura. Es habitual expresar la tasa rotacional $\Omega$ como el cociente $V_{\text {rot }} / V_{\text {break }}$, donde $V_{\text {rot }}$ es la velocidad de rotación en el ecuador estelar y $V_{\text {break }}$ es la velocidad de ruptura o velocidad a la cual debe girar una estrella para que su fuerza centrífuga equipare a su fuerza gravitatoria. La solución $\Omega_{\text {lenta }}$ aparece para valores de $\Omega \gtrsim 0.7-0.8$.

Comparada con la solución rápida, la solución $\Omega_{\text {lenta }}$ produce un viento más denso y lento, con baja $v_{\infty}$. De esta manera, se encontró que las ecuaciones hidrodinámicas para vientos impulsados por rotación, que son fuertemente no lineales, pueden originar más de un tipo de soluciones. Curé \& Rial (2004) estudiaron las características topológicas de la nueva solución y Araya et al. (2014) presentaron expresiones analíticas aproximadas para la aplicación de las soluciones.

Posteriormente, Curé et al. (2011) mostraron la existencia de otra solución hidrodinámica, la llamada solución $\delta_{\text {lenta }}$ (sección 3.3.2). La misma aparece cuando se consideran valores altos para el parámetro $\delta$ de la fuerza de radiación, que caracteriza a la ionización del material según el formalismo de la teoría CAK (Abbott 1982b; Friend \& Abbott 1986; Pauldrach et al. 1986, Puls et al. 2000). Esta solución comparte, en gran medida, las mismas características de la solución $\delta_{\text {lenta }}$, prediciendo vientos más densos con bajos valores para $v_{\infty}$. Sin embargo, a diferencia de la solución $\Omega_{\text {lenta }}$, la solución 
$\delta_{\text {lenta }}$ existe aún en el caso de rotación nula.

Resulta importante notar que, aunque las velocidades de los vientos modelados por las soluciones $\Omega_{\text {lenta }}$ y $\delta_{\text {lenta }}$ son notablemente más bajas que las correspondientes a las soluciones rápidas, los valores de $\dot{M}$ pueden ser mayores, del mismo orden o incluso menores. Esto implica que, para estos modelos, hay un importante reacomodamiento en la densidad del viento.

La solución $\Omega_{\text {lenta }}$ ha sido propuesta para explicar la formación de discos alrededor de estrellas Be (Curé 2004) y, en combinación con el mecanismo de bi-estabilidad (sección 2.6.2), para dar cuenta de los discos en torno a las estrellas B[e] (Curé et al. 2005); mientras que la solución $\delta_{\text {lenta }}$ puede representar con buena aproximación a los vientos de las estrellas supergigantes A (Curé et al.2011). El avance en la comprensión de estas nuevas soluciones y su posible aplicación para describir a los vientos de las estrellas masivas se está llevando a cabo. Esta tesis se enmarca, precisamente, en la exploración de estas nuevas herramientas teóricas para el análisis de los vientos estelares, en este caso, para las estrellas supergigantes B.

\subsection{Objetivos de esta tesis}

A pesar de sus notables avances (sección 2.5), la teoría estándar de vientos impulsados por radiación ha demostrado también sus limitaciones, ya que aún persisten muchas discrepancias entre la teoría y la observación, como se menciona en la sección anterior y se presenta en la sección 2.6.

Por otro lado, la incorporación de soluciones hidrodinámicas para estrellas en rotación por parte de Curé (2004) y Curé et al. (2011) abre nuevas líneas de investigación, que pueden contribuir a la comprensión de los vientos de las estrellas masivas. Estas soluciones aún no han sido exploradas completamente en sus propiedades, así como tampoco han sido evaluadas en su capacidad de modelar las observaciones de los vientos estelares.

Para llevar a cabo una indagación profunda de las posibilidades que propician estas soluciones, es necesario contar con herramientas potentes de cálculo, básicamente, códigos hidrodinámicos y de transporte de radiación preparados adecuadamente para analizar a las nuevas propiedades de las soluciones. Es necesario que estos códigos sean capaces de dar, a partir de los conceptos teóricos, elementos de análisis tales como los perfiles sintéticos de las líneas espectrales para su comparación con las observaciones.

Dado que el conjunto de las estrellas masivas es extremadamente rico y complejo, es necesario acotar el espacio de análisis a un subgrupo de objetos que asegure cierto nivel de heterogeneidad pero que, a su vez, comparta características comunes suceptibles de ser modeladas con la teoría estándar. Las estrellas supergigantes B constituyen un espacio ideal para este estudio ya que, en su extremo de altas temperaturas, guardan gran semejanza con las estrellas $\mathrm{O}$, para las cuales la teoría estándar ha tenido sus mayores aciertos. Por otro lado, en su grupo de tipos espectrales intermedios y tardíos, ofrece un gran desafío por la escasez de trabajos previos que modelen sus vientos, lo que revela su dificultad. A su vez, la variabilidad intrínseca y las dificultades que las supergigantes B ofrecen al modelado teórico, así como también la incerteza sobre su estado evolutivo, ofrecen un terreno fértil para nuevos descubrimientos. 
Por todo lo previamente expuesto, en esta tesis se intenta dar respuesta a los siguientes interrogantes:

- ¿Cuál es la relación entre la fuerza de radiación que produce la aceleración del viento y las soluciones lentas, y cuáles son los dominios de cada una de ellas en el espacio de los parámetros de la misma?

- ¿En qué condiciones puede prevalecer una clase de solución sobre otra?

- ¿Puede modelarse el viento de las estrellas supergigantes B con las soluciones lentas? ¿Son adecuadas estas soluciones?

- En el caso en que la respuesta anterior sea positiva, ¿qué consecuencias tiene esta posibilidad sobre las relaciones más importantes de la teoría de vientos impulsados por radiación, tales como la relación WLR?

- ¿Habría una manera de explorar la variabilidad de las estrellas supergigantes B en base a alternancia entre las soluciones que adopten sus vientos?

Para dar respuesta a estos interrogantes, en esta tesis se usa a la teoría estándar para vientos impulsados por radiación como soporte básico sobre el cual se adiciona la rotación estelar y se explora y evalúa, la potencialidad de las soluciones adicionales para dar cuenta de las características de los vientos en las estrellas tempranas y sus espectros resultantes.

En especial, entre las soluciones de tipo $\Omega_{\text {lenta }}, \delta_{\text {lenta }}$ las clásicas soluciones rápidas, no se conoce en detalle en qué entornos o condiciones físicas puede dominar una clase de solución sobre la otra y cuáles son los límites que separan cada uno de los dominios. De todas las soluciones, las $\delta_{\text {lenta }}$ son las soluciones que menos han sido exploradas, aún siendo las potencialmente más promisorias por no requerir objetos en alta rotación. Resulta claro que las condiciones de ionización de los vientos son extremadamente complejas (ver sección 1.1.1), lo cual puede permitir que se alcancen valores altos en el parámetro $\delta$, adaptando a los vientos a las condiciones de la solución $\delta_{\text {lenta }}$.

Como el análisis de las propiedades distintivas que la aplicación de estas soluciones pueden producir en el espectro aún no se ha realizado, es necesario obtener, de manera apropiada, estas nuevas soluciones y, a partir de ellas, extraer las características espectrales sintéticas correspondientes para ser comparadas con las observaciones. Con este motivo, en esta tesis se hace uso de dos códigos de cálculo: el código HYDWIND, para calcular rigurosamente y discriminar las tres soluciones hidrodinámicas posibles, y un código NLTE de atmósfera unificada, actualizado en el marco de la teoría estándar y adaptado para las nuevas soluciones, el código FASTWIND. Es necesario que este código pueda resolver convenientemente el transporte radiativo, tanto en la aproximación de Sobolev con continuo como, de manera más rigurosa, en el marco de referencia de los átomos en movimiento (comoving frame), de forma de evitar el uso de aproximaciones tales como la ley $\beta$ de velocidades, para usar en su lugar, las soluciones lentas de Curé. Por otro lado, para su aplicación a las atmósferas densas de estrellas de tipos espectrales más tardíos (entre las B), también es necesario adaptar este código a las condiciones de los campo de radiación, de las temperaturas y densidades en las 
que podrían darse inversiones de población de los iones, cuya ocurrencia conlleva a importantes problemas de convergencia en los códigos (sección 5.3).

Cabe aclarar que, en esta tesis, no se ha tenido en cuenta el tratamiento del clumping debido a que este fenómeno es altamente dependiente de los modelos aplicados y aquí, por el contrario, se busca comprender características más básicas subyacentes, concernientes a la ley de velocidades y de densidad, que las modificaciones generadas por la posible falta de homogeneidades del viento.

Varios resultados parciales del trabajo de esta tesis han sido reunidos en dos artículos publicados y en un tercero, que se encuentra en preparación. Estos artículos son:

1. Puls, Urbaneja, Venero, Repolust, Springmann, Jokuthy, \& Mokiem (2005),

2. Venero, Curé, Cidale, \& Araya (2016b),

3. Venero, Curé, Cidale, Puls, Haucke, Arcos, \& Araya (2017b).

Algunos resultados preliminares fueron presentados además en diversos congresos, en colaboración con otros autores:

- Venero \& Puls (2002),

- Haucke, Cidale, Venero, Cochetti, Torres, Arcos, \& Curé 2012),

- Haucke, Araya, Arcos, Curé, Cidale, Kanaan, Venero, \& Kraus (2015),

- Venero, Curé, \& Cidale (2016a),

- Venero, Cidale, Curé, \& Haucke (2017a),

- Cidale, Venero, Curé, \& Haucke (2017). 


\section{Capítulo 2}

\section{La Teoría de Vientos Impulsados por Radiación}

Resumen del capítulo: Se presentan las nociones básicas para el desarrollo de la teoría de vientos impulsados por radiación, en particular, la parametrización de la fuerza de radiación en líneas espectrales. Se resumen algunos de los logros más importantes de la teoría, en contraposición con las discrepancias que aún subsisten entre los modelos y las observaciones, teniendo en cuenta la gran diversidad de características de las supergigantes B. En el caso en que el lector se encuentre familiarizado con la teoría de vientos impulsados por radiación y desee acceder directamente al trabajo desarrollado en esta tesis, se le sugiere dirigir la lectura al capítulo 5, específicamente, comenzando en la sección 5.3 y siguientes.

\subsection{Idea conceptual}

La teoría de vientos impulsados por radiación propone que la transferencia de momento del campo de radiación estelar a las partículas que forman su atmósfera extendida (fotosfera y viento), es la responsable de acelerar al viento estelar. La transferencia de momento tiene lugar cuando un fotón incidente de frecuencia $\nu_{i n c}$, que proviene de la fotosfera estelar con una dirección $\theta_{i n c}$ respecto a la dirección radial, es absorbido por un ion. Luego, el fotón es reemitido de manera más o menos isotrópica con dirección de salida $\theta_{\text {sal }}$, también con respecto a la dirección radial. Este cambio de dirección en el ángulo $\theta$ sumado al cambio de frecuencia debido al efecto Doppler por el movimiento del ion, son los responsables de una transferencia neta de momento $\Delta P$ del campo de radiación al ion:

$$
\Delta P=h\left(\nu_{\text {inc }} \cos \theta_{\text {inc }}-\nu_{\text {sal }} \cos \theta_{\text {sal }}\right) / c
$$

Cuando se suman los cambios de momento correspondientes a todos los procesos de dispersión, las pérdidas y las ganancias debidas a los procesos de reemisión en la dirección no radial se cancelan debido a la simetría isotrópica, quedando activos solamente, la suma de los procesos dirigidos radialmente. Esto produce una aceleración radiativa neta en los iones, que se transmite a la totalidad del plasma que forma el viento, siempre que el momento ganado sea compartido, vía colisiones coulombianas, con los iones más abundantes de $\mathrm{H}$ y He. 
A pesar de que el $\mathrm{H}$ y el He son muy abundantes, estos elementos tienen pocas líneas en las regiones espectrales correspondientes al máximo de flujo (UV) de las estrellas tempranas. Por esta razón, el $\mathrm{H}$ y el He no son los absorbedores más eficientes de momento. En cambio, los iones de elementos pesados tales como C, N, O, Si, Mg y particularmente, el grupo del Fe, cuentan con un enorme número de líneas espectrales en el UV, por lo cual pueden absorber cantidades importantes de impulso del campo de radiación.

La transferencia de momento de iones de metales a las especies dominantes se realiza por colisiones o interacciones coulombianas (Castor et al. 1976). Esto se satisface con facilidad en vientos muy densos y se los modela con una aproximación de fluido único. Esta aproximación es válida siempre que el tiempo de escala en el cual los iones activos transfieren el momento a los iones pasivos sea pequeño, en comparación con el tiempo que necesitan los iones activos para escapar de su influencia. El acoplamiento de las especies químicas es óptimo en los vientos de las estrellas $\mathrm{O}$ y WR, pero para vientos de baja densidad como los que surgen alrededor de las estrellas B y A, puede haber un desacople entre los iones que absorben momento y el resto del plasma, lo que lleva a calentamiento friccional y altas temperaturas (Springmann \& Pauldrach 1992) o a vientos con múltiples componentes (Krtička \& Kubát|2001). Este último caso, ocurre una fuga de iones (ion runaway), la cual puede producir pequeñas tasas de pérdida de masa y grandes velocidades términales. En esta tesis no se considerarán estos casos extremos.

\subsubsection{Hipótesis de la teoría estándar}

Los vientos estelares impulsados por la presión de radiación se obtienen como solución de las ecuaciones de conservación de masa, de momento y de energía aplicadas al flujo de materia alrededor de las estrellas. Para resolver estas ecuaciones, es necesario hacer uso de aproximaciones que simplifican los modelos. La teoría básica de vientos impulsados por radiación está desarrollada bajo algunas hipótesis generales que pueden relajarse al incorporar nuevos fenómenos como, por ejemplo, la rotación estelar. En esta sección se resumen las aproximaciones básicas de esta teoría para, más adelante, indicar cuáles de estas suposiciones son adoptadas o no en esta tesis.

Una de las principales hipótesis de la teoría consiste en suponer que los vientos estelares son sistemas estacionarios, cuyas características ya se han estabilizado en un régimen de velocidades y una estructura de densidad que no cambian con el tiempo. Esta suposición no es completamente correcta ya que hay muchas evidencias de la variación espectral y fotométrica temporal que presentan las supergigantes B (ver por ejemplo, la sección 1.2.1 o algunos trabajos como los de Bresolin \& Kudritzki 2004. Clark et al. 2010; Chesneau et al. 2014, Kraus et al. 2015a). También, en contra de esta hipótesis, se puede citar la presencia de Componentes Discretas en Absorción (DACs, por su nombre en inglés: Discrete Absorption Components), que son componentes en absorción que se superponen a los perfiles de algunas líneas espectrales y que migran a través del perfil hacia el azul (sección 2.6.6). Sin embargo, se debe enfatizar que las propiedades básicas de los vientos, tales como las velocidades terminales y las tasas de pérdida de masa, pueden ser predichas en valores medios con aceptable precisión con la aproximación estacionaria (Puls et al. 2008b). 
También la teoría estándar supone que el viento es homogéneo y que puede ser representado con una estructura de densidad y de velocidades de variación suave, adoptando, ademas una composición química constante a través del viento. Esta hipótesis se ha puesto fuertemente en discusión en la última década por el problema del clumping, como fue mencionado en la sección 1.2.5, sobre el cual se ha dedicado considerables esfuerzos (por ejemplo, Puls et al. 2008b; Prinja \& Massa 2010; Šurlan et al. 2013 . Sundqvist et al. 2014).

El problema del clumping se vincula estrechamente con el hecho de que los vientos impulsados por radiación son intrínsecamente inestables, por lo que se prevé la formación de pequeñas perturbaciones a lo largo de su extensión, tanto en densidad como en velocidad (sección 2.6.1). Esta condición genera apartamientos a la hipótesis de estacionareidad y homogeneidad del viento. Estas perturbaciones no están incluidas en la teoría estándar.

Otra de las hipótesis importante concierne a la geometría de los vientos. La mayoría de los modelos son considerados usando simetría esférica, es decir, se trata de modelos 1-D cuya única variable es la distancia $r$ al centro del sistema. Resulta claro que hay firme evidencia de que muchas estrellas tempranas tienen severos apartamientos de esta geometría. Particularmente, las supergigantes B[e] transitan una fase en la cual eyectan grandes cantidades de material, el que se acumula en una estructura circumestelar discoidal, detectables a través de las emisiones de polvo y de moléculas como el CO y el SiO, y moviéndose en órbitas keplerianas (Kraus et al. 2015b). También pueden apreciarse estructuras asimétricas en nebulosas con forma de "reloj de arena" (hourglass) en supergigantes B como es el caso de Sher 25 (Hendry et al. 2008, Taylor et al. 2014), observable en la figura 1.6. Estas evidencias hace presuponer que la eyección de materia no sigue patrones con simetría esférica. A pesar de estos casos que comprenden objetos de subclases muy específicas entre las estrellas tempranas y, probablemente, la mayoría sean etapas transitorias en la evolución de las mismas, en general, las estrellas pueden ser consideradas como esferas y al viento como un flujo de materia en expansión radial, como una aceptable primera aproximación.

En el modelo estándar, es usual la adopción de una ley de velocidades analítica simple llamada ley $\beta$ de velocidades en lugar de la solución completa de las ecuaciones hidrodinámicas. Históricamente, una de los primeros trabajos en proponer la forma funcional general de la ley fue hecha por Lamers \& Rogerson (1978), la que se usó extensamente por ser muy apropiada para describir las características observadas en los vientos estelares de estrellas supergigantes O y B tempranas. Su justificación teórica se obtuvo en el trabajo de Castor, Abbott, \& Klein (1975), como se ve en la sección 2.2.10, que encontraron a esta forma para la ley de velocidades, como solución analítica hidrodinámica de los vientos impulsados por radiación, con un valor del parámetro $\beta$ de 0.5 .

La ley $\beta$ de velocidades tiene la siguiente expresión:

$$
v(r)=v_{\infty}\left(1-b \frac{R_{*}}{r}\right)^{\beta}, \quad b=1-\left(\frac{v\left(R_{*}\right)}{v_{\infty}}\right)^{\frac{1}{\beta}},
$$

donde $R_{*}$ es el radio de la estrella. La figura 2.1 muestra la forma de esta ley de velocidades para diferentes valores del parámetro $\beta$.

La constante $b$ fija en $v\left(R_{*}\right)$ al valor inicial de la velocidad en el borde interno del 


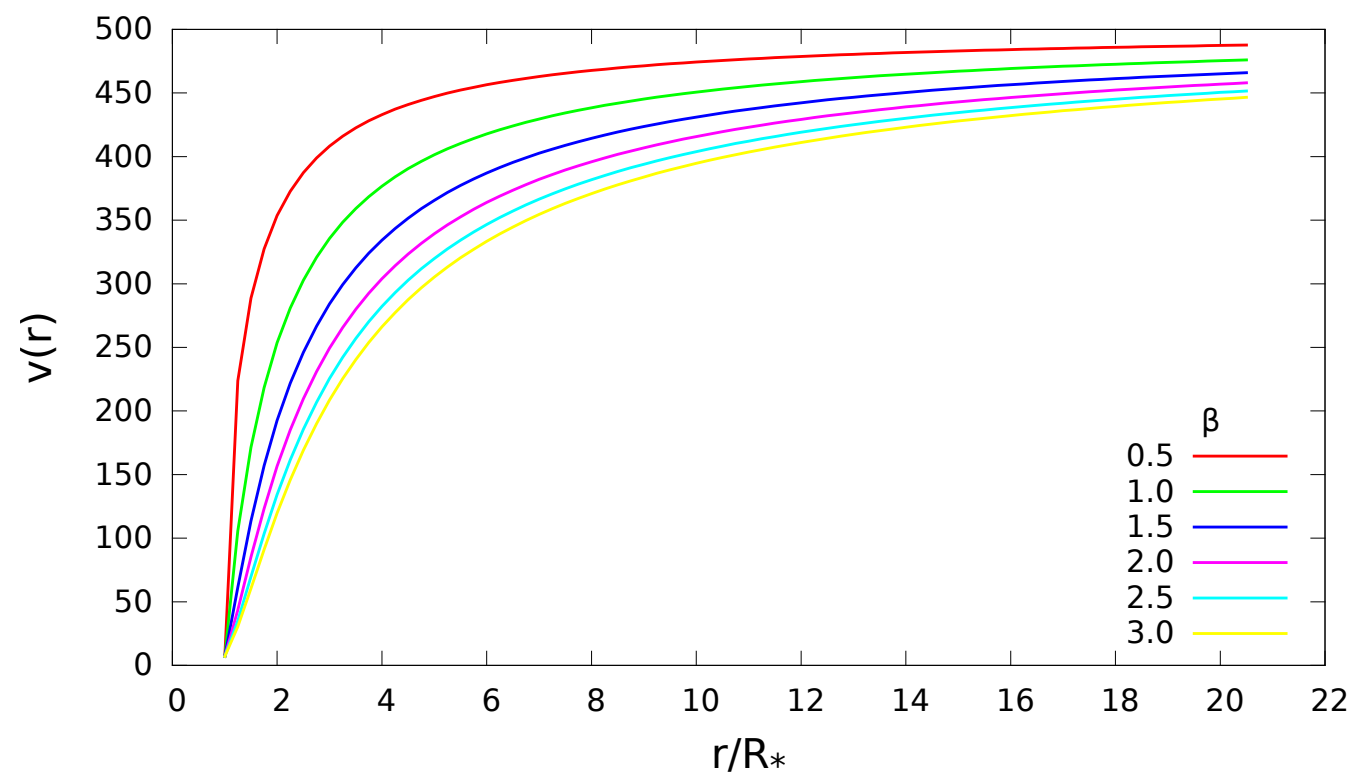

Figura 2.1. Ejemplos de la forma funcional de la ley $\beta$ de velocidades, para una velocidad terminal $\mathrm{v}_{\infty}$ de $500 \mathrm{~km} \mathrm{~s}^{-1}$ y una velocidad inicial de $0.1 \mathrm{~km} \mathrm{~s}^{-1}$. Las velocidades están expresadas en unidades de $\mathrm{km} \mathrm{s}^{-1}$.

viento que, en general, se lo considera del orden de la velocidad isotérmica del sonido $a$ (ecuación 2.15), en esa región. Normalmente, la especificación de ese valor no incide demasiado en los cálculos teóricos del espectro. En general, $v_{\infty}$ como $\beta$ son parámetros de ajuste a ser determinados a partir de mediciones en el espectro estelar (ver sección 2.2 .9 .

El parámetro $\beta$ da una estimación de cuán progresiva se da la aceleración en el viento. A mayor valor de $\beta$, más lejano es el punto en el cual se alcanza $v_{\infty}$. Para las estrellas O y B tempranas sus valores están comprendidos, aproximadamente, en el rango que va desde 0.7 a 1.5 (Puls et al. 1996; Kudritzki \& Puls 2000). En el caso de estrellas supergigantes de estos tipos espectrales, $\beta$ toma valores considerablemente mayores a los de ese rango (Lefever et al. 2007; Markova \& Puls 2008). Por su parte, las estrellas supergigantes A se modelan con las valores aún más altos: $\beta=3 \ldots 4$ (Stahl et al. 1991). Cabe mencionar que no hay una justificación teórica para estos valores tan altos adoptados para el parámetro $\beta$.

En la teoría estándar tampoco se consideran las fuerzas viscosas y por consiguiente la deposición de energía y momento por estos factores, como así tampoco la rotación estelar. De la misma manera, se prescinde de la presencia de campos eléctricos o magnéticos, y de la posible binariedad de las estrellas.

\subsection{Ecuaciones hidrodinámicas básicas}

La primera de las ecuaciones básicas que modelan un viento estelar es la ecuación de conservación de masa: 


$$
\frac{\partial \rho}{\partial t}+\nabla \cdot(\rho \boldsymbol{v})=0
$$

donde $\rho$ es la densidad y $\boldsymbol{v}$ es el vector velocidad 1 ambas cantidades dependientes de la posición y del tiempo. Esta expresión es válida para un elemento de volumen $d V$. Si se integra esta ecuación sobre el volumen $V$ contenido en una esfera de radio $r$ de la cual $d V$ es un elemento de volumen, la ecuación 2.3 queda de la forma:

$$
\frac{\partial}{\partial t} \int_{V} \rho d V+\int_{V} \boldsymbol{\nabla} \cdot(\rho \boldsymbol{v}) d V=0 .
$$

La integral de la densidad sobre el volumen es equivalente a la masa $M_{*}$ de la estrella. Por lo tanto, el primer término del miembro izquierdo de la ecuación 2.4 es la variación en el tiempo de la masa estelar, que puede expresarse como una derivada total de la forma:

$$
\frac{\partial}{\partial t} \int_{V} \rho d V \equiv-\frac{d M_{*}}{d t}=\dot{M}
$$

la cual es una derivada negativa porque se supone que la masa contenida en el volumen está disminuyendo. La cantidad $\dot{M}$ es la tasa de pérdida de masa definida en la sección 1.2.4.

En el segundo término del miembro izquierdo de la ecuación 2.4 puede usarse el teorema de la divergencia $\int_{S} \boldsymbol{F} \cdot \breve{\boldsymbol{n}} d S=\int_{V} \boldsymbol{\nabla} \cdot \boldsymbol{F} d V$ (donde $d S$ es un elemento de superficie $S$ que rodea al volumen $V, \boldsymbol{F}$ es un campo vectorial dado y $\breve{\boldsymbol{n}}$ es un versor en la dirección perpendicular a la superficie), transformando la integral sobre el volumen en una integral del flujo de materia que atraviesa la superficie. Si se adopta la simetría esférica, es decir, si las variables sólo dependen de la coordenada radial $r$, el flujo de materia $\rho \boldsymbol{v}$ es idéntico para cada elemento de superficie. Si, además, se supone que el medio es homogéneo, la integral se transforma como:

$$
\int_{V} \boldsymbol{\nabla} \cdot(\rho \boldsymbol{v}) d V=\int_{S}(\rho \boldsymbol{v}) \cdot \breve{\boldsymbol{r}} d S=4 \pi r^{2} \rho v .
$$

donde $v=\boldsymbol{v} \cdot \breve{\boldsymbol{r}}$ (con $\breve{\boldsymbol{r}}$, el versor radial), es la proyección en la dirección radial del vector velocidad.

Con estas consideraciones, la ecuación 2.3 queda como:

$$
\dot{M}=-\frac{d M_{*}}{d t}=4 \pi r^{2} \rho v=\text { constante, }
$$

que es la expresión integral de la ecuación de continuidad, en simetría esférica.

La segunda ecuación a considerar es la ecuación de conservación del momento lineal o ecuación de Navier-Stokes (Landau \& Lifshitz 1987):

$$
\frac{\partial \boldsymbol{v}}{\partial t}+(\boldsymbol{v} \cdot \boldsymbol{\nabla}) \boldsymbol{v}=-\frac{1}{\rho} \boldsymbol{\nabla} P+\boldsymbol{g}+\frac{1}{\rho}\left[\eta \boldsymbol{\nabla}^{2} \boldsymbol{v}+\left(\zeta+\frac{1}{3} \eta\right) \boldsymbol{\nabla}(\boldsymbol{\nabla} \cdot \boldsymbol{v})\right],
$$

\footnotetext{
${ }^{1}$ Nota: En las fórmulas, las cantidades expresadas en negrita denotan vectores.
} 
cuyo miembro izquierdo corresponde a la aceleración de un elemento de volumen $D \boldsymbol{v} / D t$ (expresada como derivada lagrangiana) y está constituido por dos términos. El primer término es la derivada $\partial \boldsymbol{v} / \partial t$, que representa el cambio de velocidad que ocurre en el elemento de volumen durante un $d t$ en un punto fijo del espacio.

El segundo término del miembro izquierdo, corresponde a la diferencia entre las velocidades (para un mismo instante de tiempo) que existe entre dos puntos separados en una distancia $d \boldsymbol{r}$, entre ambos extremos del elemento de volumen elegido. Usando un sistema de coordenadas $(\mathrm{x}, \mathrm{y}, \mathrm{z})$ auxiliar, este término puede expresarse como:

$$
\frac{d x}{d t} \frac{\partial \boldsymbol{v}}{\partial x}+\frac{d y}{d t} \frac{\partial \boldsymbol{v}}{\partial y}+\frac{d z}{d t} \frac{\partial \boldsymbol{v}}{\partial z}=\left(\frac{d \boldsymbol{r}}{d t} \cdot \nabla\right) \boldsymbol{v}=(\boldsymbol{v} \cdot \boldsymbol{\nabla}) \boldsymbol{v}
$$

como aparece en la ecuación 2.8 .

El lado derecho de la ecuación 2.8 contiene la sumatoria de fuerzas por unidad de masa que producen la aceleración del elemento de gas. El primer término representa el gradiente de presión del gas $P$. El segundo término corresponde a las fuerzas externas tales como la gravitación o las fuerzas radiativas, reunidas en el vector $\boldsymbol{g}$. El último término corresponde al frenado por la viscosidad del gas, caracterizado por los coeficientes de viscosidad $\eta$ y $\zeta$ (ecuación 15.6 de Landau \& Lifshitz 1987), los cuales son despreciables en los modelos de vientos estelares porque las densidades son muy bajas. Por lo tanto, la ecuación 2.8 se simplifica a la expresión:

$$
\frac{\partial \boldsymbol{v}}{\partial t}+(\boldsymbol{v} \cdot \boldsymbol{\nabla}) \boldsymbol{v}=-\frac{1}{\rho} \boldsymbol{\nabla} P+\boldsymbol{g}
$$

En un sistema de coordenadas esféricas, en el cual el vector velocidad es $\boldsymbol{v}=$ $\left(v_{r}, v_{\theta}, v_{\phi}\right)$, la ecuación vectorial 2.10 contiene tres componentes:

$$
\begin{gathered}
\frac{\partial v_{r}}{\partial t}+v_{r} \frac{\partial v_{r}}{\partial r}+\frac{v_{\theta}}{r} \frac{\partial v_{r}}{\partial \theta}+\frac{v_{\phi}}{r \sin \theta} \frac{\partial v_{r}}{\partial \phi}-\frac{1}{r}\left(v_{\theta}^{2}+v_{\phi}^{2}\right)=-\frac{1}{\rho} \frac{\partial P}{\partial r}+g_{r} \\
\frac{\partial v_{\theta}}{\partial t}+v_{r} \frac{\partial v_{\theta}}{\partial r}+\frac{v_{\theta}}{r} \frac{\partial v_{\theta}}{\partial \theta}+\frac{v_{\phi}}{r \sin \theta} \frac{\partial v_{\theta}}{\partial \phi}+\frac{1}{r}\left(v_{r} v_{\theta}-v_{\phi}^{2} \cot \theta\right)=-\frac{1}{\rho r} \frac{\partial P}{\partial \theta}+g_{\theta} \\
\frac{\partial v_{\phi}}{\partial t}+v_{r} \frac{\partial v_{\phi}}{\partial r}+\frac{v_{\theta}}{r} \frac{\partial v_{\phi}}{\partial \theta}+\frac{v_{\phi}}{r \sin \theta} \frac{\partial v_{\phi}}{\partial \phi}+\frac{v_{\phi}}{r}\left(v_{r}+v_{\theta} \cot \theta\right)=-\frac{1}{\rho r \sin \theta} \frac{\partial P}{\partial \phi}+g_{\phi},
\end{gathered}
$$

donde el vector de las fuerzas externas tiene como componentes: $\boldsymbol{g}=\left(g_{r}, g_{\theta}, g_{\phi}\right)$.

En un sistema estacionario, en cada punto del espacio habrá siempre la misma velocidad, por lo cual el término $\partial \boldsymbol{v} / \partial t$ de la ecuación 2.10 se anula. Esta simplificación corresponde a anular el primer término del miembro izquierdo de cada una de las ecuaciones 2.11.

Si además se considera que el sistema tiene simetría esférica, el vector velocidad estará representado como $\boldsymbol{v}=\left(v_{r}, 0,0\right)$. Por simplicidad, se omitirá el subíndice $r$ y se llamará directamente $v$ a la componente radial de la velocidad. Por otro lado, sólo se consideran fuerzas actuando en la dirección radial, de modo que el vector de fuerzas será $\boldsymbol{g}=\left(g_{r}, 0,0\right)$.

En estas condiciones sólo queda la primera ecuación del sistema 2.11, con la forma simplificada: 


$$
v \frac{\partial v}{\partial r}=-\frac{1}{\rho} \frac{\partial P}{\partial r}+g_{r}
$$

donde $v$ y $g_{r}$ son las componentes radiales del vector velocidad y de las fuerzas externas, respectivamente. Para las fuerzas se considera, aquí, la suma de:

$$
g_{r}=-\frac{G M_{*}}{r^{2}}+g_{\text {rad }}^{C}+g_{\text {rad }}^{L}
$$

siendo el primer término, la fuerza gravitatoria y los dos siguientes, la fuerza producida por la transferencia de momento a través de la radiación del continuo y de líneas, respectivamente.

Considerando que el fluido se comporta como un gas ideal, puede reemplazarse la presión P por la ecuación de estado:

$$
P=N k_{B} T=\frac{\rho k_{B} T}{\mu m_{H}}=\rho a^{2} .
$$

donde $\mathrm{N}$ es la densidad numérica de partículas, $k_{B}$ es la constante de Boltzmann y $m_{H}$ es la masa del átomo de hidrógeno. En la última igualdad de esta ecuación se introduce la velocidad isoterma del sonido $a$ :

$$
a=\sqrt{\frac{k_{B} T}{\mu m_{H}}},
$$

que, aunque depende de la temperatura, se la considera constante (calculada a partir de un valor de referencia de $T$ ). Esta simplificación se basa en que, como los gradientes de temperatura de los vientos son suaves, se los modela como sistemas isotermos. En la ecuación 2.14, $\mu$ es el peso molecular medio de las partículas de gas, que representa el número de partículas por nucleón que incorpora cada especie que aporta masa. Esta cantidad se considera constante para una dada composición química. Por ejemplo, si el medio está formado por hidrógeno puro neutro, se aporta una sola partícula para un sólo nucleón, entonces $\mu=1 / 1=1$. Si el medio está formado por hidrógeno puro ionizado, se aportan dos partículas $\left(p^{+}\right.$y $\left.e^{-}\right)$, entonces $\mu=1 / 2$. Si el medio está formado por helio puro ionizado, se tendrán tres partículas (núcleo y dos $e^{-}$) para $4 m_{H}$ (nucleón), entonces $\mu=3 / 4$. Para metales ionizados, el número de nucleones es dos veces el número $Z$, entonces $1 / \mu=(Z+1) /(2 Z)$. En general, para una mezcla, se cumple que $1 / \mu=\sum_{i} X_{i} / \mu_{i}$, donde a cada especie $i$ le corresponde una fracción de masa $X_{i}$. Entonces para una mezcla completamente ionizada, $\mu$ puede ser aproximado como:

$$
\frac{1}{\mu}=2 X+\frac{3}{4} Y+\frac{1}{2} Z
$$

donde $\mathrm{X}, \mathrm{Y}$ y Z son las fracciones de masa del hidrógeno, del helio y de los metales, respectivamente, de modo que $X+Y+Z=1$. En el caso de composición solar, $X=0.73, Y=0.24$ y $Z=0.03$, de modo que $\mu=0.604$.

Reemplazando $\rho$ de la ecuación 2.3, y expresando la ecuación 2.12 con derivadas totales, se obtiene: 


$$
\left(v-\frac{a^{2}}{v}\right) \frac{d v}{d r}=\frac{2 a^{2}}{r}-\frac{d a^{2}}{d r}+g_{r}
$$

donde

$$
g_{\text {rad }}=g_{\text {rad }}^{C}+g_{\text {rad }}^{L},
$$

es decir, la fuerza de radiación es la suma de las contribuciones de la aceleración producida por la radiación en el continuo y en las líneas espectrales. En la ecuación 2.17, el término $-d a^{2} / d r$ es aproximadamente cero, ya que vientos son considerados casi isotermos.

La especificación de la velocidad y la densidad para cada punto a lo largo del viento desde la superficie estelar (es decir, la ley de velocidades y la ley de densidades, respectivamente), da una descripción hidrodinámica completa del viento en un sistema $1 \mathrm{D}$.

Finalmente, la tercera ecuación a considerar es la ecuación de conservación de la energía, que puede expresarse como:

$$
\frac{\partial P}{\partial t}+\boldsymbol{v} \cdot \nabla P-\left(\frac{\gamma P}{\rho}\right)\left[\frac{\partial \rho}{\partial t}+\boldsymbol{v} \cdot \nabla \rho\right]=H(T),
$$

donde $P$ es la presión del gas y la constante $\gamma$ es el cociente de los calores específi$\cos C_{p} / C_{v}$, que vale $5 / 3$ para un gas ideal monoatómico. Esta ecuación que establece la conservación de la energía interna, siendo $H(T)$ la tasa neta de calentamiento o enfriamiento, la cual es dependiente de la temperatura $T$. En los vientos de estrellas tempranas, los términos de calentamiento y enfriamiento radiativo en $H(T)$ dominan esta ecuación, y las escalas de tiempo para las cuales el gas gana o pierde energía son cortos en comparación con las escalas de tiempo del flujo.

La ecuación 2.19 establece una ley de temperaturas $T(r)$ a lo largo del viento, siendo la condición de equilibrio radiativo, una de sus formas más frecuentemente adoptada. Drew (1989) mostró que, para el caso de equilibrio radiativo, la temperatura decae lentamente con el radio, desde un valor de $\sim 0.8 \mathrm{~T}_{\text {eff }}$ en la superficie estelar, hasta $\sim 0.6 \mathrm{~T}_{\text {eff }}$, a una distancia del doble del radio estelar. Por esta razón, es común que se adopte, directamente, una ley de temperaturas constante, con un valor de alrededor de $0.75 \mathrm{~T}_{\text {eff }}$ para todo el viento.

\subsubsection{La aceleración debida al continuo de radiación}

Para documentar las aproximaciones usadas en la teoría estándar de vientos impulsados por radiación para obtener una expresión para la aceleración total debida a la radiación, se siguen los lineamientos planteados por Cranmer (1996).

La aceleración $g_{\text {rad }}^{C}$ que aparece en la ecuación 2.18, se debe al continuo del campo de radiación en un punto $\boldsymbol{r}$. Se trata de una de las vías para transferir momento del campo de radiación al fluido por medio de la absorción y la dispersión (scattering) de fotones del continuo por parte de electrones e iones. La expresión más general de esta aceleración es: 


$$
\boldsymbol{g}_{\text {rad }}^{C}(\boldsymbol{r})=\frac{1}{c} \oint \int_{\nu=0}^{\infty} \kappa_{C} I_{\nu}(\boldsymbol{r}, \breve{\boldsymbol{n}}) \breve{\boldsymbol{n}} d \omega d \nu
$$

donde $\kappa_{C}$ es el coeficiente de extinción total (absorción más dispersión) de la radiación de continuo. El versor $\breve{\boldsymbol{n}}$ indica la dirección del flujo de campo de radiación, el cual es integrado para ángulos sólidos $\omega$ que rodean esta dirección.

En las estrellas tempranas, la contribución a la transferencia de momento debido a la dispersión de la radiación por los electrones libres es mucho mayor que la generada en las transiciones libre-libre o las ligado-libre. Por lo tanto, se considera $\kappa_{C}=\sigma_{e}$ como la opacidad por dispersión Thomson, despreciando las otras contribuciones mencionadas. Considerando que $\sigma_{e}$ es practicamente constante, la ecuación 2.20 se simplifica como:

$$
\boldsymbol{g}_{r a d}^{C}=\frac{\sigma_{e}}{c} \oint \int_{\nu=0}^{\infty} I_{\nu}(\boldsymbol{r}, \breve{\boldsymbol{n}}) \breve{\boldsymbol{n}} d \omega d \nu
$$

La integral doble de esta ecuación equivale al primer momento del campo de radiación, es decir, el flujo, y al ser integrada a lo largo del espectro, es el flujo bolométrico. Este flujo, en simetría esférica puede escribirse como:

$$
\mathcal{F}_{*}=\frac{L_{*}}{4 \pi r^{2}}
$$

donde $L_{*}$ es la luminosidad bolométrica de la estrella y $r$ la distancia al centro estelar.

De esta manera, la aceleración debida a la absorción de radiación del continuo es:

$$
g_{r a d}^{C}=\frac{\sigma_{e} L_{*}}{4 \pi r^{2} c}
$$

La dependencia con $r^{-2}$ de la última ecuación permite unir este término con el correspondiente a la aceleración de la gravedad:

$$
g+g_{\text {rad }}^{C}=-\frac{G M_{*}(1-\Gamma)}{r^{2}}
$$

donde $\Gamma$ es:

$$
\Gamma=\frac{\sigma_{e} L_{*}}{4 \pi c G M_{*}}=\frac{L_{*}}{L_{E d d}}
$$

siendo $L_{E d d}$ la luminosidad de Eddington, que es el valor máximo de la luminosidad que equilibra exactamente la gravedad de la estrella, manteniendo la condición de equilibrio hidrostático.

\subsubsection{La aceleración debida a la absorción de momento en las líneas espectrales}

La aceleración debida a la contribución de numerosas líneas espectrales individuales es:

$$
\boldsymbol{g}_{\text {rad }}^{L}(\boldsymbol{r})=\sum_{\text {lineas }} \frac{1}{c} \oint \int_{\nu=0}^{\infty} \bar{\kappa}_{L}(\nu) I_{\nu}(\boldsymbol{r}, \breve{\boldsymbol{n}}) \breve{\boldsymbol{n}} d \omega d \nu
$$


en la que $\bar{\kappa}_{L}(\nu)$ es el coeficiente de absorción en líneas, cuya dependencia con la frecuencia puede expresarse usando la función normalizada de perfil de línea $\phi\left(\nu-\nu^{\prime}\right)$, como:

$$
\bar{\kappa}_{L}(\nu)=\kappa_{L} \phi\left(\nu-\nu^{\prime}\right)
$$

Esto permite reescribir a la aceleración como:

$$
\boldsymbol{g}_{\text {rad }}^{L}(\boldsymbol{r})=\sum_{\text {lineas }} \frac{\kappa_{L}}{c} \oint \int_{\nu=0}^{\infty} \phi\left(\nu-\nu^{\prime}\right) I_{\nu}(\boldsymbol{r}, \breve{\boldsymbol{n}}) \breve{\boldsymbol{n}} d \omega d \nu
$$

y considerando que la frecuencia del fotón emitido por la estrella es $\nu_{0}$, los iones del flujo de materia lo recibirán con el desplazamiento Doppler:

$$
\nu^{\prime}=\nu_{0}\left[1+\frac{1}{c} \breve{\boldsymbol{n}} \cdot \boldsymbol{v}(\boldsymbol{r})\right] .
$$

Comúnmente se define la variable $x$, como el desplazamiento en frecuencia a partir del centro de la línea, en unidades Doppler:

$$
x=\left(\frac{\nu-\nu_{0}}{\Delta \nu_{D}}\right)
$$

donde se define el ancho Doppler como:

$$
\Delta \nu_{D}=\nu_{0} v_{t h} / c
$$

con $v_{t h}$, la velocidad térmica, es decir, la velocidad media de los iones que conducen al flujo de gas. Esta velocidad puede calcularse como:

$$
v_{t h}=\sqrt{\frac{2 k_{B} T}{A_{i} m_{H}}}
$$

en la que $A_{i}$ es la número másico (cantidad de nucleones) de los iones. La $v_{t h}$ puede compararse con la velocidad isoterma del sonido $a$, definida en la ecuación 2.15 haciendo:

$$
\frac{v_{t h}}{a}=\sqrt{\frac{2 \mu_{i}}{A_{i}}}=\sqrt{\frac{Z_{i}+1}{Z_{i} A_{i}}},
$$

siendo la última igualdad, para valores de $\mu$ correspondientes a iones pesados, tales como el $\mathrm{C}$, el $\mathrm{N}$ y el $\mathrm{O}$. En el caso del $\mathrm{O}, Z=8$ y $A=16$, la ecuación 2.33 da una relación $v_{t h} / a$ de 0.265 . Algunas veces, se usa el valor de $v_{t h}$ calculado directamente para el átomo de $\mathrm{H}$, como una medida de calibración, para el cual $v_{t h} / a \gtrsim 1$.

Con estas expresiones, la aceleración debida a la absorción de momento en líneas (ecuación 2.28) queda:

$$
\boldsymbol{g}_{\text {rad }}^{L}(\boldsymbol{r})=\sum_{\text {lineas }} \frac{\kappa_{L} \Delta \nu_{D}}{c} \oint \int_{x=-\infty}^{\infty} \phi\left(x-\frac{\breve{\boldsymbol{n}} \cdot \boldsymbol{v}(\boldsymbol{r})}{v_{t h}}\right) I_{\nu}(\boldsymbol{r}, \breve{\boldsymbol{n}}) \breve{\boldsymbol{n}} d \omega d x
$$


en la cual se ha extendido intencionalmente el límite inferior de integración en $x$ a valores negativos, considerando que la función perfil es una función angosta y acotada de la frecuencia.

Para hacer más simple la ecuación 2.34, es necesario disponer de una forma explícita para la intensidad específica $I_{\nu}(\boldsymbol{r}, \breve{\boldsymbol{n}})$. La intensidad específica puede expresarse como solución formal de la ecuación de transporte radiativo en su forma general (Mihalas 1978):

$$
I_{\nu}\left(\tau_{\nu}\right)=\int_{0}^{\tau_{\nu}} S_{\nu}\left(\tau_{\nu}\right) e^{-\left(\tau_{\nu}-t_{\nu}\right)} d t_{\nu}+I_{\nu}^{c} e^{-\tau_{\nu}},
$$

en la que $S_{\nu}\left(\tau_{\nu}\right)$ es la función fuente de los fotones de la línea espectral creados en el viento. La intensidad específica $I_{\nu}^{c}$ representa el campo de radiación de la estrella que alcanza al punto en cuestión, caracterizado por $\tau_{\nu}$. La profundidad óptica de la línea $\tau_{\nu}$ para el medio en movimiento en una dirección arbitraria $l$, se define como:

$$
\tau_{\nu}=\int_{0}^{l} \kappa_{L} \rho\left(l^{\prime}\right) \phi\left(x-\frac{\breve{\boldsymbol{n}} \cdot \boldsymbol{v}(\boldsymbol{r})}{v_{t h}}\right) d l^{\prime},
$$

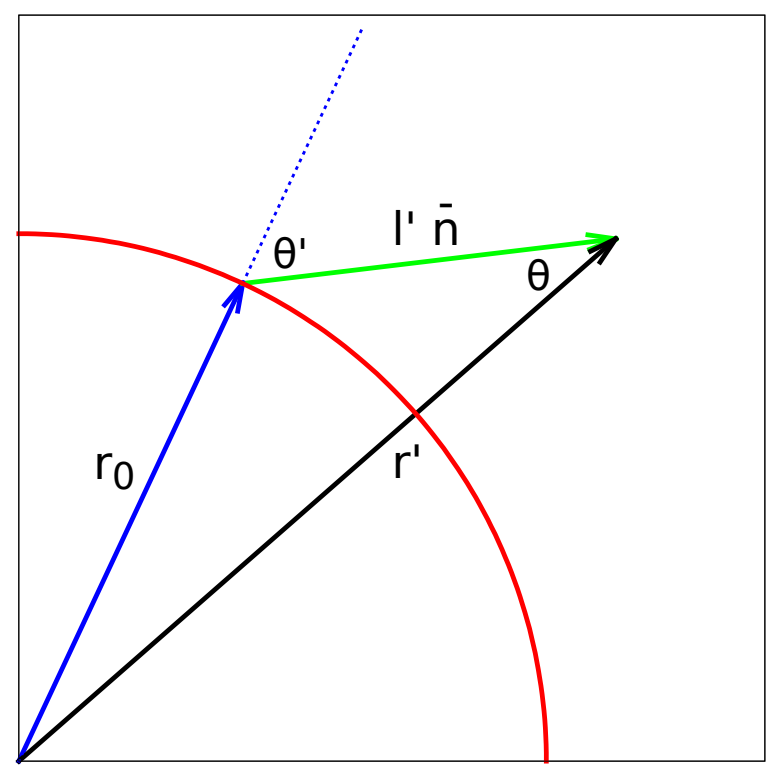

Figura 2.2. Referencia de los vectores y ángulos que se usan para ubicar al punto donde se calcula la aceleración radiativa en el viento. Más detalles en el texto.

La integral de esta última expresión se extiende a lo largo del camino $l^{\prime}$ en la dirección arbitraria $\breve{\boldsymbol{n}}$. Siendo $\boldsymbol{r}_{\mathbf{0}}$ la posición de la superficie estelar desde donde se emite el fotón $\left(l^{\prime}=0\right)$ y $\boldsymbol{r}^{\prime}$, el vector que señala el punto en el cual se está evaluando la aceleración (donde $l^{\prime}=l$ ). En esta suma vectorial, que se esquematiza en la figura 2.2, se cumple:

$$
\boldsymbol{r}^{\prime}=\boldsymbol{r}_{\mathbf{0}}+l^{\prime} \breve{\boldsymbol{n}}
$$


La intensidad específica expresada según la ecuación 2.35 debe reemplazarse en la expresión de la aceleración radiativa debido a las líneas (ecuación 2.34). Sin embargo, el término que corresponde a la integral de la función fuente de los fotones de línea creados en el viento resulta ser muy pequeño y, en principio, puede despreciarse. Esta contribución de radiación difusa de fotones producidos por dispersión o emisión a lo largo del viento está sistemáticamente desplazada al rojo por el movimiento monotonamente acelerado del viento y, por otro lado, la suma de sus componentes da una contribución simétrica en la dirección radial, la cual es considerablemente menor que la radiación proveniente de la fotosfera estelar. Esta contribución sólo se desprecia para el cálculo de la fuerza de radiación, pero no en el transporte de radiación, como se ve en la sección 5.1 .2 .

Se obtiene entonces:

$$
\boldsymbol{g}_{\text {rad }}^{L}(\boldsymbol{r})=\sum_{\text {lineas }} \frac{\kappa_{L} \Delta \nu_{D}}{c} \oint \int_{x=-\infty}^{\infty} \phi\left(x-\frac{\breve{\boldsymbol{n}} \cdot \boldsymbol{v}(\boldsymbol{r})}{v_{\text {th }}}\right) I_{\nu}^{c} e^{-\tau_{\nu}} \breve{\boldsymbol{n}} d \omega d x
$$

en la cual la profundidad óptica $\tau_{\nu}$ está dada por la expresión de la ecuación 2.36.

\subsubsection{La aproximación de Sobolev}

La integral en la definición de la profundidad óptica dada por la ecuación 2.36 es complicada y requiere de aproximaciones para ser resuelta. Sobolev (1957, 1960) introdujo una aproximación extensamente aplicada en la teoría de vientos impulsados por radiación. Esta aproximación, aplicada al transporte de radiación, es resumida en la sección 5.1.1 y puede consultarse, para más detalle, por ejemplo, en el trabajo de Rybicki \& Hummer (1978). En la aproximación de Sobolev se supone que, si las velocidades del fluido son suficientemente altas, la función perfil de línea domina la integral de la ecuación 2.36 a lo largo del camino $l^{\prime}$, limitando su valor a una región estrecha para la cual el rango de frecuencias coincide con la capacidad de absorción del ion, debido al corrimiento Doppler producido por el campo de velocidades. En ese caso, la opacidad $\kappa_{L}$ y la densidad $\rho\left(l^{\prime}\right)$ pueden ser considerados constantes y ser extraídas de la integral.

La condición para que la aproximación de Sobolev se verifique es que la frecuencia del fotón que es absorbido por un ion en el marco del observador cambie en un valor $\Delta \nu_{D}$, un ancho Doppler, cuando la velocidad del fluido se incrementa de $v$ a $v+v_{t h}$. De esta manera, en esta aproximación, la interacción entre los fotones y el material del viento se da en una región muy localizada, conocida como región de resonancia.

En fórmulas, la condición puede escribirse en términos de la llamada longitud de Sobolev $\left(L_{s o b}\right)$, la cual debe ser mucho menor que la escala de altura $H_{p}$ característica del medio. Es decir:

$$
L_{s o b} \approx \frac{v_{t h}}{d v / d r} \ll H_{p}=\frac{\rho}{d \rho / d r} \approx \frac{v}{d v / d r},
$$

la cual implica que, o bien $v_{t h}$ es muy pequeña comparada con las velocidades típicas del viento o que estas velocidades son supersónicas $\left(v \gg v_{t h}\right)$. Considerando que $v_{t h}=$ 
$0.3 a<a$ (siendo $a$ la velocidad del sonido isoterma, definida en la ecuación 2.15), aún en el punto sónico del viento $(v=a)$, la condición de Sobolev también se satisface.

Bajo la aproximación de Sobolev, la profundidad óptica dada por la ecuación 2.36 puede escribirse como:

$$
\tau_{\nu}(\boldsymbol{r})=\kappa_{L} \rho(\boldsymbol{r}) \int_{0}^{l} \phi\left(x-\frac{\breve{\boldsymbol{n}} \cdot \boldsymbol{v}\left(\boldsymbol{r}^{\prime}\right)}{v_{t h}}\right) d l^{\prime} .
$$

Considerando que el viento es monótonamente creciente, se puede hacer un cambio de variables:

$$
\begin{gathered}
x^{\prime}=x-\frac{\breve{\boldsymbol{n}} \cdot \boldsymbol{v}\left(\boldsymbol{r}^{\prime}\right)}{v_{t h}}, \\
d x^{\prime}=-\frac{1}{v_{t h}} d\left[\breve{\boldsymbol{n}} \cdot \boldsymbol{v}\left(\boldsymbol{r}^{\prime}\right)\right]=-\frac{1}{v_{t h}} \breve{\boldsymbol{n}} \cdot \nabla\left[\breve{\boldsymbol{n}} \cdot \boldsymbol{v}\left(\boldsymbol{r}^{\prime}\right)\right] d l^{\prime} .
\end{gathered}
$$

En la última igualdad se tuvo en cuenta que, de acuerdo a la ecuación 2.36, $d r^{\prime}=$ $\breve{\boldsymbol{n}} d l^{\prime}$ y que el vector $\breve{\boldsymbol{n}}$ es constante.

El factor $\breve{\boldsymbol{n}} \cdot \nabla\left[\breve{\boldsymbol{n}} \cdot \boldsymbol{v}\left(\boldsymbol{r}^{\prime}\right)\right]$ es muy importante porque contiene el gradiente de velocidades, que surge del cambio de variables. Para una dirección arbitraria $\breve{\boldsymbol{n}}$ que forma un ángulo $\theta$ con la dirección radial, en un flujo puramente radial, este factor toma la forma (ver la demostración en el apéndice .1):

$$
\breve{\boldsymbol{n}} \cdot \nabla\left[\breve{\boldsymbol{n}} \cdot \boldsymbol{v}\left(\boldsymbol{r}^{\prime}\right)\right]=\mu^{2} \frac{\partial v}{\partial r}+\left(1-\mu^{2}\right) \frac{v}{r},
$$

donde $\mu=\cos \theta$. Si se consideran direcciones radiales $(\mu=1)$, el factor se simplifica al gradiente puro de la velocidad radial $(\partial v / \partial r)$.

El gradiente de velocidades es una función lentamente variable en el camino de integración, por lo que este factor también puede extraerse de la integral de la ecuación 2.40, como se hizo para $\kappa_{L}$ y $\rho$. Por lo tanto, con el cambio de variables, la ecuación 2.40 toma la forma llamada profundidad óptica de Sobolev:

$$
\tau_{s o b}(x, \boldsymbol{r})=\tau_{0} \Phi(x, \boldsymbol{r})
$$

donde

$$
\tau_{0}=\kappa_{L} \rho\left(\frac{v_{t h}}{\breve{\boldsymbol{n}} \cdot \nabla\left[\breve{\boldsymbol{n}} \cdot \boldsymbol{v}\left(\boldsymbol{r}^{\prime}\right)\right]}\right)=\kappa_{L} \rho\left(\frac{v_{t h}}{\mu^{2} \frac{\partial v}{\partial r}+\left(1-\mu^{2}\right) \frac{v}{r}}\right)=\kappa_{L} \rho L_{s o b}
$$

que ofrece una definición más adecuada para la longitud de Sobolev $L_{\text {sob }}$, y

$$
\Phi(x, \boldsymbol{r}) \equiv \int_{x-\breve{n} \cdot \boldsymbol{v}(\boldsymbol{r}) / v_{t h}}^{\infty} \phi\left(x^{\prime}\right) d x^{\prime} .
$$

La función $\Phi(x, \boldsymbol{r})$ es proporcional a la función error para un perfil de tipo Doppler puro o es una función escalón, si el perfil es una delta de Dirac. El primer caso se ilustra en la figura 2.3 .

Por lo tanto, la ecuación 2.38 queda: 


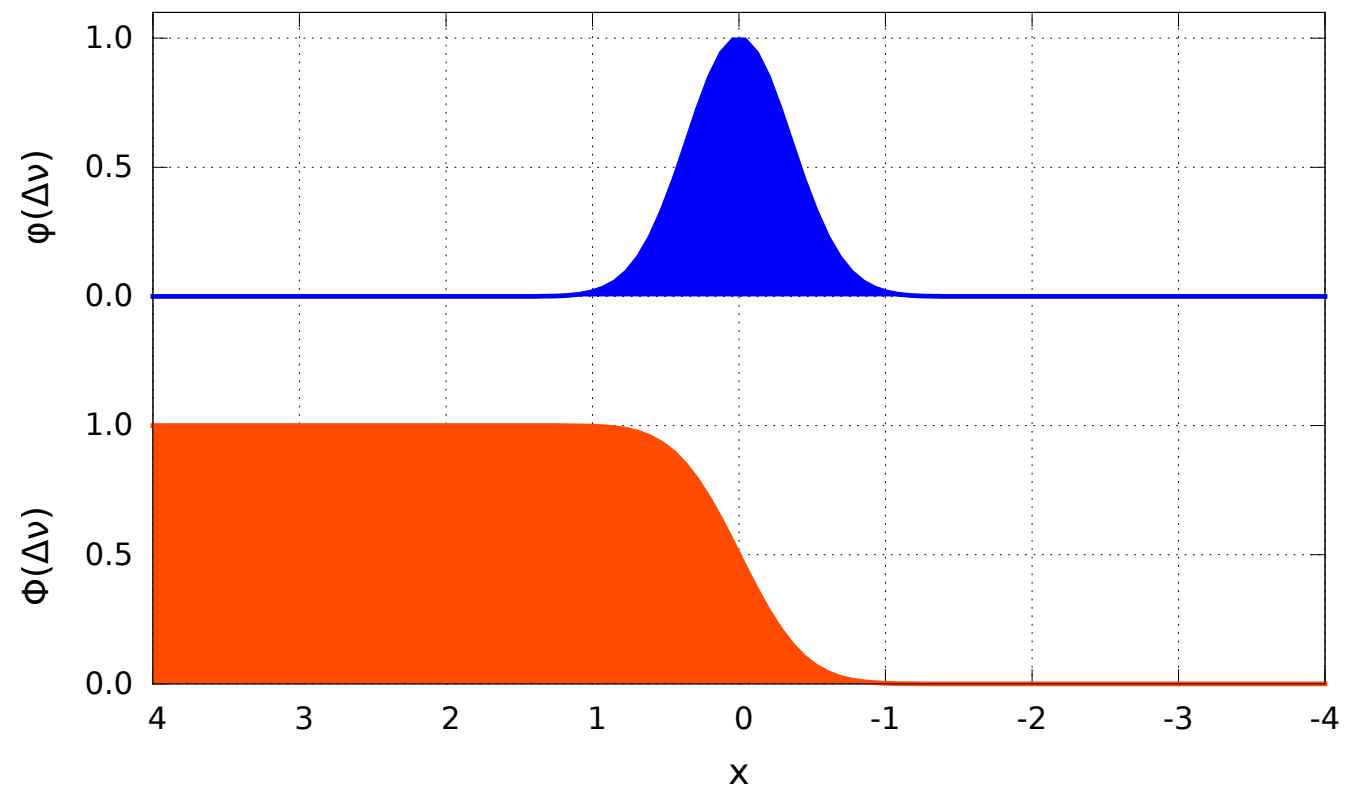

Figura 2.3. Para un perfil de línea $\phi(x)$ de tipo gaussiano (arriba), la correspondiente función $\Phi(x)$ es proporcional a una función error (debajo).

$$
\boldsymbol{g}_{\text {rad }}^{L}=\sum_{\text {lineas }} \frac{\kappa_{L} \Delta \nu_{D}}{c} \oint \int_{x=-\infty}^{\infty} \phi(x) I_{\nu}^{c} e^{-\tau_{0} \Phi(x, r)} \breve{\boldsymbol{n}} d \omega d x,
$$

en la cual $I_{\nu}^{c}$ puede extraerse de la integral en $x$ como $I^{c}(\omega)$, ya que es una función que varía muy lentamente con la frecuencia y el perfil $\phi(x)$ es muy delgado. Entonces se obtiene:

$$
\boldsymbol{g}_{\text {rad }}^{L}=\sum_{\text {lineas }} \frac{\kappa_{L} \Delta \nu_{D}}{c} \oint I^{c}(\omega) \breve{\boldsymbol{n}} d \omega \int_{x=-\infty}^{\infty} e^{-\tau_{0} \Phi(x, \boldsymbol{r})} d \Phi(x, \boldsymbol{r}),
$$

que puede resolverse obteniendo finalmente:

$$
\boldsymbol{g}_{\text {rad }}^{L}=\sum_{\text {lineas }} \frac{\kappa_{L} \nu_{0} v_{t h}}{c^{2}} \oint I^{c}(\omega) \breve{\boldsymbol{n}} d \omega\left[\frac{1-e^{-\tau_{0}}}{\tau_{0}}\right] .
$$

Para evaluar esta aceleración es necesario sumar sobre miles y millones de transiciones individuales que aporten momento a los iones que impulsan al viento.

\subsubsection{Aceleración radiativa para fuentes puntuales}

La expresión más simple para la aceleración radiativa surge si se supone que la fuente es puntual, es decir, que todos los fotones provenientes de la estrella son radiales. Esta es la aproximación que usaron Lucy \& Solomon (1970), Castor, Abbott, \& Klein (1975) y Abbott (1982b), en los albores de la teoría CAK. 
Si los fotones son todos radiales, en la ecuación 2.49, la forma de la intensidad específica será:

$$
I^{c}(\omega)=\frac{L_{\nu}}{4 \pi r^{2}} \delta(\breve{\boldsymbol{n}}-\breve{\boldsymbol{r}})
$$

donde la función delta de Dirac restringe todas las direcciones, excepto la radial $\breve{r}$. En tal caso, la integral sobre los ángulos sólidos de la ecuación 2.49 puede resolverse sencillamente dando, para la expresión de la aceleración radiativa en una única línea espectral:

$$
g_{\text {rad }}^{L}=\frac{\kappa_{L} v_{t h}}{c^{2}}\left(\frac{\nu_{0} L_{\nu}}{L_{*}}\right) \frac{L_{*}}{4 \pi r^{2}}\left[\frac{1-e^{-\tau_{0}}}{\tau_{0}}\right] .
$$

Si la línea en cuestión se encuentra cerca del extremo de la distribución de energía del continuo, el cociente $\nu_{0} L_{\nu} / L_{*}$ vale aproximadamente 1 .

El comportamiento de la aceleración radiativa es diferente para las líneas ópticamente delgadas que para las líneas ópticamente gruesas. Esta dependencia puede verse en la ecuación 2.51 como:

1. Líneas ópticamente delgadas $\left(\tau_{0} \ll 1\right)$ : El corchete de la ecuación 2.51 tiende a la unidad, entonces la aceleración para estas líneas (indicadas como opt. delgadas) queda:

$$
g_{\text {rad }}^{L}(\text { opt. delgadas })=\frac{\kappa_{L} v_{t h}}{c^{2}} \frac{L_{*}}{4 \pi r^{2}}
$$

En este caso, la dependencia con la inversa del cuadrado de la distancia permite unificar esta aceleración con la aceleración debida al continuo de radiación en una gravedad efectiva $g_{e f}$ :

$$
g_{e f}=g+g_{\text {rad }}^{C}+g_{\text {rad }}^{L}(\text { opt. delgadas })=-\frac{G M_{*}\left(1-\Gamma-\Gamma_{d e l}\right)}{r^{2}}
$$

donde:

$$
\Gamma_{d e l}=\frac{N_{d e l} \kappa_{L} v_{t h} L_{*}}{4 \pi c^{2} G M_{*}}
$$

y $N_{d e l}$ es el número de líneas ópticamente delgadas. De esta manera, la contribución de las líneas ópticamente delgadas consiste en disminuir la gravedad efectiva.

2. Líneas ópticamente gruesas $\left(\tau_{0} \gg 1\right)$ : El corchete de la ecuación 2.51 tiende a $\tau_{0}^{-1}$, por lo que, usando la definición de la ecuación 2.45, la aceleración para estas líneas (indicadas como opt. gruesas) queda:

$$
g_{\text {rad }}^{L}(\text { opt. gruesas })=\frac{\kappa_{L} v_{t h}}{c^{2}} \frac{L_{*}}{4 \pi r^{2}}\left(\frac{1}{\tau_{0}}\right)=\frac{L_{*}}{4 \pi r^{2} \rho c^{2}}\left(\frac{d v}{d r}\right) .
$$


En este caso, como las líneas son ópticamente gruesas, todas entran en resonancia con los fotones que han sufrido un corrimiento al rojo debido al gradiente del campo de velocidades, que entra linealmente en la aceleración. Se puede notar que aquí, la misma aceleración que establece el campo de velocidades depende, a su vez, del gradiente de la misma estructura de velocidades. Esta es una retroalimentación no lineal muy importante en la teoría de vientos impulsados por radiación.

Para calcular de una manera más adecuada la aceleración radiativa se deben tener en cuenta, simultáneamente, tanto líneas ópticamente gruesas como ópticamente delgadas.

\subsubsection{Aceleración radiativa para un conjunto de líneas espec- trales}

La aceleración radiativa debido a un conjunto de líneas en la aproximación de Sobolev tiene la forma dada por la ecuación 2.49. En esta ecuación se debe sumar sobre todas las líneas espectrales que contribuyen a la aceleración para todos los iones posibles. El cálculo directo de la aceleración con objeto de obtener la solución hidrodinámica del viento usando esa forma es completamente desventajosa, ya que implica la incorporación previa de una enorme cantidad de información sobre los niveles atómicos y datos relativos, junto a extraordinarios esfuerzos de cálculo. Distintos autores han propuesto algunas formas de enfrentar este problema, con diferentes grados de sofisticación.

Los primeros intentos exitosos fueron los de Lucy \& Solomon (1970), que sólo consideraron líneas intensas de resonancia. Luego Castor et al. (1975) extendieron la base de datos a numerosas líneas débiles. En ese trabajo, se propone que la aceleración del viento debido a la absorción en las líneas en una atmósfera en expansión radial puede escribirse como:

$$
g_{\text {rad }}^{L}=\frac{\sigma_{e} F}{c} \mathscr{M}(t),
$$

una expresión que puede obtenerse sumando los términos de la ecuación 2.51 para todas las líneas espectrales consideradas, y usando la definición de ancho Doppler $\Delta \nu_{D}$ (ecuación 2.31). En la ecuación 2.56, $F$ es el flujo integrado sobre las frecuencias y $\sigma_{e}$, la opacidad por dispersión de electrones. Nuevamente, la aceleración queda expresada en términos de la aceleración producida por el continuo, es decir, $\sigma_{e} F / c$. La contribución de las líneas está condensada en el factor $\mathscr{M}(t)$, llamado multiplicador de fuerza, que está definido como:

$$
\mathscr{M}(t)=\sum_{\text {lineas }} \frac{\Delta \nu_{D} F_{\nu}}{F} \frac{1}{t}\left(1-e^{-\eta t}\right),
$$

donde $F_{\nu}$ es el flujo emergente del disco estelar, $\eta$ es el cociente entre la opacidad de la línea y la opacidad de dispersión Thomson, dada por:

$$
\eta=\frac{\pi e^{2}}{m_{e} c} g_{u} f_{u l} \frac{n_{l} / g_{l}-n_{u} / g_{u}}{\rho \sigma_{e} \Delta \nu_{D}},
$$

en la cual, $m_{e}$ es la masa del electrón, $g_{u}, g_{l}, n_{u}$ y $n_{l}$ son los pesos estadísticos y las poblaciones de los niveles involucrados, respectivamente, donde el nivel de menor 
energía se indica con un subíndice $l$ y el de mayor energía, con subíndice $u$, y $f_{u l}$ es la intensidad de oscilador correspondiente a la línea espectral. Por otra parte, $t$ es el llamado parámetro de profundidad óptica para una atmósfera en expansión, y está definido (Castor 1974) como:

$$
t=\frac{\sigma_{e} \rho v_{t h}}{d v / d r}
$$

De este modo, la profundidad óptica de una línea en el viento está dada por la relación $\tau_{L}=t \eta$.

Las ecuaciones 2.56, 2.57 y 2.59 son estrictamente válidas en el límite en que la fotósfera estelar subtienda un ángulo sólido despreciable a la distancia a la cual es evaluada la aceleración de la línea. Para un radio próximo a la fotósfera, donde el tamaño del disco estelar es apreciable, los valores de la aceleración de la línea pueden reducirse hasta un $40 \%$ (Castor et al. 1975; Abbott 1982b).

En su trabajo original, Castor et al. (1975) se basaron en una lista de líneas espectrales y sus datos relativos para el ion de C III, a partir de la cual modelaron estadísticamente la forma del multiplicador de fuerza. Para esto usaron una ley de potencias de la profundidad óptica dada por la ecuación 2.59, de la forma:

$$
\mathscr{M}(t)=k t^{-\alpha}
$$

donde el ajuste a la solución completa se realiza a partir de los parámetros $k$ y $\alpha$. Estos parámetros pueden ser interpretados de diferentes maneras. En el caso de $k$, se lo interpreta como el número efectivo de líneas que contribuyen a la aceleración, o como la fracción neta de flujo fotosférico que sería bloqueado inmediatamente en la fotosfera, si todas las líneas fueran ópticamente gruesas (Puls et al. 2000). Respecto a $\alpha$, su interpretación directa es la proporción de líneas ópticamente gruesas a las ópticamente delgadas, respectivamente. Otra interpretación alternativa se presenta en la sección 2.2.7. como la pendiente de la distribución de intensidades de líneas espectrales.

Abbott (1982b) recalculó la aceleración radiativa en líneas, usando una extensa base de datos de transiciones atómicas en los primeros seis estados de ionización de todos los elementos químicos comprendidos entre el $\mathrm{H}$ y el $\mathrm{Zn}$, obteniendo los primeros parámetros de la fuerza de radiación calculados con precisión. Estos datos han sido extensamente empleados en la literatura.

\subsubsection{Incorporación del equilibrio de ionización}

Abbott (1982b) estudió la dependencia de la aceleración de radiación con la estructura de ionización del material a lo largo del viento. En general, los estados de ionización más bajos (es decir, los menos ionizados) tienen más líneas espectrales que contribuyen a la aceleración. El nivel de ionización decrecerá en la medida en que haya más cantidad de electrones libres disponibles para recombinarse. Por esta razón se encuentra que la aceleración radiativa es mayor cuando la densidad electrónica $n_{e}$ es también mayor. Esto puede describirse a partir de las ecuaciones que establecen el equilibrio de ionización que, en el caso de un viento estelar, toma una forma generalizada del Equilibrio Termodinámico Local, es decir, la ley de Saha (ecuación 5.46 de Mihalas 1978): 


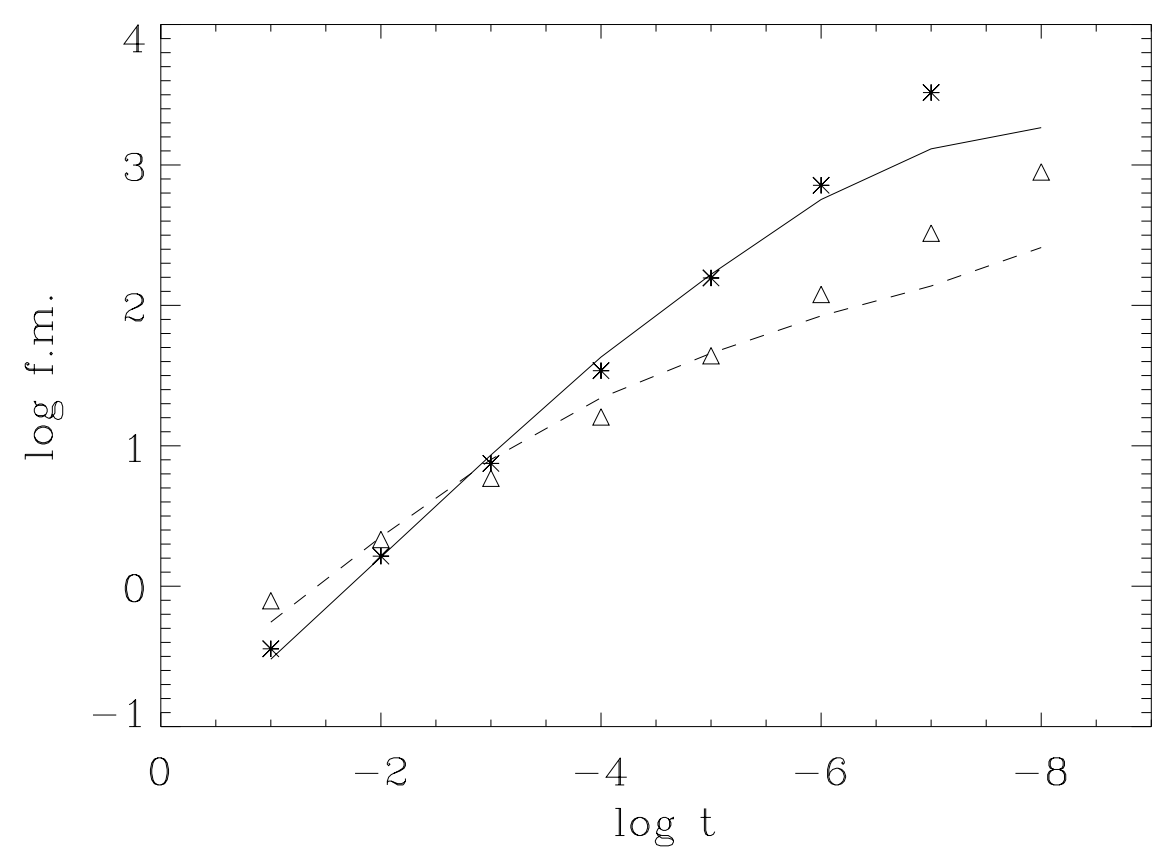

Figura 2.4. Logaritmo del multiplicador de fuerza como función de log $t$, para modelos con $\mathrm{T}_{\text {eff }}=50000 \mathrm{~K}$ (línea continua) y $10000 \mathrm{~K}$ (línea a rayas). En el gráfico puede observarse la forma de ley de potencias que toma $\mathscr{M}(t)$, siendo su pendiente proporcional al parámetro $\alpha$. Los asteríscos y los triángulos son ajustes lineales, calculados en el rango de $\log t$ comprendido entre -1 y -6. Figura tomada de Puls et al. (2000).

$$
\frac{n_{j}}{n_{j+1}} \approx \frac{1}{2}\left(\frac{h^{3}}{2 \pi m_{e} k_{B}}\right)^{3 / 2} \frac{n_{e}}{W(r)} \frac{1}{T_{r}\left(T_{e}\right)^{1 / 2}} \frac{U_{j}}{U_{j+1}} \exp \left(\frac{\chi_{j}}{k T_{r}}\right),
$$

que establece la proporción entre las poblaciones $n_{j}$ del estado de ionización $j$-ésimo, respecto a la población $n_{j+1}$ del estado $(j+1)$-ésimo. En la ecuación 2.61, $m_{e}$ es la masa del electrón, $k_{B}$ es la constante de Boltzmann, $T_{r}$ y $T_{e}$ son las temperaturas de radiación y electrónica, respectivamente, $U_{j}$ es la función de partición del estado de ionización $j$-ésimo y $\chi_{j}$, el potencial de ionización, o energía necesaria para pasar del estado $j$-ésimo al $(j+1)$-ésimo.

Resulta entonces que la proporción en la población de los iones depende directamente del cociente de la densidad electrónica $n_{e}$ y del factor de dilución, definido como:

$$
W(r)=\frac{\omega_{*}}{4 \pi}=\frac{1}{4 \pi} \int_{0}^{2 \pi} \int_{\mu_{*}}^{1} d \mu d \phi=\frac{1}{2}\left(1-\sqrt{1-\left(\frac{R_{*}}{r}\right)^{2}}\right),
$$

donde $\omega_{*}$ es el ángulo sólido subtendido por la estrella para el punto en el que se calcula la aceleración y $\phi$ es el ángulo acimutal. La variable $\mu$ se define como $\mu=\cos \theta$, donde el ángulo $\theta$ es medido desde el centro de la estrella hasta un punto cualquiera de su 


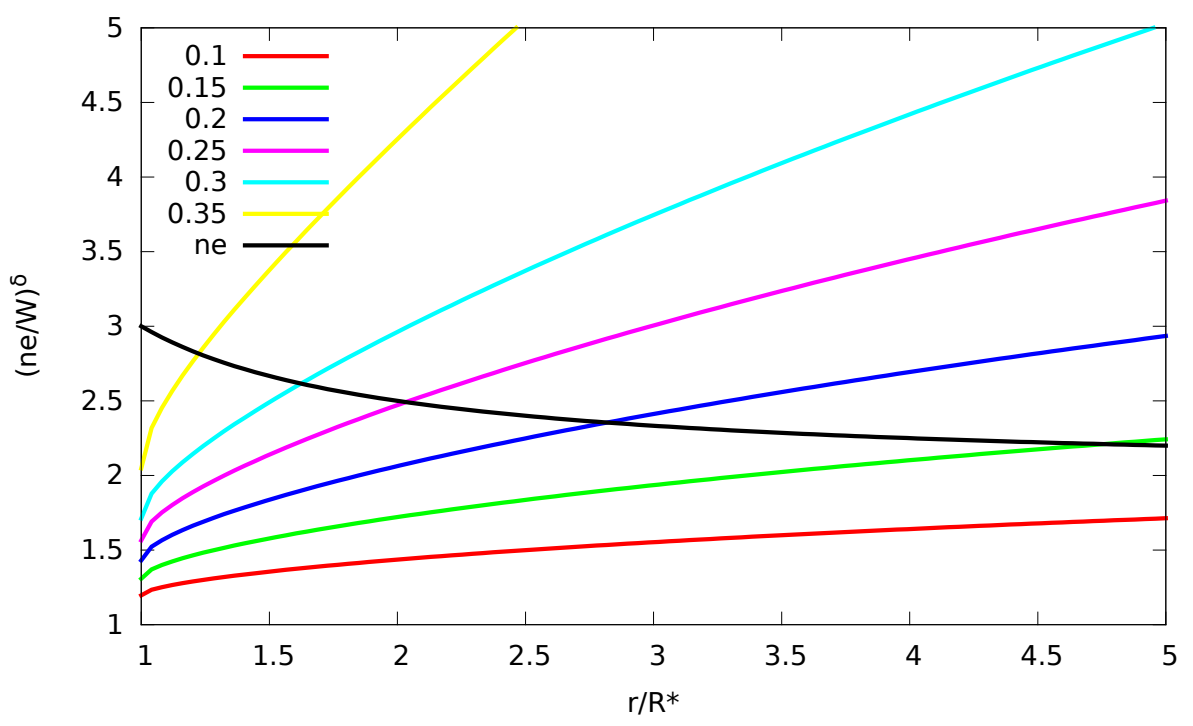

Figura 2.5. Visualización esquemática de la forma funcional del factor $\left(n_{e} / W(r)\right)^{\delta}$ introducido por Abbott (1982b), para una ley hipotética suavemente decreciente de la densidad electrónica con la distancia (curva negra). Los valores de $\delta$ para cada curva pueden verse en las referencias.

superficie, visto desde el punto donde se calcula la aceleración. Su valor máximo $\mu_{*}$ ocurre en el borde estelar y vale:

$$
\mu_{*}(r)=\sqrt{1-\left(\frac{R_{*}}{r}\right)^{2}} .
$$

Abbott (1982b) observó que el balance de ionización debería mantenerse aproximadamente constante a lo largo del viento, por lo que su influencia en la aceleración debería ser débil. Por este motivo, incorporó el parámetro $\delta$ al multiplicador de fuerza, como una potencia débil de la densidad electrónica $n_{e}$ y del factor de dilución, de la forma:

$$
\mathscr{M}(t)=k t^{-\alpha}\left(\frac{n_{e}}{W(r)}\right)^{\delta},
$$

donde la densidad electrónica está medida en unidades de $10^{-11} \mathrm{~cm}^{-3}$. La figura 2.5 muestra una visualización esquemática de la forma de esta incorporación adicional al multiplicador de fuerzas. En las condiciones de las estrellas O típicas, $\delta$ es pequeña (del orden de 0.1 , o menor). Sin embargo, $\delta$ puede alcanzar valores mucho mayores y aún negativos si ocurren en el viento grandes cambios en el equilibrio de ionización. Eso puede ocurrir en un viento denso o en un viento impulsado por $\mathrm{H}$ y $\mathrm{He}$ en el caso de las estrellas de metalicidad extremadamente baja (Puls et al. 2008b).

Si bien, en la ecuación 2.61, aparece una dependencia con las temperaturas, a través de $T_{r}$ y $T_{e}$, en la teoría de vientos impulsados por radiación, los vientos estelares son considerados casi isotermos, de modo que la dependencia con la distancia de esas variables no resulta significativa. 


\subsubsection{La función de distribución de intensidades de líneas es- pectrales}

A medida que la teoría de vientos impulsados por radiación fue progresando, se buscaron nuevas maneras de resolver la suma sobre las líneas espectrales de la ecuación 2.49. Siguiendo la sugerencia original de Castor et al. (1975), varios autores incorporaron una función de distribución de opacidades y de frecuencias para las líneas espectrales, en lugar de sumar directamente sobre todas las transiciones de líneas.

Owocki et al. (1988) propuso una forma para la distribución de intensidades de línea, independiente de la distribución en frecuencia. Esta distribución es:

$$
d N\left(\kappa_{L}\right)=\frac{1}{\kappa_{0}}\left(\frac{\kappa_{L}}{\kappa_{0}}\right)^{\alpha-2} e^{-\kappa_{L} / \kappa_{\max }} d \kappa_{L},
$$

que representa al número de líneas en un intervalo de frecuencias entre $\nu$ y $\nu+d \nu$ e intensidad de línea $\kappa_{L}$ comprendida entre $\kappa_{L} \mathrm{y} \kappa_{L}+d \kappa_{L}$. Esta forma incorpora a una función exponencial cuyo parámetro $\kappa_{\max }$ actúa como un corte para limitar el efecto de las líneas más intensas. El parámetro $\kappa_{0}$ tiene vinculación directa con el parámetro $k$ de Castor et al. (1975).

De manera alternativa, Puls et al. (2000) propuso usar una distribución de la forma:

$$
d N\left(\nu, \kappa_{L}\right)=-N_{0} f_{\nu}(\nu) \kappa_{L}^{\alpha-2} d \nu d \kappa_{L},
$$

relación en la cual el signo negativo tiene en cuenta que el número de líneas crece para intensidades de línea cada vez más bajas y $N_{0}$ es una constante de normalización, proporcional al parámetro $k$. Nuevamente, la distribución de frecuencias se supone que es independiente de la distribución de intensidades de línea. Si $\alpha=0$, todas las líneas serían ópticamente delgadas y si $\alpha=1$, todas las líneas serían ópticamente gruesas. Por lo tanto, para la situación intermedia en la que hay una mezcla de líneas ópticamente delgadas y gruesas, el parámetro $\alpha$ está comprendido en el intervalo $0<\alpha<1$ (Puls et al. 2008b).

Con las distribuciones de intensidades de línea propuestas, puede reemplazarse la suma sobre las líneas de la ecuación 2.49 con integrales, de la siguiente forma:

$$
\sum_{\text {lineas }} g_{\text {rad }}^{L} \rightarrow \int_{0}^{\infty} g_{\text {rad }}^{L} \frac{d N}{d \kappa_{L}} d \kappa_{L} .
$$

Tanto en los desarrollos usados por Puls et al. (2008b) como en los empleados por Owocki et al. (1988), el parámetro $\alpha$ tiene el mismo significado, siendo este parámetro, la pendiente de la distribución de intensidades de líneas espectrales. La interpretación del parámetro $\alpha$ como la proporción de líneas ópticamente delgadas a ópticamente gruesas tiene, en definitiva, el mismo significado que en el caso de la función distribución.

Resolviendo la integral para una distribución de intensidad de línea en la expresión derivada de la ecuación 2.49 (ver, por ejemplo, Cranmer 1996), el resultado general es:

$$
g_{\text {rad }}^{L}=\frac{\sigma_{e}^{1-\alpha} k}{c} \oint I^{c}(\omega)\left[\frac{\breve{\boldsymbol{n}} \cdot \nabla\left[\breve{\boldsymbol{n}} \cdot \boldsymbol{v}\left(\boldsymbol{r}^{\prime}\right)\right]}{\rho v_{t h}}\right]^{\alpha} d \omega
$$


que, en el caso de una fuente puntual, es decir, flujo puramente radial, toma la forma de:

$$
g_{\text {rad }}^{L}=\frac{\sigma_{e}^{1-\alpha} L_{*}}{4 \pi c r^{2}} \frac{k}{\left(\rho v_{t h}\right)^{\alpha}}\left(\frac{\partial v}{\partial r}\right)^{\alpha}
$$

semejante a la forma obtenida por Castor et al. (1975).

\subsubsection{Corrección por disco estelar finito}

Castor et al. (1975) propusieron por primera vez la corrección por disco estelar finito, en la cual se considera la presencia de fotones que llegan con ángulos diferentes desde la fotosfera, al punto donde se calcula la aceleración. Sin embargo, en ese trabajo no resolvieron las ecuaciones hidrodinámicas completas, sino que trataron solamente la aproximación de fuente puntual. Posteriormente, Friend \& Abbott (1986) simultáneamente con Pauldrach et al. (1986), resolvieron la ecuación de movimiento con la corrección por disco estelar finita, dando origen a la llamada teoría m-CAK o CAK modificada.

Para considerar a los fotones que incidan en direcciones no radiales, se parte de la ecuación 2.68, considerando la simetría en torno a un eje que pasa por el punto donde se calcula la aceleración y el centro de la estrella, como se muestra en la figura 2.2. Usando el elemento de ángulo sólido $d \omega=\operatorname{sen} \theta d \theta d \phi$ subtendido por la estrella desde el punto en cuestión, siendo $\theta$ el ángulo indicado en la figura 2.2 y $\phi$ el ángulo acimutal alrededor del eje; junto a la forma del gradiente de velocidades para una dirección $\mu$ dado por la ecuación 2.43, se obtiene (siguiendo los desarrollos de Friend \& Abbott 1986, Pauldrach et al. 1986):

$$
g_{r a d}^{L}=\frac{\sigma_{e}^{1-\alpha} k}{c} \oint I^{c}(\theta, \phi)\left[\frac{\mu^{2} \frac{\partial v}{\partial r}+\left(1-\mu^{2}\right) \frac{v}{r}}{\rho v_{t h}}\right]^{\alpha} \operatorname{sen} \theta d \theta d \phi .
$$

Por lo tanto:

$$
g_{\text {rad }}^{L}=\frac{\sigma_{e}^{1-\alpha} k}{c} \int_{0}^{2 \pi} \int_{-1}^{1} I^{c}(\mu, \phi)\left[\frac{\mu^{2} \frac{\partial v}{\partial r}+\left(1-\mu^{2}\right) \frac{v}{r}}{\rho v_{t h}}\right]^{\alpha} \mu d \mu d \phi,
$$

Para resolver la integral de esta ecuación, es necesario darle primero una forma a la intensidad específica $I^{c}(\mu, \phi)$, lo cual significa suponer algún tipo de distribución de brillo sobre la superficie estelar, por ejemplo, una ley de oscurecimiento al limbo. Por simplicidad, en este trabajo se desprecia ese efecto y se considera a la estrella como uniformemente iluminada. Entonces:

$$
I^{c}(\theta, \phi)=\left\{\begin{array}{lr}
\frac{L_{*}}{4 \pi R_{*}^{2}} \frac{1}{\pi}, & \mu_{*}<\mu<1 \\
0, & -1 \leq \mu<\mu_{*}
\end{array}\right.
$$

Por lo tanto, $I^{c}$ ya no es función de $\mu$ o $\phi$, en cuyo caso puede extraerse como constante de la integral. La integral sobre $\phi$ es directa y vale $2 \pi$.

En general, se define el factor de corrección por disco estelar finito (CF) como el cociente entre la aceleración en el caso de disco finito (ecuación 2.71) y la aceleración para una fuente puntual (ecuación 2.69): 


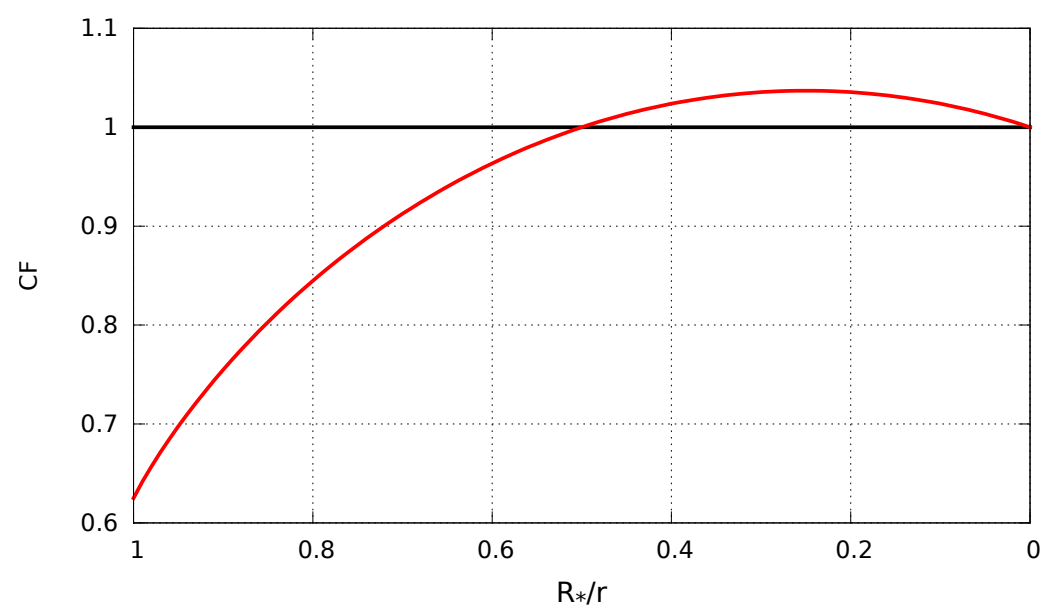

Figura 2.6. Factor de corrección por disco finito calculado a partir de la ecuación 2.77, a partir de un valor de $\alpha=0.6$ y una ley $\beta$ de velocidades (ecuación 2.2 ) con el parámetro $\beta=1$. Puede observarse que, para distancias grandes, este factor vale 1 , recuperándose la aproximación de fuente puntual. Por otro lado, en proximidades de la fotósfera, la aceleración radiativa resulta considerablemente disminuida.

$$
C F(r, v, \partial v / \partial r)=\frac{g_{\text {rad }}^{L}(\text { disco finito })}{g_{\text {rad }}^{L}(\text { fuente puntual })}=\frac{2}{(\partial v / \partial r)^{\alpha}} \frac{r^{2}}{R_{*}^{2}} \int_{\mu_{*}}^{1}\left[\mu^{2} \frac{\partial v}{\partial r}+\left(1-\mu^{2}\right) \frac{v}{r}\right]^{\alpha} \mu d \mu,
$$

donde $r^{2} / R_{*}^{2}=1 /\left(1-\mu_{*}^{2}\right)$ de acuerdo a la ecuación 2.63 .

La integral sobre $\mu$ restante puede resolverse con la ayuda del siguiente cambio de variables:

$$
\sigma=\frac{\partial \ln v}{\partial \ln r}-1=\frac{r}{v} \frac{\partial v}{\partial r}-1
$$

con lo cual, el integrando queda más simple de integrar:

$$
\mu^{2} \frac{\partial v}{\partial r}+\left(1-\mu^{2}\right) \frac{v}{r}=\left(\frac{1+\sigma \mu^{2}}{1+\sigma}\right) \frac{\partial v}{\partial r} .
$$

Por lo tanto, el factor de corrección queda definido como:

$$
C F=\frac{2}{1-\mu_{*}^{2}} \int_{\mu_{*}}^{1}\left[\frac{1+\sigma \mu^{2}}{1+\sigma}\right]^{\alpha} \mu d \mu,
$$

que, una vez integrado, se tiene:

$$
C F=\frac{(1+\sigma)^{1+\alpha}-\left(1+\sigma \mu_{*}^{2}\right)^{1+\alpha}}{\sigma(1+\alpha)(1+\sigma)^{\alpha}\left(1-\mu_{*}^{2}\right)} .
$$

Este factor de corrección tiende a 1 para $r \rightarrow \infty$, recuperándose la aproximación de fuente puntual, como puede verse en la figura 2.6. Cerca de la superficie fotosférica, 
la corrección es considerablemente menor que 1, y a cierta distancia, es mayor. Este comportamiento muestra que la corrección por disco finito afecta considerablemente la dinámica del viento, en relación con la aproximación de flujo puramente radial de CAK.

\subsubsection{Cálculo de modelos de vientos y parámetros de la ace- leración radiativa}

Varios autores han publicado parametrizaciones alternativas de la aceleración de radiación, con mayores o menores ventajas respecto a las originales de la teoría CAK, por ejemplo Gayley (1995) o Müller \& Vink (2008). Resulta importante notar que los nuevos parámetros han podido ser reescritos en función de los parámetros clásicos $k$, $\alpha$ y $\delta$. Por otra parte, los parámetros CAK tienen una interpretación simple y directa, como se vio en las sección 2.2.4, 2.2.6 y 2.2.7. Su extensa utilización en la literatura ha demostrado la fortaleza de estos parámetros en su formato original, justificando el uso de los mismos en el presente trabajo.

Son pocos los estudios en la literatura en los cuales se calcula directamente los parámetros de la fuerza de radiación; $k, \alpha$ y $\delta$ Abbott 1982b; Pauldrach et al. 1986, Krtička \& Kubát 2001). En general, en el modelado de los vientos los parámetros no son calculados consistentemente a lo largo del viento, sino que se imponen sus valores para un modelo y se mantienen fijos durante todo el cálculo. De acuerdo a Puls et al. (2008b), en la aplicación de la teoría de vientos impulsados por radiación, hay tres modos de abordar el análisis, estos modos son:

1. Obtención de espectros sintéticos a partir de modelos autoconsistentes que incluyen un tratamiento hidrodinámico de la estructura del viento, donde la aceleración es calculada consistentemente con los cálculos de los números de ocupación en NLTE y el correspondiente campo de radiación, a través de la resolución de la ecuación de transporte de radiación para medios en movimiento. Sus resultados son comparados con las observaciones con el fin de refinar los valores de entrada que, en general, son los parámetros fundamentales como la temperatura efectiva $\left(T_{\text {eff }}\right)$, la gravedad superficial $(\log g)$, el radio estelar $\left(R_{*}\right)$ y las abundancias químicas. Como desventajas, se trata de un abordaje complejo del problema, debido a que se requiere que cada parte del problema esté tratado con elevado nivel de detalle, los cálculos consumen mucho tiempo y se hace difícil localizar los errores en todo el proceso. Entre los pocos tratamientos de vientos realizados con esta metodología, pueden citarse los trabajos de Pauldrach et al. (1994) y Krtička \& Kubát (2001).

2. Generación de espectros sintéticos, obtenidos a partir de modelos hidrodinámicos y en la aproximación de NLTE, cuyo cómputo se inicia en la adopción de valores de prueba de los parámetros $k, \alpha$ y $\delta$, a partir de los cuales pueden obtenerse variables relevantes del viento, tales como la tasa de pérdida de masa o la velocidad terminal. Las características espectrales resultantes deben ser comparadas con las observadas, de modo de ajustar iterativamente los valores de los parámetros. El método tiene como ventaja principal, la mayor simplicidad respecto al enfoque anterior y la posibilidad de obtener una estratificación del campo de velocidades 
con mayor justificación física. Ejemplos de este tratamiento son los trabajos de Taresch et al. (1997) y Pauldrach et al. (2001).

3. El método más usado en el modelado de vientos estelares consiste en el desarrollo de su estructura a partir de un formato fijo de ley de velocidades, que es la ley $\beta$ (ecuación 2.2), y una transición suave entre la estructura analítica del viento y una fotosfera en quasi-equilibrio hidrostático. Los valores que se ingresan al cálculo son, directamente, los parámetros relativos al campo de velocidades tales como $\dot{M}, v_{\infty}$ y $\beta$. Nuevamente, los resultados de dichos modelos deben ser comparados con las observaciones, de modo de ajustar progresivamente, los parámetros del viento. Estos modelos son llamados Modelos de Atmósfera Unificados y fueron propuestos inicialmente por Gabler et al. (1989).

En esta tesis, se usa la segunda metodología, como se explicará en el capítulo 4. especialmente en la sección 4.3, y en el capítulo 6.

Los primeros valores de los parámetros de la aceleración radiativa fueron propuestos por Castor et al. (1975), siendo $k=1 / 30$ y $\alpha=0.7$. Algunos valores calculados por otros autores en el rango de valores de parámetros fundamentales de estrellas supergigantes de tipo B, pueden encontrarse en la Tabla 2.1. Generalmente, el parámetro $k$ adoptado en la literatura toma valores comprendidos entre 0.15 y 0.5 . En el caso de $\alpha$ los valores son más acotados, estando comprendidos entre 0.5 y 0.7 . Respecto a $\delta$, en general se adoptan valores menores o próximos a 0.1 , para simular una dependencia débil de la aceleración radiativa en la estructura de ionización del viento. Como referencias de la estimación de estos valores pueden citarse los trabajos de Abbott (1982b); Pauldrach et al. (1986); Puls et al. (2000).

\begin{tabular}{cccccc}
\hline \hline $\begin{array}{c}T_{\text {eff }} \\
{[K]}\end{array}$ & $\log g$ & $k$ & $\alpha$ & $\delta$ & Fuente \\
\hline 10000 & 1.5 & 0.36 & 0.54 & 0.05 & $\mathrm{~A} 82$ \\
15000 & 2.0 & 0.26 & 0.51 & 0.12 & $\mathrm{~A} 82$ \\
20000 & 2.5 & 0.48 & 0.51 & 0.089 & $\mathrm{~A} 82$ \\
& & 0.32 & 0.565 & 0.02 & $\mathrm{P} 86$ \\
30000 & 3.5 & 0.16 & 0.61 & 0.12 & $\mathrm{~A} 82$ \\
& & 0.17 & 0.59 & 0.09 & $\mathrm{P} 86$ \\
\hline
\end{tabular}

Tabla 2.1. Algunos parámetros de la aceleración de línea para estrellas supergigantes B, usados en la teoría estándar. La quinta columna de la tabla da las referencias de las que se extrajeron los valores de los parámetros. Los indicados con la denominación A82 son valores interpolados para $n_{e} / W=10^{11} \mathrm{~cm}^{-3}$ de la tabla 2 de Abbott (1982b), mientras que los citados como P86, corresponden a los publicados por Pauldrach et al. (1986).

Por otro lado, es importante notar que, en todos los trabajos mencionados, se considera que los parámetros $k, \alpha$ y $\delta$ se mantienen constantes a lo largo del viento. Esta condición puede no ser válida en los vientos impulsados por radiación. Sin embargo, como se argumenta en el trabajo de Puls et al. (2000), siempre es posible considerar 
un valor medio de dichos parámetros como representativo para la mayoría de los casos. Kudritzki et al. (1998) propuso una parametrización mejorada para la aceleración radiativa, con parámetros que varían de acuerdo a la posición a lo largo del viento. No obstante, el trabajo de Kudritzki et al. (1998) es puramente descriptivo y no ofrece resultados cuantitativos esperables para este tratamiento. Esta línea de investigación aún se encuentra sin explorar.

\subsubsection{Resolución de las ecuaciones hidrodinámicas en la teo- ría CAK}

Habiendo desarrollado una expresión adecuada para la aceleración radiativa en las líneas para fuentes puntuales (ecuación 2.69), junto con el factor de corrección por disco finito ( $\mathrm{CF}$, por ejemplo, ecuación 2.77), la ecuación hidrodinámica para el viento estelar (ecuación 2.17) queda:

$$
\left(v-\frac{a^{2}}{v}\right) \frac{d v}{d r}=\frac{2 a^{2}}{r}+\frac{G M_{*}(1-\Gamma)}{r^{2}}+g_{r a d}^{L},
$$

siendo

$$
g_{\text {rad }}^{L}=\frac{\sigma_{e}^{1-\alpha} L_{*} k}{4 \pi c r^{2}} C F\left(r, v, \frac{d v}{d r}\right)\left(\frac{1}{\rho v_{t h}} \frac{d v}{d r}\right)^{\alpha}\left[\frac{n_{e}}{W(r)}\right]^{\delta} .
$$

Usando la ecuación de continuidad en su forma integral (ecuación 2.7) y la definición de $\Gamma$ (ecuación 2.25), se obtiene de la ecuación 2.79.

$$
g_{r a d}^{L}=\frac{C}{r^{2}} C F\left(r, v, \frac{d v}{d r}\right)\left(r^{2} v \frac{d v}{d r}\right)^{\alpha}\left[\frac{n_{e}}{W(r)}\right]^{\delta},
$$

donde la constante $C$ es el autovalor de la ecuación a resolver, y está estrechamente vinculado a la tasa de pérdida de masa $\dot{M}$ y al parámetro $k$ :

$$
C=k G M_{*} \Gamma\left(\frac{4 \pi}{\sigma_{e} \dot{M} v_{t h}}\right)^{\alpha} .
$$

Debido a que el gradiente de velocidades aparece elevado a la potencia $\alpha$, la ecuación diferencial 2.78 resultante es fuertemente no lineal, y no puede resolverse analíticamente. Para su solución numérica se deben especificar las condiciones de contorno para esta ecuación. Primero se requiere que la velocidad, en algún punto cercano a la fotosfera estelar, sea baja (sónica) en comparación con la velocidad terminal; y segundo, que el flujo de material se conserve más allá del radio de Parker $\left(r_{P}=G M_{*} / 2 a^{2}\right)$. El análisis habitual consiste en examinar la ubicación de los puntos en el plano $\mathrm{r}-\mathrm{v}$ donde esta ecuación diferencial no lineal es singular.

Con el cambio de variables:

$$
\left\{\begin{array}{l}
u=v / a \\
u^{\prime}=d u / d r
\end{array}\right.
$$

la ecuación 2.78 puede escribirse como: 


$$
F\left(r, u, u^{\prime}\right)=0 \text {. }
$$

Esta ecuación tiene un punto crítico $\left(r_{c}, u_{c}\right)$ que debe buscarse en el plano $(r, u)$. En ese punto se deben cumplir la condición de singularidad, que establece que:

$$
\left[\frac{\partial F}{\partial u^{\prime}}\right]_{r_{c}}=0
$$

y debe cumplirse también allí, la condición de regularidad que implica que el gradiente de la velocidad en el punto crítico debe ser continuo. Esta condición se traduce en:

$$
\left[\frac{d F}{d r}\right]_{r_{c}}=\left[\frac{\partial F}{\partial r}+u^{\prime} \frac{\partial F}{\partial u}+u^{\prime \prime} \frac{\partial F}{\partial u^{\prime}}\right]_{r_{c}}=\left[\frac{\partial F}{\partial r}+u^{\prime} \frac{\partial F}{\partial u}\right]_{r_{c}}=0 .
$$

Las Ecs. 2.83, 2.84 y 2.85 pueden resolverse para obtener la velocidad crítica $u_{c}$, su derivada $u_{c}^{\prime}$ y la constante $\mathrm{C}$, que contiene la tasa de pérdida de masa. Esto se realiza por medio de un método iterativo que permite ubicar al punto crítico y su velocidad. A partir de ese punto se integran numéricamente las ecuaciones hacia adentro y hacia afuera, para obtener la solución completa del viento.

La solución de estas ecuaciones para las hipótesis de Castor et al. (1975) $(\mathrm{CF}=1)$, da una ley de velocidades de la forma:

$$
u=\left[\frac{2 \alpha G M_{*}(1-\Gamma)}{(1-\alpha) R_{*}}\left(1-\frac{R_{*}}{r}\right)\right]^{1 / 2},
$$

que es del tipo de la ley $\beta$ de velocidades, que se presentó en la ecuación 2.2, con $\beta=1 / 2$. Este resultado, obtenido por Castor et al. (1975), extendido a valores más altos de $\beta$ es usado extensamente para modelar los vientos estelares (ver sección 2.6.7).

Con este resultado, se fija la relación de escala:

$$
v_{\infty}=\left(\frac{\alpha}{1-\alpha}\right)^{1 / 2} v_{\mathrm{esc}}
$$

donde $v_{\text {esc }}$ es la velocidad de escape fotosférica, definida como:

$$
v_{\mathrm{esc}}=\left(\frac{2 G M_{*}(1-\Gamma)}{R_{*}}\right)^{1 / 2} .
$$

La relación de escala entre $v_{\infty} \mathrm{y} v_{\text {esc }}$ resultaba insuficiente para el trabajo original de Castor et al. (1975) (entre 1.1 y 1.3 veces, siendo que observacionalmente era del orden de 2 a 4 veces $v_{\text {esc }}$ ).

La incorporación de la corrección por disco finito, junto con los valores de los parámetros de la fuerza de radiación y la incorporación de la corrección por ionización sugerida por Abbott (1982b), permitieron que Pauldrach et al. (1986, m-CAK) mejorara considerablemente los valores de $v_{\infty}$. Suponiendo una expresión aproximada para la velocidad y la ubicación del punto crítico, junto a una ley $\beta$ de velocidades (ecuación 2.2), y usando una extensa grilla de modelos, (Pauldrach et al. 1986) determinaron un valor de ajuste al parámetro $\beta$ (en las capas por encima del punto crítico). Para estrellas con temperaturas efectivas comprendidas entre $T_{\text {eff }}=40000$ y $50000 \mathrm{~K}$, obtuvieron que $\beta=0.80$ quedando, para la ley de velocidades, la expresión: 


$$
v(r)=v_{\infty}\left(1-R_{*} / r\right)^{0.80}
$$

que es ampliamente usada en la literatura.

De este modo se ajustó apropiadamente la relación entre $v_{\infty} \mathrm{y} v_{\text {esc }}$, permitiendo que la teoría de vientos impulsados por radiación ganara un notorio impulso.

Para la tasa de pérdida de masa, Castor et al. (1975) obtuvieron una relación (sin factor de corrección por disco finito) un poco más complicada:

$$
\dot{M}=\left(\frac{4 \pi G M_{*}}{\sigma_{e} v_{t h}}\right) \alpha(1-\alpha)^{\frac{1-\alpha}{\alpha}}(k \Gamma)^{\frac{1}{\alpha}}(1-\Gamma)^{-\frac{1-\alpha}{\alpha}} .
$$

La predicción en $\dot{M}$ de Castor et al. (1975) resultó ser 2 a 3 veces mayor que los valores observados.

La teoría m-CAK mejoró considerablemente estos valores, en comparación con los obtenidos previamente. Incorporando el factor de corrección y el parámetro $\delta$ para la ionización, Pauldrach et al. (1986) obtuvieron la relación:

$$
\dot{M}=\left(\frac{c_{1}(2 \pi / D)^{\delta}\left(\sigma_{e} v_{t h} / 4 \pi\right)^{\alpha}}{k \Gamma G M_{*} 2^{-\delta / 2}}\right)^{1 /(\delta-\alpha)},
$$

siendo $c_{1}=\left(G M_{*}(1-\Gamma)\right)^{1-\alpha}\left(1.36 \times 10^{7} R_{*}^{2}\right)^{\delta}(1-\alpha)^{\alpha-1} \alpha^{-\alpha}(\alpha+1) 0.9\left(1-0.1^{\alpha+1}\right)$.

\subsection{La teoría estándar y los modelos unificados}

La teoría de vientos impulsados por radiación continuó su progreso en trabajos como el de Pauldrach (1987), donde se mejoró el cálculo de los números ocupacionales en NLTE para numerosos iones. Su resultado fue un aumento considerable de la ionización del material, dando una explicación parcial al fenómeno de la superionización. Este fenómeno, ya mencionado en la sección 1.1.1, implica la presencia de numerosos iones en altos estados de ionización ( $\mathrm{Si} \mathrm{IV}, \mathrm{N} \mathrm{V}$, O VI, etc.) en vientos de temperatura moderada. Las mejoras de Pauldrach (1987) permitieron reducir el requerimiento de fuentes importantes adicionales de fotones de alta energía tales como compañeras compactas o eventos de colisión de vientos.

Puls (1987) incluyó en el tratamiento de la aceleración radiativa, los efectos debidos a la superposición de líneas espectrales, es decir, que los fotones que provienen de la fotosfera puedan ser procesados más de una vez, en líneas diferentes. Esto puede producir un aumento en la transferencia de momento del campo de radiación al viento, en conjunción con un cambio en la intensidad media disponible y en los números ocupacionales. Puls (1987) probó la solución al problema con tres aproximaciones diferentes para calcular el campo de radiación: la aproximación de Sobolev, el transporte de radiación en el comoving frame y el método de Monte Carlo. Sus resultados demostraron que los efectos de multilínea son menores en proximidades a la fotosfera, debido a la reducción del flujo de fotones por la dispersión de los mismos hacia atrás. Lejos de la fotosfera, el efecto de la superposición de líneas se vuelve importante, reforzando la aceleración con los fotones reprocesados a lo largo del viento, proveniente de todos los ángulos, además de los subtendidos por el disco estelar. Como resultado completo, 
tanto las velocidades terminales como las tasas de pérdida de masa resultan reducidas respecto a los valores encontrados por la teoría m-CAK.

La teoría también fue refinada por Kudritzki et al. (1989), donde se presentó una receta (cooking recipe) para obtener las velocidades terminales y las tasas de pérdida de masa para vientos de estrellas tempranas, por medio de fórmulas directas, sin necesidad de contar con códigos especializados o de realizar todos los cálculos.

Los primeros modelos de los vientos de las estrellas tempranas consideraban dos regiones separadas con propiedades calculadas independientemente: la fotosfera y el viento, en una estructura llamada "núcleo-halo" (core-halo) sin otra conexión que las necesarias para la transición de un sistema a otro. Estas estructuras simples daban lugar a discrepancias en el ajuste de los perfiles de líneas tales como He II 4686 y $\mathrm{H} \alpha$, ya que las mismas surgen como una combinación de absorción y emisión procedente de ambas regiones: de la fotosfera y del viento. Entre los trabajos que encontraban estas dificultades se pueden citar: Kudritzki et al. (1983); Simon et al. (1983); Mendez et al. (1988).

La unificación de los modelos de fotosfera y viento, ambos en simetría esférica, fue llevada a cabo por Gabler et al. (1989), en lo que se conocen como modelos unificados. Estos modelos son estacionarios, con números ocupacionales en NLTE, en quasiequilibrio radiativo, y estructuras de densidad y de velocidad obtenidas mediante una transición suave entre la estratificación quasi-hidrostática de la fotosfera y la región del viento. Estos modelos tienen sus ventajas al conjugar información simultánea tanto del continuo fotosférico como de la radiación proveniente del viento mismo, modelando con mayor precisión tanto las líneas fotosféricas como las que se originan en la parte baja del viento. Esto es particularmente importante ya que algunas líneas tales como $\mathrm{H} \gamma, \mathrm{H} \delta$, He II 4542 y He II 4200 son usadas para las determinaciones de la gravedad estelar (sección 1.2.3), y pueden estar afectadas tanto por los efectos de esfericidad (que no pueden modelarse con fotosferas plano-paralelas), como por las contribuciones de emisión aportadas por el viento. Adicionalmente, los modelos unificados permitieron modelar mejor el salto del He II, localizado en $\lambda \lambda 228 \AA$, que resulta mucho mayor que en el caso de capas plano-paralelas. Este salto afecta sensiblemente la ionización del entorno en el caso de estrellas tempranas. Con estos avances, los modelos unificados se convirtieron en el estándar de la teoría de los vientos impulsados por radiación.

La próxima etapa para la aplicación de la teoría estándar fue el desarrollo de códigos de cálculo de atmósferas, como se resume en la sección 5.2, que actualmente brindan la posibilidad de confrontar los espectros sintéticos con los datos observacionales.

Uno de los últimos pasos en la optimización de los códigos fue la incorporación del "bloqueo de líneas" o line blanketing, que resume los efectos de una enorme multitud de líneas de metales en el UV y extremo UV (EUV) que actúan como una "manta" reduciendo el flujo radiativo que llega a las capas externas de la atmósfera. Simultáneamente, este efecto incrementa el campo medio de radiación y la temperatura electrónica de la región interna de la atmósfera, debido a la dispersión hacia atrás de los fotones (backscattering) y a la termalización (efecto conocido como backwarming). Como consecuencia, el grado de ionización aumenta, produciendo que las líneas de diagnóstico de temperatura tales como las de He se vuelvan más débiles ( $\overline{\mathrm{Puls}}$ 2008). Por lo tanto, en los modelos que no consideran el efecto de blanketing es necesario considerar temperaturas efectivas menores (al igual que gravedades menores, log g), para ajustar las 
observaciones en un determinado tipo espectral.

\subsection{Tratamientos alternativos de la aceleración de radiación}

Aunque la teoría estándar de vientos impulsados por radiación ha demostrado buenos resultados para modelar las observaciones (sección 2.5), aún subsisten numerosas discrepancias (sección 2.6). Eso ha motivado a algunos investigadores a buscar formas alternativas para describir el mecanismo de impulsión del viento y, más particularmente, la aceleración producida por las líneas espectrales.

Una de las modificaciones a la parametrización de la aceleración radiativa de la teoría CAK fue propuesta por Gayley (1995). Su objetivo fue indagar sobre el significado físico del parámetro $k$, que está relacionado con la influencia conjunta de la opacidad de las líneas espectrales que impulsan al viento, y que es determinante en la determinación de las tasas de pérdida de masa. Su aproximación consistió en introducir un nuevo parámetro adimensional $\bar{Q}$, casi constante y proporcional a la metalicidad. Este parámetro se define como:

$$
\bar{Q} \equiv \sum_{i} W_{i} q_{i}
$$

donde la suma se realiza sobre todas las líneas, y el coeficiente $W_{i}$ se define como:

$$
W_{i} \equiv \frac{\nu_{i} I_{\nu}(\mu)}{I(\mu)}
$$

siendo $\nu_{i}$ la frecuencia de cada línea y $I(\mu)=\int_{0}^{\infty} d \nu I_{\nu}(\mu)$, con $\mu=\cos \theta$. Además, el parámetro de intensidad de línea se define como:

$$
q_{i}=\frac{3}{8} \frac{\lambda_{i}}{r_{e}} f_{i} a_{i}
$$

donde $\lambda_{i}$ y $f_{i}$ son la longitud de onda y la intensidad de oscilador de cada línea, respectivamente, $a_{i}=n_{i} / n_{e}$ es el cociente de la población del nivel inferior involucrada en la transición y $n_{e}$, la población de electrones libres. La constante $r_{e}=e^{2} / m c^{2}$ es el radio clásico del electrón.

Con estas definiciones, el cociente entre la fuerza radiativa recibida por un electrón ligado (en un ion) y uno libre será:

$$
\frac{F_{\text {líneas }}}{F_{\text {libre }}}=\bar{Q} \bar{P}
$$

donde el factor $\bar{P}$ mide la reducción del flujo debido a la absorción de los iones adyacentes, dentro de una región de resonancia. Este factor se define como:

$$
\bar{P} \equiv \frac{\langle\bar{p}(\mu) \mu I(\mu)\rangle}{\langle\mu I(\mu)\rangle},
$$

en el que el factor $\bar{p}$ es la probabilidad de escape promediada: 


$$
\bar{p}(\mu) \equiv \frac{1}{\bar{Q}} \sum_{i} W_{i} q_{i} p_{i}(\mu),
$$

identificando a $p_{i}(\mu)$ con la probabilidad de escape definida en la ecuación 5.18 .

El cociente $F_{\text {líneas }} / F_{\text {libre }}$ es equivalente al multiplicador $\mathscr{M}(t)$ de fuerzas de la teoría CAK (ecuación 2.64). Pero en la aproximación de Gayley (1995), este factor se divide en dos componentes: $\bar{Q}$ que depende solamente de las características atómicas de las líneas involucradas y, por otro lado, el factor de corrección $\bar{P}$ de "autobloqueo" o (self-shadowing), que depende de la densidad del viento y de su dinámica. Esta parametrización representa un avance sobre la parametrización de la teoría CAK, ya que se diferencian las dos contribuciones principales mencionadas a la fuerza de radiación, que se encuentran aunadas en el parámetro $k$.

Para vincular la formulación de la fuerza de radiación de la teoría CAK con esta parametrización, puede escribirse que:

$$
\bar{Q} \bar{P} \equiv \mathscr{M}(t)=F k t^{-\alpha},
$$

donde el coeficiente $F$ se incluye para considerar el caso de estrella no puntual. Nótese que en esta expresión no se hace referencia al parámetro $\delta$ introducido por Abbott (1982b) para tener en cuenta los cambios en la ionización del viento.

Considerando una distribución de intensidades para las líneas (sección 2.2.7) con un corte $Q_{0}$ para la línea más intensa considerada, la equivalencia entre ambas parametrizaciones queda (para el caso de fuentes puntuales):

$$
k=\frac{1}{(1-\alpha)}\left(\frac{v_{\mathrm{th}}}{c}\right)^{\alpha} \bar{Q} Q_{0}^{-\alpha} .
$$

Este resultado puede convertir los parámetros de manera más sencilla, para el caso en que $Q_{0}=\bar{Q}$ (en el límite de la línea más intensa), por lo cual la ecuación 2.99 se transforma en:

$$
k=\frac{1}{(1-\alpha)}\left(\frac{T_{\text {eff }}}{5.5 \times 10^{12}}\right)^{\alpha / 2} \bar{Q}^{1-\alpha} .
$$

En la tabla 2.2 se comparan algunos valores de los parámetros CAK estándar con valores del parámetro $\bar{Q}$ de Gayley (1995).

\begin{tabular}{cccc}
\hline \hline $\mathrm{T}_{\text {eff }} / 10^{3}$ & $k$ & $\alpha$ & $\bar{Q} / 10^{3}$ \\
\hline $40 \mathrm{~K}$ & 0.174 & 0.606 & 1.7 \\
$30 \mathrm{~K}$ & 0.156 & 0.609 & 2.2 \\
$20 \mathrm{~K}$ & 0.531 & 0.506 & 1.4 \\
\hline
\end{tabular}

Tabla 2.2. Comparación entre los valores de los parámetros de Abbott (1982b) y los correspondientes valores de $\bar{Q}$ de Gayley (1995), para un viento con densidad intermedia.

En otro enfoque del problema, Müller \& Vink (2008) elaboraron una expresión alternativa para la aceleración en líneas espectrales como función de la distancia $r$ al 


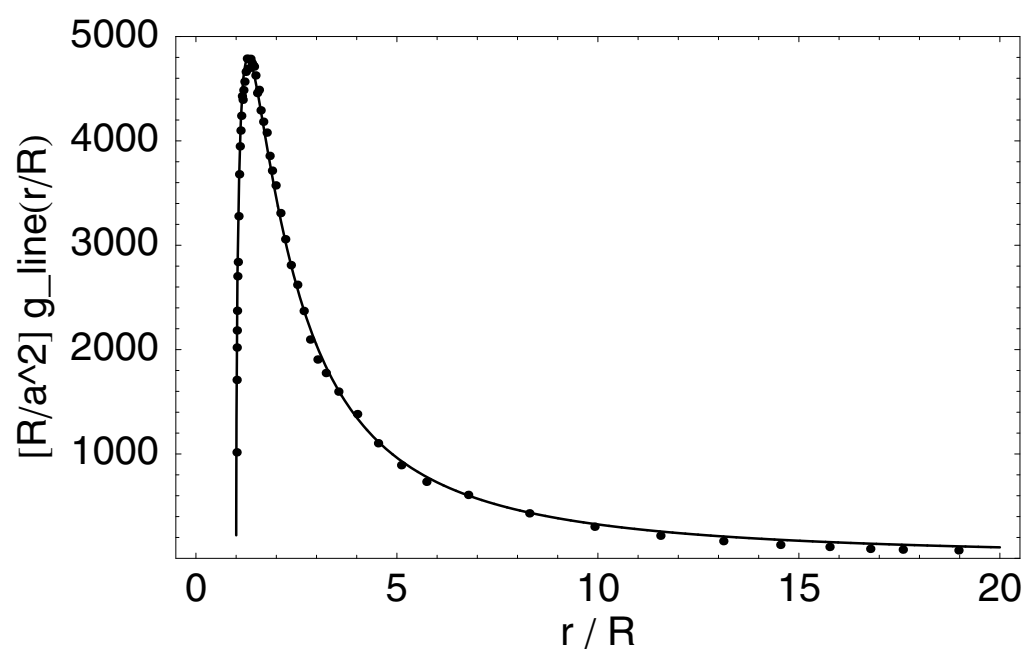

Figura 2.7. Aceleración radiativa sin dimensiones $\hat{g}_{\text {rad }}^{\text {linea }}$ en función de $\hat{r}$ para el viento de una estrella $\mathrm{O} 5 \mathrm{~V}$. Los puntos representan los resultados numéricos, mientras que la línea continua corresponde a un ajuste por medio de los parámetros $\hat{g}_{0}, \gamma, \delta$ y $\hat{r}_{0}$. Tomado de Müller \& Vink (2008).

centro del sistema solamente. Para esto, ensamblaron en una única función $\hat{g}_{\text {rad }}^{\text {líea }}(\hat{r})$, las propiedades físicas requeridas para este término de la ecuación de movimiento. En sus desarrollos, los autores reescriben la componente radial de la ecuación de movimiento con variables sin dimensiones, para un flujo estacionario en simetría esférica:

$$
\hat{v} \frac{d}{d \hat{r}} \hat{v}=-\frac{\hat{v}_{\text {crit }}^{2}}{\hat{r}^{2}}+\hat{g}_{\text {rad }}^{\text {línea }}(\hat{r})-\frac{1}{\rho} \frac{d \rho}{d \hat{r}},
$$

donde se define a $\hat{v}=v / a$, siendo $a$ la velocidad isotérmica del sonido, y $\hat{r}=r / R_{*}$. La cantidad $\hat{v}_{\text {crit }}=\left(G M_{*}(1-\Gamma) / R_{*}\right)^{1 / 2} / a$ es semejante a la velocidad de ruptura para una estrella rotante (ecuación 3.11). Con estas variables, la aceleración radiativa debida a las líneas espectrales es:

$$
\hat{g}_{\mathrm{rad}}^{\text {línea }}(\hat{r})=\frac{R}{a^{2}} g_{\mathrm{rad}}^{\text {línea }}(r) .
$$

Para construir la forma de $\hat{g}_{\text {rad }}^{\text {ínea }}(\hat{r})$, Müller \& Vink $(2008)$ requieren que esta aceleración se anule cerca de la fotosfera $\left(\hat{r}^{\prime}=0\right)$, que sea positiva para $\hat{r}>\hat{r}^{\prime}$, que decrezca como $1 / \hat{r}^{2}$ al aumentar la distancia radial $\hat{r}$ a la estrella central, y que tenga un máximo absoluto entre la superficie estelar y el borde externo del viento. Con estas condiciones, la forma de la parametrización queda:

$$
\hat{g}_{\text {rad }}^{\text {linea }}(\hat{r})=\frac{\hat{g}_{0}}{\hat{r}^{1+\delta}}\left(1-\frac{\hat{r}_{0}}{\hat{r}^{\delta}}\right)^{\gamma}=\frac{\hat{g}_{0}}{\hat{r}^{1+\delta(1+\gamma)}}\left(\hat{r}^{\delta}-\hat{r}_{0}\right)^{\gamma},
$$

la cual es independiente de $\hat{v}$ y $d \hat{v} / d \hat{r}$. Los parámetros de la fuerza son ahora: $\hat{g}_{0}, \gamma, \delta$ y $\hat{r}_{0}$, los cuales deben ajustarse para representar la forma funcional de la aceleración con la distancia radial $r$, como se muestra en la figura 2.7. Estos parámetros son tales que $\hat{r}^{\prime}=\hat{r}_{0}^{1 / \delta}, 0<\gamma \lesssim 1$ y $0<\delta \lesssim 1$. 
A partir de esta nueva parametrización, Müller \& Vink (2008) derivaron soluciones analíticas para la estructura de velocidad y de densidad para estrellas enanas $\mathrm{O}$ tempranas, haciendo uso de la llamada "función de Lambert W" para resolver la ecuación de movimiento. A diferencia de la teoría CAK, en este caso, el punto crítico de la ecuación de movimiento es directamente el punto sónico. La aceleración $g(r)$ fue ajustada mediante la técnica Monte Carlo (Abbott \& Lucy 1985) aplicada en los cálculos numéricos de transporte radiativo. Las tasas de pérdida de masa derivadas resultaron consistentes con las obtenidas previamente por Vink et al. (2000), mientras que el campo de velocidad resultó compatible con una ley de tipo $\beta$, semejante a los resultados de Pauldrach et al. (1986).

\subsection{Relaciones fundamentales de los vientos impul- sados por radiación}

A continuación se presentan dos relaciones fundamentales derivadas de la teoría de vientos impulsados por radiación: La relación Momento del viento y la Luminosidad (WLR) y relación Gravedad pesada en flujo y la Luminosidad (FGLR). Ambas permiten determinar la distancia a estrellas tempranas, a partir del análisis espectral mediante modelos de vientos desarrollados en el marco de la teoría.

\subsubsection{Relación Momento del Viento - Luminosidad (WLR)}

Como los vientos de las estrellas tempranas son impulsados por la transferencia de momento del campo de radiación, es esperable que haya una estrecha relación entre la luminosidad de la estrella y las características del viento resultante. Con ese concepto, se ha desarrollado uno de los mayores logros de la teoría de vientos impulsados por la radiación, que es la Relación Momento del Viento - Luminosidad. Esta relación fue extensamente estudiada en los trabajos de Puls et al. (1996), Kudritzki et al. (1997), Kudritzki et al. (1999) y Markova et al. (2004), entre muchos otros. La relación WLR puede obtenerse a partir de las relaciones de escala corregidas. Estas son: la velocidad terminal (ecuaciones 2.87 y 2.88):

$$
v_{\infty}=C_{\infty}\left(\frac{2 G M_{*}(1-\Gamma)}{R_{*}}\right)^{1 / 2}=C_{\infty} v_{\mathrm{esc}}
$$

siendo $C_{\infty} \approx 2.24 \alpha /(1-\alpha)$, y la relación para $\dot{M}$ (ecuación 2.90 , en la que se ha sustituido $\alpha$ por $\alpha^{\prime}=\alpha-\delta$ :

$$
\dot{M} \propto\left(k L_{*}\right)^{1 / \alpha^{\prime}}\left(M_{*}(1-\Gamma)\right)^{1-1 / \alpha^{\prime}},
$$

en la que se usa la proporcionalidad de $\Gamma$ con la luminosidad $L_{*}$ (ecuación 2.25). Con esas relaciones de escala puede construirse la cantidad definida como:

$$
D_{m o m}=\dot{M} v_{\infty}\left(\frac{R_{*}}{R_{\odot}}\right)^{1 / 2}
$$


llamada tasa modificada de momento del viento que, en virtud de las relaciones de escala (Ecs. 2.104 y 2.105) queda:

$$
D_{m o m} \propto\left(k \frac{L_{*}}{L_{\odot}}\right)^{1 / \alpha^{\prime}}\left(\frac{M_{*}}{M_{\odot}(1-\Gamma)}\right)^{\frac{3}{2}-\frac{1}{\alpha^{\prime}}} \propto\left(k \frac{L_{*}}{L_{\odot}}\right)^{1 / \alpha^{\prime}}
$$

la que fue simplificada considerando que $\alpha^{\prime}$ vale aproximadamente $2 / 3$. En esta ecuación puede verse que $D_{\text {mom }}$ es una cantidad independiente de la masa de la estrella y, a su vez, es una potencia de la luminosidad. Tomando logaritmos y llamando $\log D_{0}$ a la ordenada al origen y $x$ a la pendiente, queda una relación lineal:

$$
\log D_{m o m}=x \log \left(L_{*} / L_{\odot}\right)+\log D_{0},
$$

que es la Relación Momento del Viento - Luminosidad (WLR). Esta relación puede usarse para obtener distancias a estrellas galácticas o extragalácticas por medio de análisis puramente espectroscópicos (determinando $D_{m o m}$ ). También se la ha usado para determinar la dependencia empírica entre la tasa de pérdida de masa y la metalicidad en estrellas de tipo O en el Grupo Local (por ejemplo Mokiem et al. 2006, 2007b). Pero, fundamentalmente, su logro definitivo es confirmar la capacidad de la teoría de vientos impulsados por radiación de realizar predicciones confiables.

Resulta claro que, tanto el parámetro $\log D_{0}$ como el parámetro $x$, deben ser función del tipo espectral y de la clase de luminosidad de las estrellas en consideración. Por este motivo, se han llevado a cabo numerosas calibraciones de la WLR con objetos de diferentes grupos estelares tales como supergigantes O o CSPN. En la figura 2.8 se observa la calibración de la WLR para estrellas supergigantes azules, con los datos de ajuste de la Tabla 2.3 (Kudritzki \& Puls 2000). Las supergigantes O son las que tienen mayor momento del viento, el cual decrece para las B tempranas (B0 y B1) y aún más para las B intermedias (B1.5 a B3). Luego reaparece más intenso en las $\mathrm{A}$ supergigantes. La pendiente $x$ es más pronunciada para A y B intermedias que para las $\mathrm{O}$ supergigantes.

De acuerdo a la ecuación 2.107, la pendiente de la WLR es inversamente proporcional a $\alpha^{\prime}$, por lo tanto, su medida da un valor aproximado de este parámetro. Los valores obtenidos para los ajustes están presentados en la cuarta columna de la Tabla 2.3. $\alpha^{\prime}$ decrece al disminuir $T_{\text {eff }}$, con el consiguiente aumento de pendiente. En la interpretación física, se supone que el parámetro $\delta$ (de $\left.\alpha^{\prime}=\alpha-\delta\right)$ es prácticamente constante, por lo que el cambio está en la potencia $\alpha$ de la función de distribución de intensidades de línea. Achmad et al. (1997) propuso que las bajas velocidades terminales para las supergigantes B intermedias y tardías y las A supergigantes deberían requerir bajos valores de $\alpha$. Estas tendencias son atribuidas a los cambios en la ionización de los elementos, ya que los medios con baja ionizacón tienen iones con mayor número de líneas que absorben momento del campo de radiación, especialmente en la familia del Fe. Ese cambio es interpretado como el factor que modifica la pendiente de la distribución de intensidades de líneas (Kudritzki \& Puls 2000).

La variación de la ordenada al origen $\log D_{0}$ podría dar un indicio del número efectivo de líneas que impulsan al viento y, por lo tanto, una medida del parámetro $k$. Sin embargo, $\log D_{0}$ también dependen de $\alpha$ y de $\alpha^{\prime}$, lo cual hace más incierta la medida. 


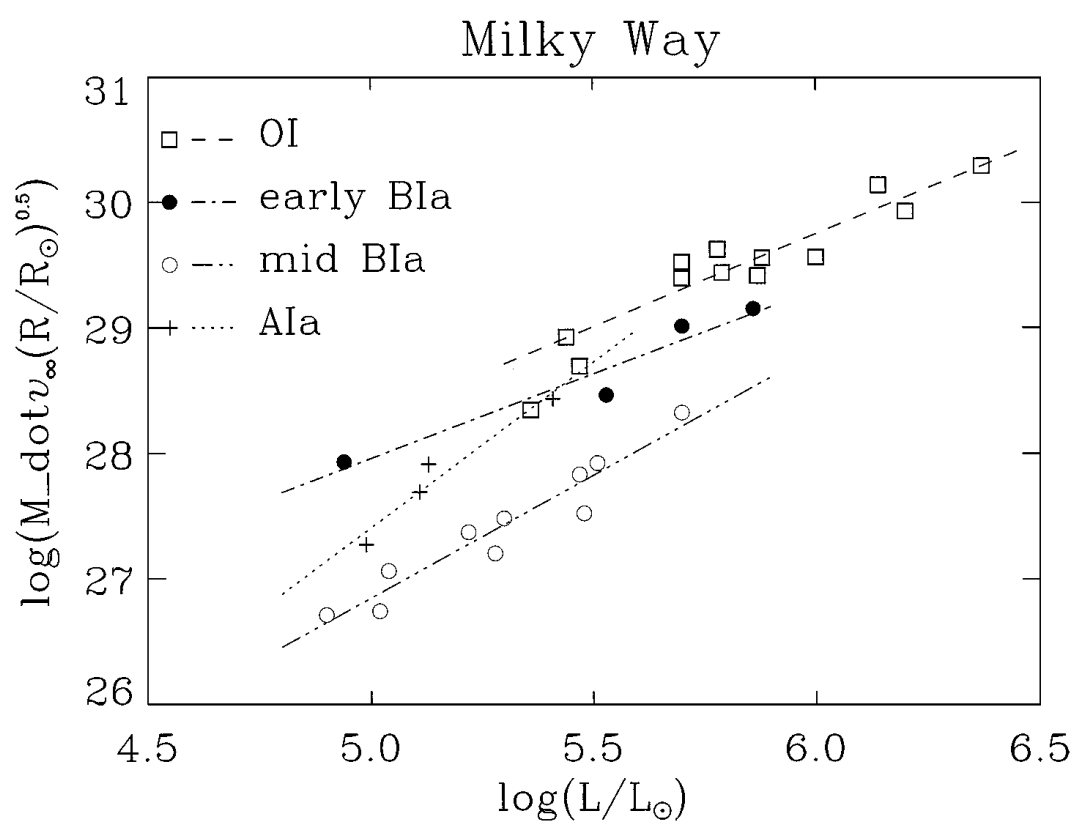

Figura 2.8. Relación empírica WLR para algunos grupos de estrellas supergigantes. Tomado de Kudritzki \& Puls (2000).

\begin{tabular}{lccc}
\hline \hline Clase Espectral & $\log D_{0}$ & $x$ & $\alpha^{\prime}$ \\
\hline AI & $14.22 \pm 2.41$ & $2.64 \pm 0.47$ & $0.38 \pm 0.07$ \\
BI intermedias & $17.07 \pm 1.05$ & $1.95 \pm 0.20$ & $0.51 \pm 0.05$ \\
BI tempranas & $21.24 \pm 1.38$ & $1.34 \pm 0.25$ & $0.75 \pm 0.15$ \\
OI & $20.69 \pm 1.04$ & $1.51 \pm 0.18$ & $0.66 \pm 0.06$ \\
\hline
\end{tabular}

Tabla 2.3. Valores de los parámetros del ajuste lineal a la WLR empírica. Tomado de Kudritzki \& Puls (2000).

\subsubsection{Relación Gravedad pesada en flujo - Luminosidad (FGLR)}

Durante su evolución hacia la etapa de las supergigantes rojas, las estrellas masivas atraviesan la fase de supergigantes B tardías y supergigantes A tempranas rápidamente y con una luminosidad y masa casi constante (Meynet et al. 1994, Meynet \& Maeder 2000, Heger \& Langer 2000). Esto significa que, en esta fase, la gravedad estelar $g \mathrm{y}$ la temperatura efectiva $T_{\text {eff }}$ están acopladas a la condición $g / T_{\text {eff }}^{4}=$ const. La cantidad $g / T_{\text {eff }}^{4}$ es llamada gravedad pesada en flujo (flux-weighted gravity). Suponiendo que la masa y la luminosidad siguen una relación usual $L \propto M^{q}$ (con q $\left.\sim 3\right)$, puede derivarse una relación entre la magnitud bolométrica $M_{\text {bol }}$ y la gravedad pesada en flujo, de la forma:

$$
-M_{\mathrm{bol}}=\operatorname{alog}\left(g / T_{\mathrm{eff}}^{4}\right)+b,
$$

donde el parámetro $a$ es del orden de -3.75. Esta relación, aplicada a los tipos espectrales 


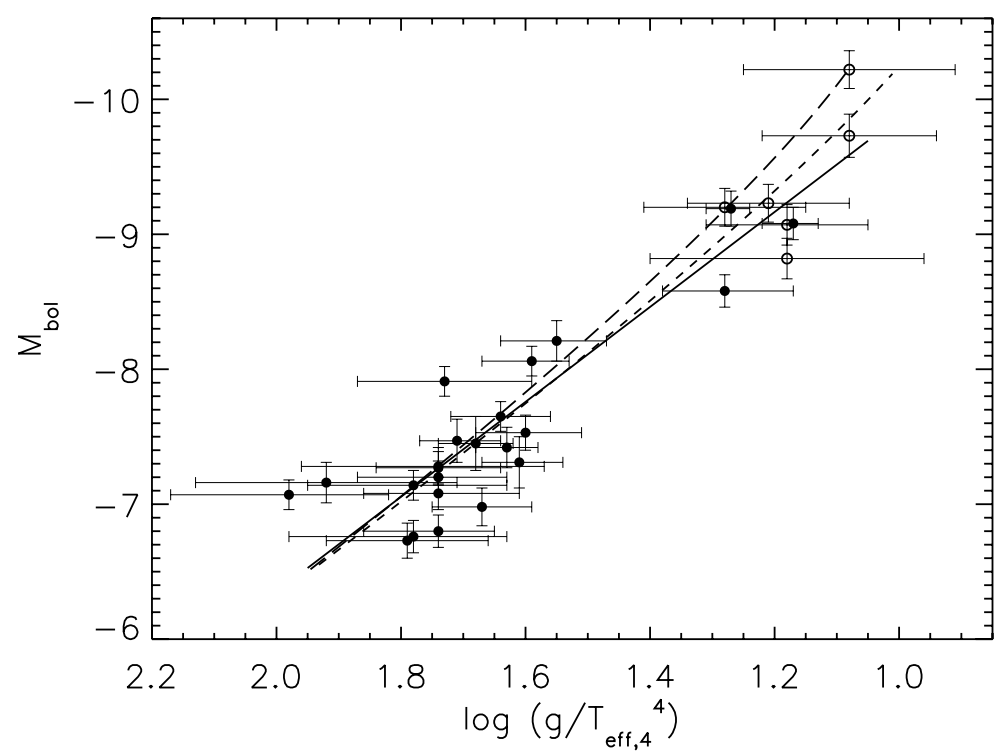

Figura 2.9. Relación Gravedad pesada en Flujo - Luminosidad (FGLR) (línea sólida) para estrellas supergigantes A (círculos llenos) y supergigantes B (círculos vacíos) en NGC 300. Las líneas de rayas corresponden a la relación derivada de modelos evolutivos con rotación, para las metalicidades de la Vía Láctea (rayas cortas) y de la Nube Menor de Magallanes (rayas largas). Tomado de Kudritzki et al. (2008).

adecuados, implica que los parámetros fundamentales gravedad y temperatura efectiva están fuertemente acoplados a la magnitud absoluta, posibilitando la determinación de distancias por medios puramente espectroscópicos.

La ecuación 2.109 se conoce como relación Gravedad pesada en Flujo - Luminosidad (FGLR, flux-weighted gravity-luminosity relationship) y fue propuesta por Kudritzki et al. (2003). En ese trabajo, los autores confirmaron la existencia de una relación de este tipo muy nítida en supergigantes azules del Grupo Local de galaxias.

Kudritzki et al. (2008) comprobaron nuevamente la validez de la FGLR con 24 supergigantes A de la galaxia NGC 300. En ese trabajo, combinaron los datos de su estudio previo sobre el Grupo Local de galaxias, obteniendo una relación bien definida. Posteriormente, Meynet et al. (2015) propusieron que, por ser una relación con poca dispersión, la FGLR puede servir para poner cotas a los modelos de evolución estelar. Con ese objetivo, investigaron si los modelos evolutivos pueden reproducir la FGLR, obteniendo un acuerdo razonable para una grilla de modelos estelares comprendida entre 9 y $40 \mathrm{M}_{\odot}$, con dos valores para las metalicidades y diferentes velocidades de rotación. Los mejores ajustes, tanto en la forma como en dispersión de los valores, fueron obtenidos para los modelos que incluyen los efectos de la rotación, y cuando se supone que la mayoría de los modelos involucrados se encuentran evolucionando hacia la fase de supergigantes rojas, sin volver a la región azul. Por su parte, también demostraron que los efectos de la metalicidad sobre la FGLR son pequeños. Este estudio le ha dado a la FGLR una fundamentación teórica sólida como un indicador de distancias extragalácticas. 


\subsection{Apartamientos de los vientos respecto a la teo- ría estándar}

La teoría estándar de vientos impulsados por radiación puede considerarse como una base conceptual sólida general sobre la cual se deben introducir nuevos fenómenos o correcciones, para poder dar cuenta de la gran variedad de vientos de las estrellas masivas. A lo largo del desarrollo de la teoría, la comparación de los parámetros observados de los vientos de las estrellas tempranas con las predicciones de la teoría, ha presentado numerosos problemas y discrepancias, los que han sido analizados y, en algunos casos, resueltos de diversas maneras. Muchos de estos apartamientos sólo han conseguido explicaciones parciales, mientras que otros, aún son objeto de mucha investigación y discusión. A continuación se presenta un resumen breve de las discrepancias más importantes.

Resulta interesante notar, en las subsecciones siguientes, que la mayoría de las discrepancias encontradas en los vientos impulsados por radiación, son atribuidas al menos parcialmente a fenómenos vinculados con la ionización del medio. Esto demuestra que el equilibrio de ionización es altamente complejo en estos objetos y fuente de mucha incerteza. Esto apoya la hipótesis de ionizaciones anómalas que genera soluciones hidrodinámicas alternativas, como las que se consideran en esta tesis para establecer la solución $\delta$-lenta (sección 3.3.4).

\subsubsection{Inestabilidades de los vientos impulsados por radiación}

Los vientos impulsados por radiación son intrínsecamente inestables. Debido a que la aceleración radiativa depende del gradiente de velocidades, cualquier pequeña perturbación en la velocidad o en la densidad, puede crecer espontáneamente, dando lugar a la formación de ondas de choque (shocks). Este comportamiento inestable se debe a que, si una fracción de gas se mueve con una velocidad levemente más alta que el material de sus alrededores, será capaz de absorber más radiación de la estrella debido a su corrimiento Doppler adicional. El efecto de esta absorción será acelerar ese material, impactando con el gas de su entorno, que se mueve más lentamente. De esta manera, se formará una onda de choque (Lamers 2001). Es de esperar que, en el interior de un viento, se formen estructuras de densidad y velocidad irregulares, entre las regiones impactadas y las intermedias rarificadas.

La inestabilidad de los vientos impulsados por radiación fue sugerida por primera vez por Lucy \& Solomon (1970), quienes propusieron que este mecanismo podría ser una de las explicaciones del fenómeno de superionización observado en los vientos de las estrellas tempranas (sección 2.3). MacGregor et al. (1979) y Carlberg (1980) derivaron las tasas de crecimiento lineal de las perturbaciones, suponiendo perturbaciones ópticamente delgadas en el gas. Abbott (1980) descubrió la formación de ondas llamadas ondas radiativo-acústicas u ondas de Abbott, que presentan dos modos de propagación, uno acústico lento, propagándose en dirección a la corriente de gas (downstream), y otro radiativo rápido, moviéndose en la dirección opuesta del flujo (upstream). El punto crítico encontrado por Castor et al. (1975) funciona como una barrera para estas ondas, tal como es el caso del punto sónico en las ondas de sonido (Holzer 1977). Esto 

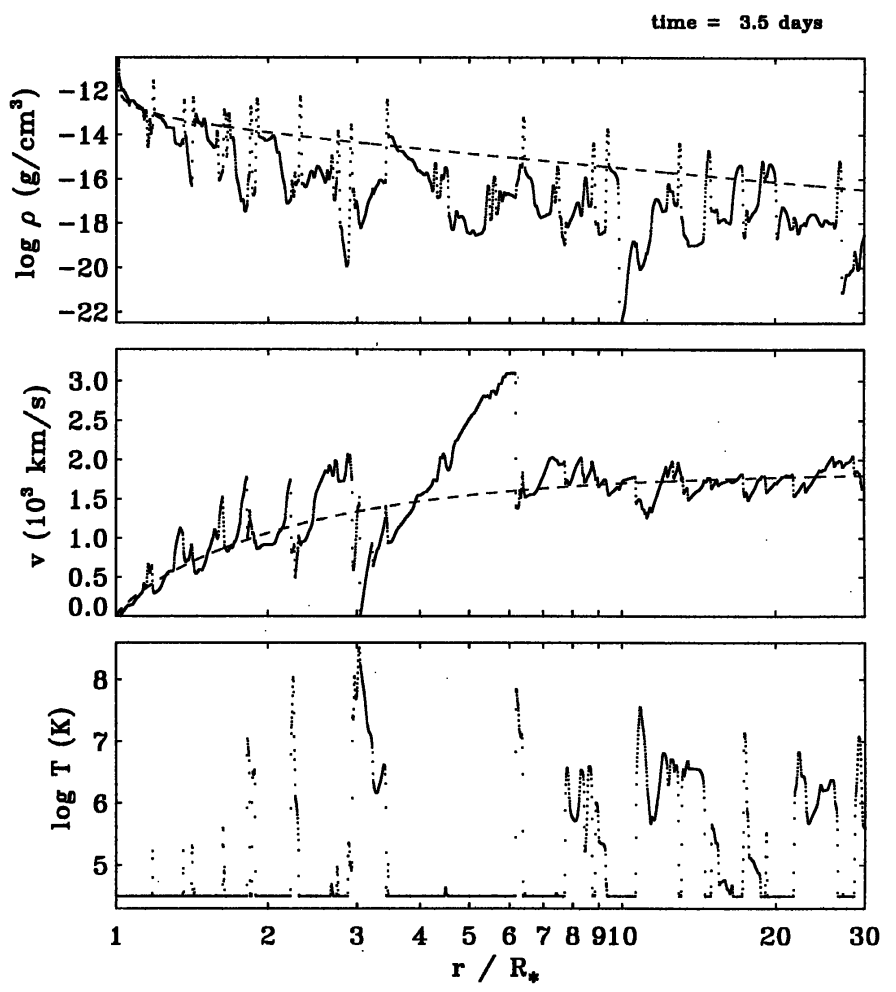

Figura 2.10. Inestabilidades calculadas para un viento impulsado por radiación, luego de 3.5 días de iniciada la perturbación en la región fotosférica. Se muestra el estado de las perturbaciones en la densidad (arriba), en la ley de velocidades (medio) y en la ley de temperaturas (abajo), y se superpone la estructura de un viento estacionario con $\beta$ $=0.8$ (línea a rayas). Tomado de Feldmeier et al. (1997)

implicaría que la tasa de pérdida de masa dependería puramente de los procesos que tengan lugar en la región subcrítica; mientras que la velocidad terminal del viento estaría determinada solamente por los procesos que ocurran en la parte super-crítica del viento (Puls et al. 2008b).

Por medio de un análisis detallado de la estabilidad lineal, Owocki \& Rybicki (1984) encontraron que la longitud de onda de las perturbaciones en comparación con la longitud de Sobolev $L_{\text {sob }}$ (ecuación 2.39) era fundamental para el crecimiento de la inestabilidad. Si la longitud de onda de la perturbación es menor o aproximadamente igual a $L_{\mathrm{sob}}$, el medio es inestable. Pero si la longitud de onda es mucho mayor que $L_{\mathrm{sob}}$, el medio resulta estable. Además, el análisis de primer orden confirmó la existencia de las ondas de Abbott.

El desarrollo de modelos permitió seguir la evolución temporal de las perturbaciones. La figura 2.10 muestra esta clase de perturbaciones para un tiempo de evolución de 3.5 días, calculadas por Feldmeier et al. (1997). Posteriormente, Feldmeier (1998) realizó el estudio de las tasas de crecimiento de las inestabilidades a partir de la aplicación de la aproximación de Sobolev hasta un segundo orden, cubriendo el régimen de perturbaciones que pueden desarrollar ondas de choque inversas, y que pueden calentar el gas hasta temperaturas suficientes como para generar rayos $\mathrm{X}$. 
Las ondas de Abbott fueron analizadas detalladamente por Feldmeier \& Shlosman (2002), quienes propusieron que las mismas podrían ser las responsables en dar forma a la ley de velocidad del viento y de fijar la tasa de pérdida de masa, eliminando las soluciones que no sean críticas, es decir, las que alcancen el punto crítico sin las condiciones de singularidad y regularidad requeridas (sección 2.2.10 y, por lo tanto, tendiendo a estabilizar la solución de las ecuaciones hidrodinámicas en una única solución estacionaria de tipo CAK.

De acuerdo a los modelos de las inestabilidades, las amplitudes de velocidad en las ondas de choque pueden ser del orden de $10^{2}$ a $10^{3} \mathrm{~km} \mathrm{~s}^{-1}$, y las fluctuaciones en densidad, pueden alcanzar los tres órdenes de magnitud, dando fuerte sustento teórico a la existencia del fenómeno del clumping (Lamers 2001). Este fenómeno es desarrollado en la sección 2.6.8.

\subsubsection{Mecanismo de bi-estabilidad}

El mecanismo de bi-estabilidad fue encontrado por primera vez por Pauldrach \& Puls (1990), a través del cálculo de modelos y su aplicación a la estrella P Cygni, una LBV extensamente estudiada cuyos perfiles de líneas espectrales le han dado el nombre a los perfiles compuestos (sección 1.1.1). Este mecanismo consiste en un cambio drástico de las propiedades del viento, $\dot{M}$ y $v_{\infty}$, para estrellas a un lado y a otro de una cierta temperatura efectiva (figura 2.11). Los primeros estudios lo situaban próximo a una temperatura efectiva del orden de los $21000 \mathrm{~K}$ (Lamers et al. 1995), aunque actualmente se lo ubica alrededor de los $22000 \mathrm{~K}$ (Markova \& Puls 2008).

Analizando una extensa muestra de estrellas, Lamers et al. (1995) reportó la existencia de una caida de un factor 2 en $v_{\infty}$, generando una discontinuidad en la escala de temperaturas efectivas. Vink et al. (1999) atribuyó este fenómeno a una variación brusca en el mecanismo de impulsión del material, que tiene lugar en el viento de las supergigantes, muy cercana a esa $T_{\text {eff. }}$ En su trabajo demostró que, a esa temperatura, el ion dominante del Fe IV recombina a Fe III, por lo que cambia de manera significativa la capacidad del viento para absorber momento del campo de radiación. Particularmente, el ion de estado más alto (Fe IV) tiene menos líneas que el ion de ionización más baja (Fe III), produciendo un incremento en las condiciones del material para absorber momento. Esto se traduce en un aumento de la densidad del material absorbente, incrementando la tasa de pérdida de masa y produciendo un descenso en la velocidad terminal del viento para las estrellas más frías del intervalo. De acuerdo a Pauldrach \& Puls (1990), el cambio ocurre cuando el continuo de Lyman del H se vuelve ópticamente grueso, impidiendo que la radiación UV penetre completamente en el viento e incrementando la recombinación del Fe IV en Fe III.

Los modelos de Vink et al. (1999) predecían que los valores de $\dot{M}$ se incrementaran en un factor 5, sin embargo, Markova \& Puls (2008) mostraron que la caida en la velocidad terminal no es compensada con un aumento suficientemente alto de la tasa de pérdida de masa. Este debate aún está abierto.

La abrupta ausencia de supergigantes B más tardías que $22000 \mathrm{~K}$, con alta rotación ( $v \sin i>50 \mathrm{~km} \mathrm{~s}^{-1}$ ), motivó a Vink et al. (2010) a sugerir que se trata de estrellas que podrían pertenecer todavía a la secuencia principal, pero que han sufrido la pérdida de su momento angular vía el incremento en la tasa de pérdida de masa producida en 


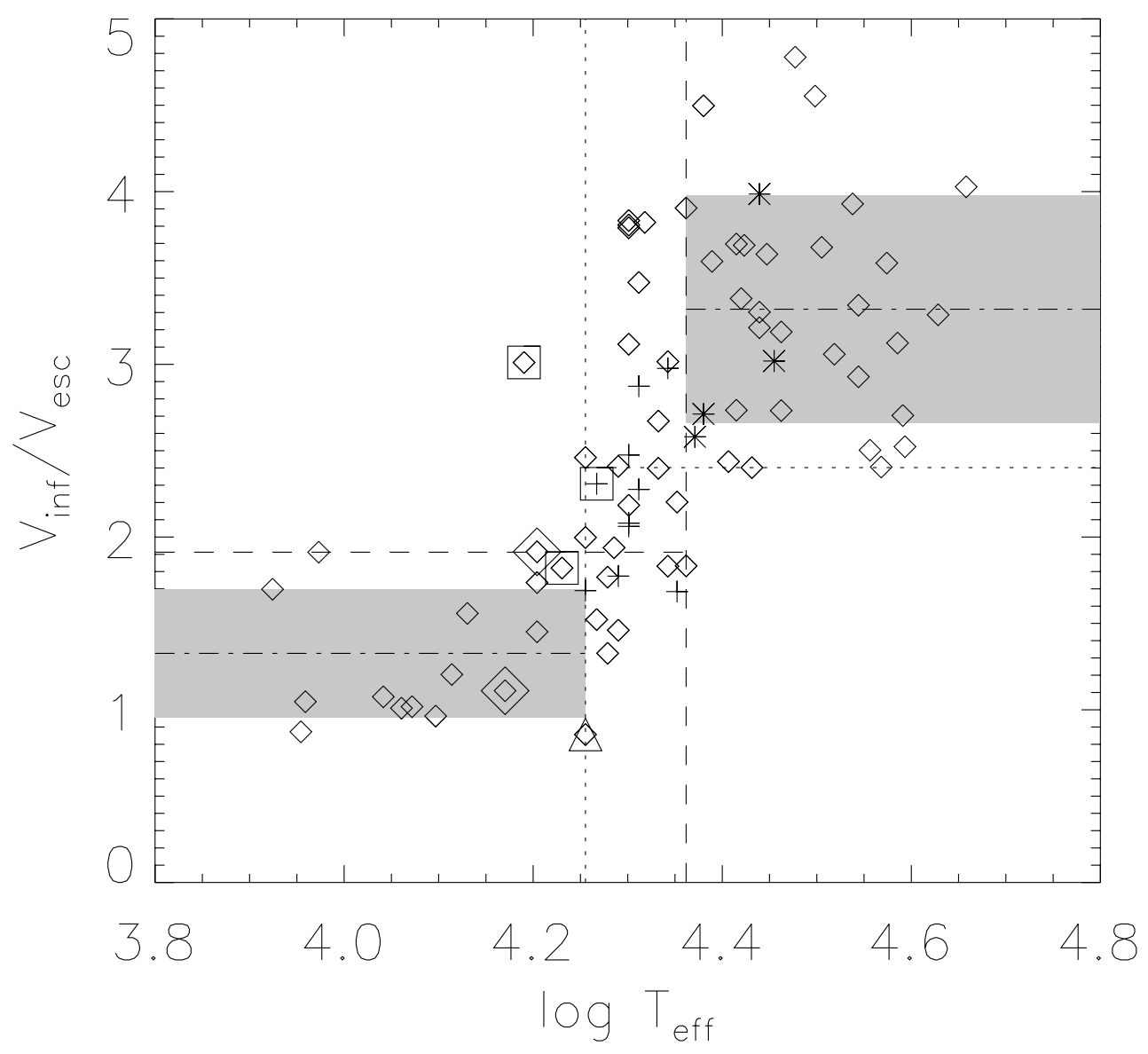

Figura 2.11. Salto de bi-estabilidad en $v_{\infty} / v_{\text {esc }}$ como función de $\log \mathrm{T}_{\text {eff }}$. Las supergigantes OBA se indican con diamantes, astericos o signos positivos; los objetos de clasificación dudosa, con triángulos, cuadrados y rombos. En el gráfico se puede notar que la región de transición no es completamente abrupta, sino que abarca un rango angosto de temperaturas efectivas. Tomado de Markova \& Puls (2008)

el momento evolutivo de cruzar el salto de bi-estabilidad. A este fenómeno se lo llama frenado de bi-estabilidad (bi-stability braking).

\subsubsection{Discrepancia de masas}

Herrero et al. (1992) encontraron una importante discrepancia entre las masas derivadas espectroscópicamente (obtenidas vía log g) para las estrellas O galácticas evolucionadas, respecto a los valores obtenidos a partir de los cálculos evolutivos. Las masas derivadas mediante estos modelos tienen valores sistemáticamente mayores por, aproximadamente, un factor dos. El análisis original fue hecho con modelos de atmósfera plano paralelos, sin blanketing (sección 2.3), incorporando sólo H y He. Una mejora parcial fue obtenida mediante la inclusión de rotación en los cálculos de las masas evolutivas (por ejemplo, Maeder \& Meynet 2000), aunque esta aproximación no alcanza por sí misma para resolver completamente la discrepancia. La inclusión de blanketing 
y geometría esférica en modelos con viento por parte de Repolust et al. (2004), ha disminuido considerablemente la diferencia, casi eliminándola en la mayoría de los casos de supergigantes O. Sin embargo, una diferencia en las masas aún subsiste para muchas enanas y gigantes $\mathrm{O}$ (Puls 2008), como puede verse en la figura 2.12, tomada de Mokiem et al. (2007a).

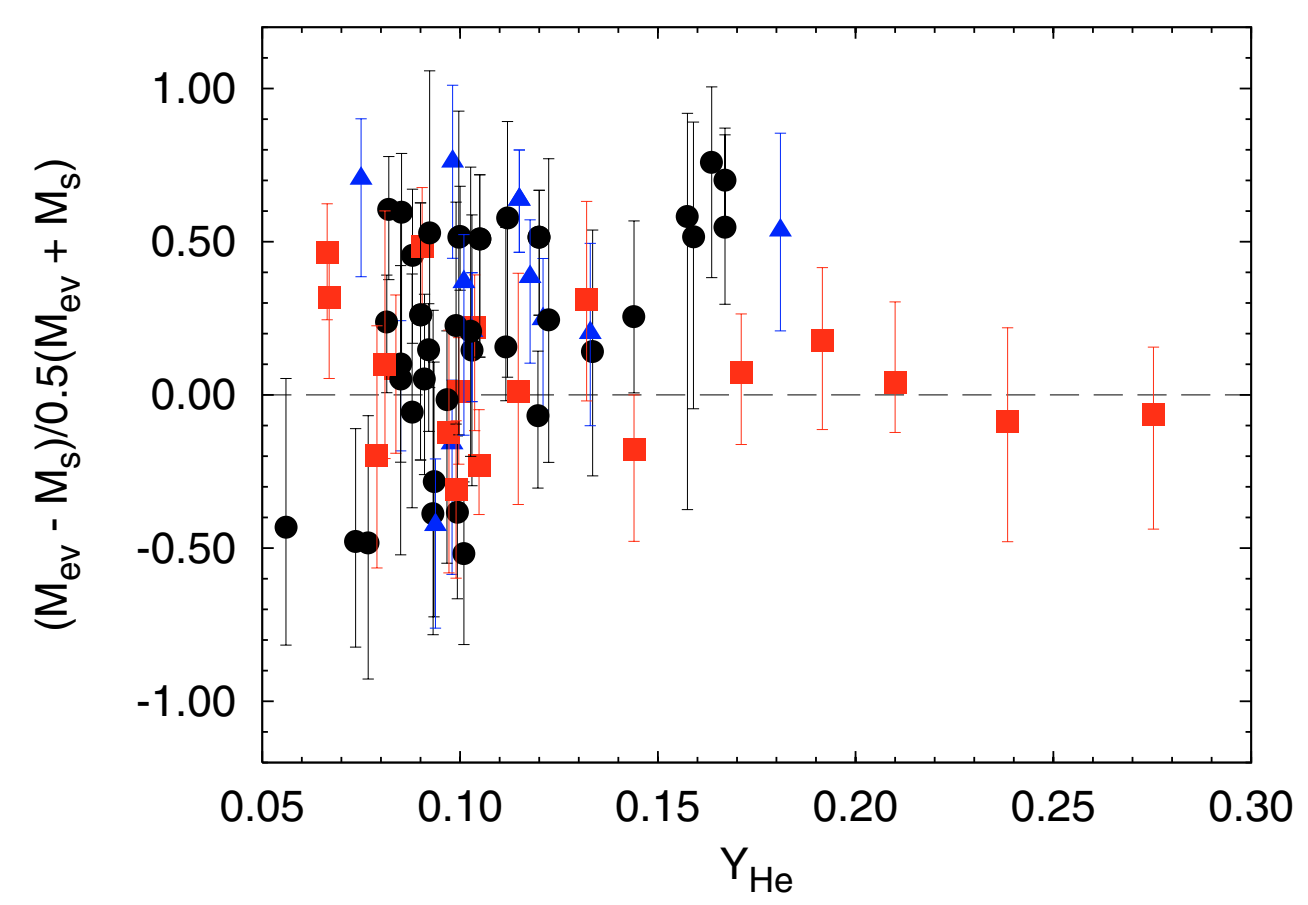

Figura 2.12. Discrepancia de masas en función de la abundancia de He para una muestra combinada de estrellas enanas (círculos negros), gigantes (triángulos azules), gigantes brillantes y supergigantes (cuadrados rojos) galácticas, de la Nube Menor y de la Nube Mayor de Magallanes. Puede verse la buena correspondencia entre la masa espectroscópica $\left(\mathrm{M}_{s}\right)$ y la masa evolutiva $\left(\mathrm{M}_{e v}\right)$ para gigantes brillantes y supergigantes (cuadrados), pero una gran dispersión para los objetos de la secuencia principal y algunas gigantes. Tomado de Mokiem et al. (2007a)

En la figura 2.12, la discrepancia en la masas está representada con relación al contenido de He, que es un indicador del estado evolutivo. La figura muestra buen acuerdo para las estrellas supergigantes y gigantes luminosas, aunque se mantiene una diferencia para las enanas y gigantes, la cual crece al aumentar la abundancia de He. Los autores de ese trabajo sugieren que hay una mezcla eficiente en la fase de la secuencia principal, dando lugar a una evolución químicamente homogénea.

\subsubsection{La macro-turbulencia y la rotación}

Para estimar las velocidades de rotación proyectadas, $v$ sin $i$, se requiere la medición cuidadosa de los anchos de líneas de metales formadas en la fotosfera. Sin embargo, los estudios han indicado la presencia de procesos adicionales a la rotación, si se intenta 
dar cuenta del ensanchamiento observado de algunas líneas espectrales de estrellas $\mathrm{O}$ (Conti \& Ebbets 1977). Por este motivo se introdujo, en los modelos, el fenómeno de la macro-turbulencia, caracterizado por adicionar una velocidad de macro-turbulencia $\left(\mathrm{v}_{\mathrm{ma}}\right)$, al cálculo del ensanchamiento de los perfiles. Esta velocidad debe distinguirse de la micro-turbulencia, abreviada como $\mathrm{v}_{\text {turb }} \mathrm{O} \mathrm{v}_{\mathrm{mi}}$, que surge de la agitación térmica de los iones. La forma característica de los perfiles ensanchados por macro-turbulencia es la de una letra "V", lo que claramente no se corresponde con un perfil de tipo rotacional (figura 2.13).

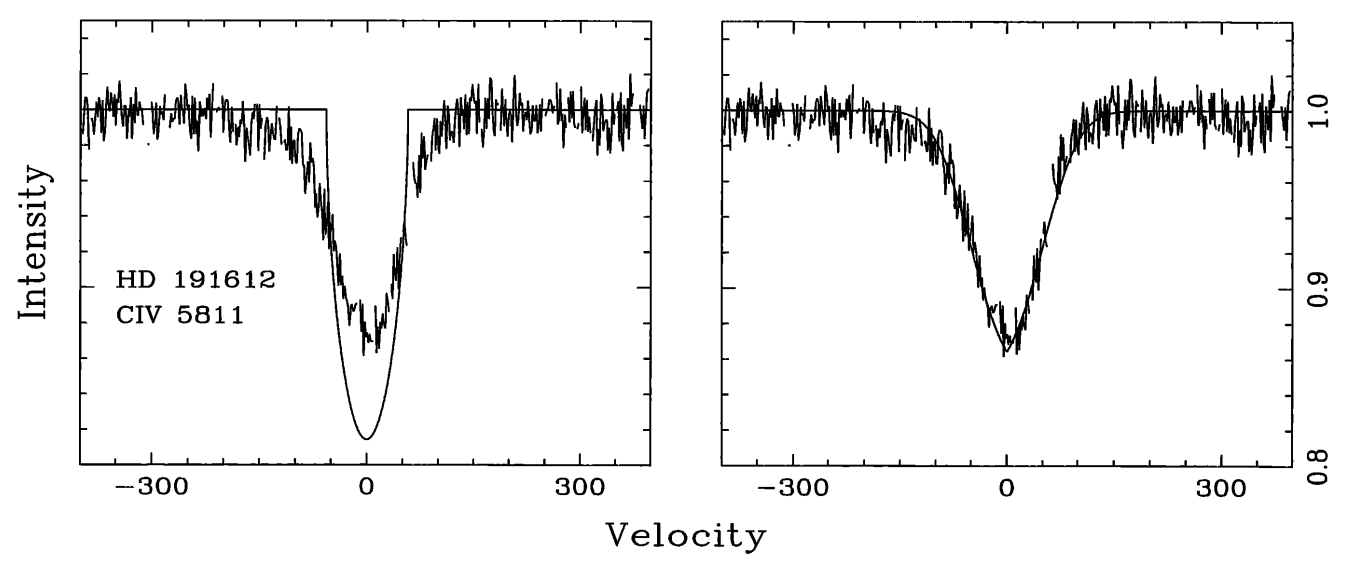

Figura 2.13. Perfil de la línea C IV $\lambda 5811$ del espectro de la estrella O HD 191612 , mostrando una característica forma de letra "V". A la izquierda se compara el perfil observado con el resultante de un modelo con rotación pura, para el mismo ancho a mitad de profundidad y ancho equivalente. A la derecha, el mismo perfil ha sido ajustado con un perfil sintético generado considerando macro-turbulencia. Tomado de Howarth (2004)

Un fenómeno similar fue encontrado en las supergigantes B tempranas e intermedias, aunque no en las enanas B (Lennon et al. 1993). Analizando una extensa muestra de estrellas tempranas observadas con el satélite IUE, Howarth et al. (1997) también encontraron evidencias de otro mecanismo que incrementa los ensanchamientos de las líneas espectrales por encima de la rotación y que, por analogía, se lo interpretó como macro-turbulencia. Posteriormente, Ryans et al. (2002) mostraron que este ensanchamiento adicional puede describirse con un perfil gaussiano con velocidades típicas del orden de $50 \mathrm{~km} \mathrm{~s}^{-1}$.

El valor de $v$ sin $i$ puede estimarse a partir del primer mínimo de la transformada de Fourier del espectro (Gray 2005), por lo que, el ajuste de la forma del perfil teórico a uno observado puede dar una indicación del valor de $\mathrm{v}_{\mathrm{ma}}$ (Simón-Díaz \& Herrero 2007, 2014). Las mediciones de la velocidad de macro-turbulencia en supergigantes OB sugieren que los valores de $v \sin i$ se ha sobreestimado entre 20 a $40 \mathrm{~km} \mathrm{~s}^{-1}$ (Lefever et al. 2007; Markova \& Puls 2008). La situación para las enanas es todavía indefinida.

La presencia de macro-turbulencia ha sido atribuida a fenómenos oscilatorios en la fotosfera estelar (Lucy 1976, Howarth 2004, Kraus et al. 2015a). Por medio de simulaciones de perfiles de línea, Aerts et al. (2009) propusieron formas para diagnosticar si está presente el ensanchamiento pulsacional en los perfiles de línea observados. 
A partir del estudio de una extensa base de datos de estrellas $\mathrm{O}$ y B obtenidas con el mismo instrumental y en las mismas condiciones (Proyecto IACOB), Simón-Díaz et al. (2017) concluyeron que la causa de los ensanchamientos observados es el efecto combinado de (a) modos pulsacionales asociados con un mecanismo de transporte de calor, y (b) movimientos cíclicos superficiales, iniciados por inestabilidades turbulentas en la presión.

\subsubsection{Problema de los vientos débiles}

Uno de los desafíos más importantes de la teoría de vientos impulsados por radiación es interpretar la presencia de vientos débiles (weak winds) ópticamente delgados, en estrellas O. Las primeras referencias fueron hechas por Chlebowski \& Garmany (1991), quienes encontraron que las tasas de pérdida de masa de las estrellas enanas $\mathrm{O}$ de tipos espectrales tardíos, eran significativamente menores (al menos en un factor 10) que las predichas a partir de las mediciones de los flujos en rayos X. Para ser definidos como vientos débiles, las tasas de pérdida de masa deben ser menores que $10^{-8} \mathrm{M}_{\odot}$ año ${ }^{-1}$ (Marcolino et al. 2009).

Si bien se trata de una discrepancia propia de las estrellas $\mathrm{O}$ enanas, algunas gigantes B también las han presentado, como surge del análisis de las líneas UV de $\beta$ CMa (B1 II) o de las observaciones en rayos $\mathrm{X}$ del satélite ROSAT para $\epsilon$ CMa (B2 II) (Drew et al. 1994).

Las estrellas $\mathrm{O}$ con vientos débiles presentan principalmente un espectro en absorción, con algunas pocas líneas de viento en emisión, tales como C IV $\lambda \lambda$ 1548, 1551. En cambio, en enanas $\mathrm{O}$ intermedias y tempranas, estas líneas presentan perfiles P-Cygni, a las que se agregan N IV $\lambda \lambda 1718, \mathrm{~N}$ V $\lambda \lambda 1239,1243$ o O V $\lambda \lambda 1371 \AA$.

Las diferencias entre los valores observados de $\dot{M}$ y los predichos por la teoría han sido atribuidos a diferentes causas posibles (Kudritzki \& Puls 2000):

- La disminución de la fuerza de radiación producida por la ionización debida a rayos X generados en shocks (Drew et al. 1994).

- El bloqueo por parte de líneas fotosféricas de radiación que no alcanza a llegar al viento y dar su contribución a la aceleración (line shadowing), este efecto puede ser común, especialmente, en las enanas B (Babel 1996).

- El desacople coulombiano de los iones respecto al plasma que ocurre a alguna densidad crítica (ver, por ejemplo, Krtička et al. 2006).

- En las estrellas con baja tasa de pérdida de masa y continuo ópticamente delgado en la región transónica, los términos de curvatura de las leyes de velocidad podrían producir gradientes en la función fuente, reduciendo la fuerza de radiación $(\mathrm{Puls}$ et al. 1998; Owocki \& Puls 1999).

Martins et al. (2004) analizaron estas propuestas para una muestra de estrellas jóvenes masivas en la Nube Menor de Magallanes mediante el código CMFGEN (sección 5.2), y concluyeron que ninguna de las hipótesis mencionadas (con excepción de los rayos X) es suficiente como para dar cuenta del fenómeno de los vientos débiles. En un nuevo estudio basado en el UV y en el óptico de estrellas enanas O galácticas, 
Martins et al. (2005) encuentran discrepancias del orden de 100 en $\dot{M}$ entre los valores medidos y los predichos por la teoría para los tipos espectrales correspondientes a $\log \left(\mathrm{L} / \mathrm{L}_{\odot}\right)<5.2$, concluyendo que la inclusión de rayos $\mathrm{X}$ que afecten la ionización de los vientos es crucial para dar cuenta de las líneas UV en medios de baja densidad. Los autores también encuentran un cambio en la pendiente de la WLR (sección 2.5.1) para las enanas $\mathrm{O}$ tardías y que la metalicidad prácticamente no está relacionada con este problema.

El fenómeno de los vientos débiles también afecta a las velocidades terminales. Martins et al. (2005) encontraron que sus valores caen muy por debajo de los esperados. También sugirieron que el valor de $\alpha$ necesario para reproducir los cocientes $\mathrm{v}_{\infty} / \mathrm{v}_{\text {esc }}$ debería ser del orden de 0.3 , siendo 0.6 el valor aceptado en la teoría estándar. Nuevamente, la presencia de rayos $\mathrm{X}$ ionizantes podrían dar cuenta de este valor del parámetro $\alpha$.

El fenómeno de los vientos débiles aún se encuentra en discusión y tiene implicancias importantes en la evolución de las estrellas masivas. Por ejemplo, una tasa baja de pérdida de masa durante la fase de secuencia principal cambia mucho la evolución posterior de la estrella y las escalas de tiempo evolutivo. También, de acuerdo a Marcolino et al. (2009), la baja pérdida de momento angular a través del viento débil de estas estrellas permitiría que estos objetos lleguen a etapas más evolucionadas con mayores rotaciones (incluso cercanas a la ruptura).

En los últimos años se ha sugerido fuertemente que las determinaciones de $\dot{M}$ mediante observaciones en el UV pueden ser erróneas, ya sea por usar una única línea (doblete C IV $\lambda \lambda$ 1548, 1551), por errores en la abundancia del carbono, o por los cambios mencionados en la ionización debido a los rayos X. Mokiem et al. (2007b) encontraron que, para algunas estrellas, el valor de $\dot{M}$ obtenido a partir de la línea $\mathrm{H} \alpha$ es diferente del medido usando líneas espectrales en el UV, y que podría ser consistente con un viento normal. Esta propuesta ha sido confirmada mediante observaciones directas del espectro en rayos $\mathrm{X}$ de la estrella $\mathrm{O}$ enana de viento débil llamada $\mu \mathrm{Col}$ (Huenemoerder et al. 2012). Las condiciones de un viento normal son detectables sólo en el espectro de rayos X, remarcando la deficiencia de la medida de $\dot{M}$ obtenida en el UV. La medición de frentes de choque (bow-shocks) o esferas de Strömgren en estrellas masivas de alta velocidad (runaway stars) también pone en duda a las mediciones de $\dot{M}$ obtenidas en el UV (Gvaramadze et al. 2012).

\subsubsection{Componentes discretas en absorción}

Uno de los indicadores más claros de la variabilidad del viento, tanto en las estrellas O como en las B supergigantes, es la presencia de componentes discretas en absorción o DACs (discrete absorption componentes). Las DACs son componentes en absorción que aparecen superpuestas a los perfiles de tipo P Cygni, sobre los que se desplazan desde el rojo hacia el azul, en escalas temporales que van desde las horas hasta los días. En la mayoría de los casos, la componente en emisión del perfil P Cygni permanece sin cambios durante toda la migración de las DACs.

Las DACs son observadas en los perfiles de líneas intensas no saturadas de resonancia en el UV, de elementos superionizados tales como el Si IV, N V o el C IV; aunque también pueden aparecen en el visible, por ejemplo en la línea $\mathrm{H} \alpha$ o en He II 
ג4686. Originalmente se las llamaba NACs (componentes delgadas en absorción, narrow absorption components) porque se las suponía como componentes delgadas fijas en la velocidad terminal del viento. Sin embargo, las observaciones de series temporales de los perfiles demostraron que las DACs no permanecen fijas ni siempre son delgadas, sino que migran a través del perfil de la línea. Durante este migración, cuando aparecen son anchas, pero luego se van angostando a medida que aceleran desde el rojo hacia el azul, hasta alcanzar la velocidad terminal, donde aparecen como NACs (Kaper et al. 1997). Por esta razón, se las prefiere denominar como DACs en lugar de NACs (no siempre son delgadas, narrow).

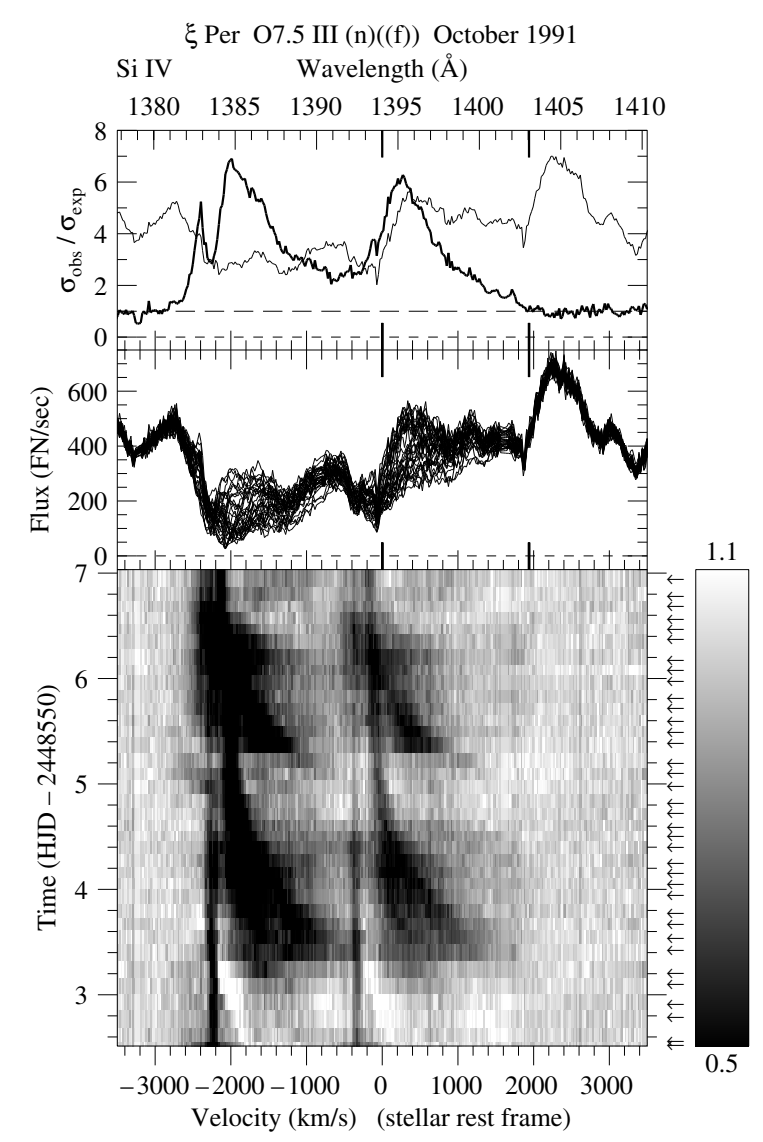

Figura 2.14. La estrella $\xi$ Per muestra DACs en el doblete $\lambda \lambda 1394,1402 \AA$ del Si IV. En el panel superior se muestra el "molde" o templete (línea delgada) del perfil usado para producir el espectro residual, mostrado en el panel inferior. Superpuesto en el panel superior se muestra la amplitud de la variación (línea gruesa). El panel medio presenta una superposición de de los perfiles observados. Se pueden identificar varias DACs, siendo dos las componentes más intensas, separadas en el lapso de dos días. Tomado de Kaper et al. (1997)

Howarth \& Prinja (1989) detectaron DACs en más del $80 \%$ de las estrellas O, sobre una muestra de un total de 203 objetos galácticos. También han sido observadas en estrellas Be (Grady et al. 1987), pero nunca en otra clase de estrellas que no sean supergigantes B. Eso sugiere que, más que un fenómeno general, las DACs son la norma 
entre las estrellas tempranas (Kaper et al. 1997).

Dado que las DACs sólo afectan la componente en absorción de los perfiles, se considera que son variaciones moderadas en la estructura del viento, aunque suficientemente grandes como para cubrir una parte significativa del disco estelar de modo de producir cambios en la absorción. Uno de los factores importantes de las DACs es la recurrencia con que aparecen, estrechamente vinculado con la rotación de la estrella (por ejemplo, Kaper et al. 1996, Arias et al. 2008). Se ha demostrado, además, que sus apariciones están correlacionadas con la variabilidad en $\mathrm{H} \alpha$ (Kaper et al. 1999).

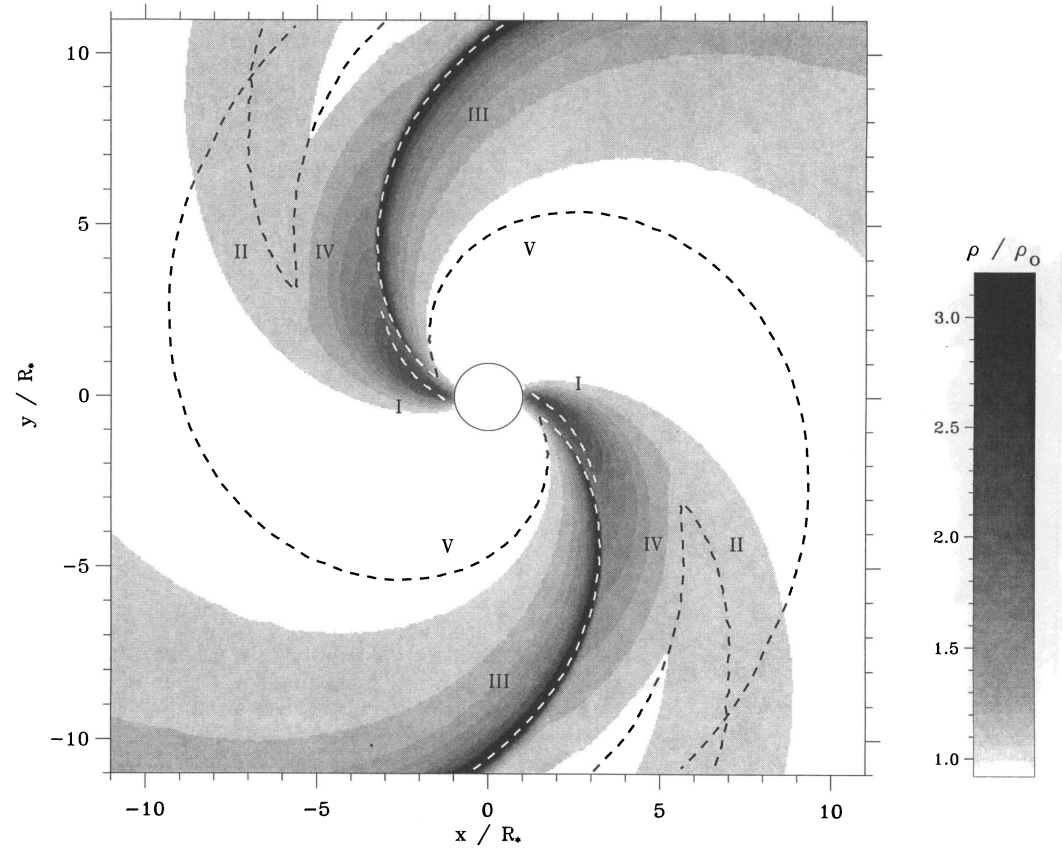

Figura 2.15. En tono de grises, estructura de densidad del modelo CIR. Las líneas a rayas separan las diferentes regiones delimitadas en el modelo. La región $\mathrm{V}$ corresponde a un "quiebre" en la continuidad de la densidad llamado quiebre de onda radiativoacústica de Abbott (radiative-acustic Abbott kink). Tomado de Cranmer \& Owocki (1996)

Varios modelos han tratado de explicar las DACs, pero el más aceptado es el modelo de Regiones Interactuantes de Corrotación o modelo CIR (Corotating Interaction Regions) sugerido por Mullan (1986) y modelado por Cranmer \& Owocki (1996). En este modelo se hipotetiza la presencia de una estructura en el viento estelar producida por la interacción de una corriente rápida y una lenta que se originan en ubicaciones adyacentes sobre la superficie estelar (figura 2.15). Debido a la rotación de la estrella, las corrientes son curvadas, provocando que el material de la corriente rápida colisione con el material lento que está por delante.

Esta estructura da origen a componentes en absorción semejantes a las DACs observadas en las líneas de resonancia en el UV. Sin embargo, no ha sido respondida aún el origen de las corrientes de fluido con diferentes propiedades cinemáticas. Su origen se atribuye a dos posibles causas: las pulsaciones no radiales o los campos magnéticos superficiales. Aunque se sabe que numerosas estrellas tempranas tienen actividad 
pulsacional (por ejemplo, Kraus et al. 2015a), no se conoce un mecanismo consistente para vincular los períodos de ambos fenómenos.

Respecto a los campos magnéticos, a pesar de que las estrellas OB no poseen manto convectivo, podrían existir en ellas campos moderados, probablemente fósiles de la formación estelar. Sin embargo, para estrellas tempranas no peculiares, el campo magnético es débil o actúa a pequeña escala. De acuerdo a Donati et al. (2006), los campos magnéticos no son una característica común de la mayoría de las estrellas tempranas, sino solamente una rara ocurrencia.

\subsubsection{Discordancias en los parámetros del viento}

A pesar de la sofisticación de los modelos de atmósferas actuales, las predicciones de las tasas de pérdida de masa y de las velocidades terminales de las estrellas masivas son todavía inciertas.

Uno de los casos más significativos fue presentado por Fullerton et al. (2006), quienes encontraron una discrepancia en la estimación de $\dot{M}$ en estrellas de tipo O galácticas, basadas en el ajuste de lineas de resonancia del doblete de $\mathrm{P}$ V $\lambda \lambda 1118,1128 \AA$ en el UV observadas con los satélites FUSE, ORFEUS BEFS y Copernicus. La teoría predice que el ion de $\mathrm{P}^{+4}$ es dominante en los vientos de estrellas $\mathrm{O} 7$ a $\mathrm{O} 9.7$, aunque las mediciones indican que el rango más apropiado es O4-O7. En su trabajo, Fullerton et al. (2006) dedujeron que las mediciones de $\dot{M}$ obtenidas a partir de los perfiles de P V son sistemáticamente más bajas que las derivadas de $\mathrm{H} \alpha$ o de la emisión librelibre en radio, las cuales pueden ser mayores en un factor 10 o más. Para conciliar los valores, se propuso que los vientos de las estrellas $\mathrm{O}$ son altamente inhomogéneos, con un importante factor de clumping (sección 2.6.8.

En el rango de las supergigantes B, hay todavía mayores inconsistencias entre las tasas de pérdida de masa observadas y las predichas por los modelos. Vink et al. (2000) presentaron valores teóricos de $\dot{M}$ y una receta para calcularla, para una amplia variedad de estrellas $\mathrm{O}$ y $\mathrm{B}$ galácticas incorporando, en sus modelos, el tratamiento de la dispersión múltiple. Con esta aproximación redujeron la discrepancia para las estrellas O. Sin embargo, como las tasas de pérdida de masa derivadas para las B persisten en su discrepancia, la misma es atribuida a errores sistemáticos en los valores observados.

Crowther et al. (2006) reconocen que los vientos de las estrellas supergigantes B intermedias y tardías son sustancialmente más débiles que los predichos por la teoría estándar, lo cual sería exacerbado si se adicionara clumping (sección 2.6.8) a la región de formación de $\mathrm{H} \alpha$.

Por su parte, Benaglia et al. (2007) encontraron que, aunque los modelos teóricos que estiman $M$ a partir de la emisión en radio y en $\mathrm{H} \alpha$ deberían coincidir, los valores empíricos son, en realidad, diferentes. Los valores medidos de la discrepancia son mayores cuando se consideran objetos por sobre la temperatura correspondiente al salto de bi-estabilidad (sección 2.6.2), mientras que son menores para estrellas con temperaturas más bajas.

Todos estos trabajos concuerdan, como reseña Puls et al. (2008a), en que las tasas de pérdida de masa predichas por la teoría deberían ser menores en un factor de diez. Esta discordancia orientó a los estudios al análisis y la incorporación a los mode- 
los del complejo fenómeno del clumping, en un esfuerzo directo dedicado a reducir la discrepancia en los valores de $\dot{M}$.

Por otro lado, también existe una discrepancia entre los valores empíricos y los teóricos para las velocidades terminales. En las estrellas O, Groenewegen et al. (1989) y Blomme (1990) encontraron que los valores medidos en el UV de $v_{\infty}$ son más bajos que los predichos por el modelo estándar correspondiendo, en el segundo caso, a estrellas O de secuencia principal.

Para las estrellas supergigantes A también se encuentra que las velocidades terminales son menores en un factor tres que las predichas por la teoría Achmad et al. 1997). Para estas estrellas también hay una discrepancia que se observa entre la velocidad terminal y la velocidad de escape, que disminuye para valores mayores de la velocidad de escape, en contradicción con lo estipulado por la teoría (Verdugo et al. 1998). La pendiente negativa en esta relación es atribuida a un cambio en el parámetro $\alpha^{\prime}=\alpha-\delta$, que puede deberse a un cambio en la ionización (a través de $\delta$ ) o a un desacople de los iones del viento (Achmad et al. 1997).

Respecto a la forma de la ley de velocidades a lo largo del viento, la solución CAK tiene la forma de una ley $\beta$ (ecuación 2.2) con $\beta=0.5$, mientras que, para Pauldrach et al. (1986), el valor de este parámetro es $\beta \sim 0.8$ (sección 2.2.10). Sin embargo, en la teoría estándar es común adoptar a esta ley de manera inconsistente. Dados los buenos resultados que ha dado la misma para el ajuste de perfiles en las estrellas más masivas, es habitual hacer uso de la ley $\beta$, extendiendo la potencia $\beta$ a valores muy diferentes de los inferidos en las teorías CAK o m-CAK. De hecho, es común, entre las supergigantes $\mathrm{B}$ intermedias, tardías y las supergigantes $\mathrm{A}$, que se adopten valores de $\beta$ en el rango 1 a 3, con el fin de favorecer el ajuste en la línea $\mathrm{H} \alpha$ (Kudritzki et al. 1999).

Un modelo completamente consistente requeriría que, en lugar de la ley $\beta$ se usaran las soluciones directas de las ecuaciones hidrodinámicas, como se lleva a cabo en este trabajo de tesis.

\subsubsection{Vientos no homogéneos (clumping)}

Los indicadores usados en la estimación de $\dot{M}$ tales como la línea $\mathrm{H} \alpha$ y los excesos en el IR y en radio, están basados en la recombinación de electrones y por lo tanto, dependen de $\rho^{2}$. Esta dependencia hace que sean muy sensibles a pequeños cambios de densidad. Hillier (1991) demostró que la influencia en las alas de los perfiles de línea de la dispersión de los electrones debido a la falta de homogeneidad en la densidad podía reducir, en un factor $\lesssim 2$, la tasa de pérdida de masa en las estrellas Wolf-Rayet (W-R). A partir de ese concepto, se comenzó a estudiar el fenómeno de la no homogéneidad, como una característica estructural importante que podía afectar el diagnóstico de las propiedades de los vientos.

La existencia de concentraciones a pequeña escala en la densidad se denomina clumping, e involucra un medio donde la materia se concentra en pequeños grumos, dejando casi vacío el espacio entre los mismos. Tal ausencia de homogeneidad es esperable ya que los vientos impulsados por radiación son inestables (sección 2.6.1). Owocki et al. (1988); Feldmeier (1995); Dessart \& Owocki (2005) modelaron las inestabilidades y encontraron que la sucesión de velocidades que crecen y decrecen produce fuertes ondas 
de choque invertidas (reverse shocks) donde el material se comprime en regiones densas espacialmente reducidas, separadas por grandes zonas con densidades mucho más bajas (ver figura 2.10).

Los indicios espectrales de esta ausencia de homogeneidad en el viento de las estrellas $\mathrm{OB}$ son numerosos. Si bien, las primeras evidencias fueron encontradas en los vientos de las estrellas WR, en subestructuras variables en emisión superpuestas a los perfiles de líneas espectrales (Moffat et al. 1988), Eversberg et al. (1998) y, posteriormente, Lépine \& Moffat (2008) las observaron también en estrellas O (figura 2.16), sugiriendo que este fenómeno era usual en los vientos de estrellas tempranas.
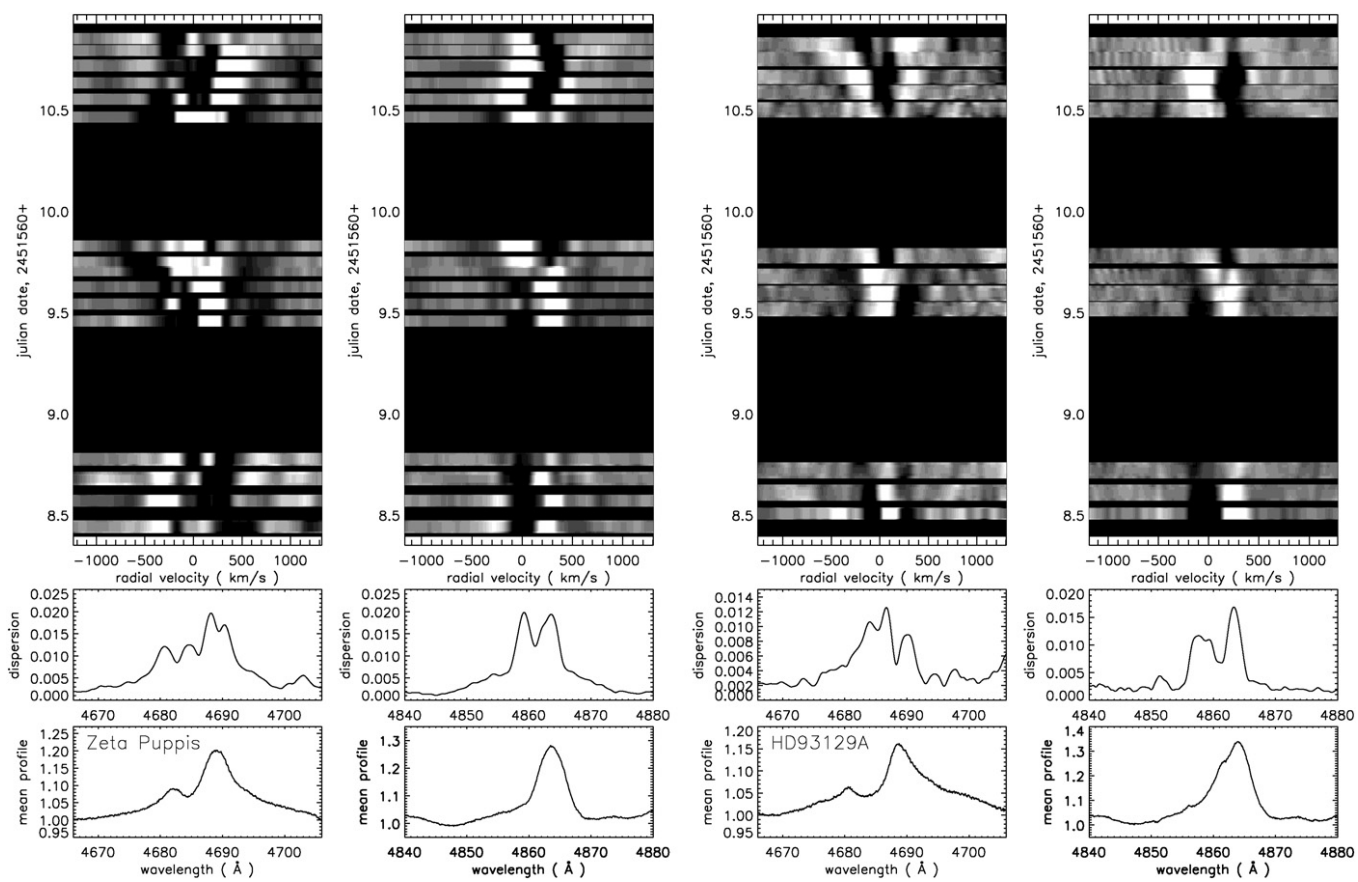

Figura 2.16. Series temporales de los residuos que resultan de restar, a cada observación, un perfil medio de la línea de He II 4686, para las estrellas Of $\zeta$ Puppis (izquierda) y HD 93129A (derecha). Las variaciones de estos residuos son atribuidos a la observación directa del fenómeno de clumping. Tomado de Lépine \& Moffat (2008)

Otra evidencia surge de la observación de las componentes en absorción saturadas (black troughs) de los perfiles P Cygni en el UV (panel izquierdo de la figura 1.10). Lucy (1982) sugirió que estas características eran un indicio de campos de velocidades no monótonos, que producen una importante dispersión hacia atrás de los fotones (back-scattering). Esto muestra que el fenómeno del clumping también afecta la ley de velocidades.

La existencia de las líneas de emisión en rayos X observadas con los satélites XMMNewton y CHANDRA, han sido interpretadas como el producto de vientos confinados magnéticamente o shocks embebidos en el viento, originados por las inestabilidades de los vientos impulsados por radiación en líneas o por colisiones entre los clumps. En algunos casos es posible estudiar el grado de inohomogeneidad o la formación de 'grumos' (clumping), su distribución radial y la forma de los clumps, a partir de la 
observación de estas líneas (Puls et al. 2008b).

Para incorporar el clumping a los códigos de atmósfera, la suposición estándar es que los grumos son ópticamente delgados, mientras que el espacio intermedio está vacío. El exceso de densidad interior en los grumos es descripto por el llamado factor de clumping, $\mathrm{f}_{\mathrm{cl}}$, que se define como:

$$
f_{\mathrm{cl}}=\frac{\left\langle\rho^{2}\right\rangle}{\langle\rho\rangle^{2}},
$$

donde $\langle\rho\rangle$ es la densidad media del gas (Sundqvist et al. 2012).

La consecuencia más importante de incorporar este fenómeno es que los valores de $\dot{M}$ derivados de $\mathrm{H} \alpha$ o radio , cuya dependencia con $\rho$ es cuadrática, y fueran calculados sin tener en cuenta este efecto, deberían considerarse como sobrevaluados en un factor $\sqrt{f_{\mathrm{cl}}}$, por lo cual la tasa de pérdida de masa verdadera debería ser consistentemente menor. Siguiendo esta aproximación, se han derivado factores de clumping comprendidos entre 10 y 50, con clumping localizado en la base del viento (por ejemplo, Bouret et al. 2005), lo cual reduce a los valores de $\dot{M}$ en factores comprendidos entre 3 y 7 . Puls et al. (2006) estudiaron la estratificación radial del clumping a partir del modelado simultáneo multifrecuencia de observaciones, encontrando que, en los vientos densos, el clumping es más fuerte en la parte interna del viento, por factores comprendidos entre 4 y 6, y que las tasas de pérdida de masa calculadas sin clumping, deben reducirse en factores de, al menos, 2 a 3. Mediante un análisis en las líneas FUV de P V en $\lambda \lambda 1118$, $1128 \AA$, Fullerton et al. (2006) encontraron que el factor debería ser entre 10 o 100, en comparación con los valores obtenidos a partir de líneas de recombinación sin clumping. Por esta razón, en algunos trabajos se optó por reducir las tasas de pérdida de masa con el objeto de debilitar las líneas de resonancia en el UV, mientras que la emisión en $\mathrm{H} \alpha$ se mantiene acorde a los valores observados, adoptando para esto factores de clumping muy altos (Bouret et al. 2012).

Si los grumos (clumps) no son ópticamente delgados, se deben tener en cuenta otros efectos importantes en el cálculo del transporte de radiación. Los clumps producen una bloqueo de la radiación en su interior pero, a su vez, se incrementa el escape de la radiación entre los mismos, lo cual se llama porosidad o macro-clumping (Feldmeier et al. 2003, Owocki et al. 2004). En este caso, despreciar la porosidad, lleva a una subestimación de la tasa de pérdida de masa (Oskinova et al.2007). Estos investigadores proponen que $\mathrm{H} \alpha$ no está afectada por la porosidad, mientras que el doblete resonante de P V resulta significativamente debilitado por este efecto. Este hecho sugiere que es necesario incorporar clumps ópticamente gruesos para la evaluación adecuada de los parámetros del viento. Šurlan et al. (2013) incorporó estos efectos en el código PoWR, empleando un método Monte-Carlo 3D en estrellas O, resolviendo la discrepancia entre las tasas de pérdida de masa calculadas con $\mathrm{H} \alpha$ y con las líneas de $\mathrm{P}$ V.

En vientos con mucha aceleración, los fotones de una línea espectral pueden iteractuar solamente en una región muy delgada, debido al ancho Doppler del perfil de la línea. Esto posibilita que los fotones se escapen del viento, por medio de los canales o poros, en el espacio de velocidad. Este efecto se conoce como porosidad en velocidad o vorosidad (vorosity) y fue discutido por Owocki (2008). Esta propiedad puede estimarse con el factor de llenado de la velocidad, dado por: 


$$
f_{\mathrm{vel}}=\frac{\delta v}{\Delta v}
$$

donde $\delta v$ es el corrimiento en velocidad dentro del clump y $\Delta v$ es la separación en velocidad entre los clumps. La fuga de fotones en el espacio de velocidades produce el mismo efecto que la porosidad espacial, es decir, reduce la gravedad efectiva como en el caso de clumps ópticamente delgados (Sundqvist et al. 2012).

Prinja \& Massa (2010), por medio del análisis de líneas de resonancia de Si IV en el UV (las líneas de resonancia no son afectadas por los clumps ópticamente delgados), encontraron evidencias empíricas de la existencia de grumos ópticamente gruesos en el viento de supergigantes B. Las mediciones de los perfiles, efectuadas sobre una extensa muestra de estrellas, concuerda consistentemente con los modelos de vorosidad de Sundqvist et al. (2011). El efecto de la vorosidad atenúa los cambios en la pérdida de masa producidos por los clumps, acercando los valores de $\dot{M}$, a los valores clásicos CAK. 


\section{Capítulo 3}

\section{Vientos en rotación impulsados por radiación}

Resumen del capítulo: Se introducen los vientos en rotación impulsados por radiación. Se resumen las ecuaciones básicas para estos vientos en el plano del ecuador estelar, junto con las relaciones de escala obtenidas para tasas de pérdida de masa y velocidades terminales. Se presentan las soluciones de las ecuaciones hidrodinámicas: las soluciones rápidas y las soluciones $\Omega_{\text {lenta }} y \delta_{\text {lenta }}$, obtenidas por Curé. Se sintetizan las principales aplicaciones de estas soluciones a objetos astronómicos. Finalmente, se explican las características básicas del código de cálculo hidrodinámico HYDWIND.

\subsection{Propiedades de los vientos impulsados por ra- diación en rotación}

Como se mencionó en la sección 1.2.3, las estrellas OB pueden tener velocidades de rotación moderadas o rápidas. Eso significa que la rotación puede afectar, sensiblemente, tanto la física como el diagnóstico de los parámetros de los vientos impulsados por radiación. De acuerdo a Puls et al. (2008b), la influencia de la rotación sobre el viento puede agruparse en los siguientes efectos principales:

- La rotación modifica la dinámica propia del viento.

- Los apartamientos de la simetría esférica resultante en los altos rotadores, pueden afectar las determinaciones de los parámetros del viento, especialmente $\dot{M}$.

- Puede aparecer variabilidad en los perfiles de línea debido a la interacción entre las peculiaridades fotosféricas (manchas, pulsaciones), con la rotación y la dinámica del viento. Un ejemplo de esta interacción es el modelo de Regiones de Interacción Corrotantes (co-rotating interaction regions) o modelo CIR (sección 2.6.6).

Los primeros modelos que incorporaron la rotación a la teoría de vientos impulsados por radiación fueron presentados por Marlborough \& Zamir (1975), que consideraron solamente la fuerza de radiación en el continuo. Castor (1979) propuso un modelo con 
velocidad latitudinal nula, para el cual el momento angular por unidad de masa es conservado. En este modelo se incluyó el término de la fuerza centrífuga en la ecuación de movimiento, prediciendo una tasa de pérdida de masa con pocas modificaciones debidas a la rotación pero obteniendo, a la vez, un gradiente de velocidades considerablemente más bajo, con una velocidad terminal menor que en el caso no rotante. Castor notó que el punto singular se encuentra considerablemente más apartado de la superficie estelar que en el caso de rotación nula.

Marlborough \& Zamir (1984) describieron la solución de un viento rotante isotermo, obteniendo considerables apartamientos de la teoría CAK cuando se adopta una baja temperatura cinética a lo largo del viento. Las mayores diferencias respecto a los resultados de la teoría CAK, fueron un decrecimiento más lento de la densidad con la distancia y una velocidad terminal más baja. Los apartamientos de la simetría esférica estimados por Marlborough \& Zamir (1984) sugirieron, de manera cualitativa, que el viento podría formar una estructura de tipo discoidal, afín a los modelos propuestos para explicar las observaciones de las estrellas Be.

Mediante modelos simples, Friend \& Abbott (1986) y Pauldrach et al. (1986) confirmaron que la inclusión de la rotación tiende a disminuir la velocidad terminal del viento e incrementar la tasa de pérdida de masa.

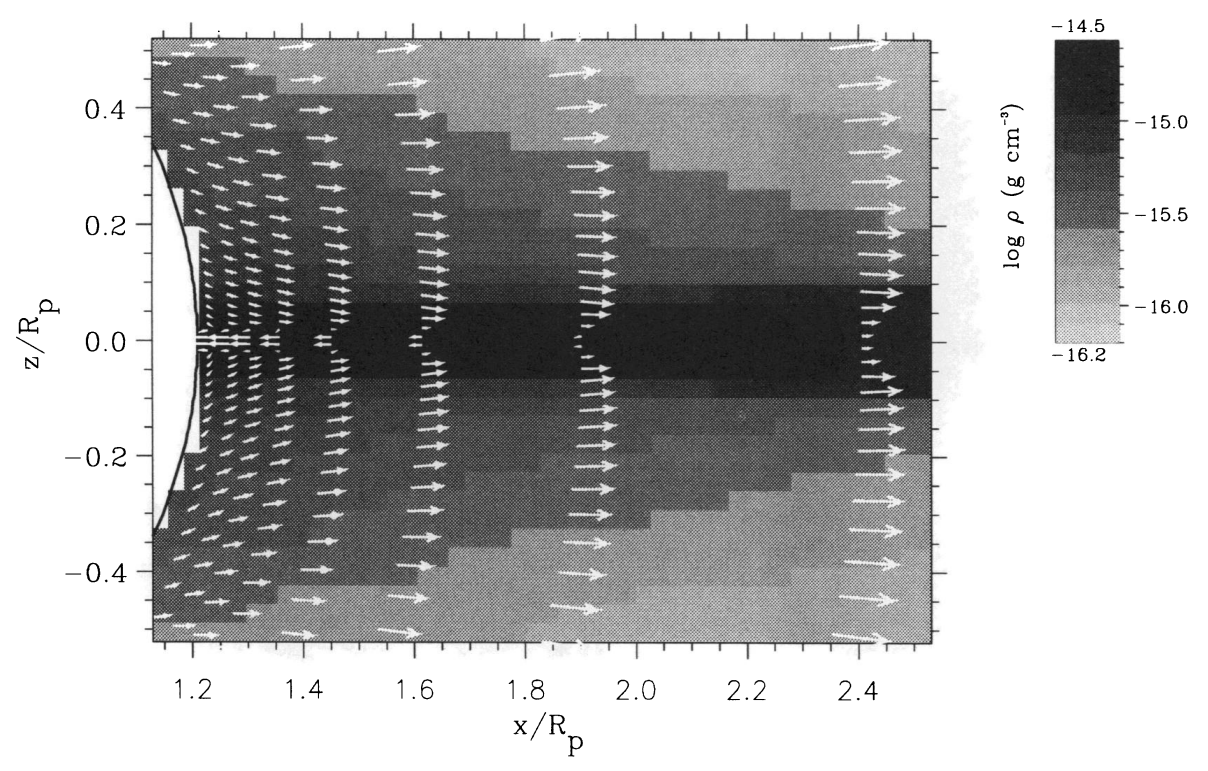

Figura 3.1. Modelo de disco comprimido por el viento o WCD (wind compressed disk) en la región ecuatorial interna. La densidad del material se indica con escala de grises. Cada flecha muestra la magnitud y la dirección de la velocidad del flujo. Nótese la fuerte concentración de gas en la zona interna del disco. Tomado de Owocki et al. (1994).

Uno de los avances que tuvo más repercusión en la teoría de vientos en rotación impulsados por radiación fue el desarrollo del modelo 2-D llamado modelo de "disco comprimido por el viento" (wind-compressed disk model) o modelo WCD, propuesto por Bjorkman \& Cassinelli (1993) y probado en simulaciones numéricas por Owocki et al. (1994). En estos trabajos se propuso que la formación de discos alrededor de 
estrellas rápidamente rotantes como las estrellas Be, puede deberse a la existencia de corrientes meridionales que concentran el material hacia el ecuador (figura 3.1).

Sin embargo, poco tiempo después de la presentación del modelo WCD, Owocki et al. (1996) demostraron que la influencia de fuerzas de radiación no radiales podrían inhibir la formación de tales discos (en el trabajo de Bjorkman \& Cassinelli (1993), todas las fuerzas eran consideradas como fuerzas centrales), a pesar de que las mismas podían llegar a tener una magnitud menor que el $10 \%$ de la fuerza de radiación radial.

Curé (2004) y Curé et al. (2011) produjeron importantes innovaciones en la teoría de los vientos impulsados por radiación en estrellas rotantes. Sus modelos dieron lugar a la distinción de las tres clases de soluciones hidrodinámicas: la solución rápida (que es la solución estándar m-CAK), la solución $\Omega_{\text {lenta }}$, que surge para altos rotadores, y la solución $\delta_{\text {lenta }}$, que está ligada a la ionización del material. Estas soluciones son explicadas con más detalle en las siguientes secciones.

\subsection{Ecuaciones hidrodinámicas para vientos en ro- tación}

Las ecuaciones básicas para el flujo 3-D estacionario de una estrella rotante fueron presentadas por Marlborough \& Zamir (1975) y Marlborough \& Zamir (1984). Para plantear estas ecuaciones se supone un sistema de coordenadas esférico $(\mathrm{r}, \theta, \phi)$ tal que el punto $\mathrm{r}=0$ corresponde a la posición del centro del sistema, $\theta=\pi / 2$ es el plano ecuatorial, y $\phi$ es el ángulo acimutal, medido en el mismo sentido que la rotación estelar (figura 3.2. El viento es descripto mediante las componentes de la velocidad $\left(v_{r}, v_{\theta}, v_{\phi}\right)$ según las coordenadas $(\mathrm{r}, \theta, \phi)$, respectivamente.

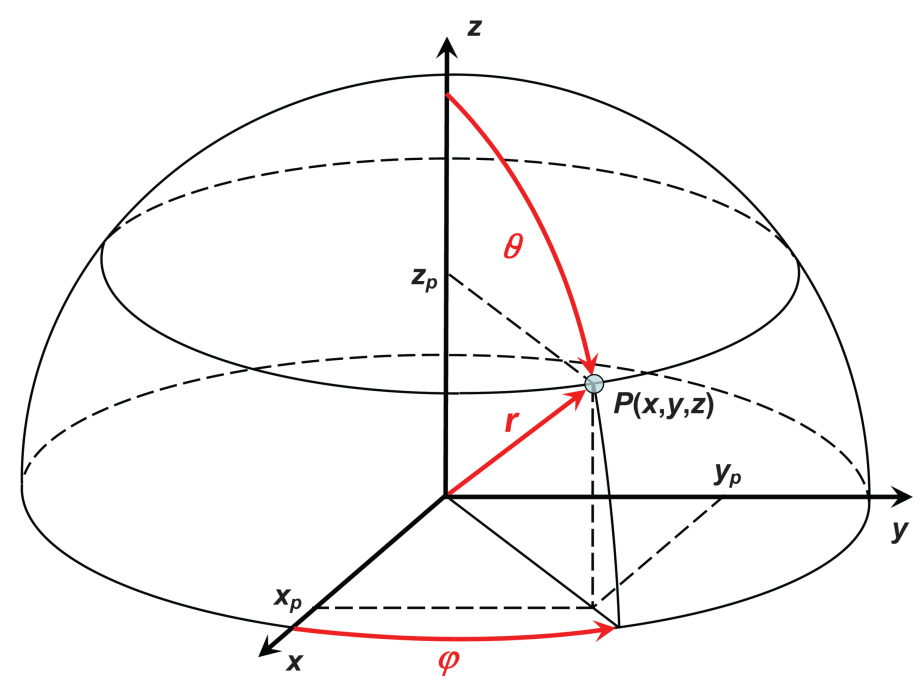

Figura 3.2. Sistema de coordenadas empleado en las ecuaciones hidrodinámicas generales para un viento impulsado por radiación con rotación.

En esta configuración, el campo de velocidades es simétrico respecto al eje de rotación (eje z), en relación al plano ecuatorial. Si la presión del gas es isotrópica y se 
desprecian la viscosidad y cualquier campo magnético, las ecuaciones que describen el viento son:

$$
\begin{aligned}
\frac{1}{r^{2}} \frac{\partial}{\partial r}\left(r^{2} \rho v_{r}\right)+\frac{1}{r \sin \theta} \frac{\partial}{\partial \theta}\left(\rho v_{\theta} \sin \theta\right) & =0 \\
v_{r} \frac{\partial v_{r}}{\partial r}+\frac{v_{\theta}}{r} \frac{\partial v_{r}}{\partial \theta}-\frac{v_{\theta}^{2}+v_{\phi}^{2}}{r}+\frac{1}{\rho} \frac{\partial p}{\partial r} & =f_{r}, \\
v_{r} \frac{\partial v_{\theta}}{\partial r}+\frac{v_{\theta}}{r} \frac{\partial v_{\theta}}{\partial \theta}+\frac{v_{r} v_{\theta}}{r}-\frac{v_{\phi}^{2} \cot \theta}{r}+\frac{1}{\rho r} \frac{\partial p}{\partial \theta} & =f_{\theta}, \\
v_{r} \frac{\partial v_{\phi}}{\partial r}+\frac{v_{\theta}}{r} \frac{\partial v_{\phi}}{\partial \theta}+\frac{v_{r} v_{\phi}}{r}+v_{\theta} v_{\phi} \frac{\cot \theta}{r} & =f_{\phi},
\end{aligned}
$$

donde $\rho$ es la densidad de masa, $p$ es la presión del gas y $f_{r}, f_{\theta}$ y $f_{\phi}$ son las componentes de la fuerza externa total por unidad de masa en las direcciones r, $\theta$ y $\phi$, respectivamente. Las fuerzas externas comprenden, en este caso, a la fuerza gravitatoria y a las fuerzas de radiación. La primera de las ecuaciones 3.1 es la ecuación de continuidad, derivada de la ecuación 2.3 para el sistema en consideración.

Estas ecuaciones deben completarse con una ecuación de conservación de la energía, obtenida a partir de la ecuación 2.19. En este caso, la misma puede escribirse como:

$$
v_{r} \frac{\partial E}{\partial r}+\frac{v_{\theta}}{r} \frac{\partial E}{\partial \theta}+p v_{r} \frac{\partial}{\partial r}\left(\frac{1}{\rho}\right)+p \frac{v_{\theta}}{r} \frac{\partial}{\partial \theta}\left(\frac{1}{\rho}\right)=4 \pi \int_{0}^{\infty} \kappa_{\nu}\left(J_{\nu}-S_{\nu}\right) d \nu
$$

donde $E$ es la energía interna por unidad de masa del gas, $\kappa_{\nu}$ es el coeficiente de absorción, $J_{\nu}$ es la intensidad media del campo de radiación y $S_{\nu}$, la función fuente. Esta última ecuación resulta muy compleja de tratar ya que, para evaluar su solución, hace falta resolver, de manera acoplada, el transporte de radiación. Sólo de esta manera se podrían obtener la intensidad media y de la función fuente, simultáneamente con las variables hidrodinámicas del caso. Pero aunque fuera posible hacerlo, la presencia de otras fuentes de calentamiento del gas, diferentes a la propia radiación estelar (por ejemplo, a través de las ondas de choque), haría que esta ecuación no esté completa y sus resultados podrían ser inciertos. Por esos motivos, la ecuación de la energía es habitualmente reemplazada por una ley de temperaturas simple, por ejemplo, una ley de gas isotermo.

Las estrellas con rotación moderada o alta pueden presentar grandes apartamientos de la simetría esférica. Esos apartamientos deberían ser mayores en el plano ecuatorial, donde los efectos dinámicos producidos por la rotación podrían ser significativos. Por lo tanto, resulta importante estudiar, especialmente, el comportamiento del flujo en el plano ecuatorial $\theta=\pi / 2$.

$\mathrm{Si}$ el sistema se limita al plano del ecuador, la velocidad $v_{\theta}=0 \mathrm{y}$ ninguna de las componentes de las velocidades tendrá variaciones respecto a la coordenada $\theta$ :

$$
\frac{\partial v_{r}}{\partial \theta}=\frac{\partial v_{\phi}}{\partial \theta}=\frac{\partial v_{\theta}}{\partial \theta}=0
$$

Por lo tanto, las ecuaciones en el plano ecuatorial se simplifican como: 


$$
\begin{aligned}
\frac{d}{d r}\left(r^{2} \rho v_{r}\right) & =0, \\
v_{r} \frac{d v_{r}}{d r}-\frac{v_{\phi}^{2}}{r}+\frac{1}{\rho} \frac{d p}{d r} & =f_{r}, \\
v_{r} \frac{d v_{\phi}}{d r}+\frac{v_{r} v_{\phi}}{r} & =f_{\phi},
\end{aligned}
$$

donde se han reemplazado las derivadas parciales por derivadas totales ya que, en el plano ecuatorial, solamente se mantiene la dependencia con respecto a $r$. Por otra parte se llamará directamente $v$ a $v_{r}$, eliminando el subíndice de la proyección radial del vector velocidad, mientras que se mantendrá la notación de $v_{\phi}$ para la velocidad acimutal.

La primera ecuación del sistema 3.4 es, nuevamente, la ecuación de continuidad, que puede integrarse como se hizo para obtener la ecuación 2.7, para dar en este caso:

$$
F_{m}=4 \pi r^{2} \rho v=\text { constante, }
$$

donde $F_{m}$ es el flujo de masa local expulsado en el plano ecuatorial. Si bien este valor es diferente al de la tasa de pérdida de masa total $\dot{M}$ para un sistema $3-D$, en esta tesis se lo considerará como representativo (proporcional) del valor total para el viento estelar. Debido a que la fuerza centrífuga es máxima para $\theta=\pi / 2$, es decir, en el plano ecuatorial, puede esperarse que la parte más densa del viento corresponda a este plano.

En la última ecuación del sistema 3.4 , se supone que la fuerza según la coordenada $\phi$ es nula, es decir, $f_{\phi}=0$. Por lo tanto esa ecuación queda independiente de $v_{r}$ y puede integrarse inmediatamente, dando:

$$
v_{\phi}=D r^{-1}
$$

donde $D$ es una constante arbitraria de integración. Esta ecuación expresa la conservación del momento angular. En el caso en que $r=R_{*}$, entonces $v_{\phi}=v_{\text {rot }}$, que es la velocidad fotosférica de rotación. En tal caso se puede evaluar el valor de la constante $D$, resultando $D=v_{\text {rot }} R_{*}$. Entonces, la expresión para la velocidad acimutal (ecuación 3.6), dependiente de la distancia al centro de la estrella, queda como:

$$
v_{\phi}=v_{\text {rot }} \frac{R_{*}}{r} .
$$

La segunda ecuación del sistema 3.4 es la ecuación de momento del viento. Por lo tanto, para estimar la influencia de la rotación sobre el viento en el plano ecuatorial, se debe modificar la ecuación de momento (ecuación 2.12), incorporando un término correspondiente a la fuerza centrífuga.

De este modo, el término de la fuerza centrífuga tiene una dependencia con la distancia de $r^{-3}$, quedando de la forma:

$$
v_{\phi}^{2} / r=v_{\text {rot }}^{2} R_{*}^{2} / r^{3} .
$$

Finalmente, la ecuación a resolver es:

$$
v \frac{d v}{d r}=-\frac{1}{\rho} \frac{d p}{d r}-\frac{G M_{*}(1-\Gamma)}{r^{2}}+\frac{v_{\phi}^{2}(r)}{r}+g^{L}\left(\rho, \frac{d v}{d r}, n_{e}\right),
$$


en la cual todos los términos, exceptuando el que corresponde a la fuerza centrífuga, tienen el significado ya presentado en el capítulo 2.

Habitualmente, la velocidad de rotación se expresa en términos de una cantidad adimensional llamada tasa rotacional $\Omega$, que es definida como:

$$
\Omega=v_{\text {rot }} / v_{\text {break }},
$$

que toma valores comprendidos entre 0 y 1 . La velocidad de ruptura $v_{\text {break }}$ es la velocidad para la cual se igualan la fuerza centrífuga y la fuerza gravitatoria (corregida por la fuerza de radiación del continuo):

$$
v_{\text {break }}=\sqrt{\frac{G M_{*}(1-\Gamma)}{R_{*}}}=\frac{v_{\text {esc }}}{\sqrt{2}},
$$

en la cual, la última igualdad surge de considerar la ecuación 2.88 . El cociente $\Omega$ es evaluado en $\mathrm{r}=\mathrm{R}_{*}$, donde $\mathrm{R}_{*}$ es el radio correspondiente a $\tau=2 / 3$.

Conservando la parametrización estándar m-CAK, la fuerza de línea de la ecuación de movimiento 3.9 tiene la forma dada por la ecuación 2.79, que se repite aquí para facilitar el seguimiento de los cálculos:

$$
g^{L}\left(\rho, \frac{d v}{d r}, n_{e}\right)=\frac{C}{r^{2}} C F\left(r, v, \frac{d v}{d r}\right)\left(r^{2} v \frac{d v}{d r}\right)^{\alpha}\left(\frac{n_{e}}{W(r)}\right)^{\delta} .
$$

donde $C F\left(r, v, \frac{d v}{d r}\right)$ es el factor de corrección por disco finito de la estrella presentado en la sección 2.2.8. Nuevamente, los parámetros de la fuerza de radiación son los introducidos por Castor et al. (1975) y Abbott (1982b): $k, \alpha$ y $\delta$. La constante:

$$
C=\Gamma G M_{*} k\left(\frac{4 \pi}{\sigma_{e} v_{t h} \dot{M}}\right)^{\alpha},
$$

contiene la tasa de pérdida de masa y es el autovalor.

\subsubsection{Relaciones de escala para vientos en rotación}

De acuerdo a la dependencia con la coordenada $r$ del término de la fuerza centrífuga (ecuación 3.8) en la ecuación de momento (ecuación 3.9), Puls et al. (1999) propusieron nuevas relaciones de escala, semejantes a las presentadas en la sección 2.5.1 pero, en este caso, para vientos impulsados por radiación de estrellas tempranas en rotación. El término de la fuerza centrífuga puede unirse al término de la fuerza gravitatoria, para generar una gravedad efectiva, de la forma:

$$
g_{\mathrm{eff}}=\frac{G M_{*}(1-\Gamma)}{r^{2}}\left(1-\frac{\Omega^{2} R_{*}}{r}\right) .
$$

Entonces, pueden usarse las relaciones encontradas para vientos impulsados por radiación (sección 2.5.1) incorporando una masa modificada en un factor semejante a $\left(1-\Omega^{2}\right)$. En tal caso, la relación de escala para la tasa de pérdida de masa (ecuación 2.105) se transforma como: 


$$
\dot{M}(\Omega)=\dot{M}(\Omega=0)\left(1-\Omega^{2}\right)^{1-1 / \alpha^{\prime}}
$$

recordando que $\alpha^{\prime}=\alpha-\delta$. Esta relación es válida si el punto crítico de la ecuación de movimiento, que determina el valor de $\dot{M}$, permanece cercano a la superficie estelar. En el caso de la velocidad terminal, puede extenderse la relación 2.104, con el mismo criterio:

$$
v_{\infty}(\Omega)=v_{\infty}(\Omega=0)\left(1-\Omega^{2}\right)^{1 / 2}
$$

La dependencia de estas relaciones con la tasa rotacional $\Omega$ pueden verse en la figura 3.3 .

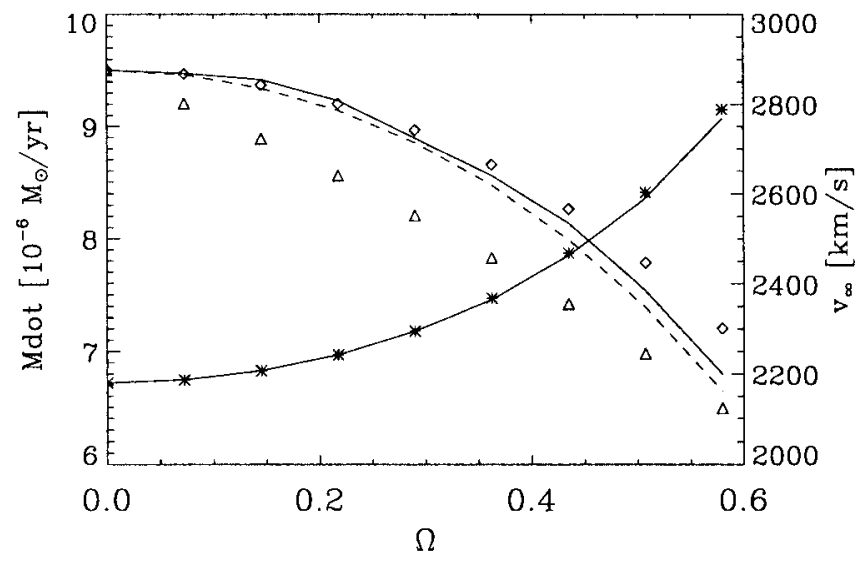

Figura 3.3. Relaciones de escala para $\dot{M}$ y $\mathrm{v}_{\infty}$ en el plano ecuatorial de un viento impulsado por radiación que emerge de una estrella en rotación. Se indica con líneas llenas, la solución hidrodinámica completa encontrada por Pauldrach et al. (1986). Se indica con asteriscos la relación 3.13 para $\dot{M}$. Respecto a $\mathrm{v}_{\infty}$, se indica con línea a rayas una relación de escala más detallada donde se considera la influencia decreciente con la distancia de la fuerza centrífuga, con diamantes la relación dada por la ecuación 3.14 con $\beta=0.8$, y con triángulos, una relación de escala dada por Friend \& Abbott (1986). Tomado de Puls et al. (1999)

\subsection{Soluciones hidrodinámicas para vientos en ro- tación}

Para resolver la ecuación de momento (ecuación 3.9), Curé (2004) usó una metodología semejante a la desarrollada en la sección 2.2.10, con la que se derivó la solución de vientos no rotantes impulsados por radiación. Para esto propuso el siguiente cambio 
de variables:

$$
\begin{aligned}
u & =-\frac{R_{*}}{r}, \\
w & =\frac{v}{a}, \\
w^{\prime} & =\frac{d v}{d u},
\end{aligned}
$$

donde $a$ es la velocidad isotérmica del sonido (de la relación $p=a^{2} \rho$, ecuación 2.15).

De esta manera, las ecuaciones 3.9 y 3.5, junto a la forma de la fuerza de radiación (ecuación 2.80), se transforman en:

$$
\left(1-\frac{1}{w^{2}}\right) w \frac{d w}{d u}+A+\frac{2}{u}+a_{\mathrm{rot}}^{2} u-C^{\prime} C F g(u)(w)^{-\delta}\left(w \frac{d w}{d u}\right)^{\alpha}=0,
$$

donde:

$$
\begin{aligned}
A & =\frac{G M(1-\Gamma)}{a^{2} R_{*}}=\frac{v_{\mathrm{esc}}^{2}}{2 a^{2}}, \\
C^{\prime} & =C\left(\frac{\dot{M} D}{2 \pi} \frac{10^{-11}}{a R_{*}^{2}}\right)^{\delta}\left(a^{2} R_{*}\right)^{(\alpha-1)}, \\
g(u) & =\left(\frac{u^{2}}{1-\sqrt{1-u^{2}}}\right)^{\delta}, \\
a_{\mathrm{rot}} & =\frac{v_{\mathrm{rot}}}{a}, \\
D & =\frac{1+Z_{\mathrm{He}} Y_{\mathrm{He}}}{1+A_{\mathrm{He}} Y_{\mathrm{He}}} \frac{1}{m_{H}},
\end{aligned}
$$

en las que $v_{\text {esc }}$ es la velocidad de escape (ecuación 2.88), $Y_{\mathrm{He}}$ es la abundancia del helio relativa al hidrógeno, $Z_{\mathrm{He}}$ es la cantidad de electrones libres que provee el helio, $A_{\mathrm{He}}$ es el número másico del helio y $m_{H}$ es la masa del protón.

La ecuación 3.16 tiene una forma del tipo $F\left(u, w, w^{\prime}\right)=0$ y es una ecuación diferencial no lineal. Para resolverla y obtener su autovalor $C^{\prime}$, se requiere que la solución pase (y verifique las condiciones necesarias) por un punto crítico y que, además, cumpla una condición de borde en el límite interno de la atmósfera. Para esta última condición, puede haber dos opciones:

$$
\int_{R_{*}}^{\infty} \sigma_{e} \rho(r) d r=\frac{2}{3}
$$

que fija el valor de la profundidad óptica en la región más profunda de la fotosfera en 2/3, o:

$$
\rho\left(R_{*}\right)=\rho_{*},
$$


donde $\rho_{*}$ es un valor asignado a la densidad en la fotosfera, correspondiente a la distancia del centro de la estrella que se adopta como radio estelar. En general, su valor es del orden de $10^{-11} \mathrm{~g} \mathrm{~cm}^{-3}$. La opción más adecuada es utilizar el valor de densidad apropiado, tomado de un modelo de atmósfera como, por ejemplo, los modelos de Kurucz (1979).

Para ubicar el punto crítico, se requiere la condición de singularidad:

$$
\frac{\partial}{\partial w^{\prime}} F\left(u, w, w^{\prime}\right)=0
$$

en donde se debe cumplir la condición de regularidad:

$$
\frac{d}{d u} F\left(u, w, w^{\prime}\right)=\frac{\partial F}{\partial u}+\frac{\partial F}{\partial w} w^{\prime}=0 .
$$

Las últimas dos ecuaciones deben ser válidas en la localización del punto crítico.

\subsubsection{La solución rápida o m-CAK}

Para obtener la solución de las ecuaciones planteadas, Curé (2004) introdujo una nueva transformación de coordenadas en las ecuaciones 3.16, 3.20 y 3.21 de la forma:

$$
\begin{aligned}
& Y=w w^{\prime} \\
& Z=\frac{w}{w^{\prime}},
\end{aligned}
$$

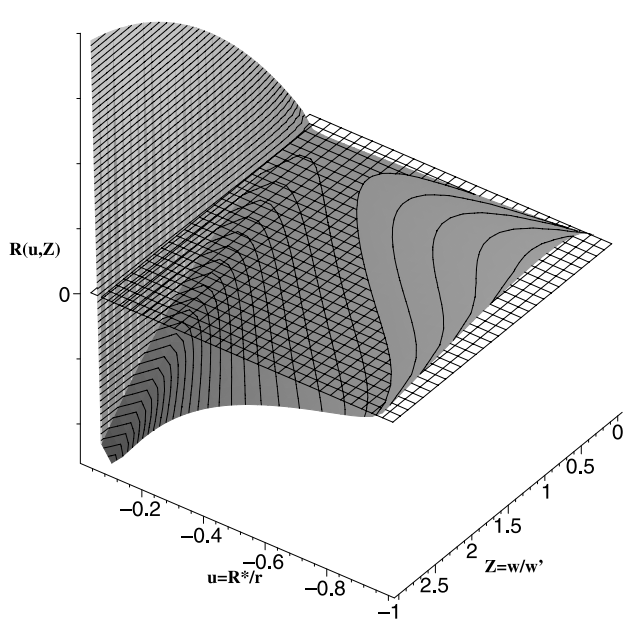

(a)

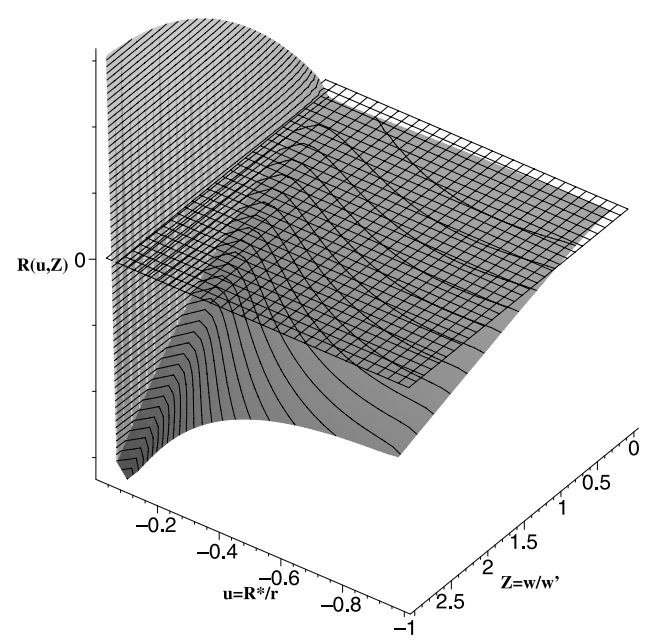

(b)

Figura 3.4. a) Intersección de la superficie $\mathrm{R}(\mathrm{u}, \mathrm{Z})$ (calculada para los parámetros de una estrella $\mathrm{O} 5 \mathrm{~V}$ con $\Omega=0.5$ ) con el plano $\mathrm{R}(\mathrm{u}, \mathrm{Z})=0$, delimitando las curvas correspondientes a las familias de soluciones que satisfacen simultáneamente la ecuación de momento y las condiciones de singularidad y regularidad. b) Para alta rotación, no hay intersección entre las superficies para soluciones tipo m-CAK. Tomado de Curé (2004). 
generando tres nuevas ecuaciones con cuatro incógnitas: $u, C^{\prime}, Y$ y $Z$. Aunque este sistema no puede resolverse por sí mismo, es posible, relacionando sus ecuaciones, obtener una función $R(u, Z)$ cuyas raíces son los puntos críticos por donde deben pasar las soluciones (para más detalles, ver Curé 2004). La función $R(u, Z)$ es una superficie, cuya intersección con el plano $R(u, Z)=0$ origina dos curvas que se corresponden con dos familias de puntos críticos, como se observa en la figura $3.4(\mathrm{a})$.

\begin{tabular}{ccccc}
\hline \hline$\Omega$ & $\beta=0.8$ & $\beta=1$ & $\beta=2$ & Numérica \\
\hline 0.0 & 1.026 & 1.034 & 1.084 & 1.033 \\
0.3 & 1.027 & 1.035 & 1.088 & 1.036 \\
0.5 & 1.029 & 1.038 & 1.098 & 1.040 \\
0.7 & 1.036 & 1.048 & 1.129 & 1.051 \\
\hline
\end{tabular}

Tabla 3.1. Ubicación del punto crítico para diferentes valores de la tasa rotacional $\Omega$. En las columnas 2, 3 y 4, se detalla la posición del punto crítico para diferentes valores de $\beta$ en una solución de tipo m-CAK. En la columna 5 , se indican los valores obtenidos numéricamente por Curé (2004). Tomado de Curé (2004).

Una de las intersecciones entre la superficie $R(u, Z)$ y el plano $R(u, Z)=0$ corresponde a la solución clásica m-CAK o solución rápida. Cada solución de viento de tipo m-CAK se origina en la superficie estelar bajo la condición de borde elegida (ecuación 3.18 ó 3.19 y luego se desarrolla hacia afuera, pasando por uno de los puntos críticos de esta curva.

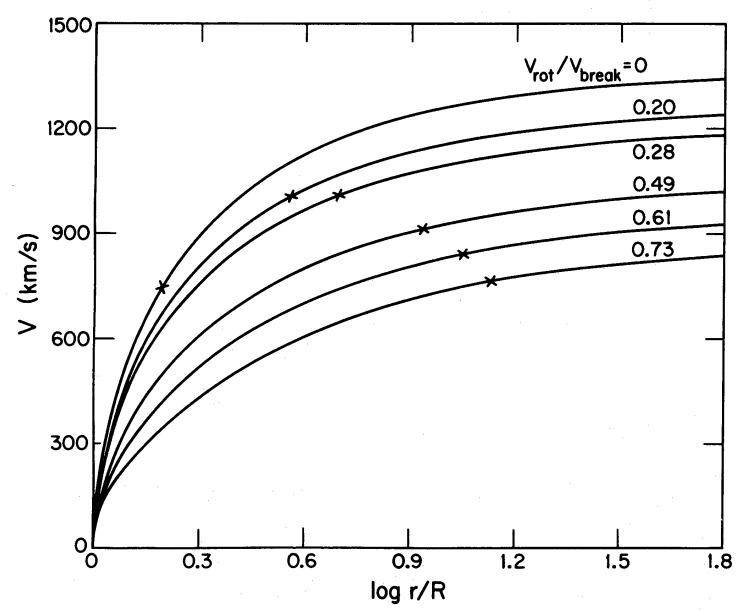

Figura 3.5. Leyes de velocidad para varios modelos rotantes, cada uno indicado con el valor de $\Omega$. Las cruces marcan la ubicación del punto crítico. Tomado de Friend \& Abbott (1986).

La tabla 3.1 compara las ubicaciones de los puntos críticos para vientos de una estrella de tipo $\mathrm{O} 5 \mathrm{~V}$ en rotación, correspondientes a las soluciones que se obtienen directamente de las ecuaciones hidrodinámicas, y los valores derivados en modelos mCAK con leyes $\beta$ de velocidades. Los cálculos numéricos muestran la mayor coincidencia 
con el modelo m-CAK con $\beta=1$. El corrimiento del punto crítico en la dirección del flujo al aumentar la velocidad de rotación, puede observarse en la figura 3.5. También, en el mismo gráfico, puede verse que es notorio el decremento en todo el perfil de velocidades $\mathrm{y}$, consecuentemente, en el valor de $\mathrm{v}_{\infty}$, al aumentar la velocidad de rotación.

Usando las expresiones para la aceleración en las líneas espectrales dada por Villata (1992), y la metodología propuesta por Müller \& Vink (2008), Araya et al. (2014) presentaron expresiones analíticas útiles para modelar las soluciones hidrodinámicas rápidas, de modo que el uso de las mismas no implique la resolución completa de las ecuaciones ni la disponibilidad de los códigos de cálculo necesarios.

\subsubsection{La solución $\Omega_{\text {lenta }}$}

Si la velocidad de rotación es muy alta $(\Omega=0.9$ para una estrella de tipo O5 V), la superficie $R(u, Z)$ definida en la sección 3.3.1, se hace negativa en la región de la solución rápida, y no hay intersección con el plano $R(u, Z)=0$, tal como se ve en la figura 3.4(b). En esa situación no habría puntos críticos. De esta manera, se demuestra que no hay solución m-CAK para estrellas con alta rotación, explicando la razón por la cual muchos autores reportaron problemas numéricos para encontrar la ubicación de puntos críticos en estas condiciones (por ejemplo, Friend \& Abbott 1986; de Araujo et al. 1994).

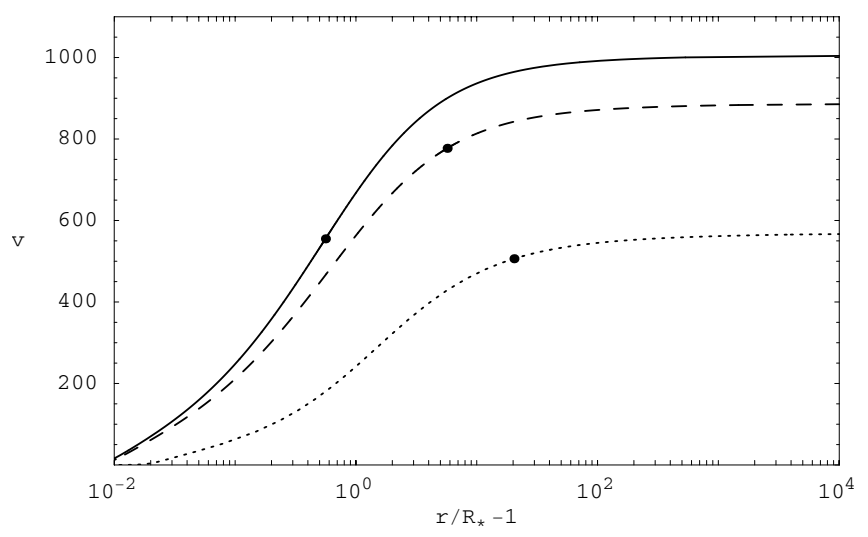

Figura 3.6. Leyes de velocidad en función de $r / R_{*}-1$, para un modelo sin rotación ( $\Omega=0$, línea continua), para una solución rápida $(\Omega=0.25$, línea a rayas) y para una solución $\Omega_{\text {lenta }}(\Omega=0.8$, línea punteada). Nótese el decremento importante en la velocidad terminal, en comparación con los modelos m-CAK y el desplazamiento del punto crítico, que sigue la tendencia observada en la figura 3.5. Tomado de Curé \& Rial (2004).

Sin embargo, aún subsiste una familia de puntos críticos, que corresponde a una solución con valores de $u$ pequeños $(u \leq-2$ en la figura 3.4(b) . La solución que pasa por los puntos críticos delimitados por esta curva es la solución $\Omega_{\text {lenta. Esta solución }}$ aparece para valores de $\Omega>0.7$, es decir para objetos con alta rotación, y fue descubierta por Curé (2004). 
La solución $\Omega_{\text {lenta }}$ predice velocidades terminales bajas (generalmente menores que $400 \mathrm{~km} \mathrm{~s}^{-1}$ ), dependiendo de los parámetros fundamentales de la estrella, y este viento es más denso que el correspondiente a la solución rápida o m-CAK. Para lograr una representación aproximada a estas soluciones con leyes de velocidad de tipo $\beta$, se requeriría que $\beta>1$. Un ejemplo de la ley de velocidad para la solución $\Omega_{\text {lenta }}$ puede verse en la figura 3.6 .
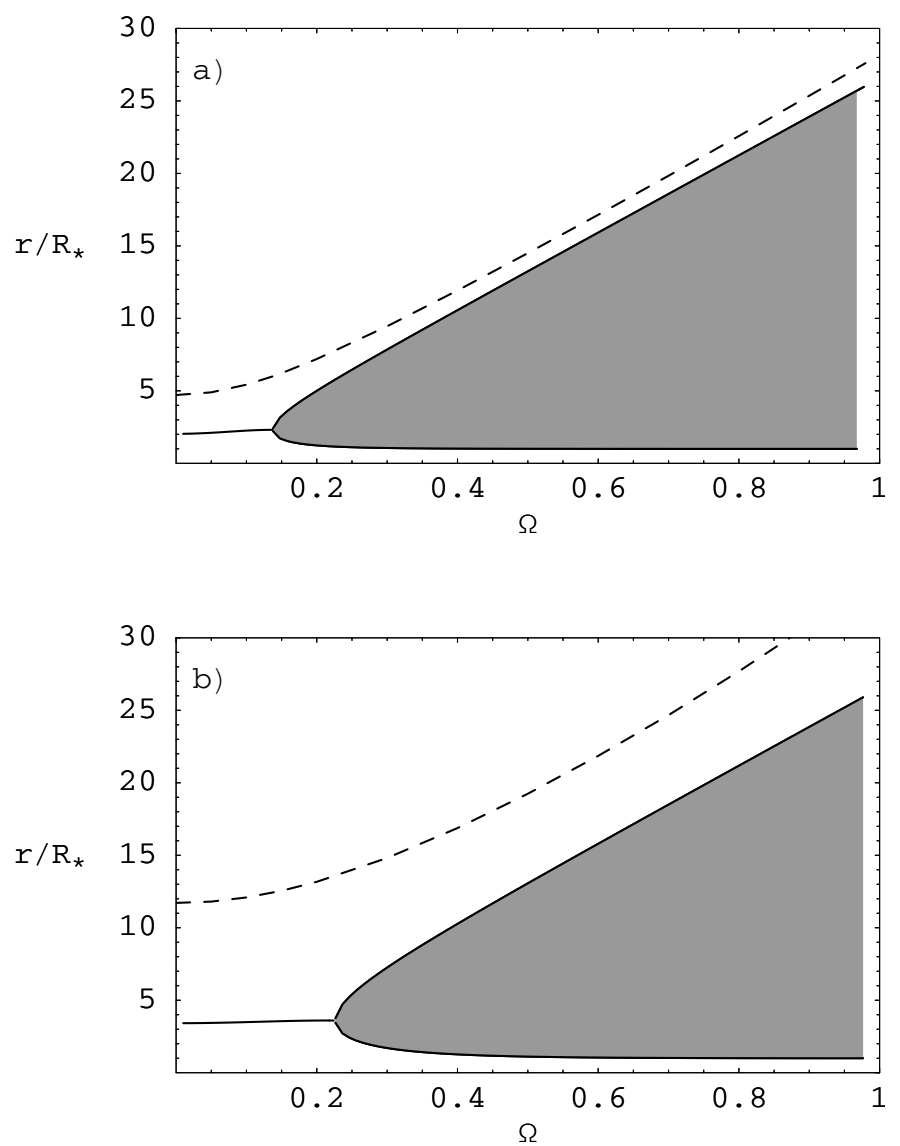

Figura 3.7. En este gráfico se muestra, en línea continua, los límites superior e inferior para las ubicaciones posibles de los puntos críticos en las soluciones de vientos en rotación impulsados por radiación. La región gris corresponde a una zona "prohibida" donde no puede haber puntos críticos. La línea a trazos corresponde a la ubicación de los puntos críticos para el caso en que se usa la condición de borde sobre la profundidad óptica en la fotosfera (ecuación 3.18). El caso a) muestra el análisis topológico para un parámetro $\delta=0.02$, mientras que, para el caso b), $\delta=0.1$. Tomado de Curé \& Rial (2004).

Curé \& Rial (2004) llevaron a cabo un exhaustivo análisis topológico de la solución

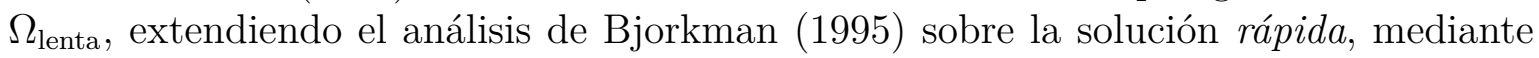
la incorporación de la rotación y la ionización del viento. En ese trabajo, los autores confirmaron que la rotación desplaza la ubicación del punto singular de la solución rápida hacia regiones más alejadas de la superficie estelar (figura 3.6). Para un viento 
sin ionización (frozen in ionization), es decir con el parámetro $\delta=0$, dedujeron que sólo existe la solución rápida con un punto singular tipo "X". Si $\delta$ no es nulo, aparece un segundo punto singular de tipo "atractor" más cercano a la superficie estelar.

Como se ve en la figura 3.7, que corresponde al análisis topológico de las soluciones hidrodinámicas, la existencia de los puntos críticos correspondientes a las soluciones está fuertemente influido por la tasa rotacional $\Omega$. Para valores bajos de $\Omega$, hay una única solución con un único punto crítico. Al aumentar la velocidad de rotación, las soluciones posibles se bifurcan dando, simultáneamente, soluciones de tipo rápida u

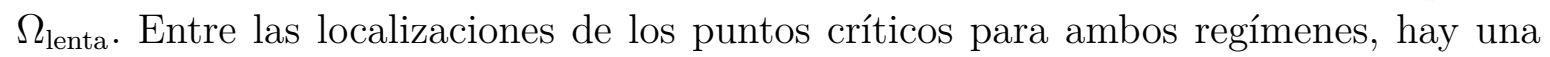
región para la cual no existen soluciones, ya que no puede determinarse allí un punto crítico. También puede apreciarse en la figura, el alejamiento del punto crítico de la fotosfera estelar para el caso de la solución rápida (rama superior en la bifurcación). Se debe tener en cuenta que este análisis fue realizado por Curé \& Rial (2004) para valores bajos del parámetro $\delta$ (no mayores a 0.1 ) solamente.

\subsubsection{Aplicaciones de la solución $\Omega_{\text {lenta }}$}

Como se detalló en la sección 3.3.2, la solución $\Omega_{\text {lenta }}$ sólo existe para modelos con valores de $\Omega>0.7$, es decir, objetos con alta rotación. Por esta razón, las primeras aplicaciones de esta solución fueron sugeridas para modelar estrellas de alta rotación tales como las estrellas Be (Curé 2004).

Las estrellas Be son estrellas de tipo espectral B, casi todas pertenecientes a la secuencia principal, con alta rotación y líneas en emisión en el óptico e IR cercano, especialmente en $\mathrm{H} \alpha$ y otros miembros de la serie de Balmer, He I y varias líneas de metales. Todos estos fenómenos son atribuidos a la presencia de un disco ecuatorial de gas libre de polvo, geometricamente delgado, que se mueve en rotación kepleriana y que se difunde lentamente (Rivinius et al. 2013). Curé (2004) propuso un modelo para las estrellas Be consistente en un viento polar poco denso y veloz, representado por la solución rápida (región de rotación nula), y un viento lento y denso para dar cuenta del disco ecuatorial de materia en la región de alta rotación correspondiente a las bajas latitudes estelares. En ese trabajo, Curé obtuvo un contraste de densidad entre las regiones ecuatoriales y polares $\left(\rho_{\text {ecu }} / \rho_{\text {pol }}\right)$ del orden de 100 , aunque sólo en regiones más próximas a la estrella, siendo 10 el valor de esta magnitud para el resto del viento. De acuerdo a la interpretación usual de las observaciones, el valor de 100 para el contraste de densidad debería mantenerse a lo largo de mayores distancias de la superficie estelar que lo estimado.

Los efectos combinados de la rotación rápida y los cambios en los parámetros de la fuerza de radiación generados por el mecanismo de bi-estabilidad entre las regiones polares y las ecuatoriales de una estrella en rápida rotación, fueron estudiados por Curé et al. (2005). En este caso, los autores buscaron dar cuenta de los contrastes de densidad propios de las estrellas $\mathrm{B}[\mathrm{e}]$, modelando las mismas con velocidades altas de rotación $\left(v_{\text {rot }} \sim 200 \mathrm{~km} \mathrm{~s}^{-1}\right.$, lo que equivale a $\left.\Omega \gtrsim 0.6\right)$. Con estos modelos obtuvieron contrastes de densidad del orden de $10^{2}$ a $10^{4}$ para las regiones cercanas a la superficie estelar (figura 3.8), y valores entre $10^{1}$ y $10^{2}$ para radios mayores. Estos valores están en buen acuerdo con los contrastes observados, los cuales son del orden de $10^{2}-10^{3}$ (Zickgraf et al. 1989; Bjorkman 1998). 


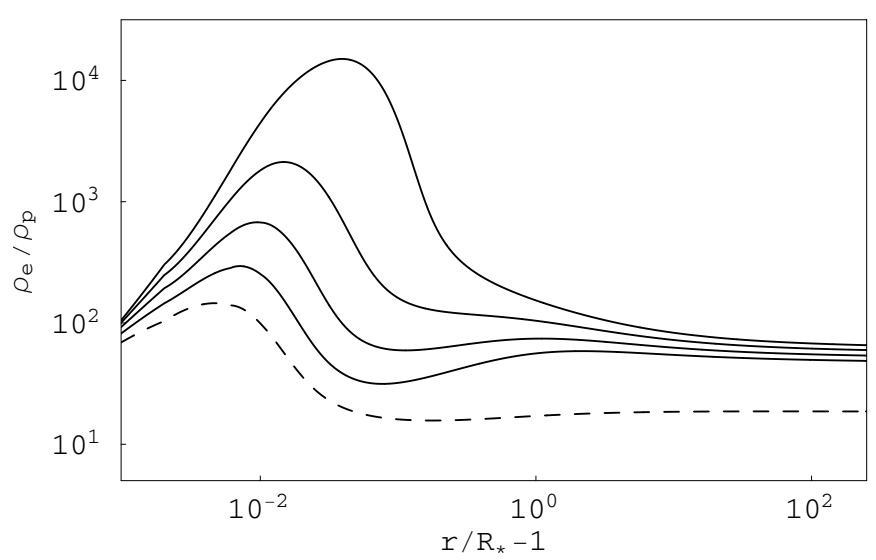

Figura 3.8. Contrastes de densidad en función de $r / R_{*}-1$. La línea a rayas corresponde a $\Omega=0.6$, las líneas continuas corresponden a los modelos con $\Omega=0.7,0.8,0.9,0.99$. A mayor valor de $\Omega$, mayor contraste de densidad. Tomado de Curé et al. (2005).

Madura et al. (2007) verificaron la existencia de la solución $\Omega_{\text {lenta }}$ por medio de modelos 1D en analogía con la eyección de gases en una tobera (nozzle). Para rotaciones bajas, sus modelos generaron un punto crítico próximo a la superficie estelar, que se corresponde con un máximo posible en la tasa de pérdida de masa, recuperando la solución de tipo CAK. Sin embargo, para una estrella con una rotación mayor en un $75 \%$ del valor para la rotación crítica, sus modelos generaron un flujo ecuatorial más denso y lento similar a la solución encontrada por Curé.

Combinando estudios analíticos y simulaciones hidrodinámicas, Madura et al. (2007) determinaron que, en el límite de rotación de $\Omega \approx 0.745$, se produce un cambio de una aceleración rapidamente creciente a una suavemente creciente. Los autores concluyeron que ese cambio se debe a una sobrecarga en la pérdida de masa, por encima del valor límite que puede permitir un flujo monotonamente creciente. El valor de ese límite corresponde a la tasa de pérdida de masa para un modelo CAK de estrella puntual. Cuando la rotación se hace muy alta, la pérdida de masa supera este monto, y el viento "salta" a una nueva solución más suave, en una nueva solución con un flujo subcrítico.

Por otro lado, Madura y sus colaboradores encontraron una "zona gris" en el dominio de las soluciones, comprendida entre las tasas rotacionales que van entre $\Omega \approx$ $0.745 \mathrm{y} \sim 0.85$, donde hay dos soluciones diferentes posibles. En este dominio de las soluciones, se espera un quiebre o "kink" marcando una discontinuidad en la pendiente de la velocidad (figura 3.9). En estos casos el estado es estacionario es decir, independiente del tiempo, a pesar de la presencia de la discontinuidad. Si la tasa rotacional es 0.85, la sobrecarga en el viento se vuelve muy grande y el material se acumula hasta un cierto radio. En tal situación, los modelos predicen un frente de "re-acreción" o caída de material hacia la estrella. Ese retorno del flujo, si la condición de borde interna de la simulación lo permite, reestablece un viento con baja aceleración, es decir, instala una solución en el régimen $\Omega_{\text {lento }}$. Para tasas rotacionales mayores a 0.86 , el flujo es subcrítico (aunque supersónico), por lo cual la única solución posible es la lenta, con una tasa de pérdida de masa que ha saturado el valor límite de estrella puntual CAK. 


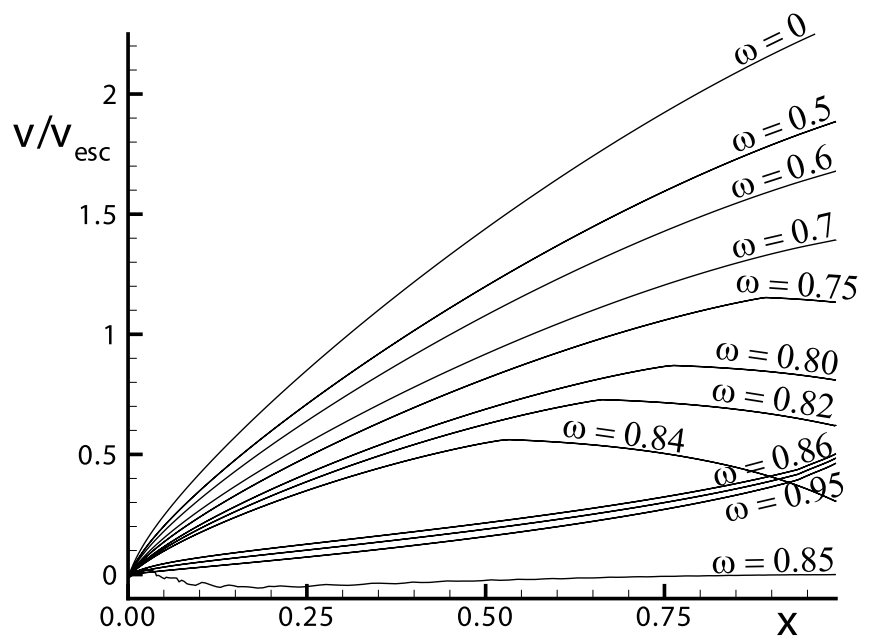

Figura 3.9. Gráfico de la velocidad del flujo respecto a la velocidad de escape $\left(v / v_{\text {esc }}\right.$ en función de la coordenada $x=1-R_{*} / r$, para modelos con diferentes valores de $\Omega$ (indicado como $\omega$ ). Nótese el quiebre o kink para las soluciones con $\Omega=0.75,0.8$, 0.82, 0.84 y la solución colapsada para 0.85 . Para valores mayores, se genera el régimen lento. Tomado de Madura et al. (2007).

La idea de modelar las estrellas Be con la solución $\Omega_{\text {lenta }}$ fue revisitada por Silaj et al. (2014), quienes obtuvieron velocidades terminales, tasas de pérdida de masa y contrastes de densidad, en buen acuerdo con las observaciones de estrellas Be, mediante variaciones de los parámetros $\alpha$ y $k$. Los modelos más adecuados implicaron valores bajos de $\alpha$ y altos de $k$.

\subsubsection{La solución $\delta_{\text {lenta }}$}

El hallazgo de la solución $\Omega_{\text {lenta }}$ despertó el interés por la búsqueda de nuevas soluciones de las ecuaciones hidrodinámicas, explorando modelos con baja rotación pero con valores no convencionales para los parámetros de la fuerza de radiación. De esta manera, Curé et al. (2011) encontraron una nueva familia de soluciones, que aparece cuando se adoptan, para el parámetro $\delta$, valores mayores que los usados habitualmente en la teoría estándar, y que existe aún en el caso de vientos no rotantes $(\Omega=0)$. A esta solución se la llamó solución $\delta_{\text {lenta }}$.

Si los valores de los gradientes de ionización son grandes, se espera que el parámetro $\delta$ adopte valores altos, mayores a $\delta=1 / 3$ que corresponde a un medio con hidrógeno neutro como elemento de traza (Puls et al. 2000). Estas configuraciones de alta ionización podrían tener lugar en vientos inestables con flujos de radiación adicionales al estelar (ver, por ejemplo, la sección 2.6.1).

La nueva solución fue aplicada a las estrellas supergigantes A, cuyas velocidades de rotación son bajas $\left(v_{\text {rot }} \sim 40 \mathrm{~km} \mathrm{~s}^{-1} ; \Omega<0.4\right)$, como así también son bajos los valores de sus velocidades terminales. En su trabajo, Curé et al. (2011) probaron algunos conjuntos de parámetros $k, \alpha$ y $\delta$, en arreglos diferentes a los comúnmente utilizados en la teoría estándar. Para $\alpha$, los dos valores adoptados fueron 0.49 y 0.59 , mientras 
que para $k$, fueron 0.37 y 0.86 . Particularmente, para $\delta$, los valores probados están en el rango comprendido entre 0 (frozen in ionization) y 0.5 contemplando, de este modo, estructuras alternativas en la ionización a lo largo del viento. Las tasas de rotación probadas correspondieron a los valores de $\Omega=0$ y 0.4 .

Cuando Curé y sus colaboradores usaron valores del parámetro $\delta \lesssim 0.25$, obtuvieron soluciones rápidas con $v_{\infty}$ del orden de $500 \mathrm{~km} \mathrm{~s}^{-1}$ o mayores. Sin embargo, cuando probaron con valores de $\delta \gtrsim 0.3$, obtuvieron la solución $\delta_{\text {lenta }}$.

Esta nueva solución está caracterizada por sus campos de velocidades con valores de $v_{\infty}$ comprendidos entre 150 y $250 \mathrm{~km} \mathrm{~s}^{-1}$ y tasas de pérdida de masa entre $10^{-6}$ y $10^{-10} M_{\odot}$ año ${ }^{-1}$. Los autores reportaron que no fue posible encontrar soluciones en el intervalo $0.22<\delta<0.30$. Los incrementos en los valores de $\delta$ para los modelos produjeron un aumento simultáneo de la velocidad terminal y de la tasa de pérdida de masa.

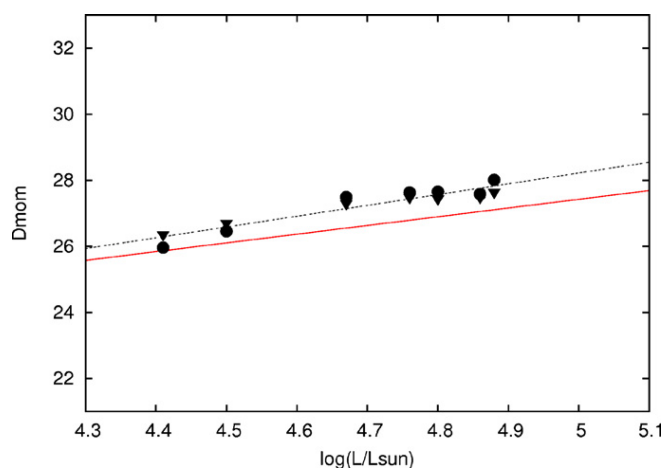

(a)

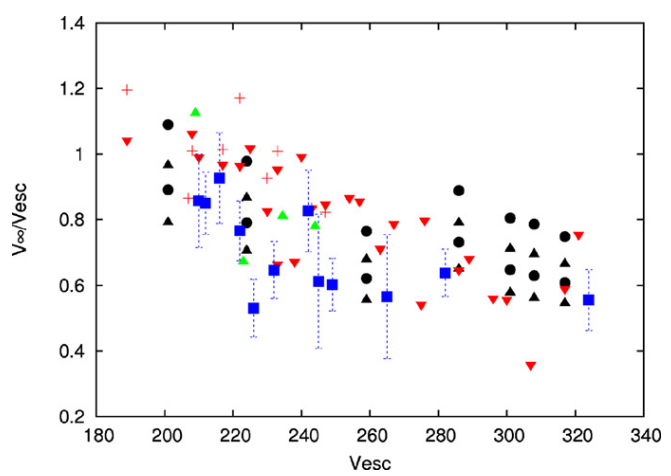

(b)

Figura 3.10. a) Comparación entre la relación Momento del Viento - Luminosidad (WLR) teórica (línea sólida) y empírica (línea punteada), junto a valores obtenidos para la solución $\delta_{\text {lenta }}$ para una modelo con $\Omega=0.4$, con diferentes valores de $\alpha$ y $k$ (triángulos y círculos). b) Relación entre $v_{\infty} / v_{\text {esc }}$ versus $v_{\text {esc }}$ para soluciones rápidas (círculos llenos) y soluciones lentas (triángulos llenos) Los demás símbolos indican mediciones de estos valores a partir de perfiles de línea P Cygni saturados o por componentes discretas en absorción o DACs, entre otros. Tomado de Curé et al. (2011).

La figura 3.10(a) muestra que los resultados de Curé et al. (2011) se ajustan adecuadamente a la relación WLR (ver sección 2.5.1) para las estrellas supergigantes A. Además, en ese trabajo, se analizó la tendencia de $v_{\infty} / v_{\text {esc }}$ en función de $v_{\text {esc }}$ (figura 3.10(b), obteniendo concordancia con el decaimiento citado por Verdugo et al. (1998) para esta clase de estrellas. Este resultado está en contraposición con lo establecido por la teoría estándar (ver la sección 2.6.7).

\subsubsection{El código HYDWIND}

Michel Curé, junto a sus colaboradores en la Universidad de Valparaíso en Chile, ha desarrollado un código de cálculo que permite encontrar las soluciones hidrodinámicas de la ecuación 3.9, siguiendo la metodología planteada por Curé (2004) (sección 3.3). Este código se llama HYDWIND (en el trabajo de Silaj et al.(2014) recibió por primera 
vez esa denominación) y permite obtener tanto las soluciones rápidas como las $\Omega_{\text {lenta }}$ y $\delta_{\text {lenta }}$, dependiendo de los valores suministrados para los parámetros $k, \alpha$ y $\delta$.

Para la ejecución del código, se requiere el ingreso de los parámetros fundamentales de la estrella: la temperatura efectiva $\left(\mathrm{T}_{\text {eff }}\right)$, el logaritmo de la gravedad superficial $(\log \mathrm{g})$, el radio estelar en unidades del radio solar $\left(R_{*}\right)$ y la tasa de rotación $(\Omega)$. Junto a éstos se deben ingresar también, los valores correspondientes a los parámetros de la fuerza de radiación $k, \alpha$ y $\delta$. Por último, se debe optar por una condición de borde interna para la solución buscada, ya sea dada por la ecuación 3.18 o la ecuación 3.19.

Para localizar al punto crítico, a partir del cual se integra la ecuación de momento, el código hace uso de una ley de velocidades de prueba que, en este caso, es una ley $\beta$ de velocidades (ecuación 2.2). Para esto, se debe ingresar también un valor inicial del parámetro $\beta$, un valor de prueba de la velocidad terminal y un valor tentativo para el autovalor. La solución obtenida es de forma numérica.

La solución a las ecuaciones hidrodinámicas que se obtienen como salida de HYDWIND consisten, esencialmente, en los valores de $\dot{M}, v_{\infty}$ y las leyes de velocidades y de densidades.

HYDWIND es un código ágil que demora menos de un minuto en ejecutarse, en un procesador I5. Sin embargo, en algunas ocasiones, si los valores de la función de prueba no son los adecuados o no se obtiene convergencia, el código puede finalizar con una solución errónea discontinua que se debe controlar. Para superar este inconveniente, se debe recurrir a un proceso de prueba y error, que puede llevar horas, para obtener un modelo correctamente convergido.

En esta tesis se utiliza el código HYDWIND para obtener los modelos hidrodinámicos de viento, manteniendo la condición de borde que requiere que la profundidad óptica en la fotosfera sea $\tau_{0}=2 / 3$ (ecuación 3.18). Esa condición se adopta en todos los modelos para uniformizar los cálculos y evitar que la elección de un valor arbitrario para la densidad $\rho$ en la base de la fotosfera, condicione las propiedades de los vientos modelados. Se busca que, con esta elección de la condición de borde, se evite una dependencia con el modelo de fotósfera. 
3. Vientos en rotación impulsados por radiación 


\section{Capítulo 4}

\section{Dominios de las soluciones de vientos en rotación impulsados por radiación}

Resumen del capítulo: Se analizan las propiedades de las tres soluciones: rápida, $\Omega_{\text {lenta }} y \delta_{\text {lenta }}$, en base a su dependencia con los parámetros de la fuerza de radiación y los dominios que los mismos ocupan en el espacio de parámetros. Se estudian los límites entre cada región, con especial atención a la brecha que separa las soluciones rápidas de las lentas. Todos los contenidos presentados en este capítulo han sido desarrollados por el autor como parte de este trabajo de Tesis.

\subsection{Análisis del dominio de las soluciones hidrodi- námicas}

En su trabajo original, Curé et al. (2011) no exploraron completamente el dominio de la solución $\delta_{\text {lenta }}$, es decir, no delimitaron las regiones del espacio de parámetros para las cuales se establece ya sea el régimen rápido o el régimen lento. En ese trabajo, los autores indagaron algunos modelos aislados, con reducidas combinaciones de los parámetros involucrados, describiendo las soluciones originadas para sólo dos valores de la tasa de rotación y un reducido conjunto de parámetros de la fuerza de radiación. Por otro lado, sus esfuerzos se concentraron a una estrecha gama de parámetros fundamentales estelares (tipos espectrales, masas y radios estelares, metalicidades, etc.), acotando considerablemente los escenarios físicos de las estrellas en consideración.

De la misma manera, en los trabajos referidos a la solución $\Omega_{\text {lenta }}$ Curé 2004; Madura et al. 2007), sólo se estableció un valor de referencia para la tasa rotacional (en general, $\Omega>0.7)$ para separar las condiciones bajo las cuales domina la solución rápida o la

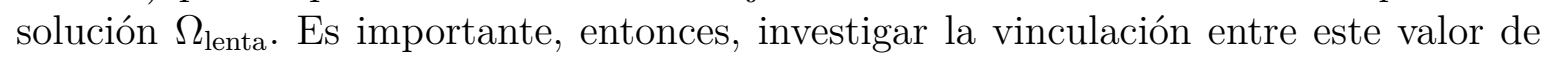
$\Omega$ con otros parámetros relevantes tales como la temperatura efectiva o la gravedad superficial. Además, tampoco ha quedado establecido un límite, en el espacio de los parámetros, entre ambas soluciones lentas, $\Omega_{\text {lenta }} \mathrm{y} \delta_{\text {lenta }}$.

Por estas razones, aún restan por investigar muchos tópicos importantes respecto 
a las soluciones hidrodinámicas estacionarias, particularmente, sobre las condiciones físicas específicas que establecen a cada clase de solución como régimen dominante en el viento. Recapitulando, este análisis debería ser capaz de responder a los siguientes interrogantes:

(a) ¿Cuáles son los dominios de cada clase de solución en el espacio de los parámetros de la fuerza de radiación?

(b) ¿De qué variables dependen los límites de cada dominio?

(c) ¿Cómo se relaciona la solución $\delta_{\text {lenta }}$ con la rotación estelar?

(d) ¿Cuáles son los valores de $\delta$ que producen un cambio de régimen y cómo se desarrolla esa transición?

(e) ¿Cómo se vincula la solución $\delta_{\text {lenta }}$ con los parámetros fundamentales de la estrella, tales como la temperatura efectiva, la masa y el radio estelar, la metalicidad, etc.?

Finalmente, la pregunta más relevante que se desea responder es: ¿Puede modelarse el viento de una estrella B supergigante por medio de la solución $\delta_{\text {lenta }}$ ?

La mayor parte de los resultados de estos análisis fueron presentados por el autor en el trabajo Venero, Curé, Cidale, \& Araya (2016b) y son desarrollados, con mayor número de detalles e información adicional, en este capítulo.

\section{2. "Modelos base" para las estrellas supergigan- tes B}

Para modelar las estrellas supergigantes B es necesario, en primera instancia, establecer los parámetros fundamentales representativos de las mismas, de acuerdo a los valores convenientes presentados en la sección 1.2.3. En este trabajo, se denomina " $m o$ delo base" a cada conjunto de estos parámetros (tabla 4.1), construido para representar un subtipo espectral dentro de las estrellas supergigantes B.

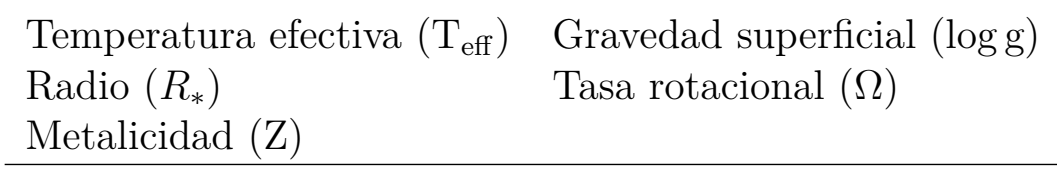

Tabla 4.1. Parámetros que definen a un modelo base.

Los demás parámetros que caracterizan a una estrella, tales como la masa, la luminosidad, el parámetro de Eddington $\Gamma$ o la velocidad de escape, entre otros, pueden calcularse a partir de los parámetros fundamentales que componen un modelo base.

En la tabla 4.2 se muestran los siete modelos base elegidos para analizar en esta tesis, ordenados según sus temperaturas efectivas. Estos modelos base pueden agruparse en tres conjuntos representativos (columna 6) de los subtipos B tardíos (B4 a B9), intermedios (B1.5 a B3) y tempranos (B0 a B1). Los contenidos restantes de la tabla son: el nombre breve del modelo base en la columna 1, la temperatura efectiva $\left(T_{\text {eff }}\right)$ 
y los valores representativos de la gravedad superficial de la estrella (log $g$ ) y el radio estelar $\left(R_{*}\right)$, se tabulan en las columnas 2,3 y 4 . La columna 5 muestra el tipo espectral aproximado que se corresponde con esos parámetros, de acuerdo a la literatura (sección 1.2.3). Los parámetros fundamentales de estos modelos base fueron elegidos tomando como referencia a las citas bibliográficas tabuladas en la columna 7.

En todos los casos, la composición química se ha ajustado a valores similares a los del Sol (para los valores usados, ver la sección 2.2).

\begin{tabular}{cccclll}
\hline \hline Nombre & $\begin{array}{c}T_{\text {eff }} \\
{[K]}\end{array}$ & $\begin{array}{c}\log g \\
{[\mathrm{cgs}]}\end{array}$ & $\begin{array}{c}R_{*} \\
{\left[R_{\odot}\right]}\end{array}$ & $\begin{array}{l}\text { Tipo } \\
\text { Espectral }\end{array}$ & Grupo & Fuente \\
\hline T13 & 13000 & 1.73 & 68 & B8 Iae & Tardías & M08 \\
T15 & 15000 & 2.11 & 52 & B5 Ib & Tardías & S08 \\
T17 & 17000 & 2.24 & 56 & B2.5 Ia & Intermedias & S08 \\
T19 & 19000 & 2.50 & 40 & B2 I & Intermedias & S08 \\
T21 & 21000 & 2.67 & 35 & B1 Ib & Tempranas & S08 \\
T23 & 23000 & 2.72 & 35 & B0.7 Ia & Temparanas & S08 \\
T25 & 25000 & 2.90 & 35 & B0.5 Ia & Tempranas & P86 \\
\hline
\end{tabular}

Tabla 4.2. Parámetros adoptados para la construcción de los modelos base. Las citas de la tabla son: M08: Markova \& Puls (2008), S08: Searle et al. (2008) y P86: Pauldrach et al. (1986)

Cada modelo de viento rotante impulsado por radiación, se construye sobre un dado modelo base, a partir de un subconjunto de parámetros de la fuerza de radiación: $k, \alpha$ y $\delta$.

Es importante notar que aún no se han llevado a cabo, cálculos de los parámetros $\alpha, k$ y $\delta$, en el régimen de la solución lenta, de manera que sean consistentes con la estructura de temperatura y las variables hidrodinámicas del viento. Se trata de cálculos complejos, en los que se está comenzando a progresar. Hasta tanto no se cuente con modelos consistentes, estos parámetros deberán ser ensayados por prueba y error, calibrando adecuadamente su influencia sobre las características observables del espectro.

\subsection{Dominios en el espacio de $\delta$ y $\Omega$}

En esta sección se explora la dependencia de las soluciones y los valores de los parámetros globales del viento $v_{\infty}$ y $\dot{M}$, en relación con dos variables importantes: la rotación, caracterizada por la tasa rotacional $\Omega$ y la ionización del medio, a través del parámetro $\delta$.

Para esto, se construye, sobre cada modelo base de la tabla 4.2, una delgada grilla considerando valores de $\Omega$ comprendidos entre $\Omega=0$, que corresponde a una estrella sin rotación, y $\Omega=0.9$, que representa a un objeto muy cerca del límite de ruptura por las fuerzas centrífugas. La grilla en $\Omega$ tiene intervalos de 0.1 , lo cual permite obtener un muestreado detallado de los cambios producidos sobre el viento por la rotación estelar, entre un modelo y otro. En general, las estrellas B supergigantes son rotadores 
lentos, sin embargo, algunas clases especiales de estrellas pertenecientes a estas clases espectrales poseen altas tasas de rotación, como es el caso de las estrellas B [e] supergigantes (sección 1.2.1). En ese sentido, la grilla contempla toda la amplitud de casos, independientemente de la identificación de un grupo de objetos en particular.

Para abarcar la variedad completa de soluciones generadas por cambios en la ionización, se deben asignar valores a los parámetros $\alpha$ y $k$ de la fuerza de radiación, los cuales serán considerados como constantes en esta parte del análisis. Los valores adoptados para estos parámetros se encuentran en la Tabla 4.3, junto con las referencias de las fuentes usadas para la elección de dichos valores.

\begin{tabular}{cccc}
\hline \hline Nombre & $k$ & $\alpha$ & Fuente \\
\hline T13 & 0.30 & 0.50 & A82 \\
T15 & 0.32 & 0.50 & A82 \\
T17 & 0.34 & 0.50 & A82 \\
T19 & 0.32 & 0.50 & P00-P86 \\
T21 & 0.32 & 0.50 & A82-P86 \\
T23 & 0.34 & 0.53 & A82 \\
T25 & 0.34 & 0.55 & P86 \\
\hline
\end{tabular}

Tabla 4.3. Parámetros de la fuerza de radiación adoptados en las grillas de modelos. Las citas de la tabla son: A82: Abbott (1982b), P86: Pauldrach et al. (1986) y P00: Pelupessy et al. (2000)

Para evaluar la variación de la ionización, se introduce un rango de valores para el parámetro $\delta$ que se extiende entre $\delta=0$, que corresponde a una solución en la cual el estado de ionización se mantiene constante a lo largo de todo el viento (frozen in ionization), hasta $\delta=0.4$, que indica un profundo cambio en las condiciones de ionización al aumentar la distancia a la estrella (sección 2.2.6). Los intervalos considerados en esta grilla de $\delta$ son de una amplitud de 0.01 , lo cual genera una grilla muy detallada que se requiere para determinar los límites de las regiones comprendidas entre la solución rápida y la $\delta_{\text {lenta }}$.

Para cada modelo base de la tabla 4.2, combinado con los parámetros adoptados para $k$ y $\alpha$, y los correspondientes valores de la grilla de $\delta$, se calcula una solución de las ecuaciones hidrodinámicas usando el código HYDWIND (sección 3.3.5). Cada solución para el viento se caracteriza por su valor de $v_{\infty}$ y $\dot{M}$.

\subsubsection{Soluciones para el modelo base T19}

Los valores de $v_{\infty}$ y $\dot{M}$ obtenidos de la manera mencionada para el modelo base T19, se encuentran en la tabla 4.4. En este trabajo, el modelo base T19 es adoptado como modelo representativo para mostrar el comportamiento de las soluciones en las supergigantes B intermedias y tardías, cuyo valor de $T_{\text {eff }} \leq 23000 \mathrm{~K}$. Para temperaturas efectivas mayores, el comportamiento de las soluciones se describe en la sección 4.3.3.

Para algunas combinaciones de los parámetros $\Omega$ y $\delta$, no se encuentran soluciones estacionarias con el código HYDWIND, razón por la cual se dejan los espacios vacíos en la Tabla 4.4. Como se menciona en la sección 3.3.5, el código HYDWIND requiere 


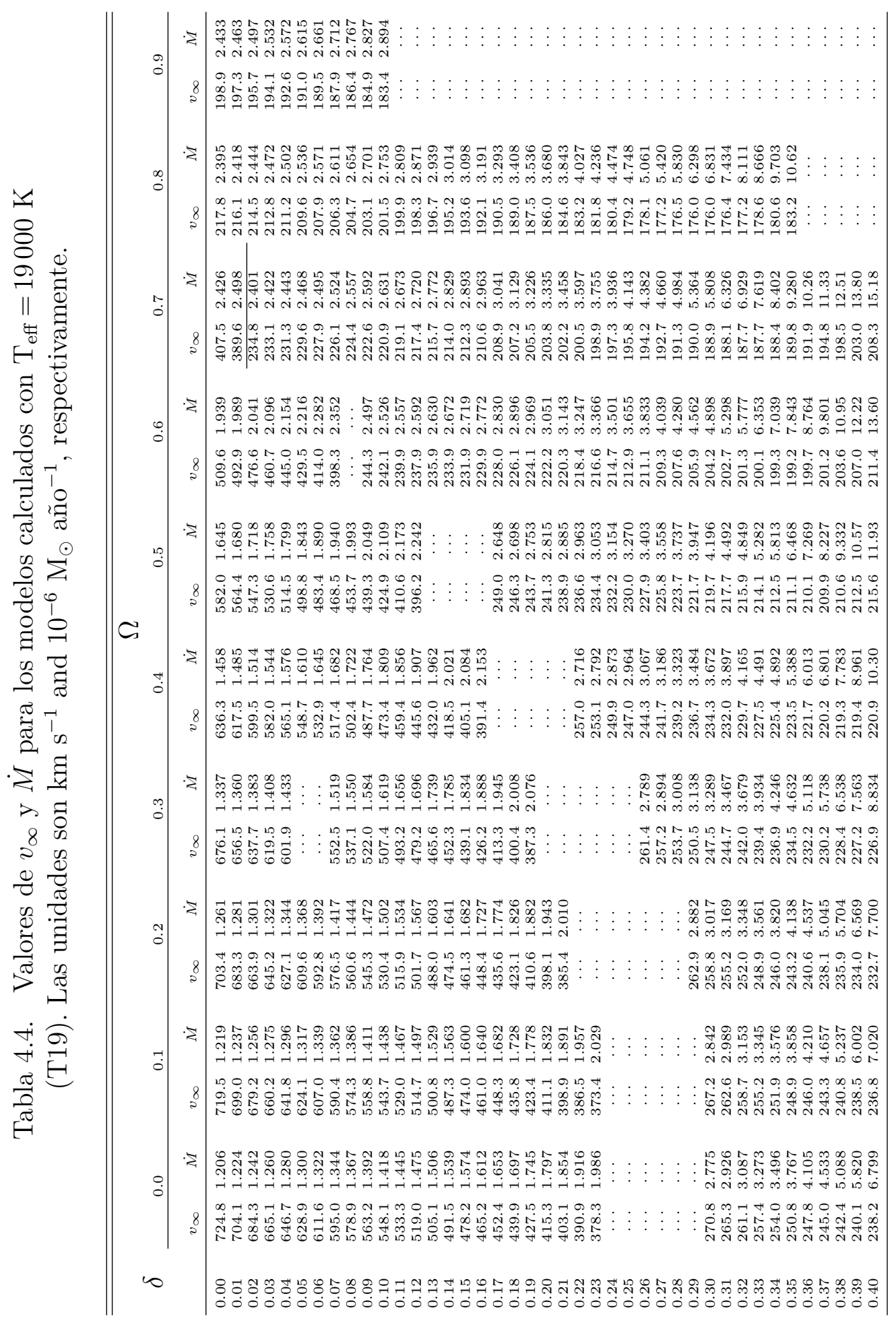


de una ley de velocidades de prueba inicial, la que acelera la convergencia de las ecuaciones. Esto requiere que los parámetros de dicha función de prueba sean asignados secuencialmente por prueba y error, buscando una solución correctamente convergida. Antes de dejar vacío un casillero de la Tabla 4.4. se verifican previamente alrededor de 6000 casos con parámetros de prueba en todo el rango de valores posibles. Estas pruebas aseguran que, para esas combinaciones específicas de $\Omega$ y $\delta$, no hay una solución estacionaria.

Todos los valores de los modelos de la tabla 4.4 fueron obtenidos usando la condición inicial de $\tau=2 / 3$ en $\mathrm{r}=\mathrm{R}_{*}$.

\section{Relaciones de escala para el modelo base T19}

Un primer examen de las soluciones hidrodinámicas consiste en comparar la dependencia de los valores obtenidos para $\mathrm{v}_{\infty}$ y $\dot{M}$ en función de la tasa rotacional $\Omega$, con las formas previstas por las relaciones de escala (sección 3.2.1) que pueden verse en la figura 3.3, publicada por Puls et al. (1999). Los resultados para los modelos calculados en este trabajo se muestran en la figura 4.1, en el cual se incorporan las curvas correspondientes a diferentes valores del parámetro $\delta$.

En esta figura puede verse que la dependencia de los parámetros del viento con $\Omega$ presentan un comportamiento acorde al presentado por Puls et al. (1999). La principal diferencia está en los valores de $\mathrm{v}_{\infty}$ y de $\dot{M}$, ya que los cálculos de ese trabajo se han hecho para una supergigante $\mathrm{O}$, a partir de la adopción de una ley $\beta$ de velocidades. En cambio, la figura 4.1 corresponde a los valores para una supergigante B (modelo base T19), para la cual la velocidad terminal y la tasa de pérdida de masa son considerablemente menores.

Puede notarse además que, en el gráfico de Puls et al. (1999), los valores de $\Omega$ sólo alcanzan el valor 0.6, por lo cual se excluyen los modelos con alta rotación. Modelos con valores grandes de $\Omega(\Omega>0.6)$ pueden calcularse con el código HYDWIND, dando lugar a la solución $\Omega_{\text {lenta }}$. Por esa razón, en la figura 4.1 aparece un cambio brusco en la pendiente de las curvas para valores altos de $\Omega$, tanto de $\mathrm{v}_{\infty}$ como de $\dot{M}$. También, en esta figura, puede observarse la aparición de la solución $\delta_{\text {lenta }}$, la cual es dominante para valores de $\delta$ mayores que 0.25 (curva en color amarillo y sucesivas).

\section{Distribución de soluciones para el modelo base T19}

Para identificar las regiones del espacio de los parámetros $\Omega$ y $\delta$, dominadas por cada una de las clases de soluciones hidrodinámicas, es conveniente realizar un gráfico cuya abcisa sea el parámetro $\delta$. La figura 4.2 muestra el comportamiento de las soluciones para el modelo base T19, con las velocidades terminales (panel izquierdo) y las tasas de pérdida de masa, graficados en función del parámetro $\delta$. Cada símbolo representa uno de los modelos calculados y cada línea de color une los modelos con valores comunes de la tasa rotacional $\Omega$.

A partir de la tabla 4.4 y de la figura 4.2 , puede hacerse una interpretación directa de la distribución de soluciones, en vista de los valores obtenidos para $v_{\infty}$ y $\dot{M}$ como función de $\delta$. Los dominios se establecen siguiendo algunos patrones:

- Solución rápida: Las soluciones obtenidas para valores bajos de $\delta$ corresponden a vientos descritos por la solución rápida, siempre que la tasa rotacional no 

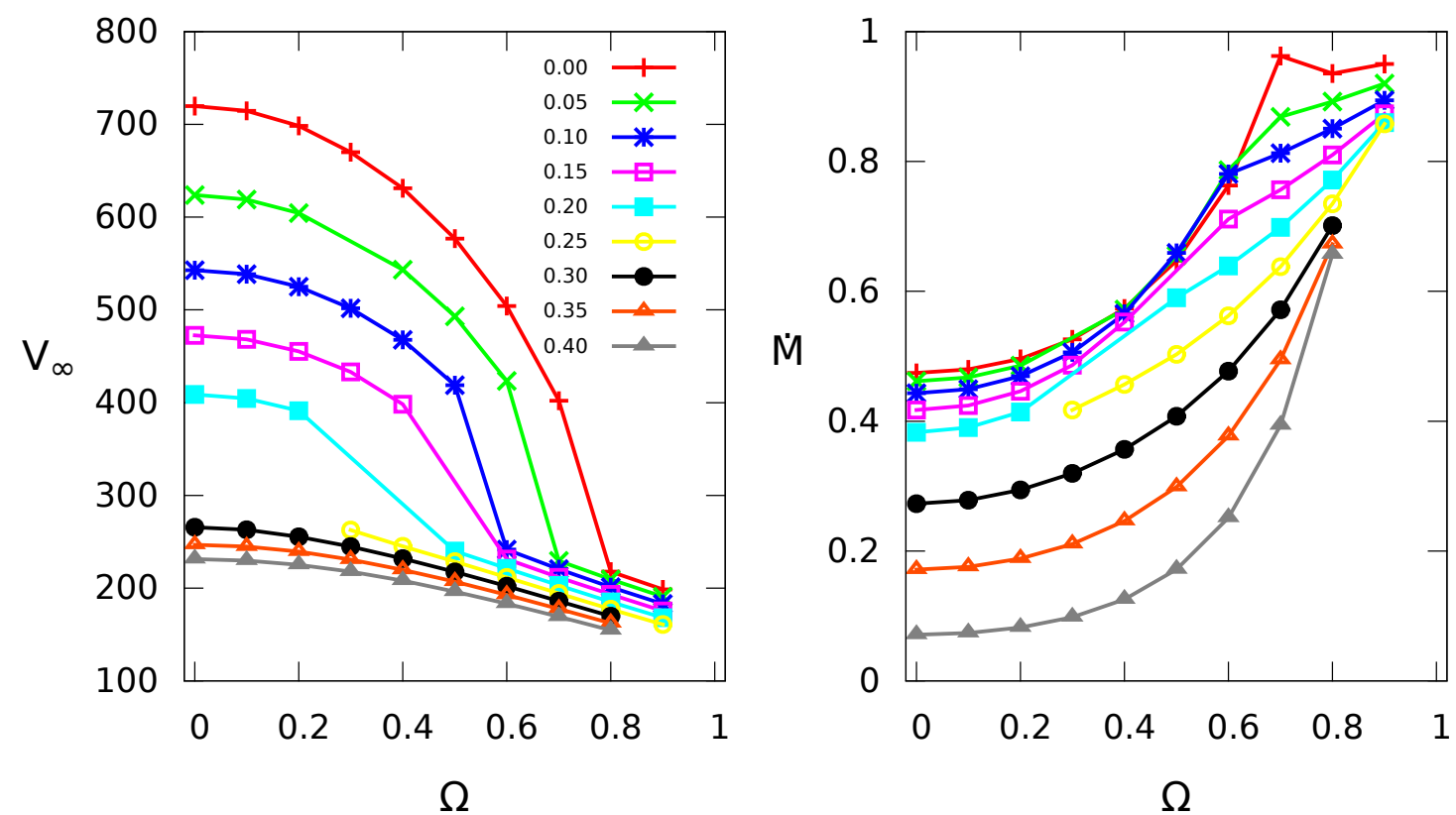

Figura 4.1. Valores de $\mathrm{v}_{\infty}\left[\mathrm{km} \mathrm{s}^{-1}\right]$ y $\dot{M}\left[10^{-6} \mathrm{M}_{\odot}\right.$ año $\left.{ }^{-1}\right]$ en función de la tasa rotacional $\Omega$. Cada punto muestra un modelo calculado, mientras que cada curva corresponde a un modelo con un valor fijo del parámetro de ionización $\delta$, que se representa con curvas de diferentes colores cuyos valores se encuentran en el gráfico de la izquierda.

exceda el valor aproximado de 0.8. A este dominio le corresponden las curvas descendentes de la izquierda del panel correspondiente a $\mathrm{v}_{\infty}$ vs $\delta$ en la figura 4.2. Estas curvas coinciden con las ramas inferiores que se encuentran hacia la izquierda del panel que corresponde a $\dot{M}$ vs $\delta$.

- Solución $\Omega_{\text {lenta }}$ : Los modelos que surgen para $\Omega \gtrsim 0.8$ (curvas gris y roja) corresponden al régimen $\Omega_{\text {lento }}$. No puede distinguirse una separación entre estos modelos y los que corresponden a la solución $\delta_{\text {lenta }}$, exceptuando por la alta rotación que caracteriza a los primeros.

- Brecha: Para modelos con $\delta$ mayores que un valor $\delta_{\mathrm{b} 1}$ dado, que depende fuertemente de $\Omega$, la solución rápida desaparece. Para estos valores de $\delta$ ya no pueden encontrarse soluciones estacionarias. Esta ausencia de soluciones continúa en un intervalo de valores de $\delta$ hasta un valor máximo $\delta_{\mathrm{b} 2}$. A esa región en el espacio de parámetros $(\Omega, \delta)$ se la llamará brecha (gap), y en su extensión no habrá soluciones estacionarias.

- Solución $\delta_{\text {lenta }}$ : Para valores de $\delta \geq \delta_{\mathrm{b} 2}$, reaparecen las soluciones estacionarias, pero ahora son soluciones del tipo $\delta_{\text {lenta }}$, con velocidades terminales sensiblemente menores y mayores tasas de pérdida de masa que en los casos con solución rápida.

Cada uno de estos dominios se encuentra, aproximadamente, indicado mediante rótulos en la figura 4.4 . 

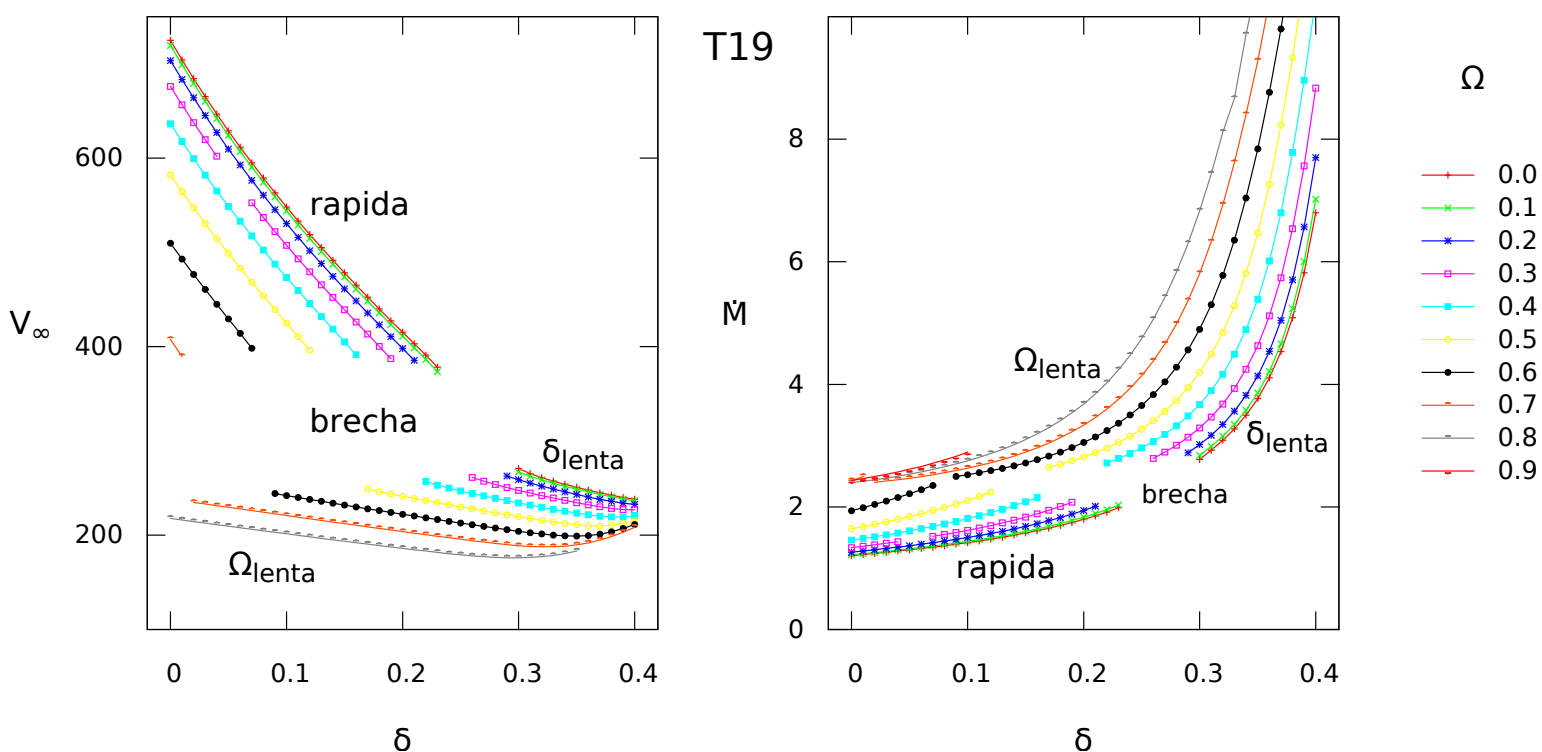

Figura 4.2. Izquierda: Velocidades terminales $\left[\mathrm{km} \mathrm{s}^{-1}\right]$ como función del parámetro $\delta \mathrm{y}$ la tasa de rotación (en líneas de color con puntos) para el modelo base T19. Derecha: Tasas de pérdida de masa $\left[10^{-6} \mathrm{M}_{\odot}\right.$ año $\left.{ }^{-1}\right]$ como función de $\delta$ y $\Omega$ (indicadas en la referencia). Se puede notar la brecha que separa las soluciones rápidas de las soluciones lentas.
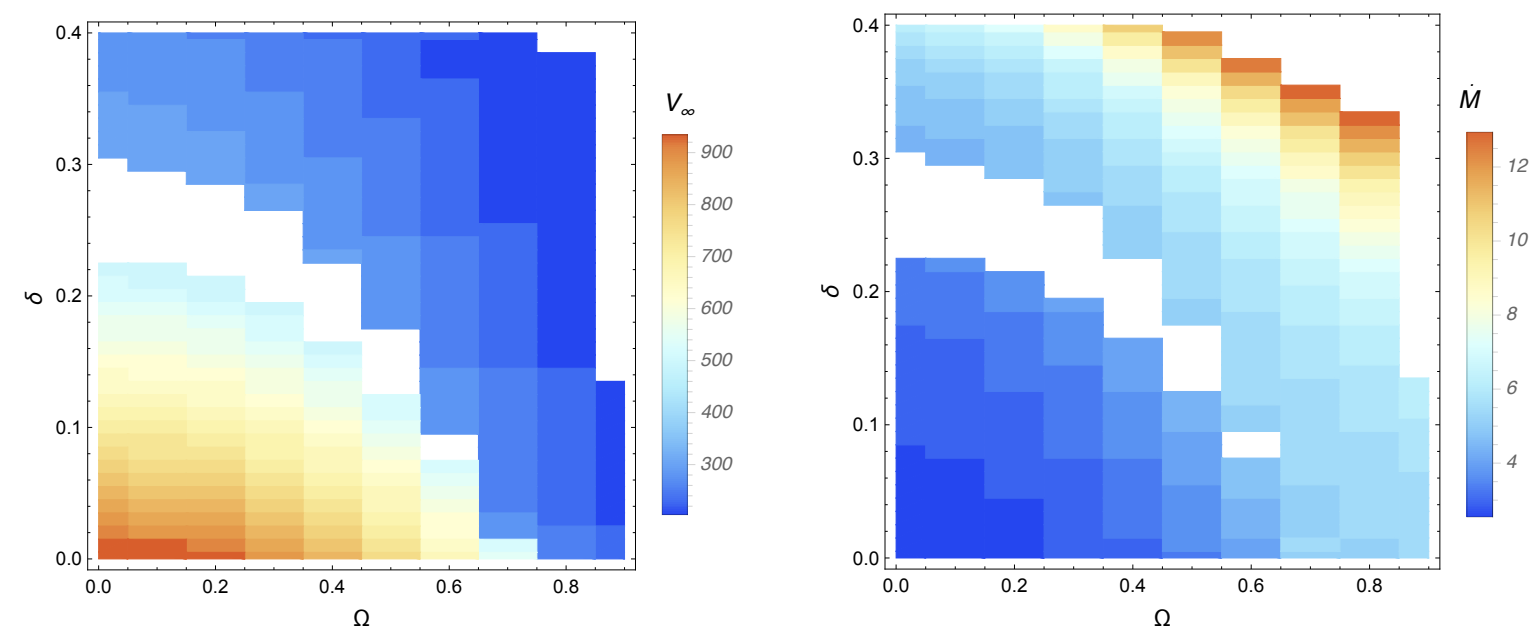

Figura 4.3. Mapas de color que representan el comportamiento de las velocidades terminales en $\mathrm{km} \mathrm{s}^{-1}$ (panel izquierdo) y las tasas de pérdida de masa en unidades de $10^{-6} \mathrm{M}_{\odot}$ año ${ }^{-1}$ (panel derecho) correspondientes al modelo base T19, como función del parámetro $\delta$. En el primer gráfico, la región que queda por debajo de la brecha (región blanca) corresponde a la solución rápida, mientras que la zona azulada de arriba contiene modelos de solución lenta (nótese que la ordenada $\delta$ es creciente hacia arriba en ambas figuras). En el segundo gráfico, la región de debajo de la brecha corresponde al régimen rápido mientras que la región superior, al régimen lento. 


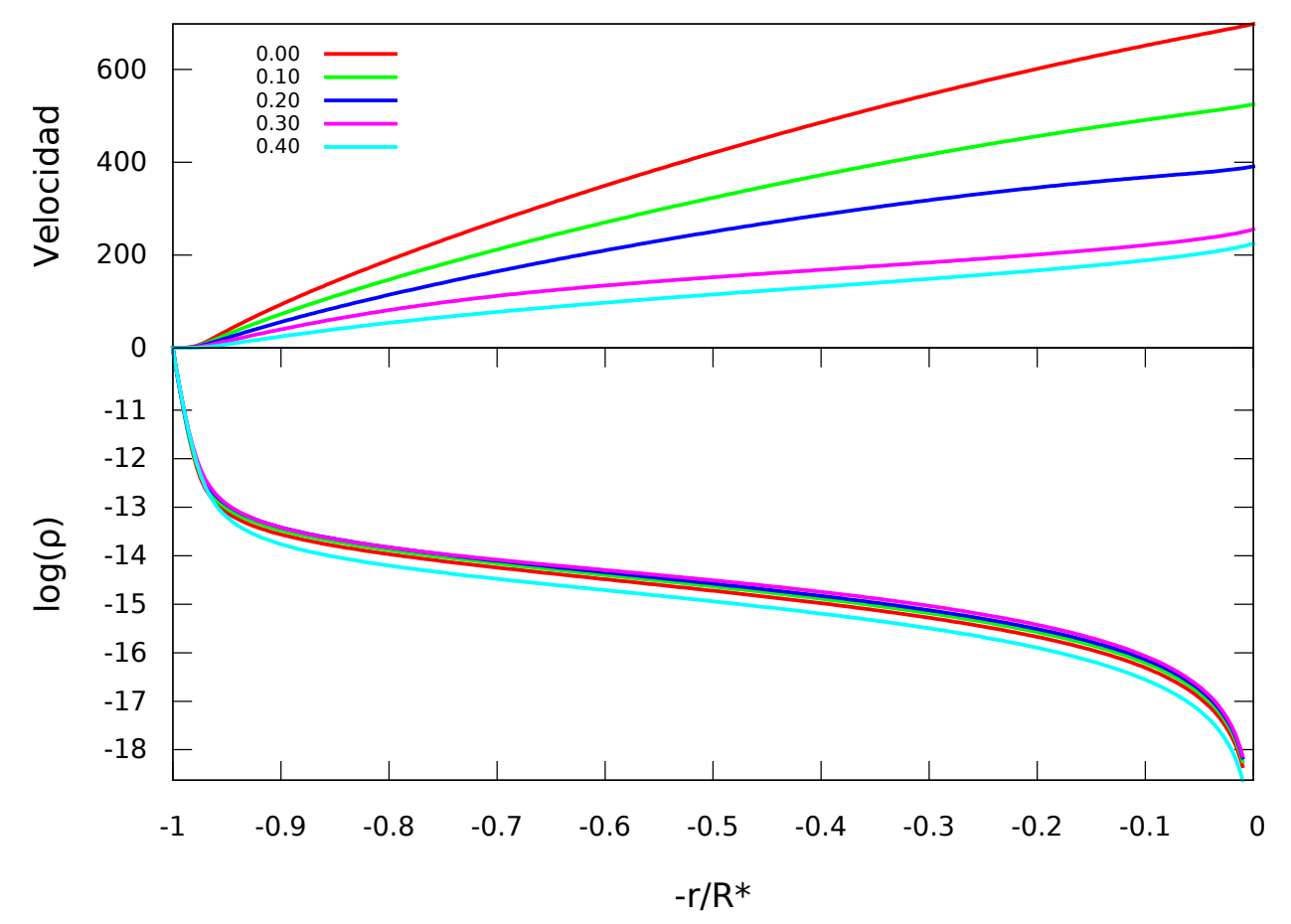

Figura 4.4. Arriba: Velocidad $\left[\mathrm{km} \mathrm{s}^{-1}\right]$ en función de la coordenada $u=-R_{*} / r$. Abajo: Logaritmo de la densidad en función de la misma coordenada radial. Las leyes corresponden al modelo base T19 con $\Omega=0.2, k=0.20$ y $\alpha=0.5$. Se muestran cinco casos correspondientes a diferentes valores del parámetro $\delta$ (citados en la referencia), que van desde $\delta=0$ hasta 0.30 , en el dominio de la solución rápida, y $\delta=0.40$ en el régimen lento. Para $\delta=0.30$, la solución se encuentra muy próxima a la brecha, por lo cual la forma funcional presenta cambios suaves en el gradiente de velocidades.

Las propiedades matemáticas de las soluciones estacionarias para vientos impulsados por radiación en rotación se resumen en la tabla 4.5. El régimen rápido se corresponde con soluciones de vientos con gran aceleración, con cocientes $v_{\infty} / v_{\text {esc }}$ mayores que 0.9, Para el régimen lento los valores son más bajos, como puede verse en la sexta columna de la tabla.

Las variaciones de las velocidades y de la densidad para las diferentes clases de soluciones pueden verse en la figura 4.4. Las diferentes curvas corresponden a varios valores del parámetro $\delta$, mostrando las modificaciones que se producen en la forma en que se distribuyen radialmente estas variables, al modificarse la ionización. Puede notarse la gran diferencia en velocidad terminal entre una solución rápida $(\delta=0)$ y una solución lenta $\delta=0.4$.

\section{El dominio conjunto de solución lenta}

En el dominio de la solución lenta, dos regiones deberían distinguirse, dependiendo de la dominancia de $\delta$ o de $\Omega$ : el régimen $\delta_{\text {lento }}$, caracterizado por altos gradientes de ionización (altos valores de $\delta$ ) y bajos valores de $\Omega$, y el régimen $\Omega_{\text {lento }}$, para rotadores rápidos $(\Omega \geq 0.8)$ y bajos valores de $\delta$. Sin embargo, un rápido examen de la tabla 4.4 
Tabla 4.5. Localización de los dominios y propiedades de las soluciones estacionarias según parámetros de los modelos. Los valores tabulados son una referencia aproximada, tomada del modelo base T19.

\begin{tabular}{cccccc}
\hline \hline Régimen & solución & propiedades & rango de $\Omega$ & rango de $\delta$ & $V_{\infty} / V_{\text {esc }}$ \\
\hline rápido & m-CAK & $\begin{array}{c}\text { gran aceleración } \\
\text { baja rotación } \\
\text { ionización baja }\end{array}$ & $<0.8$ & $\lesssim 0.25$ & $\sim 0.9-2.3$ \\
\hline lento & $\begin{array}{c}\text { m-slow } \\
\text { baja aceleración } \\
\text { alta rotación }\end{array}$ & $\geq 0.8$ & $\lesssim 0.25$ & $\sim 0.5-0.7$ \\
& $\begin{array}{c}\text { baja aceleración } \\
\text { altos grad. ionización }\end{array}$ & $<0.8$ & $>\delta_{\mathrm{b} 2}$ & $\lesssim 0.9$ \\
\hline
\end{tabular}

muestra que no hay ninguna separación o borde nítido entre ambas regiones.

Los parámetros del viento tales como la velocidad terminal o la tasa de pérdida de masa no cambian de manera discontinua ni muestran una brecha como la que separa la solución rápida de la solución lenta. Por esta razón, Venero et al. (2016b) propusieron que toda la región dominada por el régimen lento, sea denominado régimen $\Omega \delta_{\text {lento }}$.

Esta deducción está de acuerdo con las propiedades compartidas por las soluciones rápida y lenta: baja $\mathrm{v}_{\infty}$ y alta densidad del medio. Por simplicidad, en los análisis siguientes se denominará a todo el dominio de la solución $\Omega \delta_{\text {lenta }}$, como la solución lenta en general.

\section{Sobre la brecha del modelo base T19}

Tanto la ubicación como el ancho de la brecha dependen de $\Omega$. Si $\Omega \lesssim 0.8$, la brecha tiene lugar a mayores valores de $\delta$. A medida que la tasa de rotación crece, el valor inicial de la brecha, $\delta_{b 1}$, se reduce y la brecha se vuelve más angosta. Esta disminución de la brecha continúa hasta que la misma desaparece completamente alrededor de $\Omega \approx 0.8$. Para mayores valores de $\Omega$ no existe solución rápida posible, para ningún valor de $\delta$.

Para ejemplificar esta descripción, puede observarse en la tabla 4.4 que, para el modelo T19 no rotante $(\Omega=0)$, el ancho de la brecha $\left(\Delta \delta=\delta_{\mathrm{b} 2}-\delta_{\mathrm{b} 1}\right.$ en escala de valores de $\delta$, es 0.07 , mientras que, para $\Omega=0.6$, la brecha ha disminuido su ancho a 0.02. Para $\Omega=0.7$, la brecha es aún más pequeña (0.01) y está ubicada entre los valores $\delta=0.1$ y $\delta=0.2$. Finalmente para $\Omega \gtrsim 0.8, \Delta \delta=0$. Este comportamiento relativo a la ubicación y el angostamiento en la brecha puede verse claramente en la figura 4.2 .

Como se mencionó antes, para $\delta \geq \delta_{\mathrm{b} 2}$, es decir, más allá de la brecha, se encuentra el dominio de la solución $\delta_{\text {lenta }}$ donde la variación de la velocidad terminal es pequeña en contraste con el comportamiento de $\dot{M}$, que crece fuertemente a medida que $\delta$ es 
incrementado. Estos cambios en los parámetros del viento pueden observarse en la Fig 4.2 .

Adicionalmente, la figura 4.3 muestra una representación en mapas de color para los diferentes dominios de las soluciones para el modelo base T19. En estos diagramas, puede distinguirse la brecha entre las soluciones rápida y lenta (región en blanco). También puede observarse que no hay un límite definido entre las soluciones $\delta_{\text {lenta }} \mathrm{y}$ $\Omega_{\text {lenta. }}$.

La velocidad de rotación contribuye a aumentar la extensión del dominio de la solución $\delta_{\text {lenta. }}$ A medida que la tasa de rotación crece, la brecha se desplaza hacia valores más bajos de $\delta$ y el dominio de la solución $\delta_{\text {lenta }}$ avanza sobre el dominio de la solución rápida.

Un análisis más detallado acerca de la brecha y sus propiedades para todos los modelos base en general, es desarrollado en la sección 4.6 .

\subsubsection{Soluciones para modelos base de temperaturas efectivas bajas e intermedias}
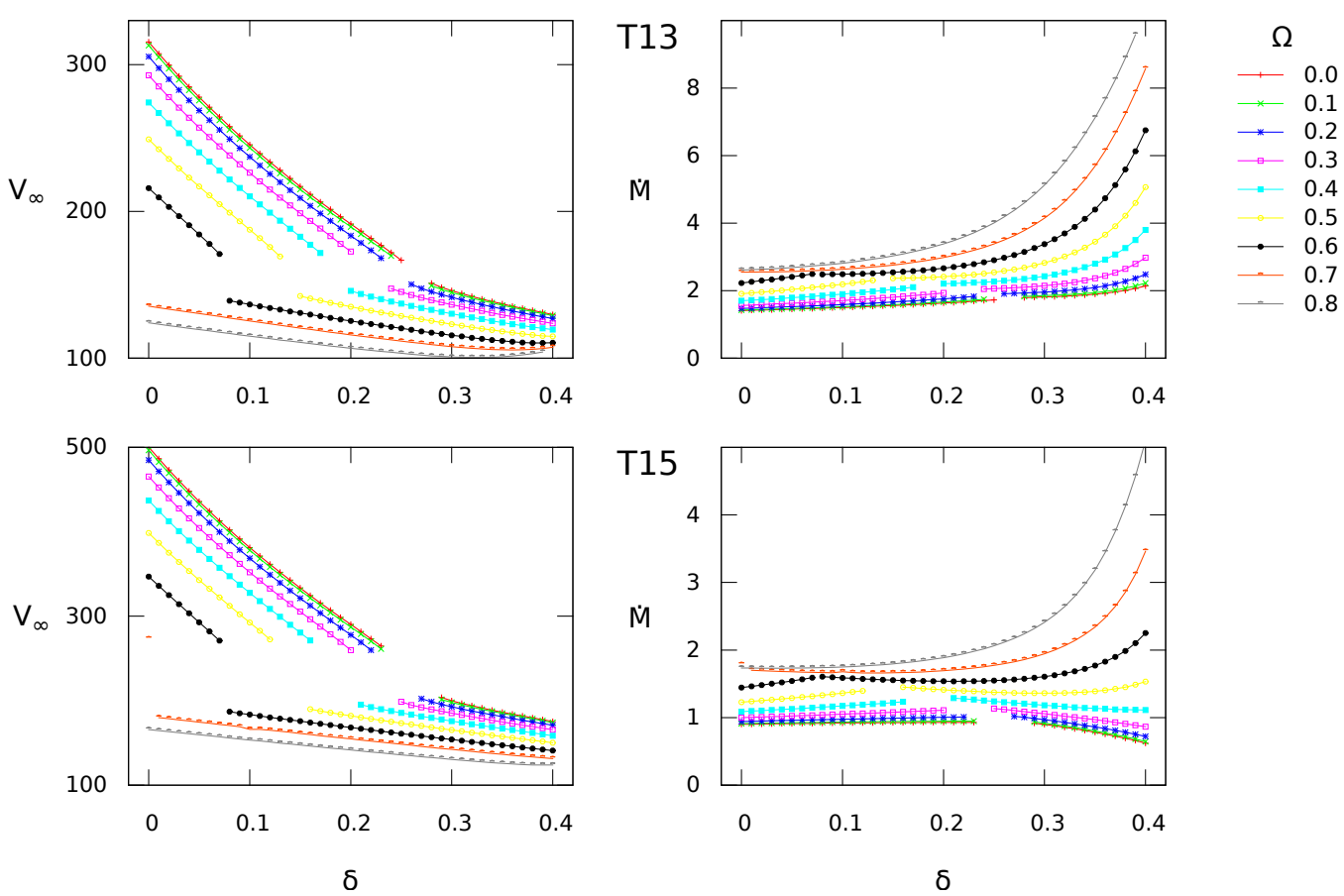

Figura 4.5. Velocidades terminales (paneles izquierdo) y tasas de pérdida de masa (paneles derecho) en función del parámetro $\delta$ con diferentes valores de $\Omega$ para los modelos base T13 y T15. Estos gráficos muestran una distribución de dominios análoga a la descripta en la sección 4.3.1, con una brecha dividiendo claramente los regímenes rápido y lentos.

Todos los modelos base con $\mathrm{T}_{\text {eff }} \leq 21000 \mathrm{~K}$ muestran un comportamiento análogo al descripto en la sección anterior para T19, como puede verse en las figuras 4.5 y 4.6 . 

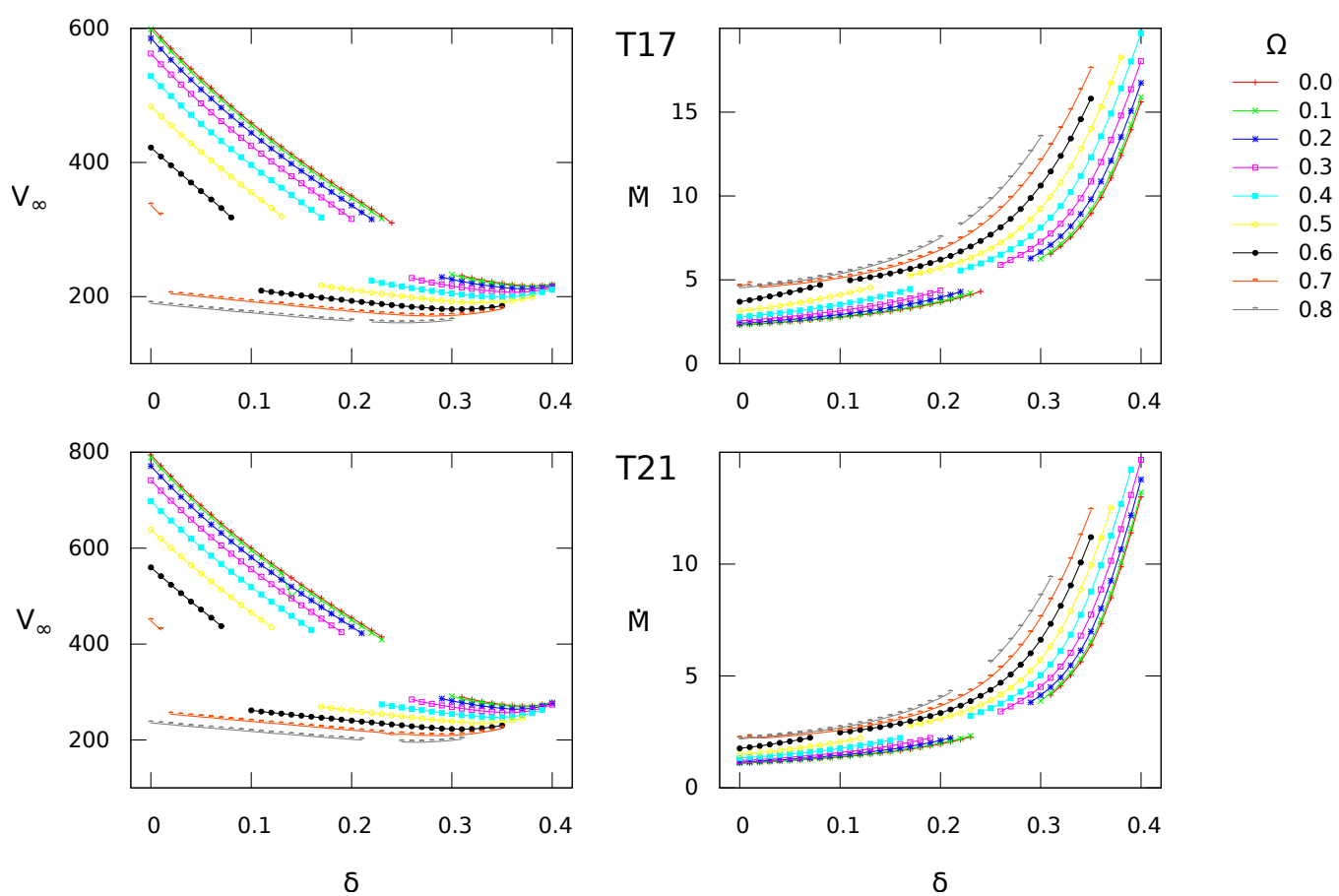

Figura 4.6. Lo mismo que en la figura 4.5 pero para los modelos base T17 y T21.

Esto significa que los modelos corresponden a la solución rápida si los valores de $\delta$ son menores que $\delta_{\mathrm{b} 1}$, y a una solución lenta si los valores de $\delta$ son mayores que $\delta_{\mathrm{b} 2}$. Ambas clases de soluciones están separadas por una brecha. También, para rotadores rápidos $(\Omega \geq 0.8)$ no hay solución rápida para cualquier valor de $\delta$, en acuerdo con lo demostrado por Curé (2004). Nótese, no obstante, las diferencias en los valores de las velocidades terminales y tasas de pérdida de masa alcanzadas en comparación entre un modelo base y otro.

\subsubsection{Soluciones para modelos base de alta temperatura efec- tiva}

Resulta interesante analizar separadamente el comportamiento de los modelos base con temperaturas efectivas iguales o mayores que $23000 \mathrm{~K}$, ya que los mismos parecen mostrar un comportamiento diferente al presentado en la sección anterior. En el trabajo original de Venero et al. (2016b), se reportó que no era posible alcanzar la convergencia para modelos en ese rango de temperaturas efectivas para ningún valor de $\delta>\delta_{\mathrm{b} 1}$, dada cualquier tasa rotacional $\Omega$. Esto implica que los modelos base calculados sólo convergen en el caso de régimen rápido, es decir para valores de $\delta$ menores que los valores típicos comprendidos entre 0 y 0.25 . Las curvas correspondientes a $\mathrm{v}_{\infty}$ y $\dot{M}$ para diferentes valores de $\delta$ y $\Omega$ para estos casos, pueden verse en la figura 4.7 .

Sin embargo, esta imposibilidad de obtener soluciones lentas para las estrellas supergigantes B más tempranas está en contradicción con lo reportado por Curé $(2004)$ y Madura et al. (2007), quienes encontraron esta clase de régimen de viento para modelos de altos rotadores con temperaturas efectivas mayores o iguales a $25000 \mathrm{~K}$. La 

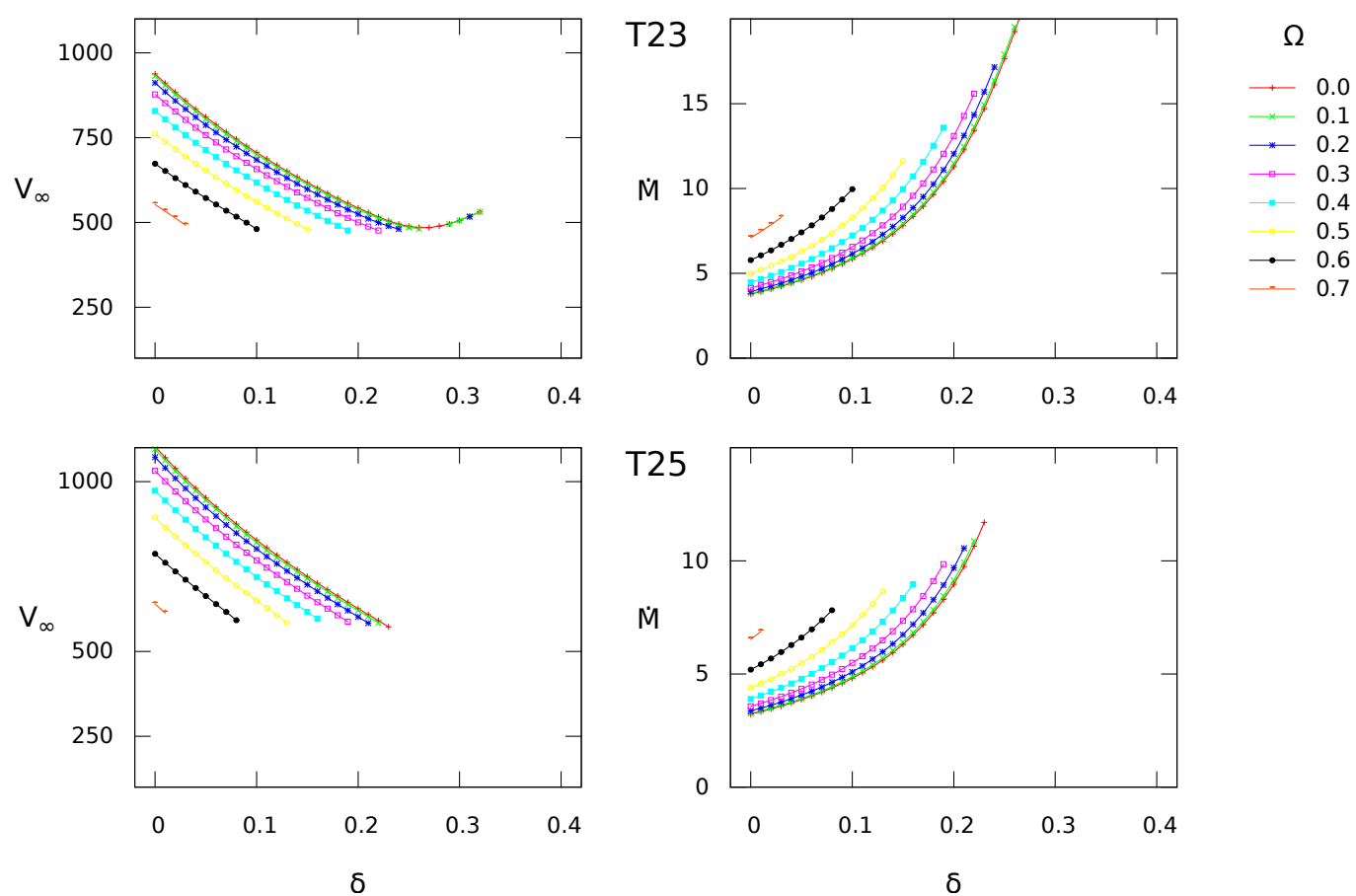

Figura 4.7. Lo mismo que en la figura 4.5 pero para los modelos base T23 y T25. Nótese la ausencia de solución lenta. El valor adoptado para el parámetro $k$ en estos modelos es 0.34 , como lo indica la Tabla 4.3 .

justificación de la ausencia de la solución lenta en este subgrupo de modelos se discute en la sección 4.4.1.

\subsection{Influencia del parámetro $k$ en los dominios de las soluciones}

Tal como se vio en la sección 2.2.5, el parámetro $k$ está relacionado estrechamente al número efectivo de líneas que impulsan al viento. Por este motivo, su valor es proporcional a la cantidad de gas fluyendo en el viento, es decir, a la tasa de pérdida de masa, y a la metalicidad. La influencia del parámetro $k$ sobre el viento se manifiesta apenas éste comienza a desarrollarse, en proximidades de la fotosfera, donde la densidad alcanza su máximo valor. Por lo tanto, a mayores valores de $k$, mayores tasas de pérdida de masa.

Esta dependencia puede verificarse en la figura 4.8, que muestra las formas funcionales de $\mathrm{v}_{\infty}$ (izquierda) y $\dot{M}$ (derecha) frente a variaciones de $\delta$ pero, en este caso, para un único valor fijo de $\Omega$ (por simplicidad se adoptan modelos no rotantes, $\Omega=0$ ). En esta figura, cada curva es obtenida usando diferentes valores del parámetro $k$, los que se indican en la referencia de la derecha del gráfico. Para la solución lenta, la tasa de pérdida de masa es mucho más sensible al aumento de $\delta$ que para la solución rápida, como puede verse en el panel derecho de la figura 4.8 .

La adición de rotación a estos modelos contribuye a incrementar aún más los valores 


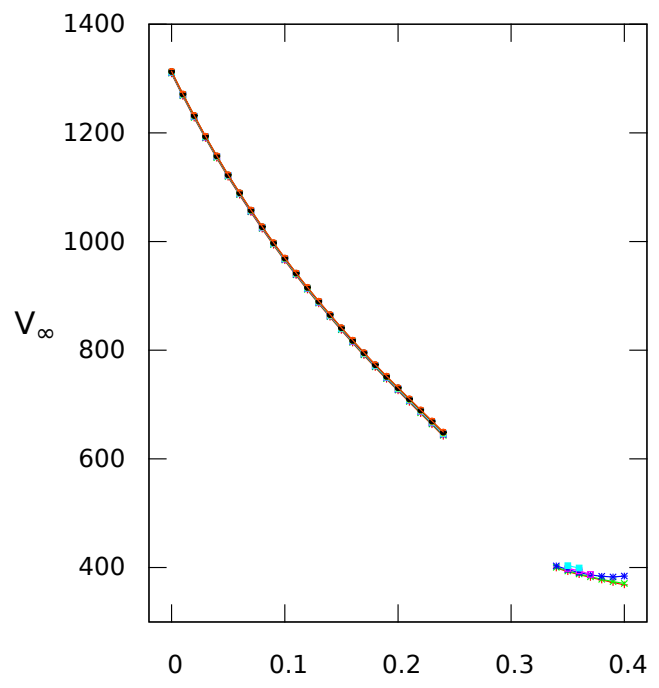

$\delta$

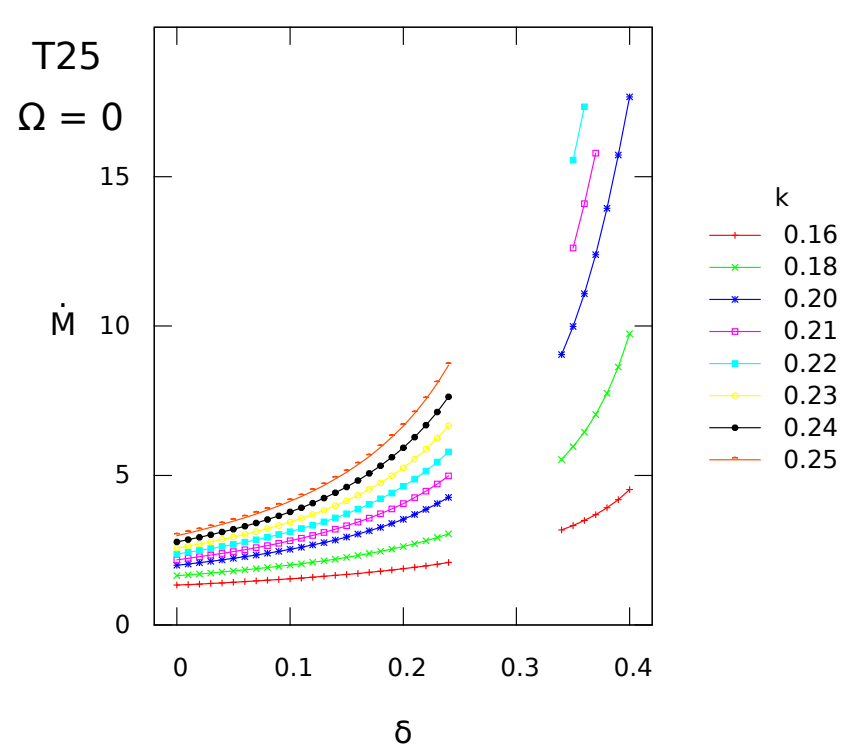

Figura 4.8. Izquierda: Velocidades terminales $\left[\mathrm{km} \mathrm{s}^{-1}\right]$ en función del parámetro $\delta$ para diferentes valores del parámetro $k$, calculados para el modelo base T19 con rotación nula $(\Omega=0)$ y valor fijo de $\alpha=0.5$. Los valores de $\mathrm{v}_{\infty}$ son totalmente insensibles a los cambios en el parámetro $k$. Derecha: Tasas de pérdida de masa $\left[10^{-6} M_{\odot}\right.$ año $\left.{ }^{-1}\right]$ como función de $\delta$ para los mismos modelos. Puede notarse que la brecha que separa las soluciones rápidas y las lentas, no cambia de posición ni de ancho ante variaciones de $k$. Para valores altos de $k$, las tasas de pérdida de masa se vuelven desmesuradamente grandes.

de $\dot{M}$, especialmente en el régimen lento. Con esta condición se le podría poner una cota a los valores de $k$ que se consideren, para evitar vientos con tasas de pérdida de masa excesivas para las supergigantes B.

Llamativamente, la velocidad terminal se mantiene sin cambios en su dependencia con $\delta$, para cualquier valor de $k$, como puede verse en el panel izquierdo de la figura 4.10. Ese gráfico muestra entonces que la velocidad terminal $v_{\infty}$ no depende de $k$, ya que todas las curvas de $v_{\infty}$ se solapan, aún para los valores más grandes de $k$ considerados.

La razón es que $v_{\infty}$ se alcanza muy lejos de la superficie estelar, a una distancia a la cual la mayor parte del momento del campo de radiación ya ha sido completamente transferido al fluido. De esta manera, $k$, que depende de la cantidad de líneas efectivas disponibles para absorber momento, no tiene efectos sobre $v_{\infty}$. En cambio, el valor de $v_{\infty}$ depende del grado de acoplamiento entre los iones y la totalidad del gas. Por esta razón, $v_{\infty}$ siempre es una función decreciente de $\delta$. El incremento de la ionización a lo largo del viento reduce la capacidad de los iones de transferir momento a los elementos mayoritarios, y siempre actúa de la misma manera (Abbott 1982b). Esto se debe a que los iones de alto nivel de ionización tienen menos líneas capaces de absorber momento del campo de radiación. 


\subsubsection{La solución lenta en modelos base de alta temperatura efectiva}

La causa de la ausencia de la solución lenta en el rango de las temperaturas efectivas mayores, mencionada en la sección 4.3.3, es consecuencia de la elección de valores considerablemente altos para el parámetro $k$. Estos valores inadecuados para $k$ son mayores o iguales a 0.3 , como pueden verse en la tabla 4.3 .

La figura 4.8 pone en evidencia que el régimen lento también puede existir para las estrellas más calientes consideradas pero, con la condición de que, en su viento, el parámetro $k$ se mantenga acotado a valores convenientemente bajos.

En el caso anterior para el cual no se encontraba solución lenta (figura 4.7), el valor adoptado para $k$ era 0.34. A partir del panel derecho de la figura 4.8 puede verse que los valores de $\dot{M}$ para $\delta$ grandes, en el modelo base T25 con $k=0.34$, serían excesivamente grandes. Esos valores excesivos de $\dot{M}$ impiden la convergencia correcta del código HYDWIND, razón por la cual, no se obtienen soluciones lentas. Pero, aún en el caso en que pudieran encontrarse estas soluciones, las mismas representarían vientos demasiado intensos, completamente inapropiados para modelar los vientos observados en las supergigantes B.

Un factor que puede influir en la falta de convergencia del código HYDWIND es la elección de la condición inicial $\tau=2 / 3$ para $r=R_{*}$ (ecuación 3.18). La condición dada por la fórmula 3.19 puede evitar esta falta de convergencia (Curé, comunicación privada). Sin embargo, resulta importante que todos los modelos calculados en este trabajo compartan la misma condición inicial, para no introducir factores adicionales que puedan sesgar los resultados.

Esta circunstancia permite poner una cota al valor del parámetro $k$ para la factibilidad de vientos con régimen lento en las supergigantes B más tempranas. A partir del panel derecho de la figura 4.8 puede estimarse, como límite máximo, un valor de $k \sim 0.20$ para la solución rápida (curvas de la izquierda) y uno de $k \sim 0.16$ para la solución lenta, ya que mayores valores de $k$ generarían tasas de pérdida de masa extremadamente altas, no observadas en esta clase de estrellas.

En resumen, en la medida en que el balance de ionizaciones genere valores grandes para el parámetro $\delta$, el parámetro $k$ debe mantenerse en valores bajos o intermedios.

Con valores más apropiados de $k$, en particular $k$ más bajos, puede redefinirse la figura 4.7, dando lugar a la figura 4.9, la cual muestra un comportamiento similar al de los casos de temperaturas efectivas bajas e intermedias.

\subsubsection{La brecha y el parámetro $k$}

La figura 4.8 muestra que la brecha que separa los regímenes rápido y lento es completamente insensible a los cambios en el parámetro $k$. En cambio, tanto el ancho como la ubicación de la brecha dependen, efectivamente, de los parámetros $\delta$ y $\Omega$ y, como se ve en la sección 4.5.1, también de $\alpha$.

Para el modelo base T19, bajo todos los valores de $k$ probados, la brecha se mantiene fija en un rango de $\delta$ comprendido entre 0.22 y 0.30 . 

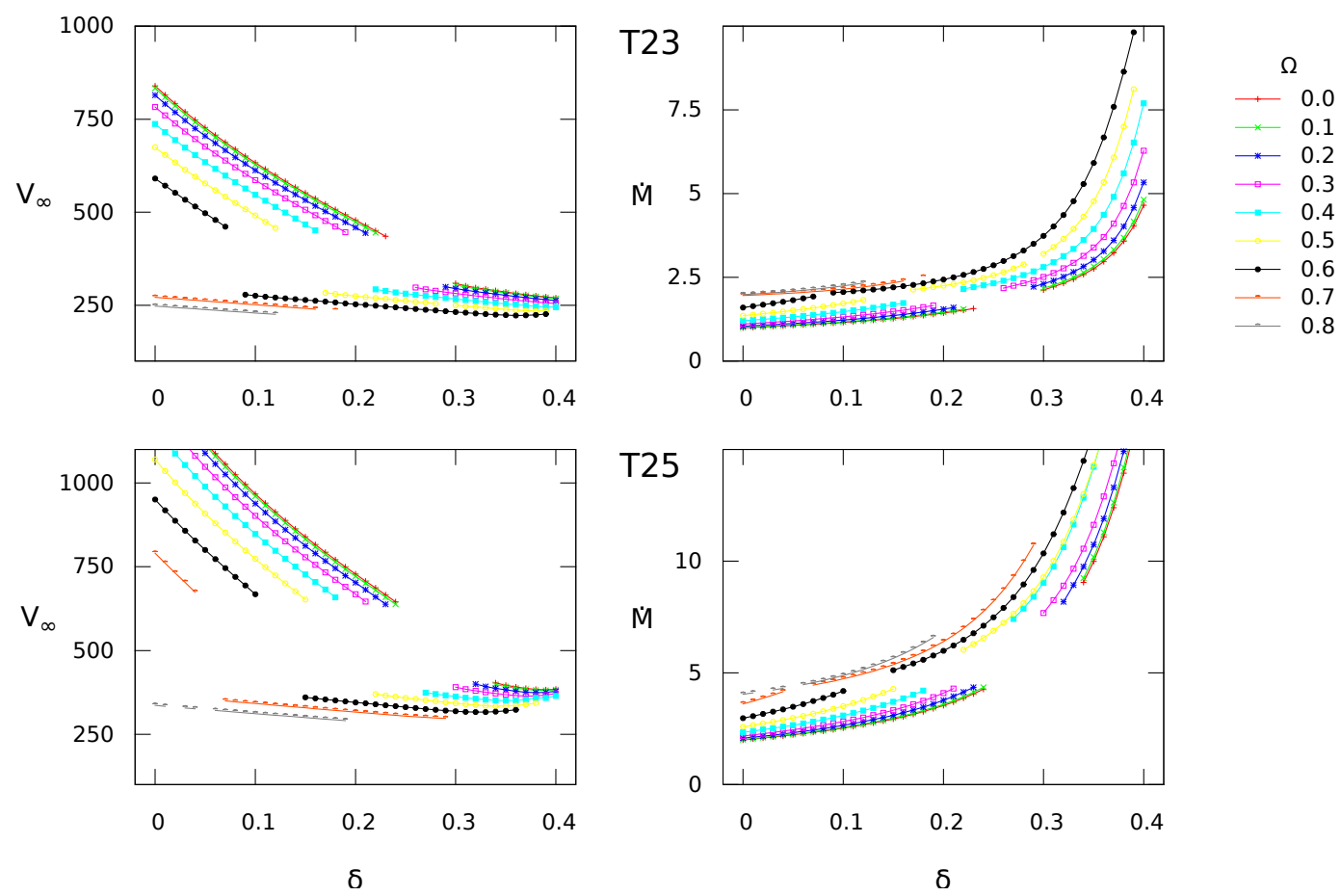

Figura 4.9. Los modelos base T23 y T25 recuperan la forma habitual al adoptar valores más bajos del parámetro $k$. Los parámetros en estos modelos son: para T23, $k=0.20$ y $\alpha=0.5$, mientras que para T25, $k=0.20$ y $\alpha=0.55$.

\subsubsection{La pendiente de la tasa de pérdida de masa y el pará- metro $k$}

El panel de la derecha y abajo de la figura 4.5, que presenta la dependencia de la tasa de pérdida de masa con el parámetro $\delta$ para el modelo base T15, muestra un comportamiento diferente en comparación con las figuras análogas para T13, y las correspondientes a T17 y T21 (figura 4.6). En estos últimos casos, $\dot{M}$ aparece como una función monótonamente creciente de $\delta$. Es decir, un aumento en la ionización produce un incremento en la tasa de pérdida de masa. Sin embargo, el modelo T15 muestra una inversión de ese comportamiento, especialmente para la solución lenta a bajas tasas rotacionales. En esos modelos, la tasa de pérdida de masa decae al aumentar la ionización.

La diferencia entre el modelo T15 y los otros modelos base radica en la elección del parámetro $k$, como puede comprobarse en la figura 4.10. Esta figura contiene tres pares de paneles, correspondiendo cada par, a un valor diferente del parámetro $k$. Cada curva se obtiene para una dada tasa rotacional (cuyo valor se indica en la referencia), como se hizo en la figura 4.2 y similares.

La figura 4.10 muestra que, dependiendo del valor del parámetro $k$, la relación entre la tasa de pérdida de masa y la ionización cambia completamente, invirtiendo su pendiente, y que eso se verifica tanto en el régimen lento como en el rápido.

La pendiente negativa en la dependencia de $\dot{M}$ con $\delta$ para bajos valores de $k$ es consecuencia de una competencia entre la distribución de ionización en el gas y su 

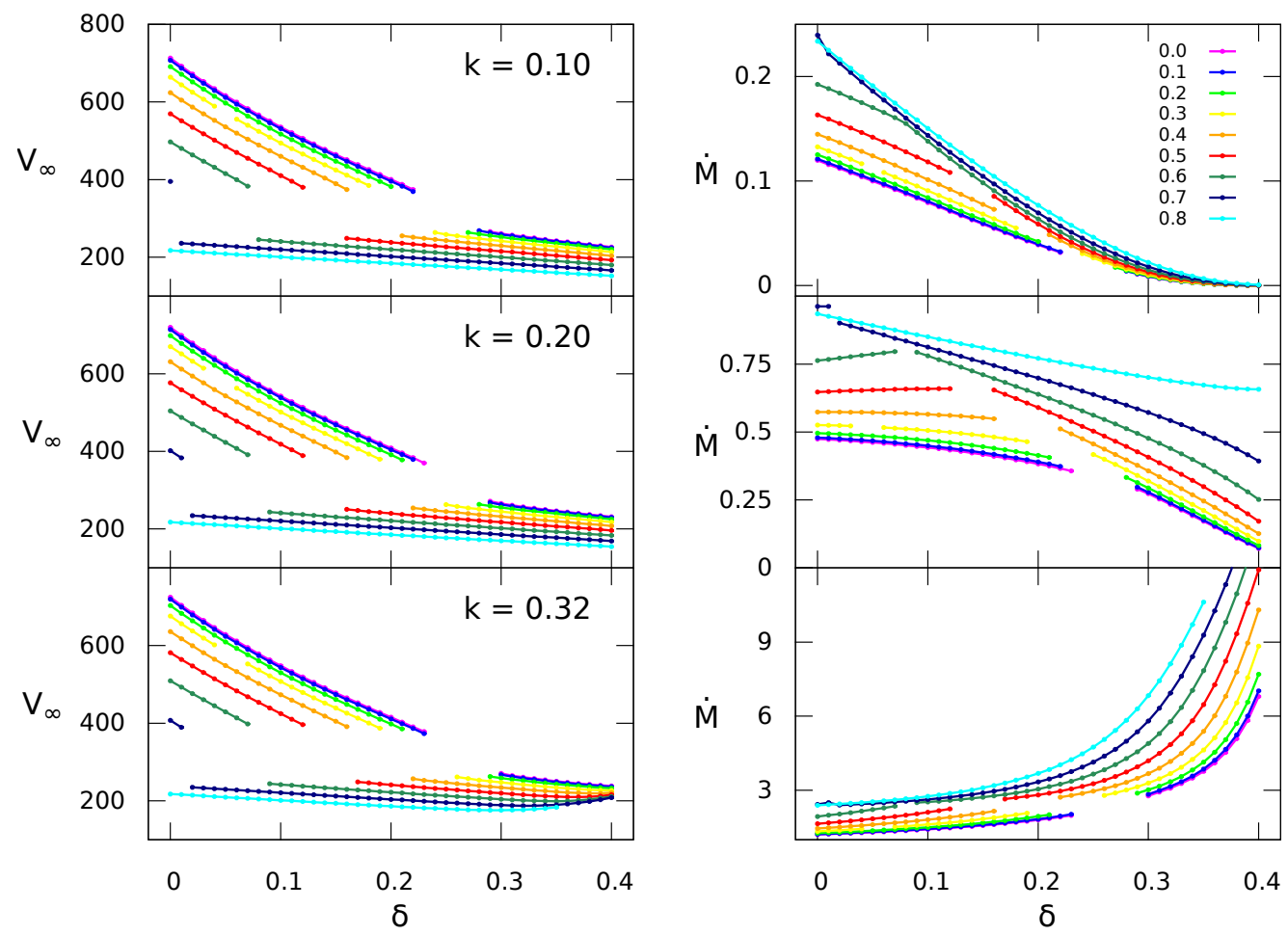

Figura 4.10. Velocidades terminales en $\mathrm{km} \mathrm{s}^{-1}$ (columna izquierda) y tasas de pérdida de masa en unidades de $10^{-6} M_{\odot}$ año ${ }^{-1}$ (columna derecha) en función de $\delta$ para diferentes valores del parámetro $\Omega$ (referenciados en el panel de la derecha). Cada par de paneles fue calculado para un parámetro $k$ diferente $(0.10,0.20$ y 0.32$)$. Todos los modelos usan los parámetros para el modelo base T19 con $\alpha=0.5$. Nótese la brecha cuya ubicación y ancho dependen de $\delta$ y $\Omega$. La relación entre $\dot{M}$ y $\delta$ se invierte completamente entre el panel superior y el inferior, mientras que $v_{\infty}$ permanece siempre con la misma tendencia.

capacidad para ganar momento. Un valor mayor de $k$ origina un viento más denso, con mayor tasa de pérdida de masa. Sin embargo, el dominio de $k$ puede ser mermado si se considera, simultáneamente, un incremento en la ionización. Esto se debe a que la ionización reduce la capacidad del gas de sustraer momento del campo de radiación.

En el régimen lento, si $k=0.20$ y $\Omega=0.4$, que es uno de los casos del panel intermedio en la figura 4.10 (línea de color anaranjado), el modelo con $\delta=0.3$ predice el doble de pérdida de masa que el modelo con $\delta=0.4$.

Este comportamiento también puede observarse en la relación entre $\dot{M}$ y $\delta$ (panel derecho) de la figura 4.11 del modelo base T19. Los valores bajos de $k$ producen curvas con pendientes negativas suaves tanto en los regímenes rápido como lento.

Sin embargo, para valores de $k$ justo por encima de 0.26 , la pendiente se vuelve positiva. Para este modelo base, un valor de $k=0.26$ produce que $\dot{M}$ sea casi insensible a variaciones en la ionización, dentro del régimen lento. Para el régimen rápido, se mantiene un valor casi constante para $\dot{M}$ para un parámetro $k$ levemente menor, de alrededor de 0.24 . 

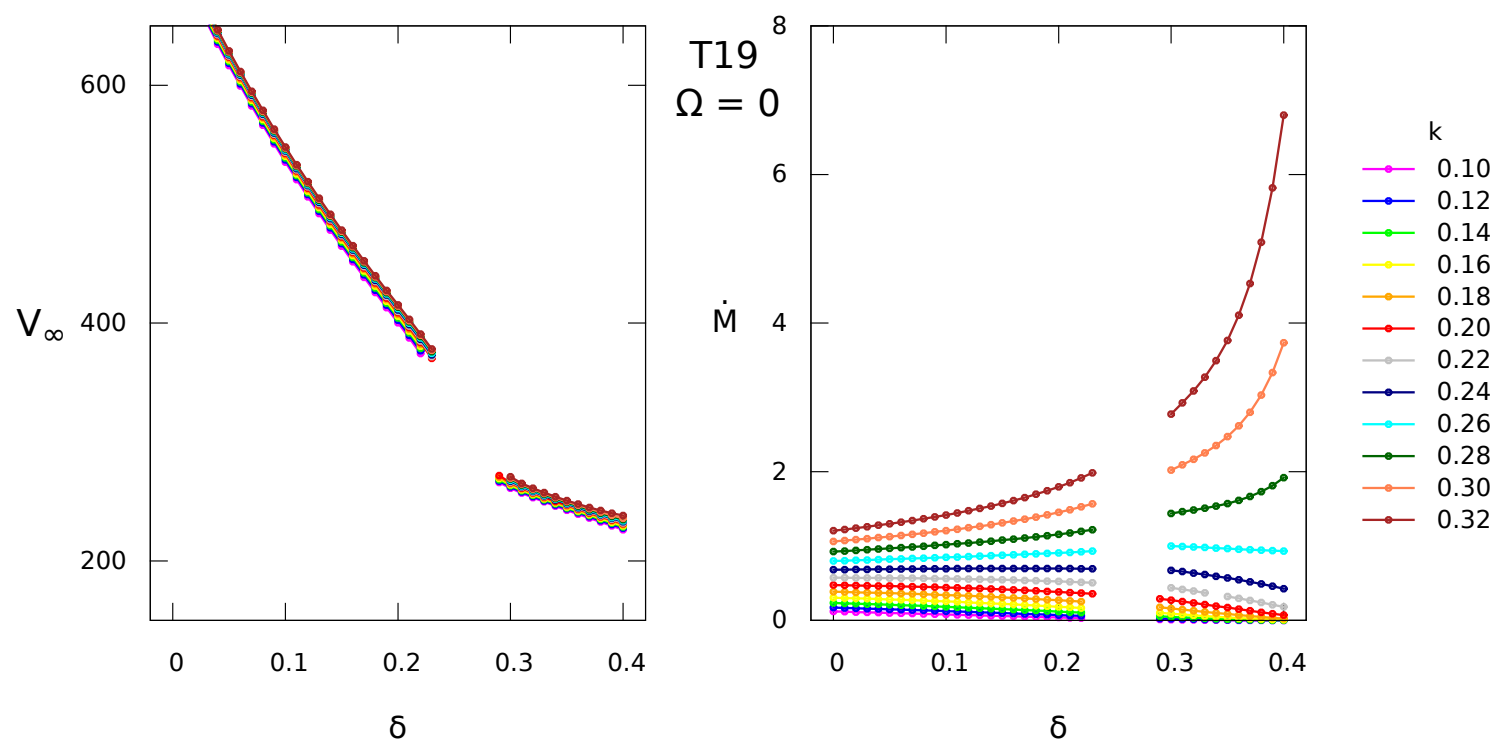

Figura 4.11. Gráfico similar a la figura 4.8 pero para el modelo base T19. Se confirma, nuevamente, que la velocidad terminal no depende de la ionización del medio, representada por el parámetro $\delta$. La tasa de pérdida de masa varía fuertemente con la ionización dependiendo del parámetro $k$. Para el valor $k=0.26$, la tasa de pérdida de masa es prácticamente insensible frente a variaciones en la ionización.

En estos modelos se ha producido un balance entre la reducción de absorción de momento debida a la ionización y la cantidad de momento transferido vía el número efectivo de líneas en condiciones de absorber.

Una consecuencia interesante de este balance entre los parámetros $k$ y $\delta$, es la posibilidad de ajustar el valor de $\mathrm{v}_{\infty}$ manteniendo $\dot{M}$ casi constante, por medio de una elección adecuada de un par de los parámetros $k$ y $\delta$ de la aceleración radiativa.

\subsection{Influencia del parámetro $\alpha$ en los dominios}

El parámetro $\alpha$, que es el parámetro de la fuerza de radiación que se corresponde con la pendiente en la distribución de intensidades de línea, produce una influencia sobre las soluciones, que es diferente a los efectos del parámetro $k$. La figura 4.12 muestra la dependencia de las soluciones con $\alpha$, en el mismo modo que para los otros parámetros. Estos modelos corresponden al modelo base T19, sin rotación y con un valor fijo de $k$ $=0.2$. A diferencia de lo que sucede a las soluciones respecto al parámetro $k$, en este caso, $v_{\infty}$ se encuentra fuertemente controlada por $\alpha$. Esto puede observarse en el panel izquierdo de la figura 4.12 en el que, los cambios en $\alpha$ originan curvas bien separadas en el espacio de $\delta$. Un aumento en los valores de $\alpha$ produce un crecimiento significativo en $\mathrm{v}_{\infty}$. Este comportamiento es prominente en el caso del régimen rápido, mientras que es moderado en el régimen lento.

Por otra parte, para bajos valores de $\alpha$, la relación entre $\dot{M}$ y $\delta$ presenta gradientes suaves. En los modelos calculados, existe un valor de $\alpha$ para el cual los valores de 

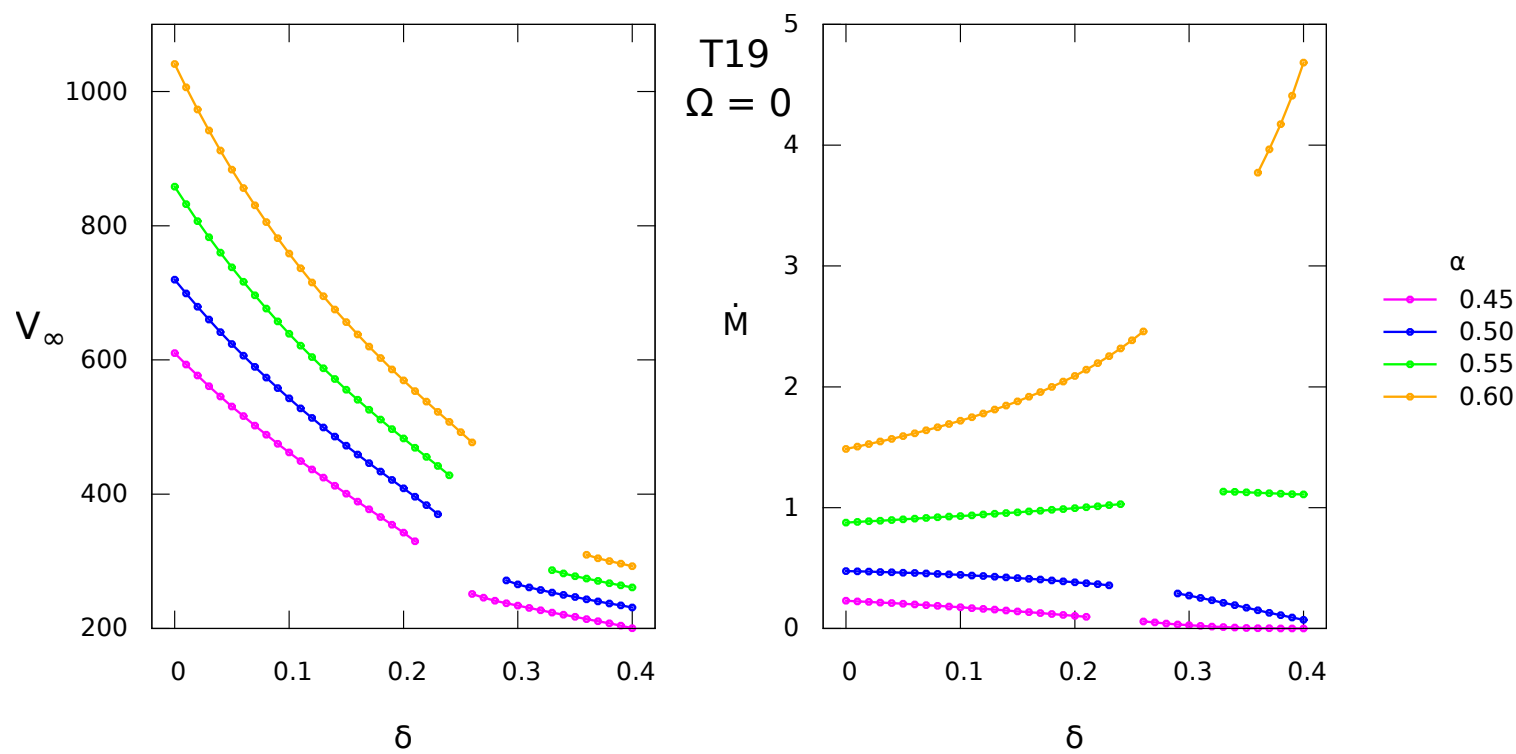

Figura 4.12. Velocidades terminales en $\mathrm{km} \mathrm{s}^{-1}$ (columna izquierda) y tasas de pérdida de masa en unidades de $10^{-6} M_{\odot}$ año ${ }^{-1}$ (columna derecha) en función de $\delta$ para diferentes valores del parámetro $\alpha$ (referenciados a la derecha). Los resultados mostrados corresponden al modelo base T19 con $k=0.2$ y $\Omega=0$.

$\dot{M}$ permanecen casi constantes, aún para valores muy altos de $\delta$. De acuerdo a la figura 4.12, este valor es $\alpha \sim 0.55$. Por lo tanto, podrían modificarse las condiciones de ionización sin que esos cambios se reflejaran en la tasa de pérdida de masa. Este es un resultado semejante al citado para $k=0.26$ pero, en este caso, involucrando al parámetro $\alpha$.

En el régimen lento, si se consideran valores altos simultáneos de $\alpha$ y de $\delta$ se producen pendientes pronunciadas en la relación $\dot{M}-\delta$. Entonces, en estos modelos, se genera un rápido incremento en el flujo del viento a medida que el material se ioniza.

Nuevamente, como en el caso de la sensibilidad de $\dot{M}$ con $k$ (sección 4.4.1), los valores de $\alpha$ deberían mantenerse acotados para evitar que las tasas de pérdida de masa sean demasiado altas, especialmente en el régimen lento. Este límite superior para $\alpha$ se encuentra próximo a 0.55 .

De acuerdo a Puls et al. (2000), como se menciona en la sección 2.2.7, el parámetro $\alpha$ es la pendiente de la función distribución de intensidad de líneas. El valor de $\alpha$ sería $2 / 3$ si todas las líneas se originaran en iones hidrogénicos y fueran líneas de resonancia (despreciando los efectos de excitación en niveles más altos). Este valor debería ser considerado como un límite superior en el caso de las supergigantes B.

También, si se interpreta a $\alpha$ como el cociente entre líneas ópticamente gruesas respecto al total de líneas, resulta claro que los vientos de las supergigantes B no son ni 
totalmente ópticamente gruesos ni totalmente ópticamente delgados. Justamente, una situación intermedia parece ser la mejor descripción.

\subsubsection{La brecha y el parámetro $\alpha$}

Como se puede observar en la figura 4.12, tanto la ubicación como el ancho de la brecha dependen fuertemente del parámetro $\alpha$. La localización del centro de la brecha, en el espacio del parámetro $\delta$ se desplaza hacia valores más altos de $\delta$, cuando $\alpha$ crece. De la misma manera, el ancho de la brecha crece considerablemente ante cambios pequeños en $\alpha$.

\subsection{Análisis de la brecha}

La existencia de una brecha que separa las soluciones de tipo rápida de las lentas fue omitida por los estudios previos, ya que todos ellos restringieron sus análisis a valores de $\delta$ acordes a los comúnmente adoptados por la teoría estándar de vientos impulsados por radiación. No obstante, Curé \& Rial (2004), en su análisis de la topología de la solución CAK, encontraron una bifurcación en las soluciones al considerar modelos con valores de $\Omega$ y $\delta$ no nulos. Estos autores también encontraron una región prohibida donde no pueden localizarse puntos críticos (figura 3.7). En el presente trabajo, la brecha encontrada se corresponde con una prolongación analítica de esa región prohibida.

A continuación, se enumeran algunas de las propiedades de la brecha:

- Ubicación de la brecha: En la figura 4.13(a) se muestra el valor medio de $\delta$ entre ambos bordes de la brecha, es decir $\delta_{\mathrm{m}}=\left(\delta_{\mathrm{b} 1}+\delta_{\mathrm{b} 2}\right) / 2$, en función de $\Omega$, brindando una referencia aproximada de la localización de la brecha en una escala de valores de $\delta$. Para todos los modelos base con $\mathrm{T}_{\text {eff }}<23000 \mathrm{~K}$, resulta claro que la brecha se desplaza hacia valores más bajos de $\delta$ a medida que $\Omega$ crece.

Además, la figura muestra que la brecha se encuentra centrada siempre en torno a un mismo valor de $\delta$, aún en el caso de $\mathrm{T}_{\text {eff }}$ muy diferentes. La excepción que muestra el gráfico (en línea de color negro) corresponde al modelo base T25, cuyos valores fueron calculados a partir de un parámetro $\alpha$ más alto $(\alpha=0.55)$ a los dados en la tabla 4.3 (en general, $\alpha=0.5$ ).

- Ancho de la brecha: En la figura 4.13(b) se ilustra la variación del ancho de la brecha, $\Delta \delta=\delta_{b 2}-\delta_{b 1}$, en escala de $\delta$, como función de $\Omega$. Puede notarse que el ancho disminuye a medida que $\Omega$ crece. En todos los modelos base, el ancho de la brecha muestra un comportamiento análogo.

Como se menciona en la sección 4.5.1, el parámetro $\alpha$ también modifica el ancho y la ubicación de la brecha en el espacio $\delta$, como puede verse en la figura 4.12 . Pequeños valores de $\alpha$ generan brechas más angostas y que están ubicadas hacia valores más bajos de $\delta$, mientras que grandes valores de $\alpha$ incrementan el ancho de esta zona. 


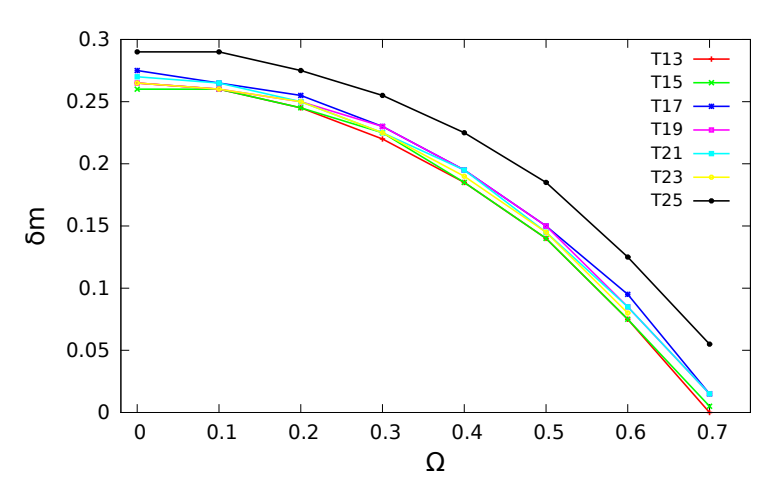

(a)

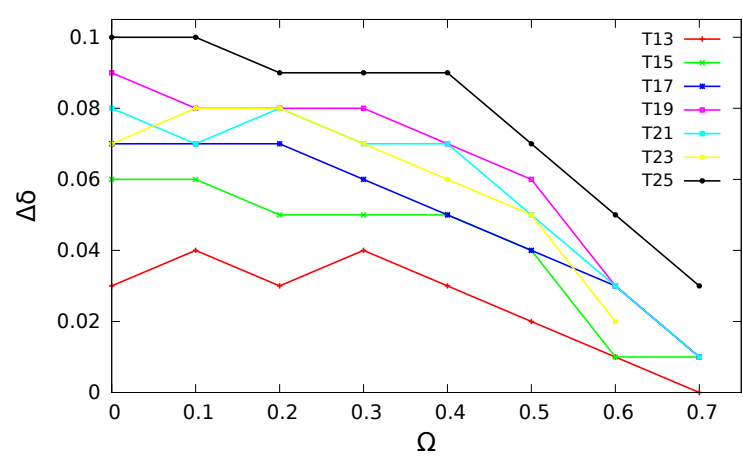

(b)

Figura 4.13. a) Ubicación de la brecha en escala de $\delta$, dada por el valor medio de $\delta$ entre los bordes de la brecha, $\delta_{\mathrm{m}}=\left(\delta_{\mathrm{b} 1}+\delta_{\mathrm{b} 2}\right) / 2$, en función de $\Omega$. Para todos los modelos base representados, la brecha se encuentra ubicada alrededor del mismo valor de $\delta_{\mathrm{m}}$, excepto para T25, cuyo valor de $\alpha$ es mayor (0.55). La ubicación de la brecha se desplaza a valores más pequeños de $\delta$, conforme la rotación aumenta.

b) Ancho de la brecha $\Delta \delta=\delta_{\mathrm{b} 2}-\delta_{\mathrm{b} 1}$, en escala de $\delta$, y en función de $\Omega$ para los modelos base representados. A mayor tasa rotacional, la brecha se vuelve más angosta.

- Altura en $\mathbf{v}_{\infty}$ de la brecha: La diferencia en valores de la velocidad terminal a ambos lados de la brecha $\left(v_{\infty f}-v_{\infty i}\right)$ es pequeña para modelos con temperaturas efectivas bajas. La diferencia en velocidad crece para modelos con valores mayores de $T_{\text {eff }} \mathrm{y} \Omega$. Este resultado puede verse en la figura 4.14(a).

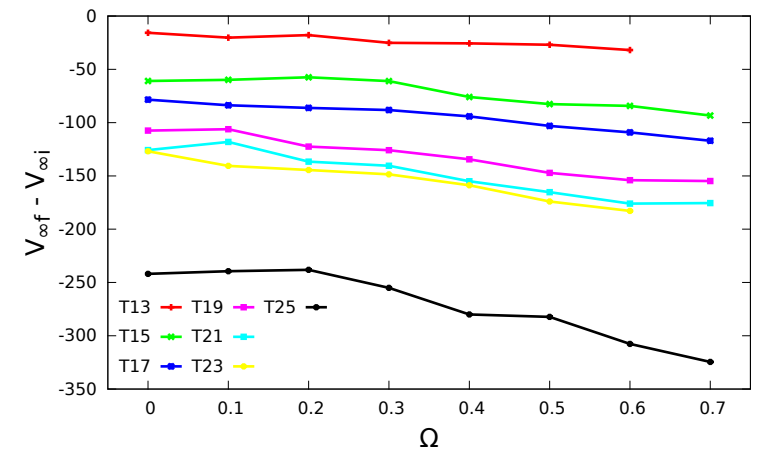

(a)

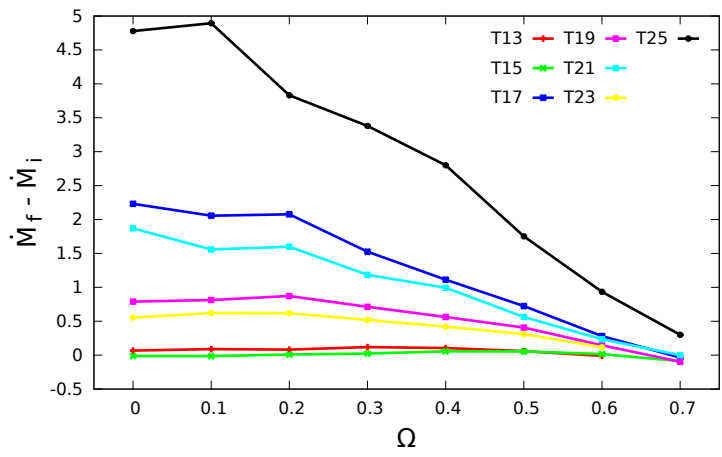

(b)

Figura 4.14. a) Altura de la brecha en escala de $\mathrm{v}_{\infty}\left(\right.$ en $\left.\mathrm{km} \mathrm{s}^{-1}\right)$, entre los bordes de la brecha en función de $\Omega$, para los modelos base indicados en el gráfico.

b) Altura de la brecha en escala de $\dot{M}$ (en $10^{-6} \mathrm{M}_{\odot}$ año $\left.{ }^{-1}\right)$, entre los bordes de la brecha, en función de $\Omega$.

- Altura en $\dot{M}$ de la brecha: En cambio, como puede apreciarse en la figura 4.14(b), la diferencia de tasa de pérdida de masa disminuye a medida que la tasa rotacional $\Omega$ es incrementada. Este salto en $\dot{M}$ es mayor para los subtipos más tempranos. En el gráfico las curvas presentan oscilaciones que pueden deberse a que los diferentes modelos base han sido calculados con distinto parámetro $k$, 
para el cual $\dot{M}$ depende fuertemente.

\subsubsection{Soluciones dentro de la brecha}

Resulta interesante indagar que sucede dentro de la brecha, es decir, cómo se comporta un modelo de viento cuyos parámetros, en especial $\delta$, lo ubican en la región intermedia entre los dominios rápido y lento. Este análisis no puede llevarse a cabo con HYDWIND, ya que este código resuelve las ecuaciones hidrodinámicas estacionarias. Madura et al. (2007) han indagado en una región del espacio de los parámetros semejante (sección 3.3.3), pero limitándose al caso de valores pequeños de $\delta$, es decir, en la región de la brecha situada entre el dominio de la solución rápida y el correspondiente a la solución $\Omega_{\text {lenta }}$.

Siendo la brecha una región de transición entre dos regímenes bien establecidos, en su interior podrían darse diferentes circunstancias. Una posibilidad es que el viento alcance un estado estacionario completamente diferente a la situación de los regímenes hidrodinámicos a ambos lados de la brecha. Otra posibilidad es que, en su interior, haya un estado caótico dependiente del tiempo. Debido a que la brecha separa dos regímenes diferentes, uno rápido y otro lento, podría esperarse que, dentro de la misma, haya una transición entre uno y otro régimen.

Para poder estudiar el estado hidrodinámico del viento bajo estas condiciones, se ha usado el código ZEUS-3D (Clarke 1996, 2010), que es un código dependiente del tiempo. Con este código se ha probado el modelo base T19, usando las mismas hipótesis que fueron aplicadas a HYDWIND (es decir, cálculos 1-D y fuerzas puramente radiales). Se usaron tres conjuntos de parámetros representativos de modelos en los dominios rápido $(\delta=0.07)$, lento $(\delta=0.33)$ e interior a la brecha $(\delta=0.26)$.

Es importante mencionar que el código ZEUS-3D es capaz de reproducir resultados similares a los encontrados previamente con HYDWIND en los dominios bien establecidos. Sin embargo, por ser un código que trata las ecuaciones hidrodinámicas dependientes del tiempo (ecuación 2.10), puede usarse para proveer una posible solución de las mismas, en el interior de la brecha.

La figura 4.15 muestra los campos de velocidades derivados con ZEUS-3D, en el modelo base T19, en función de $u=-R_{*} / r$, para una solución rápida $(\delta=0.07$, línea verde), una solución de tipo $\delta$-lenta $(\delta=0.33$, línea azul), y una solución para las condiciones interiores a la brecha ( $\delta=0.26$, línea roja).

Esta última solución presenta una brusca desaceleración, demostrando que, en el interior de la brecha, existe una solución estacionaria con la estructura de una ley de velocidades de tipo quiebre o plateau.

Como se menciona en la sección 2.6.6, las soluciones con estructura de quiebre han sido propuestas por Cranmer \& Owocki (1996) para explicar la formación de componentes discretas en absorción (DACs) en estrellas de tipo espectral O.

Para distinguir cada uno de los regímenes estudiados en el viento de las supergigantes B, es necesario buscar características observables que sirvan para diagnosticar a qué dominio de soluciones pertenece. En los capítulos siguientes se evalúan algunas líneas espectrales como posibles candidatos para este fin. Es necesario, entonces, resolver el transporte de radiación a través de medios en movimientos, con las propie- 


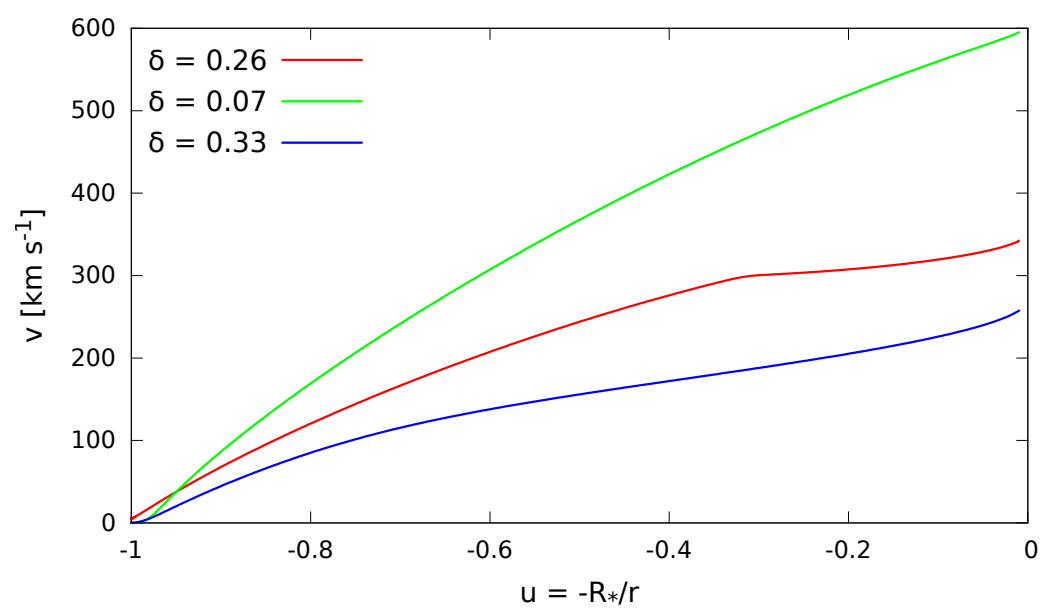

Figura 4.15. Campo de velocidades calculado con el código ZEUS-3D para un conjunto de parámetros que corresponden a: un modelo en el dominio rápido (línea verde), un modelo en el dominio lento (línea azul) y un modelo dentro de la brecha (línea roja). Nótese que la solución correspondiente a la brecha muestra una estructura tipo quiebre o plateau (meseta).

dades hidrodinámicas de las soluciones estudiadas, para poder avanzar en el análisis propuesto. 
4. Dominios de las soluciones de vientos en rotación impulsados por radiación 


\section{Capítulo 5}

\section{El transporte de radiación en medios en movimiento}

Resumen del capítulo: Se desarrollan los conceptos básicos del transporte de radiación en medios en movimiento, en la aproximación de Sobolev con y sin la contribución del continuo, y en el marco de referencia del fluido. Se introducen los códigos actuales de cálculo de atmósfera y se describe con más detalle el código FASTWIND, ya que éste es empleado directamente en esta tesis. Se desarrolla el tratamiento de las inversiones de poblaciones en la aproximación de Sobolev con continuo y en el marco de referencia del fluido. Se presenta la manera en que estos desarrollos fueron implementados en el código FASTWIND como parte del trabajo de esta tesis. En el caso en que el lector se encuentre familiarizado con el tratamiento del transporte de radiación y desee acceder directamente al trabajo desarrollado en esta tesis, se le sugiere comenzar la lectura en la sección 5.1.2.

\subsection{Transporte de radiación en vientos estelares}

La resolución simultánea de las ecuaciones 2.3, 2.78 y 2.19, permiten obtener una estratificación detallada de la estructura hidrodinámica de un viento estelar; es decir el comportamiento de la velocidad y la densidad en cada punto del medio, en el marco de las hipótesis planteadas por la teoría estándar de vientos impulsados por radiación.

Para realizar la comparación entre las características espectrales observables del viento de las estrellas y las predicciones de la teoría, es necesario además, evaluar el transporte de la radiación que proviene del interior de la estrella, que emerge de la fotosfera y finalmente atraviesa el viento, cuyas propiedades estarán dadas por las soluciones encontradas. La ecuación de transporte radiativo debe resolverse, entonces, para medios en movimiento, en los cuales el corrimiento Doppler de las frecuencias tiene gran importancia debido a la expansión del fluido. Las condiciones básicas de la teoría para estas ecuaciones son las planteadas en la sección 2.1.1, que implican esencialmente un sistema estacionario, homogéneo y en simetría esférica. Por otra parte, se debe resolver el transporte de radiación tanto en el continuo como en las líneas espectrales, lo cual requiere la evaluación simultánea de las ecuaciones de equilibrio estadístico para obtener las poblaciones en NLTE de los iones involucrados.

De acuerdo a Rybicki \& Hummer (1978), la ecuación de transporte en una línea 
( $\sin$ continuo) para un átomo de dos niveles en un medio en movimiento 3-D, es de la forma:

$$
\boldsymbol{n} \cdot \nabla I(\boldsymbol{r}, \boldsymbol{n}, \nu)=-\kappa(\boldsymbol{r}) \phi\left[\nu-\frac{\nu_{0}}{c} \boldsymbol{n} \cdot \boldsymbol{v}(\boldsymbol{r})\right][I-S],
$$

donde $I(\boldsymbol{r}, \boldsymbol{n}, \nu)$ es la intensidad específica en el punto $\boldsymbol{r}$ con la dirección dada por el versor $\boldsymbol{n}$, para una frecuencia $\nu$. Esta intensidad específica atraviesa un medio que se desplaza con una velocidad arbitraria $\boldsymbol{v}(\boldsymbol{r})$ y cuya función fuente, considerada isotrópica, es $S(\boldsymbol{r}, \nu)$. Despreciando la emisión estimulada, la opacidad integrada de línea tiene la forma:

$$
\kappa(\boldsymbol{r})=\frac{h \nu_{0}}{4 \pi} B_{l u} n_{l}(\boldsymbol{r}),
$$

siendo $\nu_{0}$, la frecuencia en el centro de la línea, $\mathrm{B}_{l u}$, el coeficiente de Einstein para la absorción correspondiente a la transición entre el nivel inferior $l(l \equiv l o w)$ y el superior $u(u \equiv u p) \mathrm{y}_{l}$, la población del nivel inferior de la transición en cuestión. La ecuación 5.1 también depende del perfil de absorción de la línea $\phi(\boldsymbol{r}, \nu)$, donde:

$$
\int_{0}^{\infty} \phi(\boldsymbol{r}, \nu) d \nu=1
$$

Si se adopta la hipótesis de redistribución completa, la función fuente toma la forma:

$$
S(\boldsymbol{r})=[1-\epsilon(\boldsymbol{r})] \bar{J}(\boldsymbol{r})+\epsilon(\boldsymbol{r}) B(\boldsymbol{r}),
$$

donde $\epsilon(\boldsymbol{r})$ mide la relación de dominio en la creación de fotones, entre los procesos de dispersión y los que producen fotones a expensas de la energía térmica del gas. $B(\boldsymbol{r})$ es la función de Planck a la temperatura electrónica local en $\boldsymbol{r}$. La intensidad media o momento de orden cero del campo de radiación, integrada en frecuencia $\square$ es:

$$
\bar{J}(\boldsymbol{r})=\frac{1}{4 \pi} \int d \omega \int_{0}^{\infty} \phi\left[\nu-\frac{\nu_{0}}{c} \boldsymbol{n} \cdot \boldsymbol{v}(\boldsymbol{r})\right] I(\boldsymbol{r}, \boldsymbol{n}, \nu) d \nu,
$$

la cual está integrada en el ángulo sólido d $\Omega$ en torno a la dirección $\boldsymbol{n}$.

Por otra parte, el vector flujo integrado sobre las frecuencias, es el primer momento del campo de radiación, y puede calcularse de la siguiente forma:

$$
\overline{\boldsymbol{H}}(\boldsymbol{r})=\frac{1}{4 \pi} \int d \omega \boldsymbol{n} \int_{0}^{\infty} \phi\left[\nu-\frac{\nu_{0}}{c} \boldsymbol{n} \cdot \boldsymbol{v}(\boldsymbol{r})\right] I(\boldsymbol{r}, \boldsymbol{n}, \nu) d \nu .
$$

Para visualizar la geometría del problema, se puede usar la figura 2.2 que localiza un punto en el viento y que muestra que se cumple la relación:

$$
\boldsymbol{r}=\boldsymbol{r}_{\mathbf{0}}+l \boldsymbol{n}
$$

Usando esta nomenclatura, la ecuación 5.1 tiene como solución formal:

\footnotetext{
${ }^{1}$ las cantidades integradas en frecuencia se indican con una barra superior, a menos que se lo indique expresamente.
} 


$$
\begin{aligned}
I(\boldsymbol{r}, \boldsymbol{n}, \nu)= & \int_{0}^{R} d l \kappa(\boldsymbol{r}-\boldsymbol{n l}) \phi\left[\nu-\frac{\nu_{0}}{c} \boldsymbol{n} \cdot \boldsymbol{v}(\boldsymbol{r}-\boldsymbol{n l})\right] S(\boldsymbol{r}-\boldsymbol{n l}) \\
& \times \exp \left\{-\int_{0}^{l} d l^{\prime} \kappa\left(\boldsymbol{r}-\boldsymbol{n} \boldsymbol{l}^{\prime}\right) \phi\left[\nu-\frac{\nu_{0}}{c} \boldsymbol{n} \cdot \boldsymbol{v}\left(\boldsymbol{r}-\boldsymbol{n} l^{\prime}\right)\right]\right\} \\
& +I_{\nu}^{i n c} \exp \left\{-\int_{0}^{R} d l^{\prime} \kappa\left(\boldsymbol{r}-\boldsymbol{n} l^{\prime}\right) \phi\left[\nu-\frac{\nu_{0}}{c} \boldsymbol{n} \cdot \boldsymbol{v}\left(\boldsymbol{r}-\boldsymbol{n} l^{\prime}\right)\right]\right\},
\end{aligned}
$$

donde $\mathrm{R}$ es la extensión total de la capa de variable $l$, y la intensidad incidente $I_{\nu}^{\text {inc }}$ se supone que ha sido especificada. Esta intensidad puede ser nula, si la dirección $\boldsymbol{n}$ del rayo no incide en la superficie estelar, o tomar un valor $I_{\nu}^{c}$, si se trata de un rayo que sí lo hace ("c" 三 core).

Hay varias maneras para obtener las soluciones finales para el campo de radiación que surgen de evaluar el transporte de radiación a partir de la ecuación 5.8. En esta tesis sólo se consideran las siguientes alternativas: la aproximación de Sobolev en el transporte de línea pura (sección 5.1.1), de línea con continuo (sección 5.1.2) y el transporte en el comoving frame (sección 5.1.3). Estos desarrollos, que son necesarios para abordar los resultados que se presentan a lo largo de este trabajo de tesis, serán compendiados de manera muy concisa, resaltando sus resultados finales y evitando así, un tratamiento demasiado extenso de temas que no fueron elaborados por el autor de esta tesis.

\subsubsection{Transporte de radiación en la aproximación de Sobolev}

Las condiciones de validez de la aproximación de Sobolev se encuentran en la sección 2.2.3, al igual que sus ideas principales. En esta sección se reunirán sólo los resultados fundamentales respecto a la resolución del transporte de energía en esta aproximación, ya que estos son relevantes para los desarrollos que se llevan a cabo en este trabajo.

A continuación se investiga el comportamiento de la intensidad en las proximidades de las regiones de resonancia introducidas en la sección 2.2.3.

Rybicki \& Hummer (1978) propone desarrollar las velocidades planteadas en la ecuación 5.8 hasta el primer orden en $l$, como:

$$
v_{i}(\boldsymbol{r}-\boldsymbol{n l})=v_{i}(\boldsymbol{r})-\sum_{j} n_{j} \frac{\partial v_{i}(\boldsymbol{r})}{\partial r_{j}} l,
$$

donde se ha usado una notación tensorial. Por lo tanto, el producto escalar con el versor, resulta:

$$
\boldsymbol{n} \cdot \boldsymbol{v}(\boldsymbol{r}-\boldsymbol{n} l)=\boldsymbol{n} \cdot \boldsymbol{v}(\boldsymbol{r})-\mathcal{Q}(\boldsymbol{r}, \boldsymbol{n}) l
$$

en el cual $\mathcal{Q}$ es:

$$
\mathcal{Q}(\boldsymbol{r}, \boldsymbol{n})=\sum_{i} \sum_{j} n_{i} n_{j} \frac{\partial v_{i}}{\partial v_{j}}=\frac{d v_{l}}{d l},
$$


cuyo valor ya se ha obtenido (similar al caso de la ecuación 2.43 de la sección 2.2.3), para el caso de simetría esférica (para su demostración, ver el apéndice .1), y es:

$$
\mathcal{Q}=\mu^{2} \frac{\partial v}{\partial r}+\left(1-\mu^{2}\right) \frac{v}{r}
$$

La opacidad $\kappa$ y la función fuente $S$ son funciones que cambian muy lentamente a lo largo de una región de resonancia, por lo que pueden ser consideradas constantes y extraídas de las integrales de la ecuación 5.8. Introduciendo la expresión 5.10 en dicha ecuación, y realizando un cambio de variables adecuado (ver los detalles en Rybicki \& Hummer 1978), la ecuación 5.8 puede simplificarse, quedando para la intensidad saliente, $I_{\nu}^{s a l}$, la forma final:

$$
I_{\nu}^{s a l}=I_{\nu}^{i n c} e^{-\tau}+S\left(1-e^{-\tau}\right),
$$

con la profundidad óptica total definida como:

$$
\tau=\kappa \frac{\Delta l}{\Delta \nu}=\frac{\kappa c}{\nu_{0}|\mathcal{Q}|}
$$

que es la profundidad óptica de Sobolev, definida en la ecuación 2.44.

La ecuación 5.13 muestra la simplicidad de la solución, en el caso de la aproximación de Sobolev. De acuerdo a esta, la intensidad permanece constante a lo largo de un rayo hasta que encuentra una región de resonancia; entonces se modifica en ese entorno, para luego emerger y permanecer constante hasta escapar del medio o encontrar una nueva región de resonancia.

Para evaluar la intensidad media y el flujo (ecuaciones 5.5 y 5.6) es necesario calcular la integral en frecuencia de la intensidad pesada con la función perfil $\phi$ :

$$
\bar{I}(\boldsymbol{r}, \boldsymbol{n})=\int_{0}^{\infty} \phi\left[\nu-\frac{\nu_{0}}{c} \boldsymbol{n} \cdot \boldsymbol{v}(\boldsymbol{r})\right] I(\boldsymbol{r}, \boldsymbol{n}, \nu) d \nu,
$$

usando la ecuación 5.13 , puede obtenerse:

$$
\bar{I}(\boldsymbol{r}, \boldsymbol{n})=I_{\nu}^{i n c} \frac{1-e^{-\tau}}{\tau}+S\left[1-\frac{1-e^{-\tau}}{\tau}\right] .
$$

Las ecuaciones 5.13 y 5.16 son las ecuaciones fundamentales de la aproximación de Sobolev, aplicada al caso de redistribución completa. A partir de ellas se puede obtener $\bar{J}(\boldsymbol{r})$, reemplazando la intensidad incidente (ecuación 5.13) en la ecuación 5.5 y teniendo en cuenta que $I_{\nu}^{i n c}=I_{c}$ para los rayos que impactan en el disco estelar, es decir, que están contenidos en $\Omega_{c}$, el ángulo sólido que subtiende la estrella; y que $I_{\nu}^{i n c}=0$, en el caso de los rayos que no tocan la superficie estelar. Con esto se obtiene:

$$
\bar{J}(\boldsymbol{r})=\left[1-\beta_{s}(\boldsymbol{r})\right] S(\boldsymbol{r})+\beta_{c}(\boldsymbol{r}) I_{c}
$$

en la cual, las cantidades $\beta_{s}$ y $\beta_{c}$ se definen como: 


$$
\begin{gathered}
\beta_{s}(\boldsymbol{r})=\frac{1}{4 \pi} \int d \omega \frac{1-e^{-\tau}}{\tau} \\
y \\
\beta_{c}(\boldsymbol{r})=\frac{1}{4 \pi} \int_{\Omega_{c}} d \omega \frac{1-e^{-\tau}}{\tau},
\end{gathered}
$$

donde $\beta_{s}(\boldsymbol{r})$ es llamada probabilidad de escape, y es la probabilidad de que un fotón emitido pueda escapar de la región de resonancia sin ser absorbido o dispersado. Nótese que, en esta última, los límites de integración restringen los rayos que contribuyen a la intensidad media, a aquellos que intersectan la superficie estelar. Por esta razón, esta cantidad se interpreta como la probabilidad de que un fotón que escapa de la región de resonancia, impacte la superficie de la estrella.

Por su parte, $\beta_{c}(\boldsymbol{r})$ recibe el nombre de probabilidad de penetración. En el caso de simetría esférica, estas cantidades quedan definidas como:

$$
\begin{aligned}
& \beta_{s}(r)=\frac{1}{2} \int_{-1}^{+1} d \mu \frac{1-e^{-\tau}}{\tau} \\
& y \\
& \beta_{c}(r)=\frac{1}{2} \int_{\mu_{c}}^{+1} d \mu \frac{1-e^{-\tau}}{\tau} .
\end{aligned}
$$

Para el flujo se obtiene:

$$
\bar{H}(\boldsymbol{r})=\gamma_{c}(\boldsymbol{r}) I_{c}
$$

siendo

$$
\gamma_{c}(\boldsymbol{r})=\frac{1}{4 \pi} \int_{\Omega_{c}} d \omega \boldsymbol{n} \frac{1-e^{-\tau}}{\tau}
$$

que, para simetría esférica queda expresado de la siguiente manera:

$$
\bar{H}=\gamma_{c} I_{c}, \quad \text { donde } \quad \gamma_{c}=\frac{1}{2} \int_{\mu_{c}}^{+1} d \mu \mu \frac{1-e^{-\tau}}{\tau} .
$$

Finalmente, la función fuente, definida en la ecuación 5.4 queda expresada de la siguiente forma:

$$
S(\boldsymbol{r})=\frac{(1-\epsilon) \beta_{c} I_{c}+\epsilon B}{\epsilon+(1-\epsilon) \beta_{s}},
$$

en la cual, una de las cantidades más importantes a determinar en la aproximación de Sobolev, es el producto $\beta_{c} I_{c}$. 


\subsubsection{Transporte de radiación en la aproximación de Sobolev con continuo}

Para extender la aproximación de Sobolev para líneas espectrales, de modo de incorporar el continuo de radiación que actúa tanto dentro como fuera de la región de resonancia, puede usarse la metodología propuesta por Hummer \& Rybicki (1985), que se resume a continuación.

En el caso en que no hubiera contribución de fotones del continuo, la intensidad emergente y su valor integrado en frecuencia estarían dadas por las ecuaciones 5.13 y 5.16. respectivamente, obtenidas en la sección anterior, para las líneas espectrales.

$\mathrm{Al}$ incorporar la radiación del continuo puede hacerse una aproximación simple, despreciando su contribución en el interior de las regiones de resonancias. Supóngase que se analizan los cambios de la intensidad de la radiación a lo largo de un rayo. Mientras el rayo no alcance una región de resonancia, pueden calcularse estos cambios resolviendo una ecuación de transporte simple para la radiación del continuo:

$$
\frac{d I}{d l}=-\kappa_{C}\left(I-S_{C}\right)
$$

siendo $\kappa_{C}$ y $\mathrm{S}_{C}$, el coeficiente de absorción y la función fuente del continuo, respectivamente. De esa ecuación se obtiene como solución, una intensidad saliente $I_{\nu}^{\text {sal }}$. Cuando el rayo alcanza una región de resonancia, esa intensidad será la intensidad incidente $I_{\nu}^{\text {inc }}$, necesaria para evaluar las ecuaciones 5.13 y 5.16 dentro de la región de resonancia. La intensidad saliente de estas ecuaciones, luego de que la radiación ha interactuado con los átomos o iones presentes en la región de resonancia, será la nueva intensidad incidente en una nueva ecuación del tipo 5.24, para el resto de camino del rayo. Así sucesivamente puede resolverse, a través de varias regiones de resonancia, si es necesario.

Adoptando ese proceso, se desea incorporar ahora la radiación del continuo dentro de las regiones de resonancia. Por lo tanto, la ecuación de transporte a resolver debe vincular tanto los fenómenos de línea como los de continuo:

$$
\frac{d I}{d l}=-\kappa_{L} \phi(t)\left(I-S_{L}\right)-\kappa_{C}\left(I-S_{C}\right),
$$

en la que se ha definido la posición espacial $l=0$ y la coordenada de frecuencia $x=0$, en la posición de la región de resonancia. El argumento de la función perfil de línea es $t=x-l / \Delta l$, siendo $\Delta l$, la longitud de Sobolev, definida en la ecuación 2.45.

Para resolver la ecuación 5.25. Hummer \& Rybicki (1985) proponen que, en lugar de la intensidad $I(x, l)$ se defina una nueva intensidad $\tilde{I}(x, l)$ dada por la relación:

$$
\tilde{I}(x, l)=U_{C}(l) I(x, l)-\int_{0}^{l} U_{C}\left(l^{\prime}\right) \kappa_{C}\left(l^{\prime}\right) S_{C}\left(l^{\prime}\right) d l^{\prime},
$$

donde

$$
U_{C}(l)=\exp \left[\int_{0}^{l} \kappa_{C}\left(l^{\prime \prime}\right) d l^{\prime \prime}\right]
$$


La ecuación 5.26 tiene la forma de una solución formal aplicada a la radiación entrante $I(x, l)$ y saliente $\tilde{I}(x, l)$ si sólo hubiera procesos de continuo. Diferenciando esa ecuación y usando la ecuación 5.25 , puede encontrarse que $\tilde{I}(x, l)$ satisface que:

$$
\frac{d \tilde{I}}{d l}=-\kappa_{L} \phi(t)(\tilde{I}-\tilde{S})
$$

con

$$
\tilde{S}=U_{C} S_{L}-\int_{0}^{l} U_{C}\left(l^{\prime}\right) \kappa_{C}\left(l^{\prime}\right) S_{C}\left(l^{\prime}\right) d l^{\prime} .
$$

En la ecuación 5.28 puede verse que, en ausencia de línea $\left(\kappa_{L}=0\right), \tilde{I}$ es constante. Si se supone que, de acuerdo a la aproximación de Sobolev, $\kappa_{L}, \kappa_{C}, \mathrm{~S}_{L}$ y $\mathrm{S}_{C}$ cambian muy lentamente al atravesar la región de resonancia, éstas pueden ser extraidas de la integral, obteniendo a partir de la ecuación 5.29 , la siguiente expresión:

$$
\tilde{S}=S_{L}+\left(S_{C}-S_{L}\right)\left(1-e^{\kappa_{C} l}\right) .
$$

Así, la ecuación 5.26 tiene como solución a:

$$
\begin{aligned}
\tilde{I}(x, l)= & I^{i n c} e^{-\tau \Phi(t)}+S_{L}\left[1-e^{-\tau \Phi(t)}\right] \\
& +\left(S_{C}-S_{L}\right) \tau \int_{t}^{\infty} d x^{\prime} \phi\left(x^{\prime}\right) \exp \left[-\tau \int_{t}^{x^{\prime}} \phi\left(x^{\prime \prime}\right) d x^{\prime \prime}\right]\left[1-e^{-\beta \tau\left(x^{\prime}-x\right)}\right]
\end{aligned}
$$

en la cual

$$
\Phi(t)=\int_{t}^{\infty} d x^{\prime} \phi\left(x^{\prime}\right)
$$

En $l=0$, de acuerdo a la ecuación $5.26, \tilde{I}(x, 0)$ coincide con $I(x, 0)$, por lo cual la ecuación 5.31 da la variación en frecuencia de la intensidad verdadera en ese punto.

Para obtener la intensidad integrada $\bar{I}$ en $l=0$, se usa la ecuación 5.31 multiplicada por $\phi(x)$ e integrada sobre todas las frecuencias, la cual da:

$$
\bar{I}=I^{i n c} \frac{1-e^{-\tau}}{\tau}+S_{L}\left(1-\frac{1-e^{-\tau}}{\tau}\right)+\left(S_{C}-S_{L}\right) U(\tau, \beta),
$$

donde se ha designado a $\beta=\kappa_{C} / \kappa_{L}^{\prime}$, con $\kappa_{L}^{\prime}=\kappa_{L} / \Delta \nu_{D}$. La ecuación 5.33 es la generalización de la ecuación 5.13 a la cual se le suma una corrección proporcional a la diferencia entre $\left(S_{C}-S_{L}\right)$. Si ambas funciones fuentes son iguales, el término se anula y se recupera la situación previa. También se hace despreciable en el caso en que el continuo sea débil, es decir si $\beta \tau \ll 1$. La función $U(\tau, \beta)$ es llamada función de interacción y tiene la forma:

$$
U(\tau, \beta) \equiv \tau \int_{-\infty}^{\infty} d x \phi(x) \int_{-\infty}^{x} d x^{\prime} \phi\left(x^{\prime}\right) \exp \left[-\tau \int_{x}^{x^{\prime}} d u \phi(u)\right]\left\{1-\exp \left[-\beta \tau\left(x^{\prime}-x\right)\right]\right\}
$$


Para la intensidad media integrada $\bar{J}$ se debe integrar la ecuación 5.33 sobre todos los ángulos sólidos, lo cual da:

$$
\bar{J}=\frac{1}{4 \pi} \int d \omega I^{i n c} \frac{1-e^{-\tau}}{\tau}+S_{L}\left(1-\beta_{s}\right)+\left(S_{C}-S_{L}\right) \frac{1}{4 \pi} \int d \omega U(\tau, \beta),
$$

donde $\beta_{s}$ es, nuevamente, la probabilidad de escape de Sobolev (ecuación 5.18):

$$
\beta_{s}=\frac{1}{4 \pi} \int d \omega \frac{1-e^{-\tau}}{\tau}
$$

\subsubsection{Transporte de radiación en el comoving frame}

Las ecuaciones 5.1 o 5.25, que representan el transporte de energía radiativa a través del viento estelar, están escritas en el marco de referencia estacionario de un observador externo. Si se quisieran resolver estas ecuaciones, en una aproximación diferente a la de Sobolev, se debería tener en cuenta que las opacidades y las emisividades del material dependen fuertemente de la dirección de los fotones, es decir, de la variable angular. Esto se debe, especialmente, a los cambios en la frecuencia que surgen por efecto de los corrimientos Doppler producidos por el movimiento del material. Estos efectos acoplan estrechamente a las frecuencias con los ángulos, lo cual acarrea serias dificultades para resolver los términos de dispersión (scattering) de los fotones. Para evitar estas dificultades, es conveniente resolver el transporte de radiación en el marco de referencia del fluido o comoving frame (CMF).

El uso del CMF tiene numerosas ventajas respecto al marco de referencia del observador (Mihalas 1978), las que se resumen a continuación:

- En el CMF, tanto la opacidad como la emisividad del material se comportan como isotrópicas, es decir, no dependen de las variables angulares.

- Para representar a las funciones de redistribución, necesarias para estimar correctamente los efectos de la dispersión, pueden usarse las funciones simples calculadas para medios estáticos. En general, se hace uso de la aproximación de redistribución completa.

- En los cálculos de las integrales de la dispersión, sólo es necesario tener en cuenta a las frecuencias contenidas dentro del perfil de la línea, cuyo ancho es independiente de la ley de velocidades.

- En los flujos con simetría esférica puede usarse un sistema de coordenadas lagrangiano, cuya formulación es más simple que para el sistema euleriano.

Como desventaja, los campos de velocidad permitidos deben ser simples; especialmente restringidos a medios con campos de velocidades monótonamente crecientes o decrecientes.

Si un fotón tiene frecuencia $\nu$ en el marco del observador, su frecuencia en el CMF será $\nu_{0}=\nu(1-\mu v / c)$. De acuerdo a Mihalas (1978), la ecuación de transporte para geometría esférica en el CMF toma la forma: 


$$
\begin{aligned}
& \mu \frac{\partial I(r, \mu, \nu)}{\partial r}+\frac{\left(1-\mu^{2}\right)}{r} \frac{\partial I(r, \mu, \nu)}{\partial \mu}-\left(\frac{\nu_{0} v(r)}{c r}\right)\left[\left(1-\mu^{2}\right)+\mu^{2}\left(\frac{d \ln v}{d \ln r}\right)\right] \frac{\partial I(r, \mu, \nu)}{\partial \nu} \\
& =\eta(r, \nu)-\chi(r, \nu) I(r, \mu, \nu)
\end{aligned}
$$

donde $\eta$ y $\chi$ son la emisividad y la opacidad del material. En esta fórmula, todas las cantidades están medidas en el marco solidario al fluido.

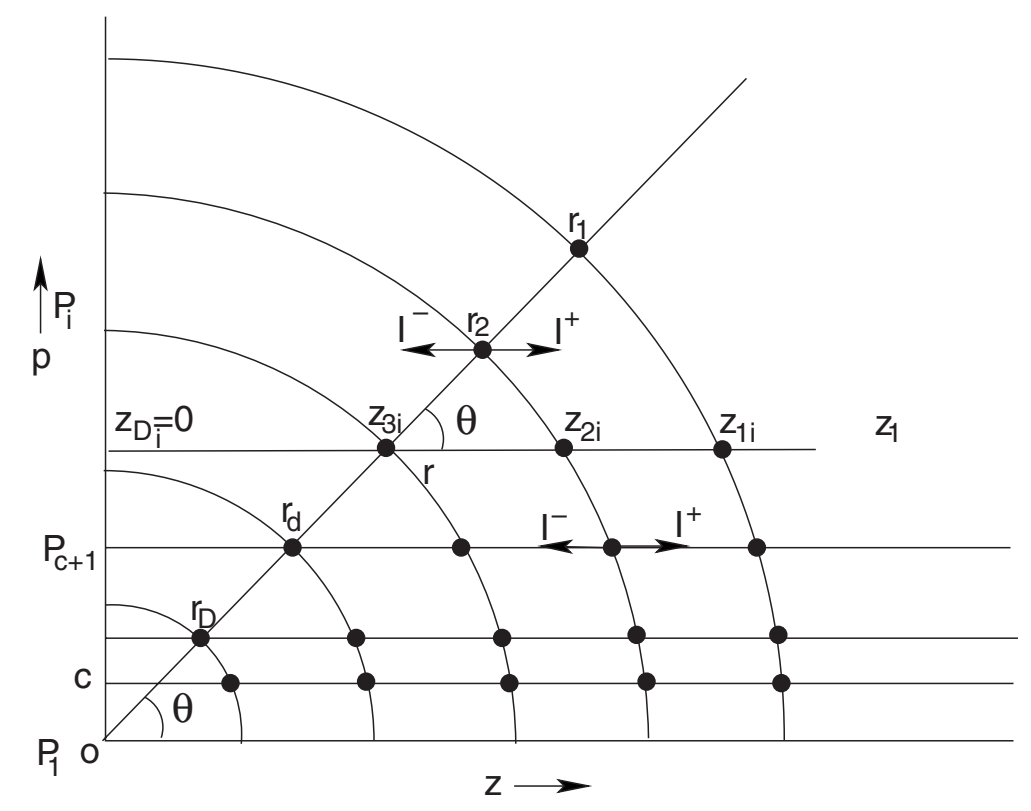

Figura 5.1. Sistema de coordenadas (p,z). Los valores de p designan la distancia de cada rayo horizontal al rayo central $(\mathrm{p}=0)$, es decir, a cada valor de un parámetro de impacto. Los parámetros de impacto son paralelos al rayo central que pasa por centro de simetría. Algún valor de $\mathrm{p}\left(\mathrm{p}=\mathrm{R}_{*}\right)$ coincide con el radio estelar, siendo que, para valores de $\mathrm{p}$ menores que este valor, todos los rayos impactan en la superficie estelar. A lo largo de cada rayo se mide la coordenada $z$, la cual es creciente en dirección hacia el observador (que se encuentra hacia la derecha del gráfico, en $z \rightarrow \infty$ ). La intensidad específica saliente es $\mathrm{I}^{+}$y apunta en el sentido creciente de $\mathrm{z}$, como se indica en la figura.

Es común reemplazar el sistema radial de coordenadas $(\mathrm{r}, \mu)$, por el sistema de coordenadas de parámetros de impacto $(\mathrm{p}, \mathrm{z})$, que puede verse en la figura 5.1. La relación de este sistema con el sistema en geometría esférica está dada por:

$$
\left\{\begin{array}{l}
r \equiv\left(p^{2}+z^{2}\right)^{1 / 2} \\
\mu \equiv z / r
\end{array}\right.
$$

En este sistema de coordenadas, a lo largo de un único rayo (p constante), la ecuación 5.37 puede dividirse en dos ecuaciones, una para la intensidad saliente $\left(I^{+}\right)$y otra, para la intensidad entrante $\left(I^{-}\right)$: 


$$
\pm\left[\frac{\partial I^{ \pm}(z, p, \nu)}{\partial z}\right]-\tilde{\gamma}(z, p)\left[\frac{\partial I^{ \pm}(z, p, \nu)}{\partial \nu}\right]=\eta(r, \nu)-\chi(r, \nu) I^{ \pm}(z, p, \nu),
$$

donde

$$
\tilde{\gamma}(z, p) \equiv\left[\frac{\nu v(r)}{c r}\right]\left[1-\mu^{2}+\mu^{2} \frac{d \ln v(r)}{d \ln r}\right] .
$$

Introduciendo la profundidad óptica $\mathrm{d} \tau(z, p, \nu)=-\chi(z, p, \nu) d z$, y las variables:

$$
\left\{\begin{array}{l}
U(z, p, \nu) \equiv \frac{1}{2}\left[I^{+}(z, p, \nu)+I^{-}(z, p, \nu)\right] \\
V(z, p, \nu) \equiv \frac{1}{2}\left[I^{+}(z, p, \nu)-I^{-}(z, p, \nu)\right]
\end{array}\right.
$$

se obtiene, de las ecuaciones 5.39, el sistema:

$$
\begin{aligned}
& {\left[\frac{\partial U}{\partial \tau}\right]+\gamma\left[\frac{\partial V}{\partial \nu}\right]=V} \\
& {\left[\frac{\partial V}{\partial \tau}\right]+\gamma\left[\frac{\partial U}{\partial \nu}\right]=U-S}
\end{aligned}
$$

donde $\gamma(z, p, \nu)=\tilde{\gamma}(z, p) \chi(z, p, \nu)$, y con la función fuente $\mathrm{S}(\mathrm{z}, \mathrm{p}, \nu)$ de la forma definida en la ecuación 5.4 , donde $\bar{J}(\mathrm{r})$ tiene la forma:

$$
\bar{J}(r)=\int_{\nu_{\min }}^{\nu_{\max }} d \nu \phi(\nu) \int_{0}^{1} d \mu U[z(r, \mu), p(r, \mu), \nu],
$$

con $\nu_{\min }$ y $\nu_{\max }$ elegidas para contener el perfil completo de la línea.

Las ecuaciones 5.42 deben resolverse con dos condiciones de borde y una condición inicial para la frecuencia:

(a) Borde externo: para $r=R_{*}$ no hay radiación entrante, $\mathrm{I}^{-}=0$. Entonces $\mathrm{U} \equiv \mathrm{V}$ y:

$$
\left[\frac{\partial U}{\partial \tau}\right]_{z_{\max }}+\gamma\left[\frac{\partial U}{\partial \nu}\right]_{z_{\max }}=U\left(z_{\max }, p, \nu\right)
$$

(b) Borde interno: En $\mathrm{z}=0$, se cumple $\mathrm{V}(0, \mathrm{p}, \nu) \equiv 0$, para los rayos que no intersectan la estrella, dando:

$$
\left[\frac{\partial U}{\partial \tau}\right]_{z=0}=0
$$

Para los rayos que intersectan la estrella, se aplica la aproximación de difusión (sección 2.5 de Mihalas 1978).

(c) Para la frecuencia: se especifica la intensidad del continuo en el borde de alta frecuencia del perfil de línea.

Para resolver el sistema de ecuaciones 5.42, se deben discretizar las ecuaciones en una grilla de valores de p, z y $\nu$. Un esquema de eliminación para la resolución puede verse en los trabajos Mihalas et al. (1975) y Mihalas et al. (1976). 


\subsection{Códigos de cálculo para vientos impulsados por radiación}

En base a las ideas desarrolladas en la teoría de vientos impulsados por radiación, y siguiendo los diferentes lineamientos planteados en la sección 2.2.9, se desarrollaron varios códigos de cálculo de atmósfera que resuelven el transporte de radiación tal como se plantea en las secciones 5.1.1, 5.1.2 y 5.1.3. Estos códigos se usan extensamente en la actualidad.

En la tabla 5.1, adaptada de Puls (2008), se resumen las características de estos códigos y sus aplicaciones.

\begin{tabular}{|c|c|c|c|c|c|c|c|}
\hline & $\begin{array}{l}\text { Detail/ } \\
\text { Surface }\end{array}$ & TLUSTY & CMFGEN & WM-basic & FASTWIND & PoWR & PHOENIX \\
\hline Autor & Butler & Hubeny & Hillier & Pauldrach & Puls & Hamann & Hauschildt \\
\hline Geometría & $\begin{array}{l}\text { plano- } \\
\text { paralela }\end{array}$ & $\begin{array}{l}\text { plano- } \\
\text { paralela }\end{array}$ & esférica & esférica & esférica & esférica & esférica \\
\hline Blanketing & LTE & si & si & si & aproximado & si & si \\
\hline $\begin{array}{l}\text { Transporte } \\
\text { en líneas }\end{array}$ & $\begin{array}{l}\text { marco del } \\
\text { observador }\end{array}$ & $\begin{array}{l}\text { marco del } \\
\text { observador }\end{array}$ & $\begin{array}{l}\text { comoving } \\
\text { frame (CMF) }\end{array}$ & Sobolev & $\mathrm{CMF}$ & $\mathrm{CMF}$ & $\begin{array}{l}\text { CMF - } \\
\text { observador }\end{array}$ \\
\hline $\begin{array}{l}\text { Estructura de } \\
\text { temperatura }\end{array}$ & $\begin{array}{l}\text { equilibrio } \\
\text { radiativo }\end{array}$ & $\begin{array}{l}\text { equilibrio } \\
\text { radiativo }\end{array}$ & $\begin{array}{l}\text { equilibrio } \\
\text { radiativo }\end{array}$ & $\begin{array}{l}\text { balance } \\
\text { térmico } \mathrm{e}^{-}\end{array}$ & $\begin{array}{l}\text { balance } \\
\text { térmico } \mathrm{e}^{-}\end{array}$ & $\begin{array}{l}\text { equilibrio } \\
\text { radiativo }\end{array}$ & $\begin{array}{l}\text { equilibrio } \\
\text { radiativo }\end{array}$ \\
\hline fotosfera & si & si & de TLUSTY & aproximada & si & si & si \\
\hline $\begin{array}{l}\text { Región de } \\
\text { diagnóstico }\end{array}$ & $\begin{array}{l}\text { sin } \\
\text { límites }\end{array}$ & $\begin{array}{l}\text { sin } \\
\text { límites }\end{array}$ & $\begin{array}{l}\text { sin } \\
\text { límites }\end{array}$ & UV & $\begin{array}{l}\text { óptico - } \\
\text { IR }\end{array}$ & $\begin{array}{l}\text { sin } \\
\text { límites }\end{array}$ & $\begin{array}{l}\text { sin } \\
\text { límites }\end{array}$ \\
\hline $\begin{array}{l}\text { Aplicación } \\
\text { principal }\end{array}$ & $\begin{array}{l}\text { estrellas } \\
\text { calientes con } \\
\text { viento } \\
\text { despreciable }\end{array}$ & $\begin{array}{l}\text { estrellas } \\
\text { calientes con } \\
\text { viento } \\
\text { despreciable }\end{array}$ & $\begin{array}{l}\text { estrellas } \\
\mathrm{OB}(\mathrm{A}) \text { - } \\
\mathrm{WR}- \\
\mathrm{SNe}\end{array}$ & $\begin{array}{l}\text { estrellas } \\
\text { con vientos } \\
\text { densos - } \\
\text { SNe }\end{array}$ & $\begin{array}{l}\text { estrellas } \\
\text { OB - } \\
\text { A sgs } \\
\text { tempranas }\end{array}$ & WR & $\begin{array}{l}\text { estrellas } \\
\text { debajo de } \\
\text { 10kK - } \\
\text { SNe }\end{array}$ \\
\hline Comentarios & Sin viento & Sin viento & $\begin{array}{l}\text { Requiere } \\
\text { modelo de } \\
\text { fotosfera }\end{array}$ & $\begin{array}{l}\text { sin } \\
\text { clumping }\end{array}$ & $\begin{array}{l}\text { elementos } \\
\text { explícitos } \\
\text { - background }\end{array}$ & & $\begin{array}{l}\text { incluye } \\
\text { moléculas } \\
\text { sin clump. }\end{array}$ \\
\hline $\begin{array}{l}\text { Tiempo de } \\
\text { ejecución }\end{array}$ & $\begin{array}{l}\text { pocos } \\
\text { minutos }\end{array}$ & horas & horas & 1 a 2 horas & $\begin{array}{l}\text { pocos minutos } \\
\text { a media hora }\end{array}$ & horas & horas \\
\hline
\end{tabular}

Tabla 5.1. Características básicas de los códigos de atmósfera actuales. Adaptado de Puls (2008)

Las referencias principales para cada código y algunas de sus aplicaciones se enumeran a continuación:

- Detail/Surface: Butler \& Giddings (1985).

- TLUSTY: Hubeny (1988), Hubeny \& Lanz (1992), Hubeny \& Lanz (1995), (Lanz \& Hubeny 2003).

- CMFGEN: Hillier (1987), Hillier \& Miller (1998), Hillier \& Lanz (2001), Hillier (2012).

- WM-basic: Pauldrach et al. (2001), Sternberg et al. (2003).

- FASTWIND: Santolaya-Rey et al. (1997), Puls et al. (2005), Rivero González et al. (2012).

- PoWR: Hamann \& Schmutz (1987), Todt et al. (2015).

- PHOENIX: Hauschildt \& Baron (1999), Jack et al. (2009). 


\subsubsection{El código FASTWIND}

FASTWIND es un código para la resolución de atmósferas en NLTE, diseñado para el análisis espectroscópico de estrellas tempranas y luminosas $\mathrm{O}, \mathrm{B}$ y A con vientos estelares (aunque también se lo ha usado para al análisis de estrellas centrales de nebulosas planetarias). El código ha sido desarrollado y, a su vez, es mantenido y actualizado por el Dr. J. Puls y colaboradores, pertenecientes a la Universidad de Munich, Alemania. La presentación de FASTWIND (aún sin la denominación actual) fue hecha en el trabajo de Santolaya-Rey et al. (1997), donde se reunen las principales características del código.

FASTWIND ha sido construido para obtener la radiación emergente de un sistema hidrodinámico dado por el usuario, razón por la cual, sigue los lineamientos del inciso 3 de la sección 2.2.9. Con las modificaciones realizadas para esta tesis, el código se adecua a los lineamientos del inciso 2 de esa sección.

La intención de los programadores de FASTWIND (y de ahí, la razón del nombre del código) consiste en construir un extenso programa capaz de resolver los cálculos necesarios de una manera ágil, en tiempos cortos de computación, haciendo uso de aproximaciones que eviten los extensos ciclos de iteración pero que conserven un buen nivel de precisión, en comparación con otros códigos más rigurosos. En su concepción, otros requisitos fueron también la robustez del código, capaz de procesar modelos de estrellas bajo condiciones físicas muy diferentes, y la portabilidad, siendo sencillo de instalar y reducido en sus requerimientos de hardware. Su objetivo es analizar muestras grandes de espectros de estrellas masivas en un tiempo de cálculo razonable, incorporando progresivamente, nuevos procesos físicos de alta complejidad.

Este código se aplica a sistemas estacionarios, con simetría esférica, haciendo uso del concepto de atmósferas unificadas (sección 2.3). De este modo, en FASTWIND, se construye una fotósfera en pseudo-equilibrio hidrostático, con geometría esférica y con la extensión espacial necesaria. La fotosfera es obtenida a partir de modelos planoparalelos corregidos en extensión y esfericidad, para evitar cálculos que incrementarían considerablemente los tiempos de ejecución del programa. Los parámetros fundamentales de la estrella en consideración ( $\mathrm{T}_{\mathrm{eff}}, \log \mathrm{g}, \mathrm{R}_{*}$, abundancias) son brindados como datos de entrada por el usuario.

La fotosfera se empalma a un viento estelar en una región de transición (la región del punto sónico) de baja velocidad. Los parámetros del viento: $\mathrm{v}_{\infty}, \dot{M} \mathrm{y} \beta$, son los correspondientes a una ley $\beta$ de velocidades fija (ecuación 2.2 , cuyos valores también deben ser suministrados de antemano. La estructura conjunta formadas por la fotosfera y el viento permiten obtener, de manera consistente, con el mismo grado de precisión y bajo las mismas condiciones de cálculo, los perfiles de línea sintéticos originados en cualquiera de estas regiones, o los perfiles que resulten de la combinación de aportes de cada parte.

La estructura de temperatura en la fotosfera y la aceleración del continuo de radiación también son aproximadas para emular la condición de equilibrio radiativo (evitando el proceso iterativo correspondiente que demandaría un extenso tiempo de cómputo). En la región externa del viento, la temperatura es mantenida constante a un valor mínimo, $\mathrm{T}_{\text {min }}=0.75 \mathrm{~T}_{\text {eff }}$, que es característico de los modelos plano-paralelos NLTE para tipos espectrales comprendidos entre $\mathrm{O}$ y A. Una vez construido el modelo hidrodinámico, el código calcula las opacidades usando una base de datos atómicos dispuestos 
en un formato común y dinámico (data driven input), que permite cambiar las características de las especies atómicas involucradas (niveles de energía, secciones eficaces, intensidades de oscilador, etc.), sin requerir la modificación del código.

FASTWIND resuelve el transporte de radiación en medios en movimiento para la fotosfera y el viento. Las primeras 10 iteraciones de estas operaciones se calculan con radiación del continuo solamente, sin tener en cuenta las líneas espectrales. Luego siguen 10 iteraciones más, bajo la aproximación de Sobolev con continuo (sección 5.1.2). Finalmente, en la iteración 20, el programa puede pasarse al cálculo riguroso en el CMF. Por lo tanto, es optativo la convergencia final tanto en la aproximación de Sobolev como en el CMF.

Con estos desarrollos, es posible obtener el continuo de radiación y los perfiles de líneas espectrales de varios elementos, básicamente para el $\mathrm{H}$, el He I y el He II, en el rango de longitudes del óptico y en el IR. También pueden obtenerse líneas espectrales para el Si II, Si III, Si IV, N II, N III, N IV y NV. Las poblaciones de los iones mencionados son calculados de forma explícita, con toda la precisión, pero el programa también calcula de manera aproximada las poblaciones de los niveles de una numerosa tabla de iones.

Una versión actualizada de FASTWIND fue presentada en el trabajo de Puls, Urbaneja, Venero, et al. (2005). Una parte de las mejoras sobre el código incluidas en ese trabajo, es presentada en las secciones siguientes, dando inicio al desarrollo de los aportes propios del autor a la presente tesis. Específicamente, se trata de la incorporación al código del tratamiento de las inversiones de poblaciones atómicas, efecto que genera importantes problemas de convergencia en la resolución del transporte de radiación, tanto en la aproximación de Sobolev con continuo, como en el comoving frame. Estos temas son desarrollados en las secciones 5.3.1 y 5.3.2.

En la nueva versión se incluyó el fenómeno de line-blocking/blanketing (sección 2.3), resolviendo las ecuaciones de equilibrio estadístico de una manera aproximada con miras a reducir los tiempos de cómputo. Para incorporar este fenómeno se usaron opacidades y emisividades medias en las líneas en una aproximación estadística.

También, en ese trabajo, se presentó una detallada comparación de resultados generados con FASTWIND, con los obtenidos por otros dos códigos: CMFGEN y WM-Basic (sus características se presentan en la sección 5.2). Los tres códigos predicen las mismas estructuras de temperatura y flujos radiativos para $\lambda>400 \AA$. Las diferencias más significativas aparecen en el continuo del He II, es decir, para longitudes de onda menores a la indicada, debido a la sensibilidad de los flujos ionizantes a pequeñas diferencias entre los modelos empleados en los diferentes códigos. La comparación de los perfiles de línea también muestra un alto nivel de coincidencia, exceptuando algunas líneas de singuletes de He I, en regímenes de alta temperatura.

La última actualización del código fue presentada en el trabajo de Rivero González et al. (2012), con la incorporación de un nuevo modelo atómico para el elemento N. Los perfiles derivados fueron usados para determinar, con mucha precisión, las abundancias de este elemento en estrellas $\mathrm{O}$, con objeto de probar las predicciones de los cálculos evolutivos. 


\subsection{El problema de las inversiones de población}

El análisis del problema de las inversiones de población, su aplicación y testeo en el código FASTWIND (hasta la sección 2.5), fue realizado por el autor (a menos que se lo indique expresamente) y forma parte del trabajo de esta Tesis.

En el modelado de las regiones externas de las atmósferas de las estrellas tempranas pueden producirse inversiones en las poblaciones de los niveles atómicos. Una inversión consiste en que, para una dada transición, el nivel de mayor energía $(u)$ se encuentra sobrepoblado respecto a la población del nivel inferior $(l)$. En esas circunstancias, en el coeficiente de opacidad de la línea dado por la expresión:

$$
\kappa_{L}=\frac{\pi e^{2}}{m_{e} c} \frac{f_{u l} g_{u}}{\rho}\left[\frac{n_{l}}{g_{l}}-\frac{n_{u}}{g_{u}}\right],
$$

se produce la configuración $n_{u} / g_{u}>n_{l} / g_{l}$, lo que genera opacidades negativas y, por ende, profundidades ópticas también negativas. Habitualmente, las inversiones de población son pequeñas, pero aún así, pueden complicar la convergencia en el tratamiento de las ecuaciones acopladas de transporte de radiación y de equilibrio estadístico.

Los problemas que generan las inversiones se resumen a continuación:

- Las inversiones suelen aparecer generando una población atómica oscilante, con leves inversiones que se alternan con configuraciones electrónicas normales. Esta secuencia de inversión y no inversión, conduce a problemas en la convergencia de las ecuaciones de equilibrio estadístico.

- Las inversiones de población pueden producir que, en la región de transición entre una población "normal" y una sobrepoblada, la función fuente de línea diverja, especialmente cuando se están calculando líneas espectrales en el IR. A modo de ejemplo simplificado, puede considerarse la expresión para una función fuente en un átomo de dos niveles:

$$
S_{u l}=\frac{n_{u} A_{u l}}{n_{l} B_{l u}-n_{u} B_{u l}}=\frac{2 h \nu^{3}}{c^{2}} \frac{1}{\left(\frac{n_{l} g_{u}}{n_{u} g_{l}}-1\right)}
$$

donde $A_{u l}, B_{u l}$ y $B_{l u}$ son los coeficientes de Einstein de probabilidad de emisión espontánea e inducida, y de absorción, respectivamente. La última igualdad se obtiene a partir de las relaciones de Einstein:

$$
\left\{\begin{array}{l}
A_{u l}=\frac{2 h \nu^{3}}{c^{2}} B_{u l} \\
g_{l} B_{l u}=g_{u} B_{u l}
\end{array}\right.
$$

Expresando los cocientes de poblaciones por medio de un coeficiente de apartamiento de equilibrio termodinámico local (ETL), por ejemplo $b_{l}=n_{l} / n_{l}^{*}$, donde las poblaciones indicadas con asterisco corresponden a las poblaciones en ETL, puede escribirse: 


$$
\frac{n_{l} g_{u}}{n_{u} g_{l}}=\frac{b_{l} n_{l}^{*} g_{u}}{b_{u} n_{u}^{*} g_{l}}=\frac{b_{l}}{b_{u}} e^{h \nu / k T},
$$

en la que se usó la ley de Boltzmann para las poblaciones en ETL. Considerando que $b_{u}=b_{l}+\epsilon$, donde $\epsilon$ representa la sobrepoblación del nivel durante una inversión, entonces:

$$
\frac{n_{l} g_{u}}{n_{u} g_{l}}=\frac{b_{l}}{b_{l}+\epsilon} e^{h \nu / k T}=\frac{e^{h \nu / k T}}{1+\epsilon / b_{l}} \approx \frac{1}{1+\epsilon / b_{l}},
$$

donde, en la última relación, se aplica que $h \nu / k T \ll 1$, para el IR lejano o intermedio.

En el caso de una inversión, el cociente de la ecuación 5.50 es cercano o menor a 1, por lo cual la función fuente de línea (ecuación 5.47), puede diverger o hacerse negativa.

- En el caso de fuertes inversiones la intensidad media $\bar{J}$ (integrada en frecuencia) dada por la ecuación 5.35, puede volverse muy pequeña e, incluso, tomar valores negativos. Eso sucede porque la probabilidad de escape $\beta_{s}$ (ecuación 5.36) crece fuertemente debido a que la profundidad óptica se hace negativa $(\tau<0)$, y en esa expresión, aparece con dependencia $e^{-\tau}$. Entonces, el término negativo de la ecuación 5.35 domina esta ecuación, generando una intensidad media que desestabiliza la convergencia.

En otros códigos de cálculo, el problema de las inversiones es "resuelto" o, expresado más adecuadamente, evitado, forzando una condición de LTE en la población del nivel superior de la transición con respecto al más bajo, o con otras aproximaciones semejantes. Sin embargo, para obtener una solución apropiada a este problema, es necesario reformular de un modo correcto la ecuación de transporte y tratar, con especial detalle, la aproximación de Sobolev con continuo.

El primer tratamiento teórico del problema de las inversiones poblacionales, para el caso de la aproximación de Sobolev en el continuo, fue propuesto por Taresch et al. (1997), aunque su implementación en el código FASTWIND fue solamente parcial, atendiendo a los casos más sencillos de inversión.

En esta parte del trabajo de tesis se amplia el tratamiento de las inversiones propuesto por Taresch et al. (1997), para cubrir un mayor escenario de condiciones de inversión en la resolución de transporte por medio de la aproximación de Sobolev con continuo. Luego se implementa una resolución alternativa para este problema para el caso del transporte de radiación en el CMF. Finalmente, se aplican completamente estos métodos al código FASTWIND y se realiza un extenso testeo de su funcionamiento.

\subsubsection{Inversiones de población en la aproximación de Sobolev con continuo}

En la sección 5.1.2 se describe la metodología necesaria para la resolución del transporte en la aproximación de Sobolev con continuo. La solución obtenida es la ecuación 
5.35 que puede considerarse como una generalización de la solución en la que sólo se tienen en cuenta los procesos de línea, la que sólo contiene los dos primeros términos de esa ecuación. Para obtener la solución completa, es necesario evaluar la función de interacción $U(\tau, \beta)$, que se define por la ecuación 5.34 y se transcribe a continuación:

$$
\begin{aligned}
U\left(\tau, \frac{\kappa_{C}}{\kappa_{L}^{\prime}}\right) \equiv & \tau \int_{-\infty}^{\infty} d \xi \phi(\xi) \int_{-\infty}^{\xi} d \xi^{\prime} \phi\left(\xi^{\prime}\right) \\
& \times \exp \left[-\tau \int_{\xi}^{\xi^{\prime}} d s \phi(s)\right]\left\{1-\exp \left[-\frac{\kappa_{C}}{\kappa_{L}^{\prime}} \tau\left(\xi^{\prime}-\xi\right)\right]\right\},
\end{aligned}
$$

En esta ecuación se ha seguido la notación de Taresch et al. (1997), especialmente, se usa $\kappa_{L}^{\prime}=\kappa_{L} / \Delta \nu_{D}$, que es equivalente a la función $\kappa_{L}$ de Hummer \& Rybicki (1985), de modo que $\beta=\kappa_{C} / \kappa_{L}^{\prime}$.

Es necesario notar que, para evaluar $\bar{J}$, según la ecuación 5.35 , es necesario integrar la función $U\left(\tau, \kappa_{C} / \kappa_{L}^{\prime}\right)$ en los ángulos sólidos, expresión que, seguidamente, se denomina como $\bar{U}$ :

$$
\bar{U}=\int d \omega U(\tau, \beta),
$$

De esta manera, la expresión de $\bar{J}$ para la ecuación 5.35 queda de una forma semejante a la encontrada en la aproximación de Sobolev pura (sin continuo), ecuación 5.17, de:

$$
\bar{J}=\left(\overline{\beta_{c} I_{c}}+\bar{U} S_{C}\right)+(1-\beta-\bar{U}) S_{L}
$$

La programación en el código FASTWIND de esa función se lleva a cabo mediante la interpolación en una tabla extensa de valores de $U(\tau, \beta)$, calculados para una fina grilla de parámetros $\tau$ y $\beta$. De este modo, se evita el cálculo directo de la función para cada punto $\tau$ a lo largo del viento; lo cual consume mucho tiempo de cómputo y reduce la eficiencia y rapidez del código.

Puls \& Hummer (1988) tabularon una parte de la función $U(\tau, \beta)$, para un perfil Doppler $\phi(\xi)$, con valores $\tau>0$ y $\beta=\kappa_{C} / \kappa_{L}^{\prime} \leq 0.01$, siendo extendido al intervalo $10^{-10} \leq \beta \leq 10^{5}$ por Taresch en su Tesis Doctoral (1992).

Precisamente, la función $\overline{U(\tau, \beta)}$ es una función clave en los problemas de convergencia que surgen para niveles invertidos, ya que las integrales que la componen crecen exponencialmente en esas circunstancias, especialmente si el continuo es dominante $(\beta$ $>1$ y $\tau<0)$.

Para evitar estos problemas numéricos, Taresch et al. (1997) propone reescribir $\bar{U}$ como:

$$
\bar{U}=U_{1}+U_{2}, \quad U_{1}=1-\beta, \quad U_{2}=\bar{U}-1+\beta,
$$

\footnotetext{
${ }^{2}$ Notar que, a diferencia del resto de la nomenclatura, en este caso la barra superior no indica integración sobre las frecuencias
} 
donde

$$
\begin{aligned}
U_{2}= & |\tau| \int_{-\infty}^{\infty} d \xi \phi(\xi) \int_{\xi}^{\infty} d \xi^{\prime} \phi\left(\xi^{\prime}\right) \\
& \times \exp \left(|\tau| \int_{\xi}^{\xi^{\prime}} d s \phi(s)-\left|\frac{\kappa_{C}}{\kappa_{L}^{\prime}} \tau\right|\left(\xi^{\prime}-\xi\right)\right),
\end{aligned}
$$

que tiene la ventaja de tender a 0 cuando el continuo es dominante, es decir cuando $\kappa_{C} / \kappa_{L}^{\prime} \rightarrow \infty$. De lo contrario, si la línea es dominante $\left(\kappa_{C} \ll \kappa_{L}^{\prime}\right)$, esta función puede ser aproximada como $-(1-\beta)$, por lo cual $\bar{U}$ tiende a 0 , recuperándose la aproximación de Sobolev estándar.

La implementación completa de estos desarrollos en el código FASTWIND fueron presentados por Puls, Urbaneja, Venero, et al. (2005) y es expuesta en la sección 5.3.3

\subsubsection{Inversiones de población en el comoving frame}

Puls, Urbaneja, Venero, et al. (2005) presentaron, por primera vez, el tratamiento del problema de las inversiones de población en el CMF. En este marco de referencia para la resolución de la ecuación de transporte, la divergencia de la función fuente es inevitable para el formalismo estándar presentado en la sección 5.1.3.

Aún cuando las cantidades locales no diverjan, siempre habrá una divergencia implícita entre dos puntos de profundidad sucesivos, antes y al comienzo de una región con sobrepoblación, lo cual, debido al proceso de discretización de las ecuaciones, no podrá desarrollarse de manera consistente.

Para evitar este problema, resulta más adecuado trabajar directamente con emisividades y opacidades, en lugar de definir una función fuente. También, es apropiado reescribir las ecuaciones usando directamente la coordenada z, en lugar de definir las profundidades ópticas.

Por lo tanto, en el caso de inversiones, se resuelven las dos ecuaciones acopladas de transporte radiativo en el CMF, equivalentes a las ecuaciones 5.42 , con el sistema:

$$
\begin{aligned}
& \frac{\partial U}{\partial z}-\frac{\partial V}{\partial x}=-\chi V \\
& \frac{\partial V}{\partial z}-\frac{\partial U}{\partial x}=\eta-\chi U,
\end{aligned}
$$

donde U y V son las variables usuales de Feautrier (ecuaciones 5.41), $x$ es la frecuencia medida desde el centro de la línea en unidades de ancho Doppler (ecuación 2.30 y $z$ es la variable de profundidad a lo largo del parámetro de impacto. La opacidad es $\chi=\chi_{C}(z)+\chi_{L}(z, x)$ y la emisividad, $\eta=\eta(z, x)$.

\subsubsection{Implementación en FASTWIND del tratamiento de las inversiones de población}

Para incorporar la corrección por las inversiones poblacionales en la aproximación de Sobolev con continuo en el código FASTWIND, es necesario construir una tabla adecuada para los valores de la función $U_{2}$, dada por la ecuación 5.55. Esta función 
tiene el aspecto dado por la figura 5.2 , que ilustra a la misma en función de $\log \beta$, para diferentes valores de $\tau$. Se trata de una función complicada, que exhibe una dependencia diferente para distintos rangos de $\beta$ y de $\tau$.

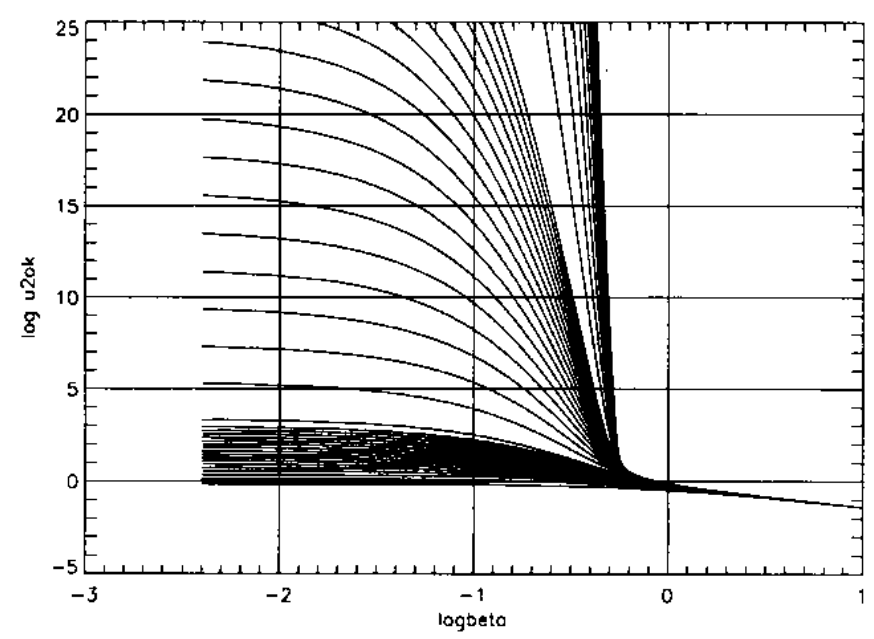

Figura 5.2. Diagrama logarítmico de $U_{2}$ en función de $\log \beta$, cada curva corresponde a un dado valor de $\tau$.

Por este motivo, una única tabla para $U_{2}$ no permite obtener la precisión adecuada para los diferentes valores de las variables de las que depende. Se hace necesario muestrear diferencialmente los valores de $U_{2}$, en regiones separadas del plano $(\beta, \tau)$, tal como se presenta en la figura 5.3(a). Las características de cada una de estas regiones pueden encontrarse en la tabla 5.2. Las aproximaciones de la periferia han sido encontradas mediante desarrollos a primer orden de las exponenciales o usando los límites apropiados. La tabla ha sido ampliada y reajustada en comparación a la calculada por Taresch et al. (1997).

Para la implementación en el CMF, se discretizaron las ecuaciones 5.56 con respecto a $z$ y a $x$. Se adoptó un esquema fully implicit (Mihalas et al. 1975, Apéndice B). De acuerdo al trabajo de Mihalas, este método es incondicionalmente estable.

Se llevaron a cabo numerosas pruebas del funcionamiento del código FASTWIND, incluyendo el tratamiento de las inversiones tanto en la aproximación de Sobolev con continuo, como en el CMF. A modo ilustrativo, la figura 5.3(b) muestra la localización en el plano $(\beta, \tau)$ de los puntos requeridos a las tablas de $U_{2}$ por FASTWIND durante una ejecución de prueba. Cada uno de esos puntos habría producido inversiones que, sin el tratamiento adecuado a través del uso de las tablas para $U_{2}$, habrían sido calculados de manera muy aproximada o, directamente, excluidas (por ejemplo, ajustando a cero la función fuente de línea en cada caso).

Cabe destacar que el formato de la nueva discretización de las ecuaciones de transporte en el CMF fue puesto a prueba con modelos que no registraban inversiones, efectuando el cálculo de todas las transiciones en base a las opacidades y emisividades, y en la coordenada $z$, en lugar de las profundidades ópticas y la función fuente. Todas las pruebas dieron resultados satisfactorios, con un alto grado de acuerdo entre la 


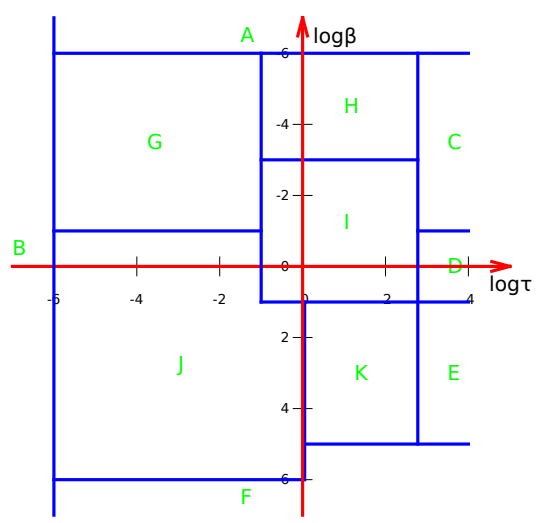

(a)

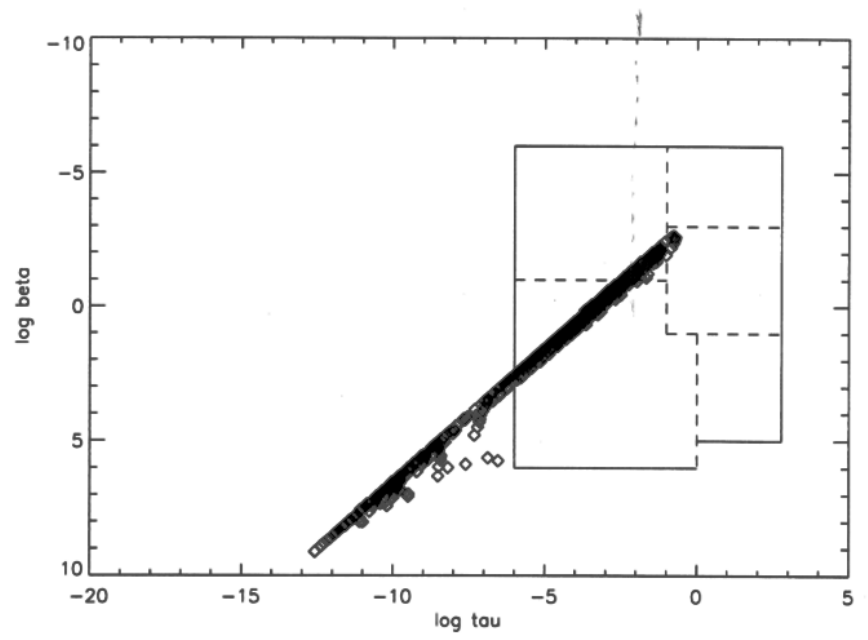

(b)

Figura 5.3. a) Regiones de diferente grillado o de aproximaciones límite (referencias en la tabla 5.2), usadas para construir un mapa de valores para interpolar la función $U_{2}$ en función de $\log \beta \mathrm{y} \log \tau$. Los valores de la periferia son, mayormente, aproximaciones analíticas. b) Localización de valores de la tabla de $U_{2}$ requeridos durante una de las ejecuciones de prueba de FASTWIND, realizada para determinar la precisión del grillado de cada región.

\begin{tabular}{|c|c|}
\hline Áreas & Grillado o aproximación para $U_{2}$ \\
\hline A & $U_{2} \approx\left(e^{\tau}-1\right) / \tau-1=\beta-1$ \\
\hline $\mathrm{B}$ & $\overline{U_{2}} \approx \tau / 2$ \\
\hline $\bar{C}$ & Similar a A \\
\hline $\mathrm{D}^{-}$ & Extrapolación de tabla I \\
\hline$\overline{\mathrm{E}}$ & Extrapolación de täbla $\overline{\mathrm{K}}$ \\
\hline $\mathrm{F}$ & $U_{2} \approx 1 /(\sqrt{2 \pi \beta}$ \\
\hline$\overline{\mathrm{G}}$ & $\overline{U_{2}} \approx \tau / 2$ (interpola $\left.\overline{U_{2}} / \bar{\tau}\right)$ \\
\hline$\overline{\mathrm{H}}$ & Similar a $\mathrm{A}$ \\
\hline $\mathrm{I}^{-}$ & Grilla do fino $(\overline{1} \overline{9} 2 \times \overline{2} 00)$ \\
\hline $\mathrm{J}$ & Grillado $(6 \overline{1} \times$ \\
\hline & Grillàdo grueso (12 x 18$)$ \\
\hline
\end{tabular}

Tabla 5.2. Sectorización de la tabla para la función $U_{2}$, dependiente de los valores de $\beta$ y $\tau$, presentadas en la figura $5.3(\mathrm{a})$.

implementación nueva y la estándar.

Después de un exhaustivo seguimiento de la ejecución del código, se procedió a una comparación detallada entre modelos calculados con versiones previas y los mismos, obtenidos con la versión mejorada. Se probó para un grilla de estrellas O, con resultados muy satisfactorios, verificando la completa ausencia de los problemas de convergencia originados en el tratamiento anterior al propuesto en este trabajo. 
Las diferencias en los perfiles de línea del $\mathrm{H}$ y del He (tanto en el óptico como en el IR) son muy pequeñas, lo que muestra el potencial del método aplicado. Sin embargo, es necesario aclarar que el tratamiento de las inversiones de población ha sido implementado solamente en el viento. En el caso de estrellas con pérdida de masa extrema y un número muy abundante de transiciones de metales en el IR, las inversiones pueden ocurrir en la fotosfera de la estrella, por lo cual, un nuevo tratamiento de estos casos sería requerido. 


\section{Capítulo 6}

\section{Análisis de los perfiles de línea para las diferentes soluciones hidrodinámicas}

Resumen del capítulo: Mediante el código de atmósfera NLTE FASTWIND, se resuelve la ecuación de transporte de radiación para medios en movimiento, y se calculan los perfiles de líneas espectrales emergentes para las soluciones hidrodinámicas de tipo rápida, $\Omega_{\text {lenta }} y \delta_{\text {lenta }}$. Se evalúa la influencia que ejercen los parámetros de la estrella, del viento $y$ de la fuerza de radiación $(k, \alpha$ y $\delta$ ) sobre la intensidad y la morfología

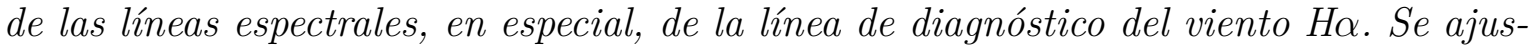
tan los perfiles sintéticos de las líneas espectrales a observaciones espectroscópicas de algunas estrellas supergigantes $B$, para evaluar la factibilidad de las soluciones para modelar el viento de estas estrellas.

\subsection{Modelado de perfiles de líneas espectrales}

En los capítulos previos se obtuvieron soluciones numéricas a partir de las ecuaciones hidrodinámicas que describen a un viento rotante impulsado por radiación, en torno a una estrella supergigante B. Las soluciones comprenden dos regímenes de vientos bien diferenciados: el régimen rápido y el régimen lento. Además, los parámetros globales de los vientos obtenidos en estas soluciones, tales como $\dot{M} \mathrm{y} \mathrm{v}_{\infty}$, son capaces de generar valores acordes a los estimados para esta clase de estrellas.

Sin embargo, para avanzar en una evaluación más detallada de la capacidad de estas soluciones para representar las condiciones físicas de los vientos estelares, es necesario comparar directamente características observables. Una alternativa sería modelar, a partir de las soluciones del viento, los excesos en longitudes de onda del IR o radio. Sin embargo, una descripción más minuciosa de los vientos sólo puede hacerse a partir del modelado de los perfiles de línea. Esta metodología es más trabajosa y requiere de un código suficientemente sofisticado como para resolver la ecuación de transporte en medios en movimiento para los entornos físicos de los vientos estelares. Sin embargo, el modelado de las líneas ofrece un potencial mayor debido a que, en los perfiles, hay mucha más información acerca de la forma del campo de velocidades y de la distribución 
de densidades a lo largo del viento.

En este trabajo se opta por modelar las líneas espectrales y para ello se usa el código FASTWIND, ya presentado en la sección 5.2.1. Los iones cuyas líneas son las más relevantes para evaluar la estructura de los vientos corresponden a los elementos más abundantes en los vientos: el hidrógeno y el helio. Las líneas de este último elemento pueden pertenecer a cada uno de sus dos primeros estados de ionización, He I y He II, aunque las líneas de He II sólo son apreciables en los espectros de las estrellas más calientes.

Particularmente importantes son las líneas de la serie de Balmer del H I, en especial, la línea $\mathrm{H} \alpha$ cuya estructura está fuertemente determinada por el viento, línea que se estudia con mayor detalle en esta tesis.

La lista de transiciones calculadas con FASTWIND para los modelos de este trabajo se detallan en la tabla 6.1, a la cual se agregan líneas de iones de importancia secundaria, como es el caso de Si II, III y IV. Las líneas de Si que pertenecen al rango visible del espectro se usan, habitualmente, para diagnosticar las temperaturas efectivas a lo largo de los diferentes subtipos espectrales (sección 1.2.3). Las líneas del Si IV, ubicadas en el UV, son empleadas para la medición de las velocidades terminales (sección 1.2.6). Ambos análisis no se llevan a cabo en este trabajo ya que, en el primer caso, no se pretenden explorar las condiciones físicas de la fotosfera y, en el segundo caso, el código de cálculo FASTWIND no está optimizado para resolver el transporte de radiación en el rango espectral UV.

\begin{tabular}{|c|lll|}
\hline Ion & \multicolumn{3}{|c|}{$\boldsymbol{\lambda}[\AA]$} \\
\hline \hline H I & $\mathrm{H} \alpha$ & 6563 & \\
& $\mathrm{H} \beta$ : & 4861 & \\
& $\mathrm{H} \gamma:$ & 4341 & \\
& $\mathrm{H} \delta:$ & 4182 & \\
& $\mathrm{H} \epsilon$ : & 3970 & \\
\hline He I & 4026 & 4387 & 4471 \\
& 4713 & 4922 & 6678 \\
\hline He II & 4200 & 4541 & 4686 \\
& 6406 & 6527 & 6683 \\
\hline Si II & 4128 & 4130 & 5041 \\
& 5056 & & \\
\hline Si III & 4552 & 4567 & 4574 \\
& 4716 & 4813 & 4819 \\
& 4829 & 5739 & \\
\hline Si IV & 4089 & 4116 & 4212 \\
& 4950 & 6667 & 6701 \\
\hline
\end{tabular}

Tabla 6.1. Sumario de las líneas espectrales calculadas con la versión disponible de FASTWIND. 


\subsection{Vinculación entre los códigos HYDWIND y FASTWIND}

Como se detalló en la sección 3.3.5, por medio del código HYDWIND, es posible obtener las soluciones hidrodinámicas del viento para estrellas supergigantes B en rotación, siguiendo los lineamientos de los trabajos de Curé (2004); Curé et al. (2011). Para el cálculo de los perfiles sintéticos de líneas se usa el código de atmósfera NLTE FASTWIND (sección 5.2.1).

El código FASTWIND no resuelve las ecuaciones de movimiento ya que, para dar la estructura hidrodinámica del viento, utiliza originalmente una ley $\beta$ de velocidades (ecuación 2.2), cuyos parámetros $\mathrm{v}_{\infty}$ y $\dot{M}$ deben ser provistos de antemano.

Esta restricción dada por la adopción de un campo de velocidades fijo, no permite elaborar un análisis directo sobre la influencia de la fuerza de radiación en el campo de velocidades y en la distribución de densidades resultante (consistente con la ecuación de continuidad, ecuación 2.3). Es necesario, entonces, reemplazar en el código FASTWIND la ley $\beta$ de velocidades por las soluciones hidrodinámicas numéricas calculadas con HYDWIND (descriptas en la sección 3.3.5).

Esta modificación ha sido realizada por el Dr. J. Puls, y ha sido testeada extensamente por el autor de esta tesis, habilitando las potencialidades de FASTWIND para la resolución del problema del transporte de radiación en un viento con las características propias de las soluciones de Curé.

A partir de la versión modificada del código FASTWIND, se organiza una rutina de cálculo de perfiles sintéticos, que consiste en la siguiente secuencia:

1) Construcción de un archivo de entrada de datos, con los valores de los parámetros fundamentales de la estrella: temperatura efectiva, gravedad superficial, radio, velocidad de rotación, abundancias y parámetros de la fuerza de radiación.

2) Obtención de una solución hidrodinámica que verifique las condiciones de continuidad de la velocidad y de su gradiente, a partir del código HYDWIND. Estas soluciones brindan los valores globales que definen al viento estelar: su velocidad terminal $\mathrm{v}_{\infty}$, y su tasa de pérdida de masa $\dot{M}$, junto con una tabla de valores para el campo de velocidades, sus gradientes, la ley de densidad y otras magnitudes relevantes.

3) Ingreso de estos datos en el código FASTWIND, y por su intermedio, el cálculo de una fotosfera y la adición al viento de la ley de temperaturas y los números ocupacionales afines al equilibrio de ionización y de excitación. A partir de estos valores, la obtención del continuo de radiación y de los perfiles de línea buscados, como solución de la ecuación de transporte en la aproximación de Sobolev (sección 5.1.2) y en el "comoving-frame" (sección 5.1.3). Estos cálculos hacen uso del tratamiento de las inversiones de población (sección 5.3.3). 


\subsection{Análisis de los perfiles y los parámetros de la fuerza de radiación}

En el capítulo 4 se investigó la influencia de los parámetros de la fuerza de radiación $k, \alpha$ y $\delta$, junto con la tasa rotacional $\Omega$, sobre las soluciones de los vientos para los regímenes rápido y lento. También se estudiaron las diferencias en los dominios de las soluciones encontradas para los subtipos espectrales correspondientes a las supergigantes B. A continuación se analiza cómo se manifiestan estas relaciones en el aspecto de los perfiles de línea.

\subsubsection{Dependencia de los perfiles de línea con $\Omega$ y $\delta$}

El modelo base T19 genera un amplio conjunto de soluciones diferentes para el viento de una estrella supergigante $\mathrm{B}$, tal como se presenta en la sección 4.3.1. Por medio de las soluciones numéricas para $\rho(r)$ y $v(r)$, y a partir de los valores de $\dot{M}$ y de $\mathrm{v}_{\infty}$, se obtienen los perfiles de la línea $\mathrm{H} \alpha$. La grilla de modelos calculada es similar a la presentada en la tabla 4.4 excepto que, en este caso, se ha usado el parámetro $k$ $=0.20$ en lugar de 0.32. Para el parámetro $\alpha$ se ha considerado el valor fijo de 0.5. La figura 6.1 reúne una muestra de perfiles de $\mathrm{H} \alpha$ calculados para esos modelos, dispuestos de modo semejante a la tabla 4.4, con las filas alineadas según los valores de $\delta$, y las columnas asignadas a diferentes tasas rotacionales $\Omega$.

La línea roja da una referencia aproximada de la ubicación de la brecha que separa el régimen rápido (izquierda y arriba) del régimen lento (derecha y debajo). El casillero central de la muestra está vacío debido a que sus parámetros lo ubican justo en el interior de la brecha. Para las soluciones calculadas en el interior de la brecha, el código HYDWIND no alcanza la convergencia. A pesar de contar con soluciones del código ZEUS-3D para estos parámetros (sección 4.6.1), como se trata de soluciones discontinuas en el gradiente de velocidades (tipo quiebre o kink), el código FASTWIND tampoco logra la convergencia por lo cual no pueden derivarse los perfiles correspondientes.

Las diferencias de condiciones físicas entre ambos regímenes es reflejado claramente en el contraste entre los perfiles de línea generados a uno y otro lado de la brecha. Para la mayoría de los modelos en el régimen lento, los perfiles son de tipo P Cygni muy intensos, mientras que aquellos obtenidos para el régimen rápido son perfiles más pequeños con una componente en absorción predominante. Esto sucede, fundamentalmente, por los valores mayores de las tasas de pérdida de masa que prevalecen en los modelos del régimen lento, en comparación con el régimen rápido. De todos modos, también hay perfiles del régimen lento que están en absorción, especialmente aquellos que resultan de modelos con $\delta \sim 0.40$ y $\Omega \leq 0.4$.

Resulta directa la conexión de esta secuencia de perfiles con las relaciones entre $\dot{M}$ y $\delta$ del panel medio de la figura 4.10. La pendiente negativa en la relación entre $\dot{M}$ y $\delta$ causa una disminución en la componente en emisión del perfil P Cygni, que es originada por el decremento de la densidad, introducido colateralmente al aumentar el parámetro $\delta$. Esto puede observarse a lo largo de cada columna (para una velocidad de rotación fija). En el régimen rápido, de acuerdo a la figura 4.10, los valores sucesivos 
6.3. Análisis de los perfiles y los parámetros de la fuerza de radiación

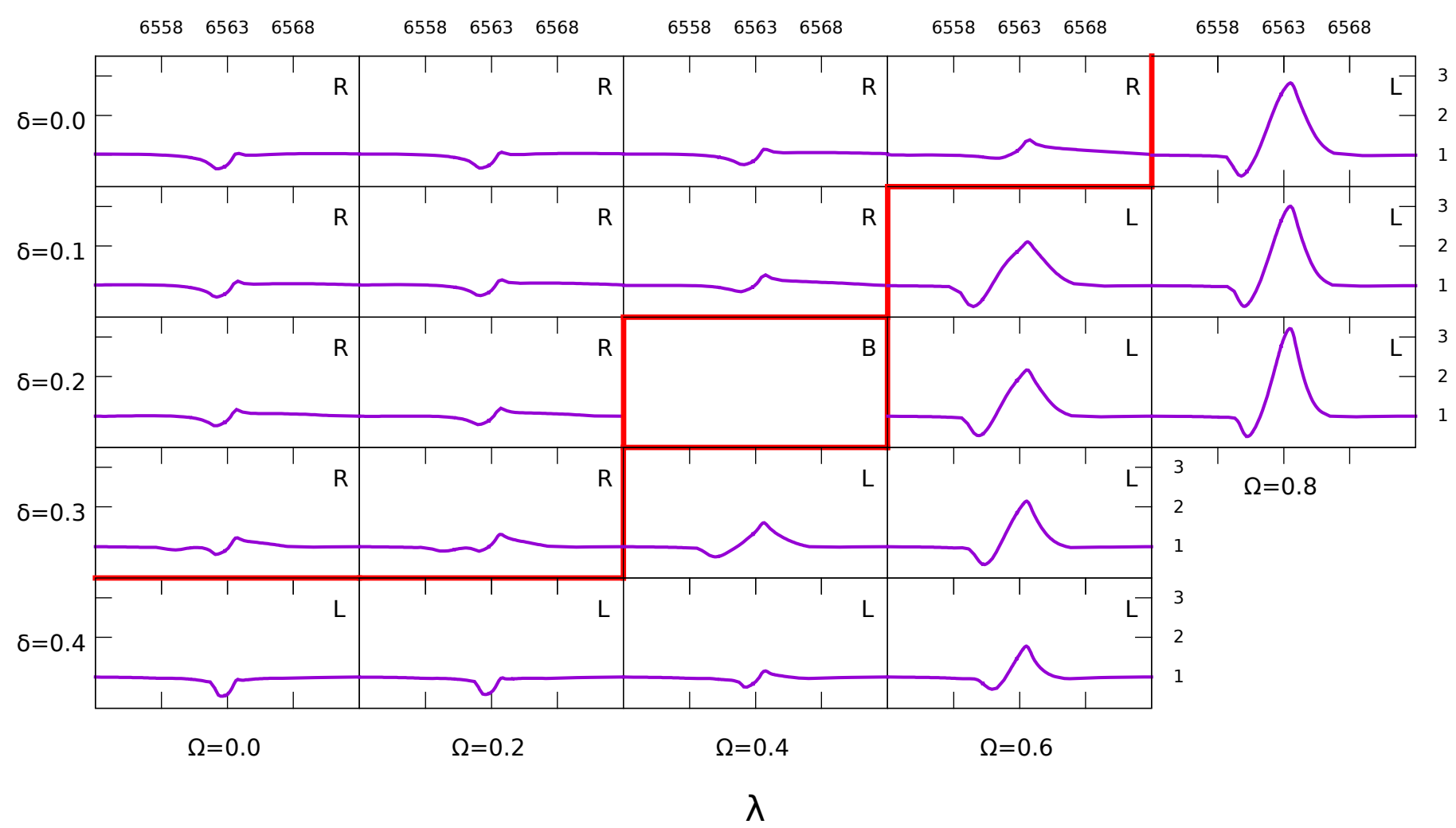

Figura 6.1. Muestra de perfiles de la línea $\mathrm{H} \alpha$ (flujo normalizado al continuo Flujo $/ \mathrm{F}_{\mathrm{c}}$ vs. longitud de onda $\lambda$ en $\AA$ ) para el modelo base T19, ordenados según $\delta$ (filas) y $\Omega$ (columnas). Los valores de $\alpha$ y $k$ son 0.5 y 0.2 , respectivamente. La línea roja indica la ubicación aproximada de la brecha. Los perfiles de línea ubicados por encima y a la izquierda de la brecha corresponden al régimen rápido (indicados con una letra "R"), mientras que el resto pertenece al régimen lento (letra "L"). El perfil correspondiente a $\delta=0.2$ y $\Omega=0.4$ correspondería exactamente al interior de la brecha (letra "B") y no se incluye (casillero vacío).

de $\delta$ para un valor pequeño de $\Omega$ generan tasas de pérdida de masa casi constantes. Por lo tanto, estos modelos pueden asociarse con las secuencias horizontales de perfiles que, prácticamente, son similares entre sí.

En el régimen lento, los valores altos en $\dot{M}$ producen una intensa emisión en los perfiles P Cygni pero, nuevamente, debido a la pendiente negativa de $\dot{M}$ para un dado valor de $\Omega$ (por ejemplo, $\Omega=0.6$ ), la componente en emisión se ve reducida a medida que $\delta$ crece.

Por lo tanto, para valores fijos de $k$ y $\alpha$ en el modelo T19, el régimen lento genera perfiles P Cygni muy intensos, a menos que la rotación sea suficientemente baja.

Finalmente, resulta interesante analizar los perfiles de $\mathrm{H} \alpha$ para modelos cuyos parámetros los ubiquen en proximidad de la brecha (borde extremo del dominio rápido en $\delta)$. En tales casos, las soluciones presentan un campo de velocidades que comienza a ser lentamente distorsionado en su curvatura (figura 4.4). Estos cambios en los gradientes 
de velocidades generan componentes en absorción dobles en los perfiles P Cygni. Un perfil con doble absorción como los mencionados, puede verse en la figura 6.1 para $\delta$ $=0.30$ y $\Omega$ entre 0 y 0.2 . La figura 6.2 superpone la fila de perfiles mencionados de la figura 6.1 en un único gráfico para facilitar la comparación. En este gráfico puede notarse la doble absorción (líneas rosa y azul) en los perfiles correspondientes a los valores de $\Omega$ de 0 y 0.2 .

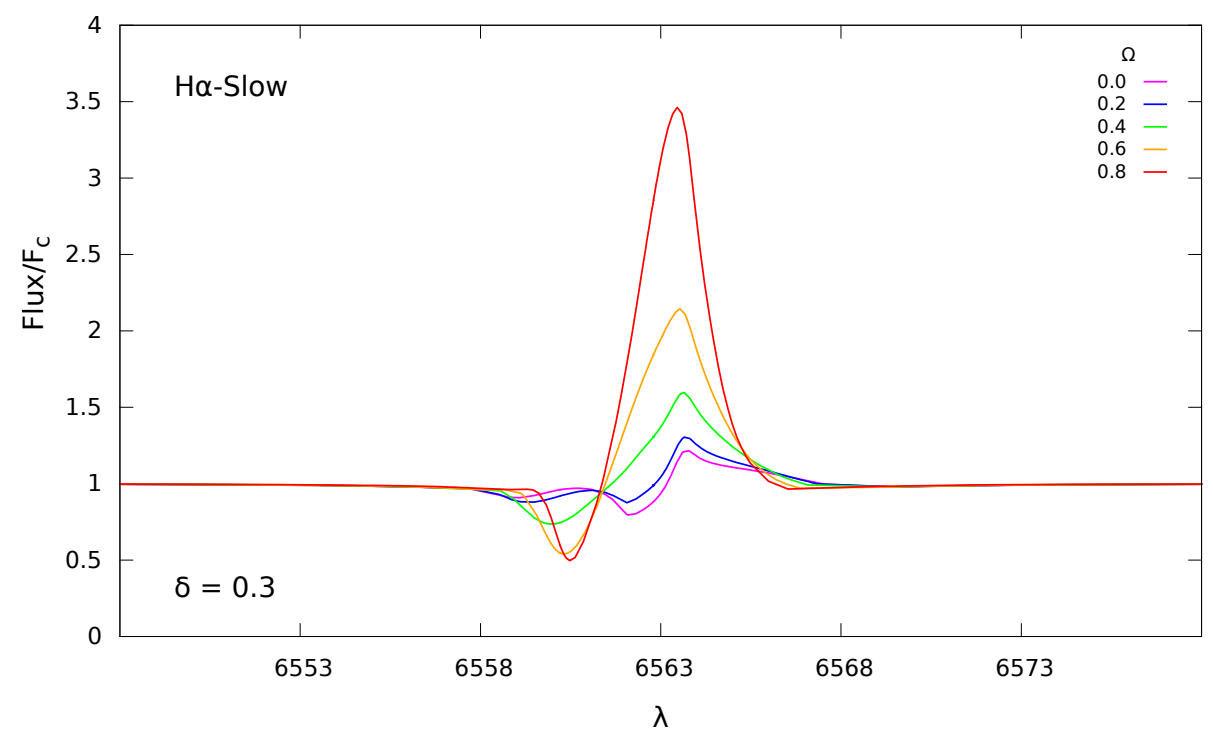

Figura 6.2. Perfiles de la línea $\mathrm{H} \alpha\left[\right.$ Flujo/F $\mathrm{F}_{\mathrm{c}}$ vs. $\left.\lambda\right]$ calculados para diferentes valores de $\Omega$ referenciados en el gráfico. Los perfiles de línea fueron obtenidos para el modelo base $\mathrm{T} 19$ con $k=0.20, \alpha=0.50$ y $\delta=0.3$ (es decir, para régimen lento). Nótese las componentes dobles en absorción para las bajas rotaciones (línea azul y magenta).

Cuando se analizaron las relaciones de escala para los vientos rotantes impulsados por radiación, en la sección 4.3.1, se notó que, a medida que se consideran valores mayores para la tasa rotacional $\Omega$, se alcanzan valores más grandes de $\dot{M}$. Esta dependencia también produce un aumento en las componentes en emisión de los perfiles. Las cantidades mayores de material expulsado en el viento aumentan la tasa de emisión en el ala roja del perfil P Cygni. Esta tendencia también se ilustra en la figura 6.2, que compara los perfiles de la línea $\mathrm{H} \alpha$ obtenidos para diferentes $\Omega$, ilustrando los efectos de esta variación sobre la componente en emisión.

Es importante notar que, en los perfiles de las figuras 6.1 y 6.2, sólo se ha tenido en cuenta el efecto que la rotación ejerce sobre la expansión del gas. En el cálculo de los perfiles no se ha incluido el efecto del ensanchamiento rotacional (los perfiles obtenidos con FASTWIND incluyen, por defecto, ensanchamiento Doppler y Stark). Los perfiles presentados en esta sección, no han sido convolucionados con un perfil rotacional, para evitar efectos que no fueren generados directamente por la fuerza de radiación en el viento. Tampoco se busca considerar la contribución de la rotación de la fotosfera sobre los perfiles sintéticos. 

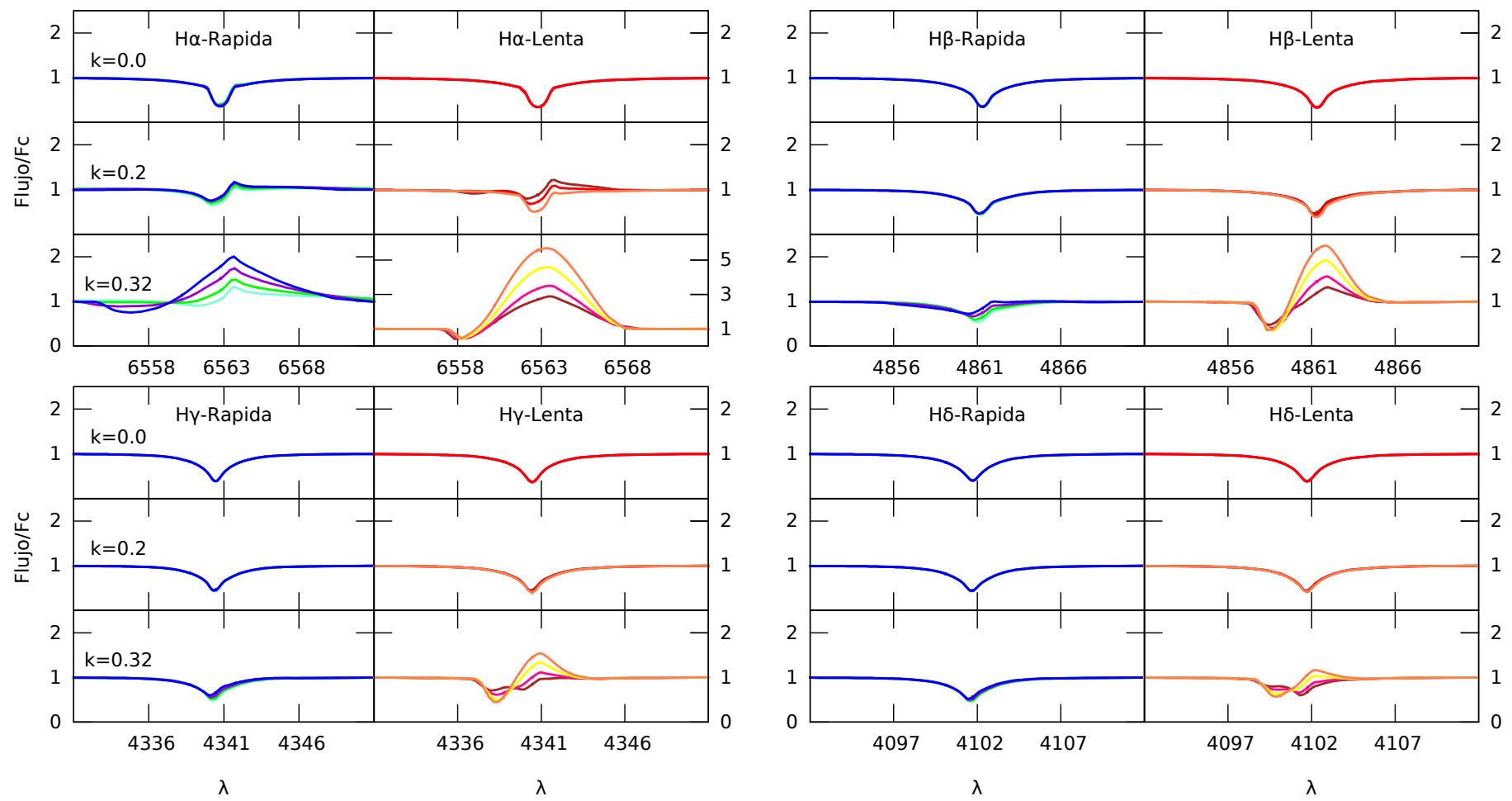

Figura 6.3. Perfiles de las líneas $\mathrm{H} \alpha, \mathrm{H} \beta, \mathrm{H} \gamma$ y $\mathrm{H} \delta$ [Flujo/F $\mathrm{F}_{\mathrm{c}}$ vs. $\lambda$ ] para el modelo base T19, comparando los resultados para los regímenes rápido y lento para $\Omega=0$. En cada fila se incluyen los perfiles calculados con los siguientes valores de $k: 0.10$, 0.20 y 0.32 . Para todos los modelos, $\alpha=0.5$. Cada valor de $\delta$ se identifica con un color de la línea. En el régimen rápido: 0 (celeste), 0.08 (verde), 0.16 (violeta) y 0.22 (azul). En el régimen lento: 0.30 (marrón), 0.34 (rosa), 0.36 (rojo), 0.38 (amarillo) y 0.40 (anaranjado).

\subsubsection{Dependencia de los perfiles de línea con $\delta$ y $k$}

La figura 6.3 permite comparar los perfiles sintéticos de las líneas $\mathrm{H} \alpha, \mathrm{H} \beta, \mathrm{H} \gamma$ y $\mathrm{H} \delta$ para el modelo base $\mathrm{T} 19$, obtenidos con los regímenes rápido (izquierda) y lento (derecha). Cada fila en los cuadros corresponde a un valor diferente de $k: k=0.10$ (fila de arriba), $k=0.20$ (fila intermedia) y $k=0.32$ (fila de abajo). Cada panel superpone los perfiles de línea calculados con diferentes valores de $\delta$, graficados en distintos colores (los valores de $\delta$ constan en el epígrafe de la figura).

Es evidente, a partir de la figura 6.3, que los valores bajos de $k$ sólo generan perfiles en absorción, sin presentar diferencias entre los originados por el régimen rápido o el régimen lento. Los perfiles tampoco presentan variaciones significativas ante cambios adicionales del parámetro $\delta$. La estimación de $\dot{M}$ a partir de perfiles de líneas obtenidos bajo estas condiciones, estaría afectada por grandes incertezas.

Por lo tanto, sería imposible discriminar, a partir de la examinación de perfiles en absorción, qué clase de régimen domina el viento en el caso en que el valor del parámetro $k$ fuese muy bajo. Esto sería característico de vientos con pocas líneas espectrales en capacidad de absorber momento del campo de radiación; es decir, vientos ópticamente delgados o en atmósferas pobres en metales. De esta manera, no resulta 
apropiado estudiar, con los medios con que se cuenta actualmente, entornos como los que caracterizan a los vientos débiles (sección 2.6.5).

La similitud en los perfiles en absorción implica, por lo tanto, que los vientos de estrellas supergigantes B más apropiados para ser ajustados con estos modelos de vientos rotantes impulsados por radiación, son los que presentan líneas en emisión en sus espectros. De otra manera, aunque pudieran ajustarse adecuadamente los perfiles de líneas en absorción, no sería posible extraer información sobre el estado físico del fluido, especialmente si se está tratando con un viento dominado por alguno de los regímenes de velocidades. Sumado a esto, en estas condiciones, no hay ninguna evidencia de cambios en la forma de los perfiles aún para los casos de cambios sustanciales en la estructura de la densidad (a través de $\delta$ ).

Estas afirmaciones son válidas, en principio, solamente en el rango óptico del espectro y con las líneas calculadas en el presente trabajo. Como lo demuestran Najarro et al. (2011), la utilización de líneas espectrales en la banda L (particularmente, la línea $\operatorname{Br} \alpha$ ) podría ser una herramienta eficaz de diagnóstico en estos casos (ver sección 1.1.1.

A diferencia del caso anterior, como puede verse en la figura 6.3, los valores altos de $k$ producen perfiles $\mathrm{P}$ Cygni intensos en la línea $\mathrm{H} \alpha$ para ambos regímenes, presentando en el régimen lento, las mayores emisiones. Para un valor fijo de $k$, la componente en emisión del perfil P Cygni crece a medida que aumenta $\delta$. Por lo tanto, en un régimen con valores altos de $k$ (por ejemplo, 0.30), la única manera de obtener componentes en emisión moderadas como las observadas en las supergigantes B, es que, simultáneamente, $\delta$ se mantenga con valores muy pequeños.

El parámetro $\delta$ tiene en cuenta la influencia de la ionización en la solución hidrodinámica del viento. Cuando esta solución hidrodinámica sirve como base para el cálculo de perfiles de línea, lo hace a través del campo de velocidades y de la distribución de densidad. El aumento en el parámetro $\delta$ implica la incorporación de una densidad adicional, a través del factor $\left(n_{e} / W\right)^{\delta}$ en la fuerza de radiación. Como la línea $\mathrm{H} \alpha$ es muy sensible a la densidad, dado que su opacidad depende cuadráticamente de $\rho$, el parámetro $\delta$ influye predominantemente en la intensidad de las componentes en emisión de los perfiles.

A continuación se examina la evolución del perfil de línea de $\mathrm{H} \alpha$ en el régimen rápido, al aumentar la ionización del medio, es decir, cuando $\delta$ crece (primer cuadro de la figura 6.3). Si el valor de $k$ no es demasiado pequeño (como en el caso citado en los párrafos anteriores), los perfiles $\mathrm{P}$ Cygni en medios con bajos niveles de ionización tienen componentes en absorción y emisión reducidas, muy próximas entre sí, dando un aspecto compacto en longitud de onda al perfil completo (línea celeste del panel izquierdo inferior de la figura 6.3 , con $k=0.32$ ). A medida que $\delta$ es incrementada (línea verde), la componente en absorción de ese perfil se rellena progresivamente con emisión proveniente del viento, debido a la densidad adicional. Cuando, progresivamente, los valores crecientes de $\delta$ van generando soluciones próximas a la brecha $(\delta \gtrsim 0.20$, línea violeta), surge una segunda componente en absorción localizada a una velocidad más alta, respecto a la componente previa de los perfiles. El perfil alcanza, entonces, su máxima extensión en longitud de onda, justo para modelos muy próximos a la brecha 
( $\delta=0.22$, línea azul). Este comportamiento se debe a las suaves distorsiones que aparecen en las leyes de velocidades de las soluciones cercanas a la brecha. Estos leves cambios pueden observarse en la figura 4.4 .
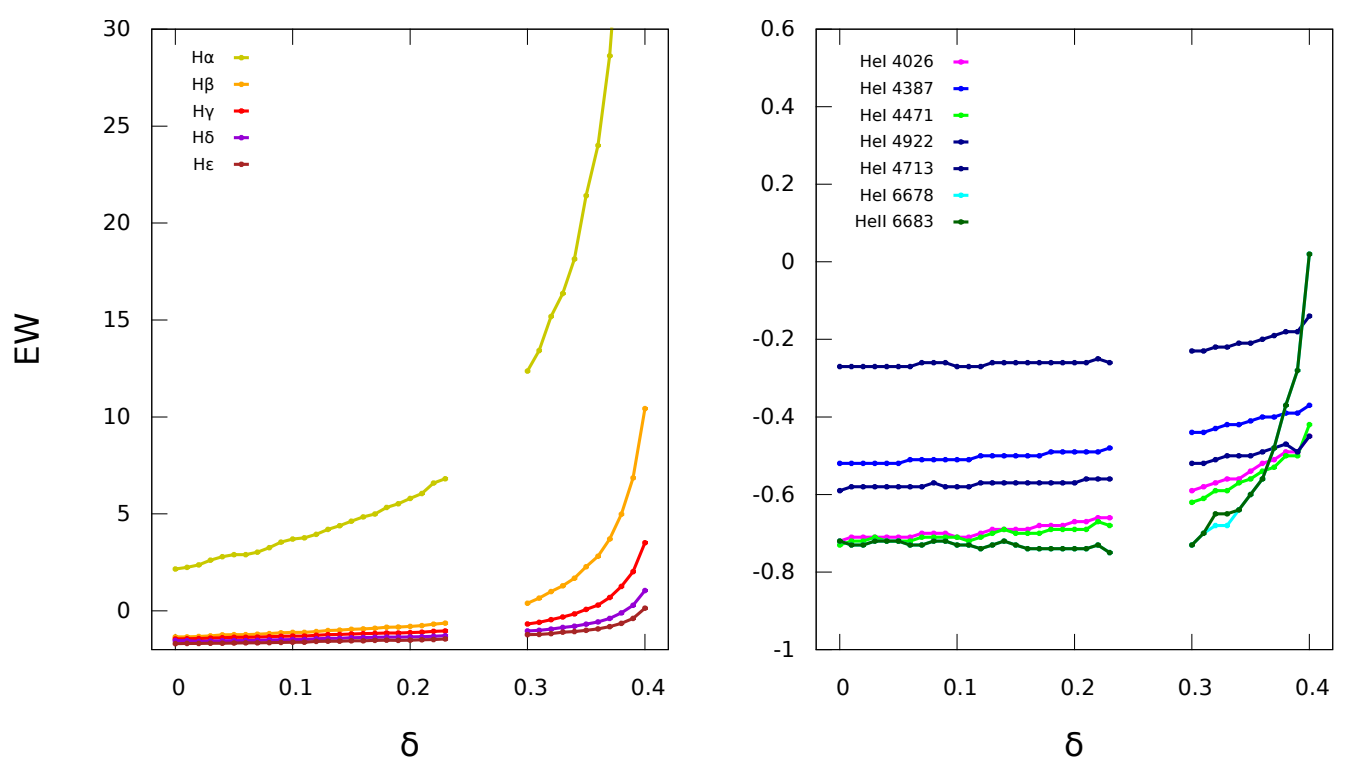

Figura 6.4. Izquierda: Anchos equivalentes (equivalent width, EW) en Å de las primeras cinco líneas de la serie de Balmer en función del parámetro $\delta$ (valores positivos indican mayor emisión). Nótese la extrema sensibilidad de la línea $\mathrm{H} \alpha$ en el régimen rápido. También, nótese que la curva podría continuarse, conectando los anchos equivalentes a ambos lados de la brecha, manteniendo el aspecto de la relación.

Derecha: Lo mismo para las líneas de He I y He II. Todos los valores fueron obtenidos con el modelo base T19, con $k=0.32$ y $\Omega=0$.

Cuando los valores de $\delta$ superan el límite superior de la brecha, $\delta_{\mathrm{b} 2}$, las soluciones ya se encuentran en el régimen lento. El perfil $\mathrm{P}$ Cygni resultante muestra mayor emisión que sigue creciendo conforme $\delta$ aumenta. Los perfiles son considerablemente más angostos en longitud de onda que en el caso del régimen rápido para $\delta$ alto.

Generalmente, las formas de los perfiles de línea que se originan en el régimen lento tienden a exhibir una mayor sensibilidad a cambios en la densidad, derivados de la ionización. En este régimen, los perfiles tienden a desarrollar, rápidamente, componentes en emisión más intensas que en el caso del régimen rápido, para cambios equivalentes en $\delta$.

Sorpresivamente, para $k=0.20$ (ver panel del medio de $\mathrm{H} \alpha$ en la figura 6.3), los perfiles de $\mathrm{H} \alpha$ generados en el régimen lento, se comportan de manera opuesta respecto al que se describe en los párrafos previos. Los perfiles comienzan como P Cygni para bajos valores de $\delta$ pero, a medida que $\delta$ crece, evolucionan perdiendo su componente en emisión y quedando sólo como un perfil en absorción. Sin embargo, esto es un resultado esperable ya que es consistente con el cambio de signo de la pendiente de $\dot{M}$ para $k=$ 0.20 , como se vio en la figura 4.10 . 

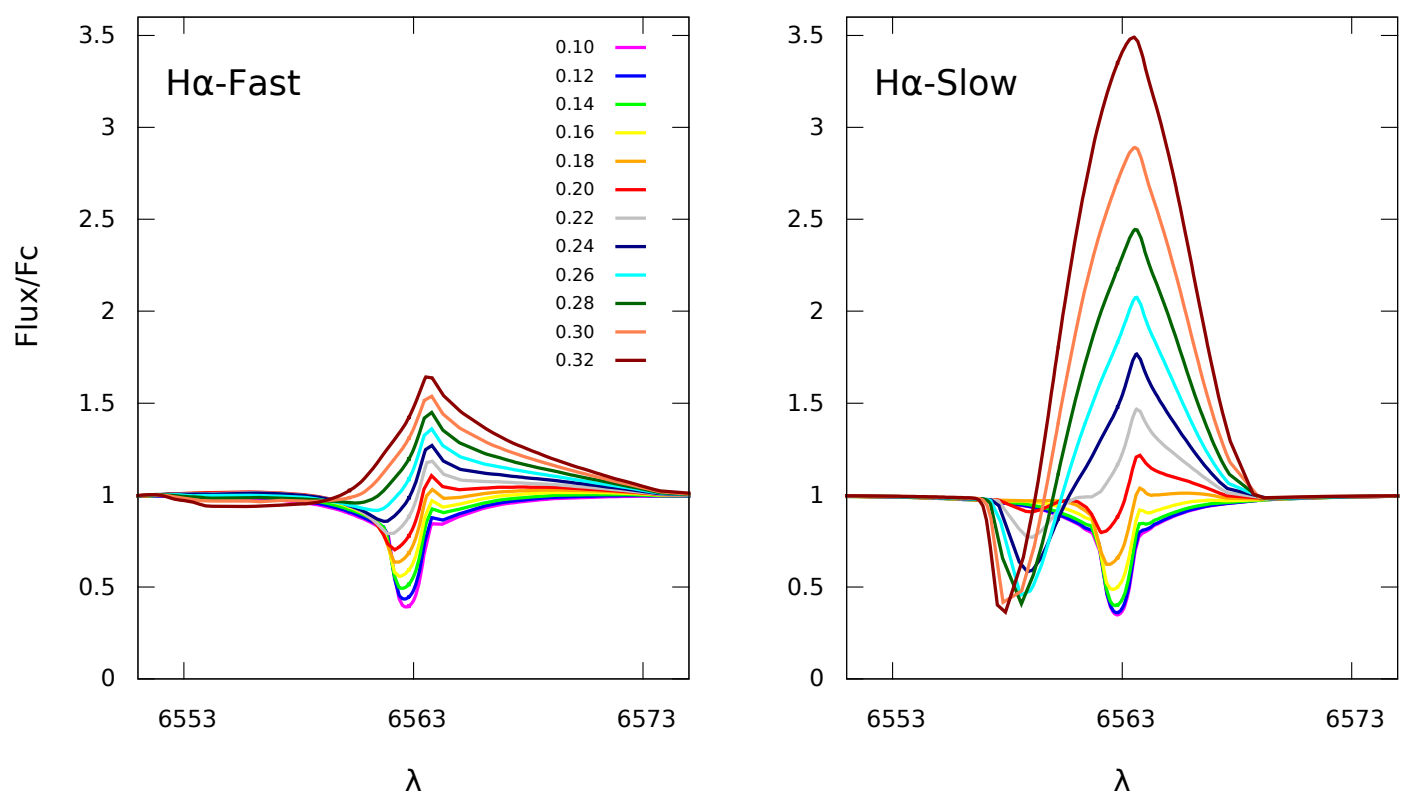

Figura 6.5. Izquierda: Perfiles de $\mathrm{H} \alpha$ [Flujo/F $\mathrm{F}_{\mathrm{c}}$ vs. $\left.\lambda\right]$ calculados para el modelo base T19, con diferentes valores de $k$, para un modelo no rotante. El resto de los parámetros se mantienen fijos a los valores $\alpha=0.5$ y $\delta=0.10$.

Derecha: Lo mismo que en el panel izquierdo, pero para $\delta=0.30$ (régimen lento).

En la figura 6.3, también puede verse que $\mathrm{H} \beta$ y $\mathrm{H} \gamma$ siguen un comportamiento semejante al de $\mathrm{H} \alpha$, conforme $\delta$ crece. Se puede notar que valores de $\delta \sim 0.4$ son excesivos, ya que generan demasiada emisión en $\mathrm{H} \gamma$ y $\mathrm{H} \delta$, la cual no es común en las supergigantes B.

Los anchos equivalentes de las líneas de baja excitación de la serie de Balmer muestran los mayores cambios ante variaciones del parámetro $\delta$, como puede verse en la figura 6.4. Este comportamiento es particularmente fuerte en el régimen lento. Por otro lado, los perfiles de las líneas de He I y He II (siendo las líneas del último ion muy débiles) reaccionan con cambios menores, como se muestra en el panel derecho de la figura 6.4. Esta figura también muestra una tendencia en los valores de los anchos equivalentes a ambos lados de la brecha, que parecerían continuar como si se tratara de una misma curva.

La figura 6.5 resume los resultados obtenidos para diferentes valores de $k$ en una secuencia detallada de perfiles de $\mathrm{H} \alpha$ calculada con un valor fijo de $\delta$ (en este caso, 0.10). Curiosamente, la sucesión de perfiles que resulta de la variación de $k$ resulta muy parecida a la generada en la variación de $\delta$ (figura 6.3) . La explicación a este parecido es que el crecimiento tanto de $k$ como de $\delta$ favorecen el aumento de la emisión en los perfiles. El parámetro $k$ está íntimamente ligado a la cantidad de líneas que impulsa al viento $y$, por ende, a la tasa de pérdida de masa. Un incremento en $k$ representa un aumento en la tasa de pérdida de masa, lo cual implica más material en condiciones de emitir fotones en $\mathrm{H} \alpha$. Por su parte, como ya se explicó, un aumento en la ionización 
aumenta la densidad del viento, lo que agrega emisión a $\mathrm{H} \alpha$.

\subsubsection{Dependencia de los perfiles de línea con $\alpha$}

La secuencia de paneles de la figura 6.6 muestra un conjunto de perfiles de $\mathrm{H} \alpha$ calculados para el modelo base T19, con valores fijos de $k=0.2$ y $\delta=0$, para diferentes valores de $\alpha$ y diferentes tasas de rotación. Los mismos están ordenados de modo similar a los de la figura 6.1 pero, en este caso, las filas corresponden a distintos valores del parámetro $\alpha$. Los perfiles de línea ubicados a la izquierda de la linea roja (ubicación aproximada de la brecha) corresponden al régimen rápido, mientras que los localizados en la última columna corresponden al régimen lento.

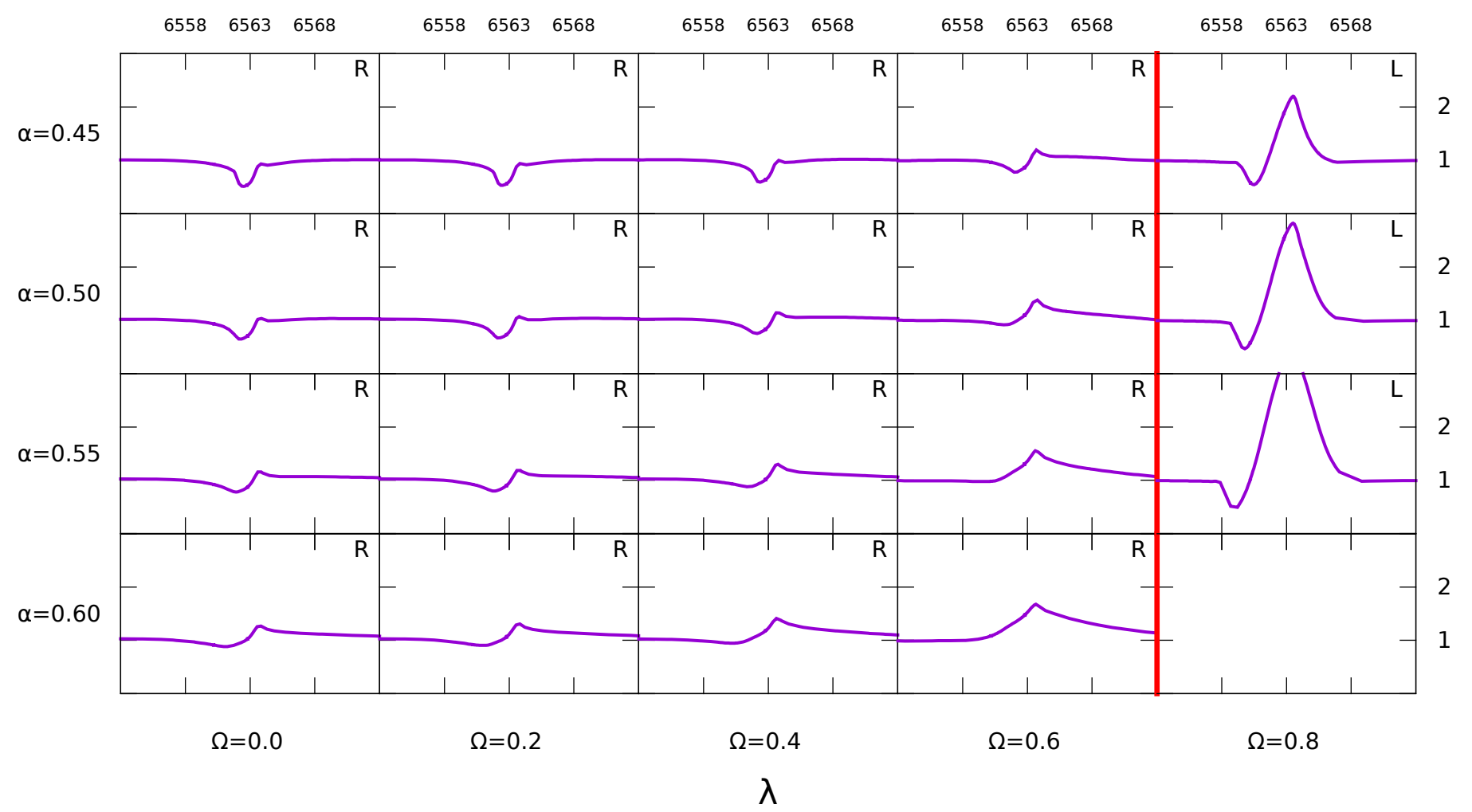

Figura 6.6. Muestra de perfiles de la línea $\mathrm{H} \alpha\left[\right.$ Flujo/ $\mathrm{F}_{\mathrm{c}}$ vs. $\left.\lambda\right]$ obtenidos para diferentes valores de $\alpha$ (filas) y tasas rotacionales (columnas). Los perfiles fueron obtenidos para el modelo base T19 con $k=0.20$ y $\delta=0$ (es decir, frozen in ionization). La línea roja indica la brecha, con el régimen rápido a la izquierda y el lento, a la derecha). El casillero para $\alpha=0.6$ y $\Omega=0.8$ se encuentra deliberadamente vacío, debido a la falta de convergencia en el modelo.

Los perfiles de línea desarrollan emisiones muy intensas conforme $\alpha$ se incrementa, y la cantidad de emisión también aumenta para altos valores de $\Omega$. Sin embargo, un comportamiento diferente respecto que se encontró para $k$, puede reconocerse en el gráfico. En este caso, a medida que crece $\alpha$, aumentan las componentes en emisión de los perfiles, pero simultáneamente se incrementa también la velocidad terminal. Este es un resultado esperado, que está de acuerdo a lo encontrado en la figura 4.12. En esta 
figura se muestra que $\mathrm{v}_{\infty}$ depende fuertemente del parámetro $\alpha$. Esto se hace evidente en el ancho, en longitud de onda, de cada perfil.

\subsection{Ajuste de los perfiles sintéticos a observaciones}

En esta sección se presenta la búsqueda, entre los régimenes de viento presentados, de modelos adecuados para representar las características observadas en los espectros de las estrellas supergigantes B, especialmente las líneas espectrales que pueden ser atribuidas al viento o que reciben una contribución significativa del mismo.

El modelado de líneas espectrales que se presenta en este trabajo se basa, casi exclusivamente, en el ajuste de los parámetros de la fuerza de radiación para conseguir perfiles sintéticos que mimifiquen los perfiles observados usando, a modo de marco de referencia, valores próximos a los suministrados en la literatura por otros autores, para los parámetros fundamentales de las estrellas. Esta modalidad de trabajo es, en esencia, la del item 2) de las estrategias de análisis presentadas en la sección 2.2.9.

Las limitaciones del método de trabajo seguido se deben, precisamente, a la imposibilidad de modificar los campos de velocidades a priori. Esto se hace, comúnmente, en la mayoría de los trabajos publicados (por ejemplo, entre muchos otros, Kudritzki et al. 1987; Markova \& Puls 2008; Lefever et al. 2010), en los cuales la forma de la ley de velocidades es fija, es decir una ley $\beta$ (ecuación 2.2), y los parámetros del viento $\left(\mathrm{v}_{\infty}\right.$ y $\beta$, junto a $\left.\dot{M}\right)$ son las variables de ajuste.

Por esta razón, la estrategia adoptada en este trabajo es considerablemente más difícil, ya que no siempre es posible encontrar conjuntos convenientes de los parámetros $(k, \alpha$ y $\delta)$ tales que generen soluciones convergentes adecuadas. Además, a partir de estas soluciones hidrodinámicas, la resolución de la ecuación de transporte de radiación debe ser capaz de producir perfiles de línea comparables con los observados. Este es un proceso lento y delicado de prueba y error, que demanda mucho trabajo y numerosas ejecuciones de los códigos de cálculo.

Sin embargo, las soluciones obtenidas por este método tienen la ventaja de ser más autoconsistentes que las encontradas forzando los parámetros y la forma del campo de velocidades. Resulta claro que, para lograr la autoconsistencia completa, se deberían calcular en un proceso iterativo, los parámetros de la fuerza de radiación a partir de los números ocupacionales que se deriven de los equilibrios de ionización y excitación, calculados detalladamente a lo largo de todo el viento. Este es un proceso que se ha llevado a cabo parcialmente, dado que implica avanzar en desarrollos muy complejos. La intención en este trabajo es evaluar si las soluciones discutidas son capaces de introducir observables, en primera instancia, antes de afrontar un proyecto de mayor magnitud.

\subsubsection{Criterios para el ajuste de los perfiles}

Para la selección de los modelos usados para el ajuste de los perfiles de línea se han tenido en cuenta los siguientes conceptos:

(a) Parámetros fundamentales de las supergigantes B: Como se introdujo en la sección 1.2.3, los parámetros fundamentales de las supergigantes B son esti- 
mados, comúnmente, mediante el ajuste de perfiles sintéticos a las observaciones (por ejemplo, Trundle et al. 2004; Markova \& Puls 2008; Lefever et al. 2010), eligiendo aquellas transiciones e iones que son especialmente sensibles a las propiedades que se busca determinar. Para estimar las temperaturas efectivas de las B supergigantes, es usual ajustar perfiles sintéticos a los observados en dos etapas contiguas de ionización, con objeto de dar cuenta del equilibrio de ionización preponderante, a través de algunas especies iónicas que tengan transiciones en las regiones espectrales para las que se cuenta con observaciones disponibles $(\mathrm{Ku}-$ dritzki \& Puls 2000). Entre las líneas espectrales apropiadas para llevar a cabo este procedimiento en el rango de temperaturas de las $\mathrm{B}$, se usan transiciones del silicio, tomando pares en estados de ionización contigua, tales como Si II con Si III, o Si III con Si IV, dependiendo del subtipo espectral (McErlean et al. 1999. Markova \& Puls 2008).
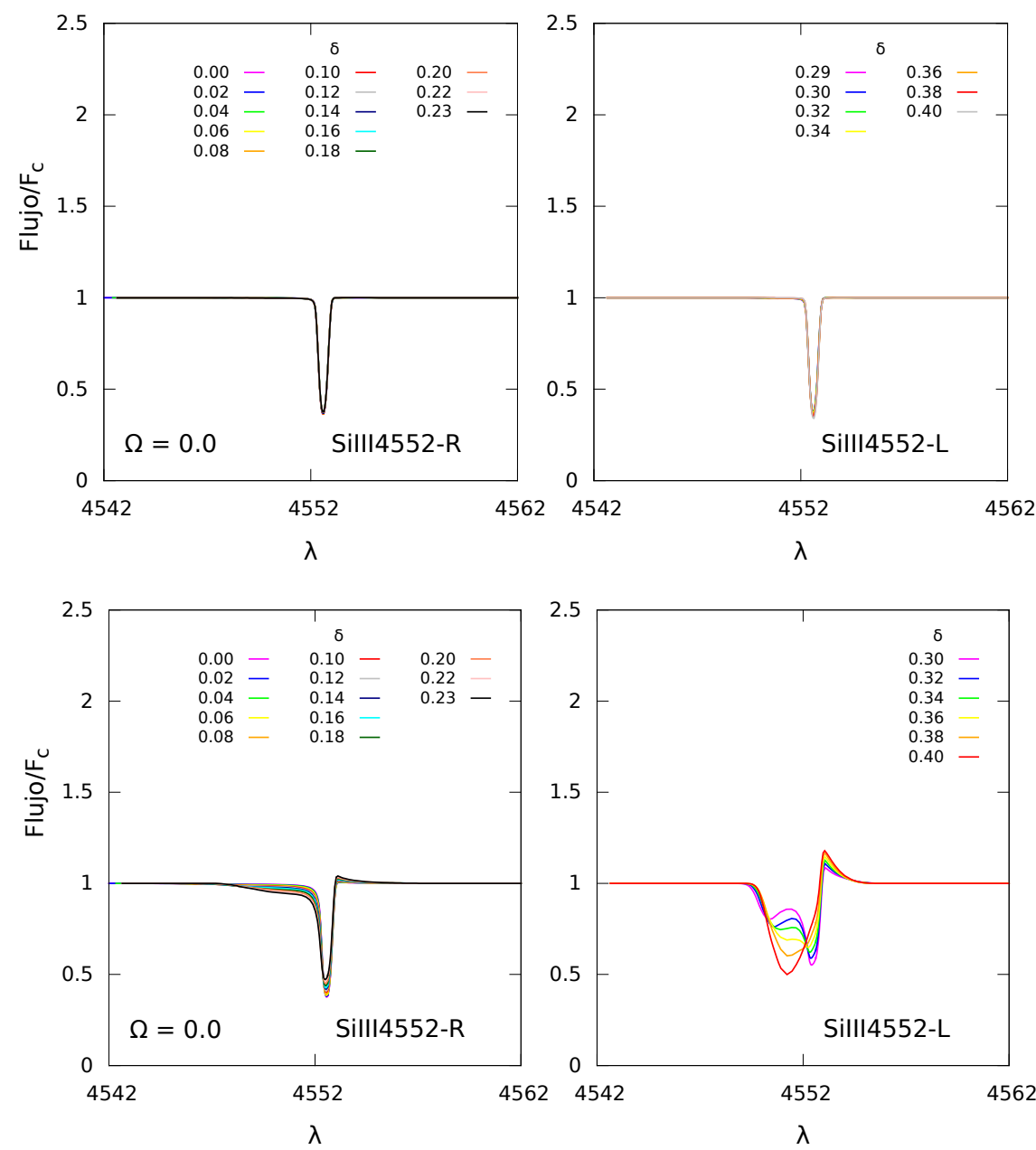

Figura 6.7. Arriba: Perfiles sintéticos de Si III $\lambda 4552 \AA\left[\right.$ Flujo/ $\mathrm{F}_{c}$ vs. $\left.\lambda\right]$ para el modelo base T19, calculado con los valores de $k=0.20$ y $\alpha=0.5$. A la izquierda se superponen los perfiles dados por la solución rápida mientras que, a la derecha, se encuentran los correspondientes a la solución lenta. Los valores de $\delta$ se muestran en la referencia. Abajo: Lo mismo, pero para $k=0.32$. 
Es importante remarcar que los perfiles sintéticos de las líneas de silicio obtenidos para la solución rápida y los obtenidos para la solución lenta, no presentan diferencias considerables, siempre que el parámetro $k$ no sea demasiado grande (por ejemplo, $k \sim 0.30$ ). El panel superior de la figura 6.7 muestra un ejemplo de este caso, con la coincidencia completa de los perfiles calculados para diferentes valores de $\delta$ para la línea Si III $\lambda 4552 \AA$, obtenidos con un parámetro $k=0.20$. En esta figura también puede verse que tampoco hay diferencias entre los perfiles generados en el régimen rápido (indicado con la letra "R") y los correspondientes al régimen lento (letra "L"). Esta secuencia de perfiles se corresponde con los presentados en la figura 6.3 para las líneas de Balmer (panel del medio para $k=$ 0.20). Si bien los perfiles de $\mathrm{H} \alpha$ parecen variar poco en esa figura, las intensidades alcanzadas en las emisiones son del orden de magnitud de las observadas en muchas supergigantes B. En este caso, estas pequeñas variaciones son realmente significativas para un ajuste de perfiles.
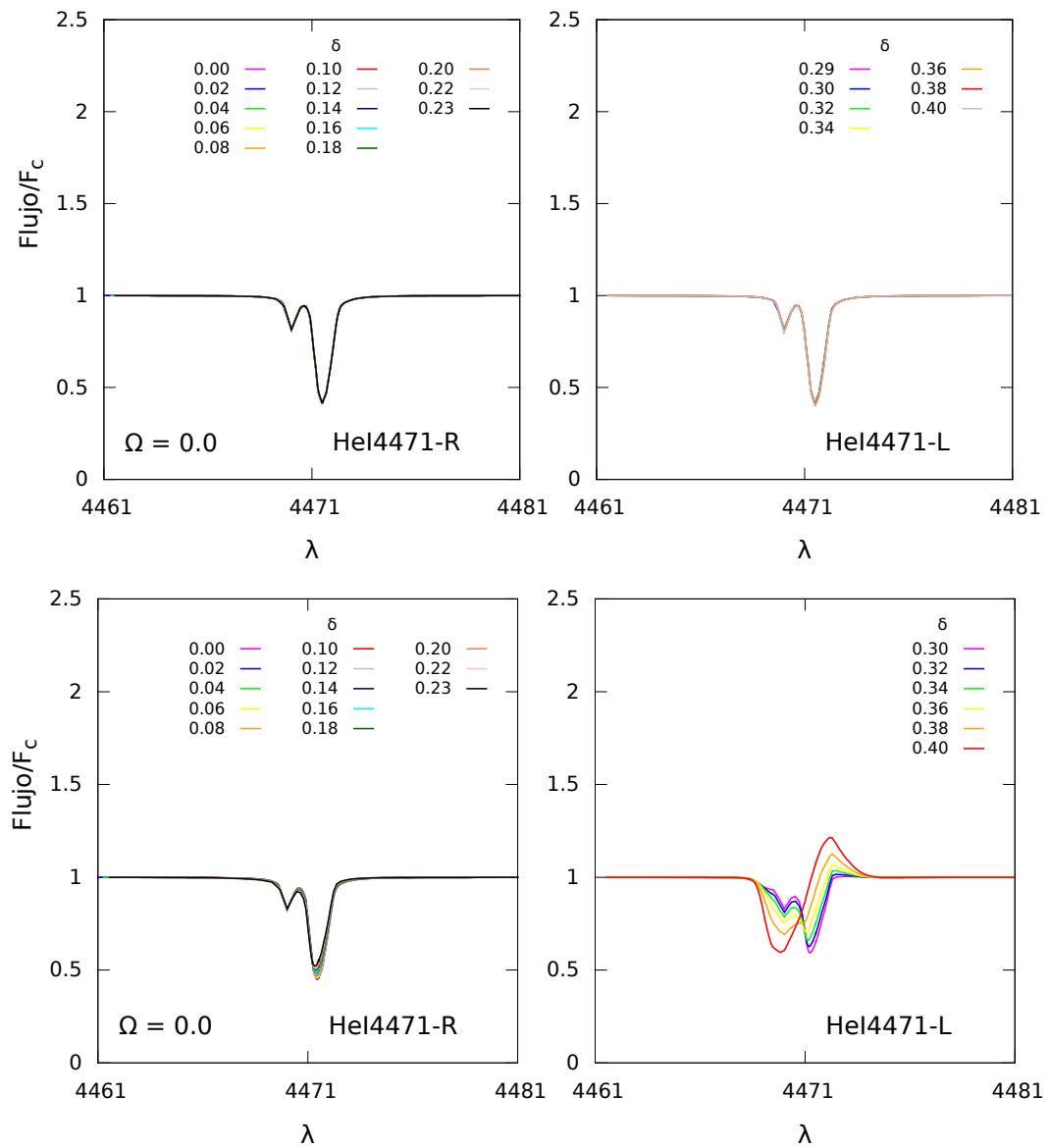

Figura 6.8. Similar a la figura 6.7 pero para He I $\lambda 4471 \AA$ A. Los paneles de arriba fueron calculados con valores $k=0.20$ y los de abajo, para $k=0.32$. La absorción situada a longitudes de onda más cortas, para cada perfil, es la componente prohibida que acompaña normalmente a esta línea en el rango espectral de la estrella.

La ausencia de variaciones significativas en los perfiles de las líneas de silicio es un resultado esperable, ya que la mayor parte de las líneas de ese elemento, en 
el visible, son formadas en la fotosfera estelar; razón por la cual son utilizadas para estimar las temperaturas efectivas. Estas líneas espectrales no reciben una contribución significativa del viento. Por contraste, las líneas UV del ion Si IV están completamente afectadas por el mismo.

Sin embargo, cuando el parámetro $k$ es suficientemente alto, estas líneas muestran diferencias considerables. En el panel inferior de la figura 6.7, puede verse una secuencia similar de perfiles de la línea de Si III $\lambda 4552 \AA$, pero calculada para $k=$ 0.32. En estos casos, las diferencias más notorias entre los perfiles calculados para diferentes valores de $\delta$, tienen lugar en el régimen lento (cuadro de la derecha). La sucesión de perfiles que surge de incrementar $\delta$ es muy semejante a la descripta para el caso de $\mathrm{H} \alpha$ (sección 6.3.2.

Las líneas de He I también se comportan de un modo semejante, como puede verse en la figura 6.8. En esta figura se muestra una superposición de perfiles organizados de forma similar a los presentados en la figura 6.7, pero en este caso, para los perfiles de la línea de He I $\lambda 4471 \AA$. Nuevamente, los paneles de abajo muestran que, para valores altos de $k$, en este caso 0.32 , los cambios en los perfiles son significativos. Esto indica que los efectos del viento, para esos casos, se observarían aún al nivel de la fotosfera.

Adicionalmente, vale la pena notar que la mayoría de las líneas del ion de He II son muy pequeñas o inexistentes, para el modelo base T19, razón por la cual no son consideradas en este trabajo.

Para la determinación de log g es usual ajustar líneas de las series de Balmer tales como $\mathrm{H} \gamma$ o $\mathrm{H} \delta$. Como se ve en la figura 6.3, estos perfiles son muy poco afectados por los parámetros $\delta$ y $k$, en tanto se mantengan valores moderados para estos parámetros, en especial $k$. Como en los casos anteriores, si $k$ es grande, las diferencias entre los perfiles calculados, tanto para el régimen rápido como para el lento, resultan ser significativas.

Entonces, para construir los modelos de ajuste a las observaciones en este trabajo, será preferible elegir parámetros de la fuerza de radiación con valores moderados, a fin de reproducir las observaciones. De esta manera, el viento sólo afectará a líneas tales como $\mathrm{H} \alpha$ o $\mathrm{H} \beta$, evitando que los perfiles de las líneas del Si o del He, que se usan de diagnóstico para determinar los parámetros fundamentales de la estrella, resulten contaminados por la emisión de radiación a lo largo del viento.

Como, en este trabajo, no se buscan determinar los parámetros fundamentales de las estrellas, sino estimar los parámetros propios del viento, el procedimiento consiste en adoptar los parámetros fundamentales publicados por otros autores, que son representativos de la estrella en estudio. Luego se procede a elegir, dentro del rango disponible de valores, los parámetros de la fuerza de radiación que generen aportes significativos para dar cuenta de los efectos del viento, pero que sean suficientemente leves como para no producir alteraciones importantes en las herramientas de diagnóstico de la temperatura efectiva, la gravedad superficial o el radio estelar.

Para los modelos presentados, esto significa evitar modelos con parámetros relativamente altos, especialmente $k$ (por ejemplo, $k=0.3$ ). Con esta condición, 
se aceptarán las estimaciones previas a los parámetros fundamentales. Como la intención es diagnosticar los parámetros del viento, se puede usar la línea $\mathrm{H} \alpha$ o $\mathrm{H} \beta$, las cuales podrán dar una información más adecuada sobre cuál es el régimen del viento en la estrella en consideración.

(b) Parámetros globales del viento: El régimen rápido se asemeja, en gran parte del viento, a una ley $\beta$ de velocidades (Araya et al. 2014). Por lo tanto, la expectativa es que los perfiles de línea derivados de la solución rápida sean consistentes con los análisis previos basados en la ley $\beta$ para el ajuste de perfiles observados. Sin embargo, el régimen lento aún no ha sido evaluado en profundidad, aunque se ha mostrado, preliminarmente, su capacidad de ajuste de sus perfiles de línea a espectros observados en el trabajo de Haucke et al. (2015). Sin embargo, con el fin de realizar una comparación de los perfiles obtenidos con uno u otro régimen, en condiciones semejantes, siempre que sea posible, se realizará un ajuste simultáneo utilizando ambas clases de soluciones.

En resumen, siguiendo estas consideraciones, es importante separar completamente las estrategias para diagnosticar los parámetros fundamentales de la estrella, de aquellas que dan información sobre las condiciones del viento.

De acuerdo a esto, en este trabajo, los ajustes se realizarán preferencialmente a la línea $\mathrm{H} \alpha$, intentando reproducir con el mayor detalle posible, las componentes en emisión de dichos perfiles, ya que allí se encuentra la contribución principal del viento (sección 1.1.1). Por otro lado, el análisis a desarrollar se abocará en la capacidad de los perfiles obtenidos, preferentemente, a partir del régimen lento, para dar cuenta de las características observadas en los espectros de algunas supergigantes B.

El código FASTWIND no incorpora el ensanchamiento rotacional al cálculo final de los perfiles (el cual es de gran importancia para la solución $\Omega_{\text {lenta, }}$ por ejemplo). Para realizar la comparación de los perfiles de línea sintéticos con los observados es necesario hacer la convolución del perfil resultante con un perfil rotacional adecuado, de acuerdo a la rotación estelar (considerando el valor de $v \sin i$ ). Esta convolución se realiza con rutinas externas a FASTWIND, las cuales están programadas en IDL (Interactive Data Language). De la misma manera se realiza la convolución con perfiles generados por macroturbulencia, en los casos en que sea necesario.

Es importante notar que, para que los perfiles incorporen los efectos de la rotación y la macroturbulencia, se han usado funciones de convolución que representan globalmente a estos fenómenos. Para realizar la convolución, se siguen los lineamientos presentados en los capítulos 17 y 18 de Gray (2005). De este modo, las convoluciones se realizan sobre el perfil ya formado, calculado por FASTWIND. Por lo tanto, en este proceso no se tiene en cuenta la influencia que cada región de formación del viento, con su rotación o macroturbulencia, podría tener sobre el perfil combinado final de la línea. También se debe considerar que no se tiene en cuenta el oscurecimiento al borde sobre el disco estelar, sino que se lo supone como uniformemente iluminado. 


\subsubsection{Selección de las observaciones}

No es el propósito de este trabajo realizar un análisis particular de estrellas individuales, ni indagar en los parámetros fundamentales de las mismas; sino evaluar la capacidad de las soluciones lentas para representar el viento de estrellas supergigantes B. La capacidad de estas soluciones teóricas para modelar el viento de algunas estrellas de esta clase, posibilitará la exploración del campo de aplicación de estos modelos. Para esto se han seleccionado 10 supergigantes B que han sido observadas oportunamente por el Lic. M. Haucke (FCAG - UNLP) y cedidas gentilmente para este trabajo. Las referencias básicas de las observaciones usadas de cada uno de estos objetos se encuentran en la tabla 6.2.

Las observaciones fueron obtenidas con el espectrógrafo REOSC en modo de dispersión cruzada, con el telescopio Jorge Sahade de 2.15 m. en el Complejo Astronómico El Leoncito (CASLEO), San Juan, Argentina. La resolución espectral R fue de alrededor de 12600 en la región de la línea $\mathrm{H} \alpha$, obtenido para redes de 400 l/mm (\# 580) y un detector CCD TEK 1024×1024 con una ganancia de $1.98 \mathrm{e}^{-} / \mathrm{adu}$. Las reducciones de los espectros fueron realizadas por el Lic. M. Haucke siguiendo los procedimientos estándar mediante rutinas IRAF1.

Se adiciona, además, un espectro de la estrella 55 Cyg (HD 198478), obtenido con el espectrógrafo Coudé en el telescopio de $2 \mathrm{~m}$ del Observatorio Perek de Ondřejov, República Checa, cedido por gentileza de la Dra. M. Kraus. Esta observación fue tomada con una red de $830.77 \mathrm{l} / \mathrm{mm}$ y con un detector CCD SITe de $2030 \times 800$. Con este detector se alcanzó una resolución espectral de $\mathrm{R}=13000$ en la región de $\mathrm{H} \alpha$, y un rango de longitudes de onda que va desde $6253 \AA$ hasta $6764 \AA$. La reducción de esta observación fue llevada a cabo por la Dra. M. Kraus, también mediante rutinas IRAF.

\begin{tabular}{llll}
\hline \hline $\begin{array}{l}\text { Nombre } \\
\text { de la estrella }\end{array}$ & HD & Tipo Espectral & $\begin{array}{l}\text { Fecha de observación } \\
\text { (año/mes/día) }\end{array}$ \\
\hline$\xi^{2}$ Ori & 41117 & B2 Ia & $2006 / 01 / 15$ \\
PU Gem & 42087 & B3 Ia & $2006 / 01 / 15$ \\
V731 Mon & 47240 & B1 Ib & $2006 / 01 / 15$ \\
V820 Cas & 52382 & B0.5 Ia & $2006 / 01 / 15$ \\
LN Vel & 74371 & B6 Iab/b & $2006 / 01 / 15$ \\
OP Vel & 75149 & B3 Ia & $2013 / 02 / 05$ \\
GX Vel & 79186 & B5 Ia & $2006 / 01 / 15$ \\
V519 Car & 92964 & B2.5 Ia & $2013 / 02 / 05$ \\
OP Vel & 99953 & B1/2 Iab/b & $2015 / 02 / 13$ \\
55 Cyg & 198478 & B3 Ia & $2010 / 07 / 16$ \\
\hline
\end{tabular}

Tabla 6.2. Estrellas observadas con sus tipos espectrales (tomados de la base de datos Simbad, Wenger et al. 2000) y su fecha de observación. En todos los casos se pudo realizar un ajuste de los perfiles de $\mathrm{H} \alpha$.

\footnotetext{
${ }^{1}$ IRAF es distribuido por el National Optical Astronomy Observatories, que es operado por la Association of Universities for Research in Astronomy, Inc., en acuerdo cooperativo con the National Science Foundation.
} 
La tabla 6.3 muestra los parámetros fundamentales extraídos de la literatura y empleados para modelar estas estrellas, junto a una comparación con los valores usados por otros autores. Es importante remarcar, nuevamente, que no se intentaron determinar los parámetros fundamentales (por ejemplo $\mathrm{T}_{\text {eff }}$, $\log \mathrm{g}$ o radio) a partir de los ajustes de perfiles. Por el contrario, con unas pocas excepciones, se adoptaron los valores próximos más convenientes para estas cantidades, en concordancia con los datos suministrados por los trabajos citados.

De esta manera, la propuesta consiste en obtener ajustes aceptables de los perfiles de la línea $\mathrm{H} \alpha$, cuya formación recibe importantes contribuciones de fotones a lo largo del viento, generados bajo las condiciones dadas a partir de los parámetros fundamentales ya determinados por otros autores para las estrellas en cuestión. Se busca caracterizar al viento a través de sus parámetros $\dot{M}$ y v $_{\infty}$, obtenidos a partir de los parámetros CAK de la fuerza de radiación, en especial, haciendo uso del régimen lento. 
Tabla 6.3. Parámetros fundamentales usados para modelar las estrellas supergiantes B y su comparación con valores de referencia publicados.

\begin{tabular}{|c|c|c|c|c|c|c|c|c|}
\hline estrella & $\begin{array}{l}\mathrm{T}_{\text {eff }} \\
{[\mathrm{kK}]}\end{array}$ & $\begin{array}{c}\log g \\
\operatorname{cgs}\end{array}$ & $\begin{array}{c}\mathrm{R} \\
{\left[\mathrm{R}_{\odot}\right]}\end{array}$ & $\begin{array}{c}\log \left(\mathrm{L} / \mathrm{L}_{\odot}\right) \\
{[\text { dex }]}\end{array}$ & $\mathrm{M} / \mathrm{M}_{\odot}$ & $\Omega$ & $\begin{array}{l}\mathrm{v} \sin \mathrm{i} \\
{\left[\mathrm{km} \mathrm{s}^{-1}\right]}\end{array}$ & Ref. \\
\hline \multirow[t]{5}{*}{ HD 41117} & 19.5 & 2.35 & 62.0 & 5.70 & 31.4 & 0.2 & 54 & Tesis \\
\hline & 19.5 & 2.25 & 61.7 & 5.70 & $\cdots$ & $\cdots$ & 40 & ku99 \\
\hline & 18.5 & $\ldots$ & 43.4 & $\ldots$ & 25.0 & $\ldots$ & $\ldots$ & kr01 \\
\hline & 19.0 & 2.35 & 61.9 & 5.65 & $\ldots$ & $\ldots$ & 72 & cr06 \\
\hline & 19.5 & 2.3 & 22.0 & $\ldots$ & $\ldots$ & $\cdots$ & $\cdots$ & ha17 \\
\hline \multirow[t]{4}{*}{ HD 42087} & 18.0 & 2.50 & 37.0 & 5.11 & 15.8 & 0.4 & 77 & Tesis \\
\hline & 20.5 & 2.50 & 35.2 & 5.30 & $\cdots$ & $\cdots$ & 60 & ku99 \\
\hline & 18.0 & 2.50 & 36.6 & 5.11 & $21^{*}$ & $\ldots$ & 71 & se08 \\
\hline & 19.0 & 2.45 & 63.0 & $\ldots$ & $\ldots$ & $\ldots$ & $\ldots$ & ha17 \\
\hline \multirow[t]{4}{*}{ HD 47240} & 19.5 & 2.40 & 40.0 & 5.32 & 14.7 & 0.2 & 143 & Tesis \\
\hline & 20.3 & $\cdots$ & 23.0 & 4.90 & $(21)$ & $\cdots$ & 103 & $\mathrm{mo04}$ \\
\hline & 19.0 & 2.40 & 27.0 & 4.93 & $\ldots$ & $\cdots$ & 94 & le07 \\
\hline & 19.0 & 2.40 & 36.0 & $\ldots$ & $\ldots$ & $\ldots$ & $\cdots$ & ha17 \\
\hline \multirow[t]{4}{*}{ HD 52382} & 20.8 & 2.50 & 20.0 & 4.83 & 46.2 & 0.2 & 56 & Tesis \\
\hline & 20.8 & $\cdots$ & 20.4 & $\cdots$ & 17.0 & $\cdots$ & $\cdots$ & kr01 \\
\hline & 23.0 & 2.71 & 20.0 & $\ldots$ & $\ldots$ & $\ldots$ & 56 & le10 \\
\hline & 23.5 & 2.50 & 20.0 & 5.05 & $\ldots$ & $\ldots$ & 50 & ha17 \\
\hline \multirow[t]{3}{*}{ HD 74371} & 13.5 & 1.90 & 70.0 & 5.17 & 14.2 & 0.2 & 38 & Tesis \\
\hline & 13.4 & 1.90 & $\cdots$ & $\cdots$ & 24.0 & $\cdots$ & 31 & fr10 \\
\hline & 13.7 & 1.80 & 75.0 & $\ldots$ & $\ldots$ & $\ldots$ & 30 & ha17 \\
\hline \multirow[t]{4}{*}{ HD 75149} & 16.0 & 2.05 & 40.0 & 4.97 & 6.55 & 0.2 & $\cdots$ & Tesis \\
\hline & 16.0 & 2.05 & 39.0 & 4.95 & $\cdots$ & $\cdots$ & 30 & le07 \\
\hline & 15.9 & 2.20 & $\ldots$ & $\ldots$ & 24 & $\cdots$ & 30 & fr10 \\
\hline & 16.0 & 2.10 & 70.0 & $\ldots$ & $\ldots$ & $\ldots$ & $\ldots$ & ha17 \\
\hline \multirow[t]{4}{*}{ HD 79186} & 15.5 & 2.00 & 62.0 & 5.30 & 14.0 & 0.2 & 38 & Tesis \\
\hline & 13.6 & $\cdots$ & 62.4 & $\cdots$ & 18.0 & $\cdots$ & $\cdots$ & la95 \\
\hline & 15.0 & $\ldots$ & $\ldots$ & $\cdots$ & $\ldots$ & $\cdots$ & $\cdots$ & pr10 \\
\hline & 15.8 & 2.00 & 69.0 & $\ldots$ & $\ldots$ & $\ldots$ & $\ldots$ & ha17 \\
\hline \multirow[t]{4}{*}{ HD 92964} & 18.0 & 2.10 & 48.0 & 5.34 & 10.6 & 0.2 & 60 & Tesis \\
\hline & 18.0 & 2.10 & 48.0 & $\cdots$ & $\cdots$ & $\cdots$ & 31 & le07 \\
\hline & 15.6 & 2.00 & $\ldots$ & $\ldots$ & 30.0 & $\cdots$ & 36 & fr10 \\
\hline & 18.0 & 2.20 & 73.0 & $\ldots$ & $\ldots$ & $\cdots$ & $\cdots$ & ha17 \\
\hline \multirow[t]{3}{*}{ HD 99953} & 19.0 & 2.50 & 40.0 & 5.27 & 18.5 & 0.1 & 74 & Tesis \\
\hline & 16.8 & 2.15 & 49.0 & $\cdots$ & 32.0 & $\cdots$ & & fr10 \\
\hline & 19.0 & 2.30 & 29.0 & $\ldots$ & $\ldots$ & $\ldots$ & $\ldots$ & ha17 \\
\hline \multirow[t]{2}{*}{ HD 198478} & 18.8 & 2.43 & 57.0 & 5.56 & 31.9 & 0.2 & 55 & Tesis \\
\hline & 17.5 & 2.10 & 32.0 & 5.31 & 11.0 & $\ldots$ & 39 & $\mathrm{ma08}$ \\
\hline
\end{tabular}


Table 6.3 (cont'd)

\begin{tabular}{|c|c|c|c|c|c|c|c|c|}
\hline estrella & $\begin{array}{l}\mathrm{T}_{\text {eff }} \\
{[\mathrm{kK}]}\end{array}$ & $\begin{array}{c}\log g \\
\operatorname{cgs}\end{array}$ & $\begin{array}{c}\mathrm{R} \\
{\left[\mathrm{R}_{\odot}\right]}\end{array}$ & $\begin{array}{c}\log \left(\mathrm{L} / \mathrm{L}_{\odot}\right) \\
{[\mathrm{dex}]}\end{array}$ & $\mathrm{M} / \mathrm{M}_{\odot}$ & $\Omega$ & $\begin{array}{l}\mathrm{v} \sin \mathrm{i} \\
{\left[\mathrm{km} \mathrm{s}^{-1}\right]}\end{array}$ & Ref. \\
\hline & 17.5 & 2.25 & 46.1 & 5.26 & $23.0^{*}$ & $\ldots$ & 61 & se08 \\
\hline & 18.8 & 2.47 & 57.0 & 5.57 & 30.0 & $\ldots$ & 55 & kr15 \\
\hline
\end{tabular}

Note. - Referencias: - na75: Nandy \& Schmidt (1975) - kr01: Krtička \& Kubát (2001) - mo04: Morel et al. (2004) - cr06: Crowther et al. (2006) - le07: Lefever et al. (2007) - ma08: Markova \& Puls (2008) - se08: Searle et al. (2008) - le10: Lefever et al. (2010) - fr10: Fraser et al. (2010) pr10: Prinja \& Massa (2010) - kr15: Kraus et al. (2015a) - ha17: Haucke, Cidale, Venero, Curé, \& Kraus (2017) - * indica masa evolutiva, () indica valor de referencia, calculado a partir de los datos publicados.

\subsubsection{Ajustes a las observaciones}

La tabla 6.4 muestra los valores de los parámetros de la fuerza de radiación usados para obtener los ajustes de la línea $\mathrm{H} \alpha$, junto con los parámetros globales del viento, en comparación con trabajos previos que usen la ley $\beta$ u otros cálculos hidrodinámicos.

La muestra está compuesta por estrellas que pueden agruparse en tempranas, intermedias y tardías. A continuación se revisan algunas características de las estrellas estudiadas, tomadas de la literatura, junto con comentarios sobre el ajuste logrado.

\section{Supergigantes B tempranas (B0 - B1)}

HD 52382: Esta estrella es miembro de la asociación Gem OB1, con un tipo espectral B1 Ib. Lamers et al. (1995) da un valor de la temperatura efectiva de 20800 $\mathrm{K}$, el cual es usado en este trabajo. Este valor es inferior al dado por Lefever et al. (2010), que lo ubica en $23000 \mathrm{~K}$. El valor de $\log$ g que se usa en este trabajo es un poco menor que el estimado por Lefever et al. (2010), lo cual favorece el ajuste para conseguir una mayor emisión en el perfil. Como en el trabajo de Lefever et al. (2010), el valor del radio adoptado es de $20 \mathrm{R}_{\odot}$, el que permite obtener un buen acuerdo entre el perfil de $\mathrm{H} \alpha$ observado y el calculado. La tasa rotacional $\Omega$ usada para esta estrella es de 0.2 .

El perfil observado de la línea $\mathrm{H} \alpha$ presenta sólo una componente de emisión pura. Los ajustes obtenidos en este trabajo, para los regímenes rápido y lento pueden verse en la figura 6.9. Básicamente se intenta reproducir la cantidad de emisión del perfil y, en lo posible, el ancho del mismo.

Tanto en el régimen rápido como en el lento, los parámetros del viento obtenidos en los ajustes son llamativamente bajos. La tabla 6.4 muestra que, en ambos regímenes, $\mathrm{v}_{\infty}$ es casi seis veces menor que el valor obtenido por Krtička \& Kubát (2001) y menos de la mitad del valor medido por Lamers et al. (1995) en las líneas del UV. Lo mismo 
Tabla 6.4. Parámetros del viento y de la fuerza de radiación para las supergiantes B modeladas y comparación con valores dados en la literatura. Los valores de $\mathrm{v}_{\infty}, \mathrm{v}_{\text {micro }}$

(velocidad de microturbulencia) y $\mathrm{v}_{\text {macro }}$ (velocidad de macroturbulencia) están expresadas en $\mathrm{km} \mathrm{s}^{-1} . \dot{M}$ está expresada en unidades de $10^{-6} \mathrm{M}_{\odot}$ año ${ }^{-1}$.

\begin{tabular}{|c|c|c|c|c|c|c|c|c|c|c|}
\hline Estrella & Sol. & $\mathrm{v}_{\infty}$ & $\dot{M}$ & $\beta$ & $k$ & $\alpha$ & $\delta$ & $\mathrm{V}_{\text {micro }}$ & $\mathrm{v}_{\text {macro }}$ & Ref. \\
\hline \multirow[t]{4}{*}{ HD 41117} & lenta & 216 & 0.42 & $\ldots$ & 0.15 & 0.49 & 0.38 & 10 & 11 & Tesis \\
\hline & ley $\beta$ & 500 & 0.85 & 1.0 & $\cdots$ & $\cdots$ & $\cdots$ & 20 & $\cdots$ & ku99 \\
\hline & ley $\beta$ & 510 & 0.90 & 2.0 & $\ldots$ & $\ldots$ & $\ldots$ & 10 & $\ldots$ & cr06 \\
\hline & ley $\beta$ & 510 & 0.19 & 2.0 & $\ldots$ & $\ldots$ & $\ldots$ & $\ldots$ & $\ldots$ & ha17 \\
\hline \multirow[t]{5}{*}{ HD 42087} & lenta & 222 & 0.14 & $\cdots$ & 0.23 & 0.50 & 0.33 & 10 & 0 & Tesis \\
\hline & rápida & 420 & 0.28 & $\cdots$ & 0.22 & 0.49 & 0.12 & 10 & 0 & Tesis \\
\hline & ley $\beta$ & 735 & 0.11 & 3.0 & $\cdots$ & $\cdots$ & $\cdots$ & 40 & $\cdots$ & ku99 \\
\hline & ley $\beta$ & 650 & 0.50 & 1.2 & $\ldots$ & $\ldots$ & $\ldots$ & 15 & $\ldots$ & se08 \\
\hline & ley $\beta$ & 260 & 0.40 & 2.0 & $\ldots$ & $\ldots$ & $\ldots$ & $\ldots$ & $\ldots$ & ha17 \\
\hline \multirow[t]{4}{*}{ HD 47240} & lenta & 216 & 0.13 & $\ldots$ & 0.12 & 0.50 & 0.30 & 25 & 2.2 & Tesis \\
\hline & rápida & 357 & 0.23 & $\ldots$ & 0.12 & 0.49 & 0.14 & 20 & 2.7 & Tesis \\
\hline & ley $\beta$ & 1000 & 0.17 & 1.5 & $\cdots$ & $\cdots$ & $\cdots$ & 15 & 55 & le07 \\
\hline & ley $\beta$ & 450 & 0.315 & 1.0 & $\ldots$ & $\cdots$ & $\cdots$ & $\ldots$ & $\cdots$ & ha17 \\
\hline \multirow[t]{5}{*}{ HD 52382} & lenta & $\overline{172}$ & 0.07 & $\overline{c \ldots}$ & 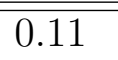 & 0.50 & 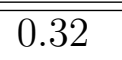 & 10 & 95 & $\overline{\text { Tesis }}$ \\
\hline & rápida & 208 & 0.09 & $\cdots$ & 0.12 & 0.485 & 0.21 & 10 & 95 & Tesis \\
\hline & medición & 550 & $\cdots$ & $\cdots$ & & $\cdots$ & $\cdots$ & $\cdots$ & $\cdots$ & la95 \\
\hline & sol. num. & 1030 & 0.49 & $\ldots$ & 0.451 & 0.514 & 0.091 & $\ldots$ & $\ldots$ & kr01 \\
\hline & ley $\beta$ & 1000 & 0.45 & 2.2 & $\ldots$ & $\ldots$ & $\ldots$ & $\cdots$ & $\cdots$ & ha17 \\
\hline \multirow[t]{2}{*}{ HD 74371} & lenta & 182 & 0.24 & 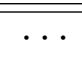 & 0.22 & 0.50 & 0.265 & 10 & 0 & Tesis \\
\hline & ley $\beta$ & 200 & 0.28 & 2.0 & $\cdots$ & $\cdots$ & $\ldots$ & $\cdots$ & $\cdots$ & ha17 \\
\hline \multirow[t]{4}{*}{ HD 75149} & lenta & 147 & 0.08 & $\cdots$ & 0.13 & 0.50 & 0.31 & 5 & 0 & Tesis \\
\hline & rápida & 175 & 0.10 & $\ldots$ & 0.13 & 0.48 & 0.20 & 10 & 0 & Tesis \\
\hline & ley $\beta$ & 500 & 0.10 & 2.5 & $\cdots$ & $\cdots$ & $\cdots$ & 15 & 60 & le07 \\
\hline & ley $\beta$ & 350 & 0.24 & 2.5 & $\ldots$ & $\ldots$ & $\ldots$ & $\ldots$ & $\ldots$ & ha17 \\
\hline \multirow[t]{2}{*}{ HD 79186} & rápida & 235 & 0.40 & $\cdots$ & 0.15 & 0.50 & 0.20 & 10 & 0 & Tesis \\
\hline & ley $\beta$ & 400 & 0.481 & 3.3 & $\cdots$ & $\cdots$ & $\cdots$ & $\cdots$ & $\cdots$ & ha17 \\
\hline \multirow[t]{4}{*}{ HD 92964} & lenta & 150 & 0.12 & $\cdots$ & 0.08 & 0.50 & 0.32 & 20 & 10 & Tesis \\
\hline & rápida & 173 & 0.16 & $\ldots$ & 0.08 & 0.48 & 0.21 & 10 & 18 & Tesis \\
\hline & ley $\beta$ & 520 & 0.28 & 3.0 & $\ldots$ & $\ldots$ & $\ldots$ & 15 & 50 & le07 \\
\hline & ley $\beta$ & 370 & 0.525 & 2.0 & $\ldots$ & $\ldots$ & $\ldots$ & $\ldots$ & $\ldots$ & ha17 \\
\hline \multirow[t]{3}{*}{ HD 99953} & lenta & 268 & 0.26 & $\cdots$ & 0.195 & 0.50 & 0.29 & 10 & 0 & Tesis \\
\hline & rápida & 406 & 0.50 & $\ldots$ & 0.22 & 0.50 & 0.20 & 10 & 0 & Tesis \\
\hline & ley $\beta$ & 250 & 0.107 & 2.0 & $\cdots$ & $\cdots$ & $\ldots$ & $\cdots$ & $\cdots$ & ha17 \\
\hline \multirow[t]{2}{*}{ HD 198478} & lenta & 284 & 0.49 & $\cdots$ & 0.18 & 0.50 & 0.277 & 20 & 0 & Tesis \\
\hline & ley $\beta$ & 250 & 0.24 & 2.0 & . & $\cdots$ & $\ldots$ & 50 & $\cdots$ & kr15 \\
\hline
\end{tabular}

Nota: sol. num.: solución numérica. Las ref. se corresponden con las citas de la tabla 6.3. 
sucede con $\dot{M}$, para la cual el valor calculado en este trabajo es también seis veces más bajo que el valor publicado (Krtička \& Kubát 2001).

El valor adoptado para $\alpha$ coincide con el usado por Krtička \& Kubát (2001), pero su valor de $\delta$ es bajo, en concordancia con lo estipulado por la teoría estándar, y claramente corresponde al régimen rápido. Además, el valor usado por estos autores para $k(0.451)$ produciría una emisión extremadamente intensa en los perfiles calculados a partir de las soluciones usadas en este trabajo.

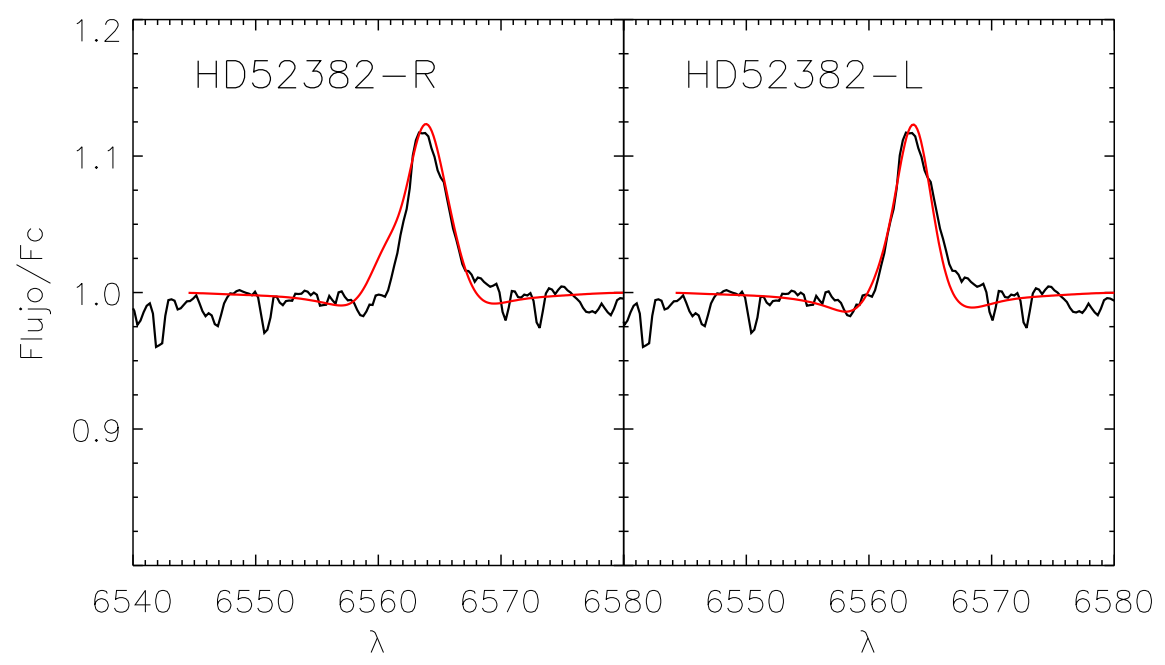

Figura 6.9. Ajustes del perfil de $\mathrm{H} \alpha$ calculados con soluciones rápida (izquierda) y lenta (derecha), para HD 52382.

Para poder ajustar el ancho del perfil en emisión, hubo que considerar un valor de $\mathrm{v}_{\text {macro }}=95 \mathrm{~km} \mathrm{~s}^{-1}$, el cual está de acuerdo con el valor obtenido por Haucke et al. (2017) usando la ley $\beta$. A pesar de que el ajuste obtenido en este trabajo (figura 6.9 podría considerarse como aceptable, los valores obtenidos para $\dot{M}$ y v v $_{\infty}$ resultan excesivamente bajos. Este valor alto de la velocidad de macroturbulencia es necesario para controlar la forma del perfil, manteniendo el valor de $v$ sin $i=56 \mathrm{~km} \mathrm{~s}^{-1}$ publicado por Lefever et al. (2007).

HD 47240: Se trata de una estrella de tipo espectral B1 Ib, la cual ha sido modelada por Lefever et al. (2007) con una temperatura efectiva de $19000 \mathrm{~K}$ y un valor de log g igual a 2.40. En este trabajo, se adopta una temperatura efectiva levemente superior, de $19500 \mathrm{~K}$ y el mismo valor para la gravedad superficial. Respecto al radio, Lefever et al. (2007) obtienen un valor de $27 \mathrm{R}_{\odot}$ pero, en este análisis, se encuentra que el mismo es demasiado bajo para reproducir algunas características del perfil observado. Para producir alas en emisión, como se observan en el perfil (figura 6.10), es necesario que el valor del radio sea mayor, en este caso de $40 R_{\odot}$. Respecto a la tasa rotacional $\Omega$, se adopta, como en la mayoría de los casos, un valor bajo de 0.2 .

El perfil observado muestra ambas alas en emisión y una absorción leve. La figura 6.10 muestra los ajustes de los regímenes rápido y lento para el mismo perfil. Si bien se puede obtener una forma semejante a la observada mediante los perfiles teóricos y se ajusta adecuadamente la absorción en ambos casos, las dos alas en emisión no se 


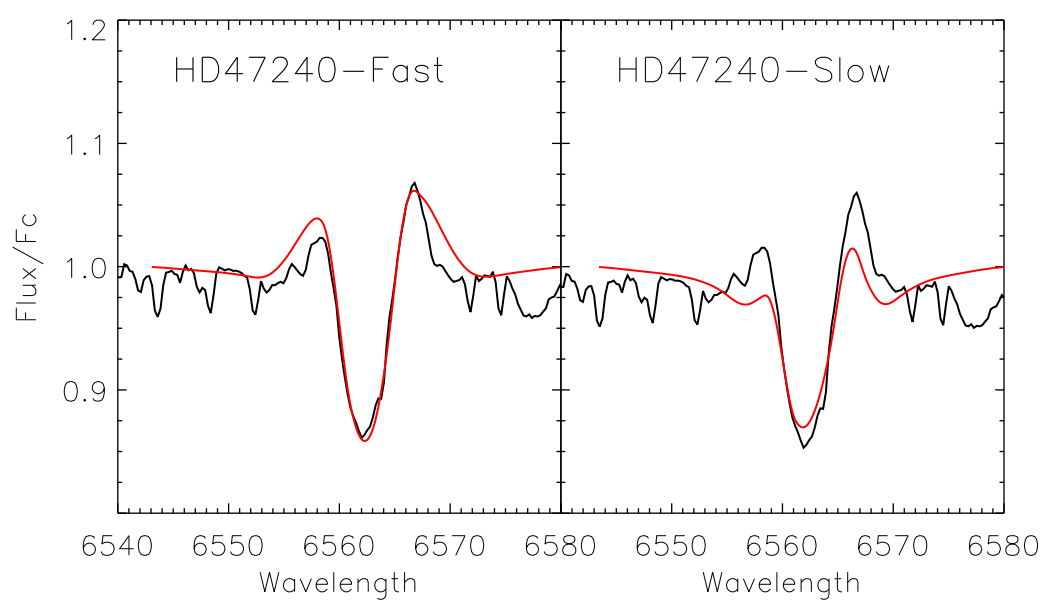

Figura 6.10. Ajustes del perfil de $\mathrm{H} \alpha$ para HD 47240.

pueden ajustar, simultáneamente, en su relación de intensidades.

Los valores derivados de $\mathrm{v}_{\infty}$ son inferiores a los obtenidos con ajustes de la ley $\beta$ de velocidades (Lefever et al. 2007), aún en el caso de la solución rápida. No obstante, hay un buen ajuste en el valor de $\dot{M}$. Para poder representar correctamente la forma de la absorción en el ajuste, se incrementó el ensanchamiento debido a la velocidad de microturbulencia a un valor de 20 y $25 \mathrm{~km} \mathrm{~s}^{-1}$ para el régimen rápido y para el lento, respectivamente. El valor de $v$ sin $i$ requerido para dar cuenta del ancho del perfil observado es superior al estimado por (Morel et al. 2004). Sin embargo, los datos de $v \sin i$ son inciertos en las supergigantes B y, comúnmente, sus valores son considerados del mismo orden que los correspondientes a la velocidad de macroturbulencia. Por este motivo, los valores de $v$ sin $i$ serán tomados de forma aproximada, de modo de mejorar el ajuste.

Los ajustes del perfil de $\mathrm{H} \alpha$ pueden considerarse como de regular calidad.

HD 99953: Es una estrella B1.5 Ia poco estudiada, sin estimaciones de las propiedades de sus vientos. Fraser et al. (2010) propusieron un valor de $\mathrm{T}_{\text {eff }}$ de $16800 \mathrm{~K}$. Sin embargo, ese valor no coincide con la calibración de los tipos espectrales, por lo que, en este trabajo, se usa $19000 \mathrm{~K}$. La gravedad superficial considerada es levemente mayor a la de Fraser et al. (2010), como puede verse en la tabla 6.3.

El perfil de $\mathrm{H} \alpha$ observado es de tipo $\mathrm{P}$ Cygni, con una posible componente en absorción en el ala azul. Tanto la solución rápida como la solución lenta son capaces de reproducir la forma general de la línea, como se observa en la figura 6.11. Sin embargo, la solución lenta también puede reproducir la posible componente en absorción en el ala azul. Eso se debe a que el modelo empleado para el régimen lento se encuentra próximo a la brecha y, en tales casos, los perfiles pueden mostrar componentes en absorción adicionales. Por otro lado, la solución lenta ajusta mejor el ancho de la componente en emisión del perfil P Cygni.

Prinja et al. (1990) asignaron a esta estrella un valor de $\mathrm{v}_{\infty}=510 \mathrm{~km} \mathrm{~s}^{-1}$, como una medición de tipo $\mathrm{v}_{\text {black }}$ (realizada en el borde azul de la componente en absorción 


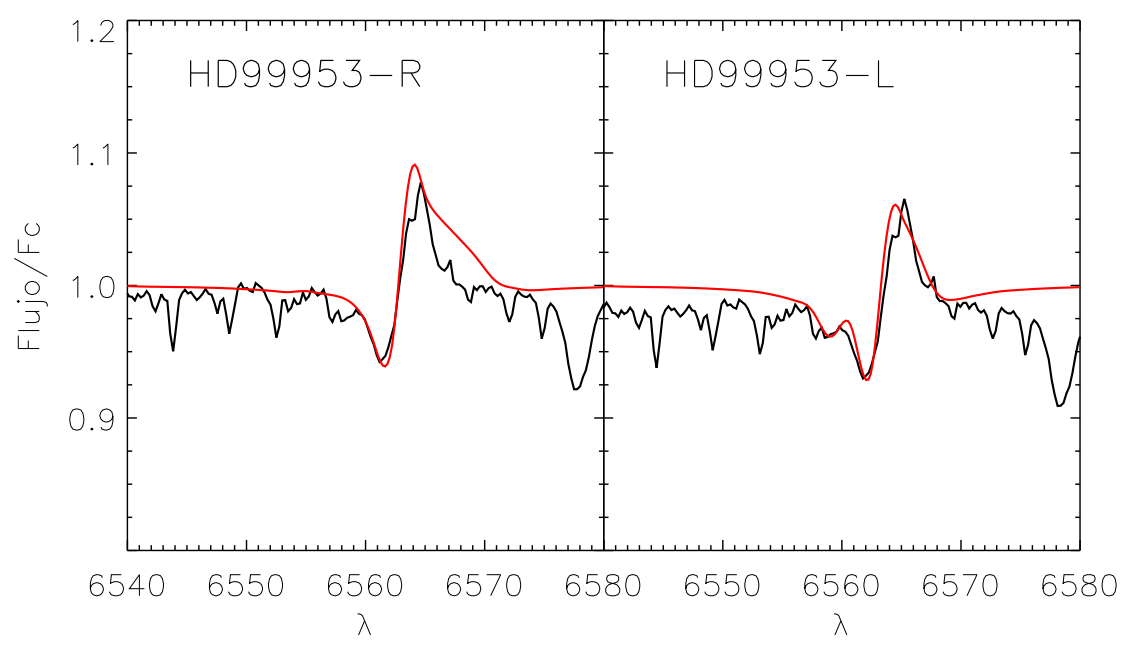

Figura 6.11. Ajustes del perfil de H $\alpha$ para HD 99953.

completamente saturada del perfil $\mathrm{P}$ Cygni). La solución rápida da un valor de $\mathrm{v}_{\infty}$ que dista en $100 \mathrm{~km} \mathrm{~s}^{-1}$ de esta medición. Para el régimen lento, la velocidad terminal encontrada es, aproximadamente, la mitad de la propuesta por Prinja et al. (1990). Hasta el momento, no hay mediciones de la tasa de pérdida de masa en la literatura. Los modelos en el régimen rápido, ajustados en este trabajo, dan un valor de 0.5 $\times 10^{-6} \mathrm{M}_{\odot}$ año ${ }^{-1}$. Para el régimen lento corresponden a la mitad de este valor.

Considerando que el perfil sintético reproduce en buen grado el perfil observado y que la solución lenta podría dar cuenta de la presencia de una segunda componente en absorción, se considera el ajuste como satisfactorio.

\section{Supergigantes B intermedias (B1.5 - B3)}

HD 41117: Es una estrella de tipo B2 Ia, miembro de la asociación Geminorum OB1, y ha sido intensamente estudiada. Las estimaciones de su $\mathrm{T}_{\text {eff }}$ la sitúan en un rango comprendido desde $18000 \mathrm{~K}$, obtenido por Barlow \& Cohen (1977) a partir de los excesos libre-libre en el IR intermedio, hasta $20640 \mathrm{~K}$, obtenido por medio del método BCD (Zorec et al. 2009). En este trabajo se usan valores próximos a los parámetros fundamentales estimados por Crowther et al. (2006) y Kudritzki et al. (1999), ya que estos trabajos realizan un ajuste a la línea $\mathrm{H} \alpha$.

Crowther et al. (2006) y Kudritzki et al. (1999) predicen para el viento, un valor de $\mathrm{v}_{\infty}$ unas 2.3 veces mayor que el valor encontrado para el régimen lento. Respecto a $\dot{M}$, sus valores duplican el valor encontrado en este trabajo.

Barlow \& Cohen (1977) estimaron un valor aún mayor para $\mathrm{v}_{\infty}$ acompañado también de un valor más elevado para $\dot{M}$. Sus resultados son semejantes a los encontrados por Morel et al. (2004) quienes determinaron, para $\mathrm{T}_{\text {eff }}=18000 \mathrm{~K}, \dot{M}=2.36$ $\times 10^{-6} \mathrm{M}_{\odot}$ año ${ }^{-1} \mathrm{y} \mathrm{v}_{\infty}=510 \mathrm{~km} \mathrm{~s}^{-1}$. A partir de la medición de excesos de emisión en radio, Benaglia et al. (2007) obtuvo un valor de $\dot{M}=2.1 \times 10^{-6} \mathrm{M}_{\odot}$ año ${ }^{-1}$.

Por su parte, Krtička \& Kubát (2001) obtuvieron la estructura del viento usando a los parámetros de la fuerza de radiación como punto de partida. Sus valores para $\mathrm{v}_{\infty}$ y $\dot{M}$ son mayores aún que los derivados del ajuste de $\mathrm{H} \alpha$ Kudritzki et al. 1999 . 


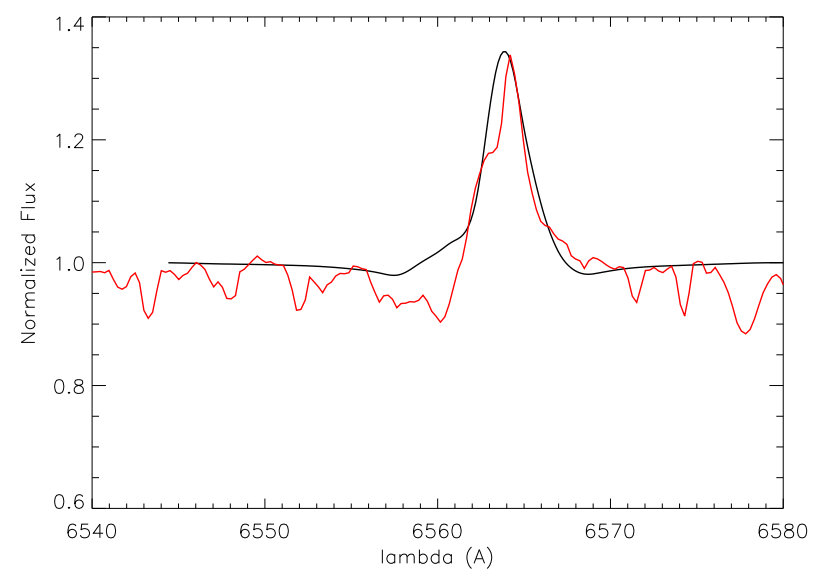

Figura 6.12. Ajuste de perfil de $\mathrm{H} \alpha$ para HD 41117 usando el régimen lento. Los parámetros correspondientes están en las tablas 6.2 and 6.3 .

Crowther et al.2006), y son tres y seis veces mayores que los resultados de este trabajo, respectivamente. Estos valores claramente muestran un escenario de viento en régimen rápido, en los que altos valores de $k$ producen intensas tasas de pérdida de masa e incrementan las componentes en emisión de los perfiles. Sin embargo, sus bajos valores de $\delta$ evitan el desarrollo de perfiles $\mathrm{P}$ Cygni muy intensos, como puede comprobarse en la figura 6.1 .

El ajuste del perfil $\mathrm{H} \alpha$ (figura 6.12) es aceptable ya que puede reproducir la componente en emisión aunque falla en la componente en absorción que es muy poco profunda. Las características del régimen lento pueden dar cuenta de la cantidad de radiación emitida en el viento. Para este ajuste, el valor de $\delta$ sitúa al modelo lejos de la brecha; es decir, se trata de un régimen lento bien establecido. No fue posible ajustar este perfil de línea con los modelos en el régimen rápido.

HD 42087: Es una estrella clasificada como B2.5 Ib con un valor de $\mathrm{T}_{\text {eff }}$ comprendido entre $18000 \mathrm{~K}$, obtenido por Searle et al. (2008) a partir del ajuste de perfiles de línea con el código CMFGEN (sección 5.2), y un $17440 \mathrm{~K}$ estimado mediante la calibración BCD por Zorec et al. (2009).

Benaglia et al. (2007) obtuvo un valor menor que $1.7 \times 10^{-6} \mathrm{M}_{\odot}$ año ${ }^{-1}$ para $\dot{M}$ mientras que Morel et al. (2004) y Searle et al. (2008) concordaron con un valor más bajo, de $0.2 \times 10^{-6} \mathrm{M}_{\odot}$ año ${ }^{-1}$. El valor de $M$ obtenido en este trabajo para la solución $\delta_{\text {lenta }}$ es $0.14 \times 10^{-6} \mathrm{M}_{\odot}$ año ${ }^{-1}$, unas 1.4 veces menor que el último valor citado. Respecto a $\mathrm{v}_{\infty}$, los valores obtenidos en este trabajo son tres veces más bajos que el menor valor dado por los trabajos citados.

A pesar de estas diferencias, puede verse en la figura 6.13, que el ajuste obtenido con la solución lenta es satisfactorio, representando simultáneamente la proporción de emisión y absorción del perfil P Cygni, y ajustando los anchos de ambas componentes. El ajuste conseguido con el régimen lento es más apropiado que el obtenido con el régimen rápido, cuyas alas están extendidas y no fue posible reducirlas.

HD 75149: La mayoría de los trabajos previos le asignan una temperatura efectiva cercana a $16000 \mathrm{~K}$ a esta, poco estudiada, estrella B3 Ia. En este trabajo se adoptan 


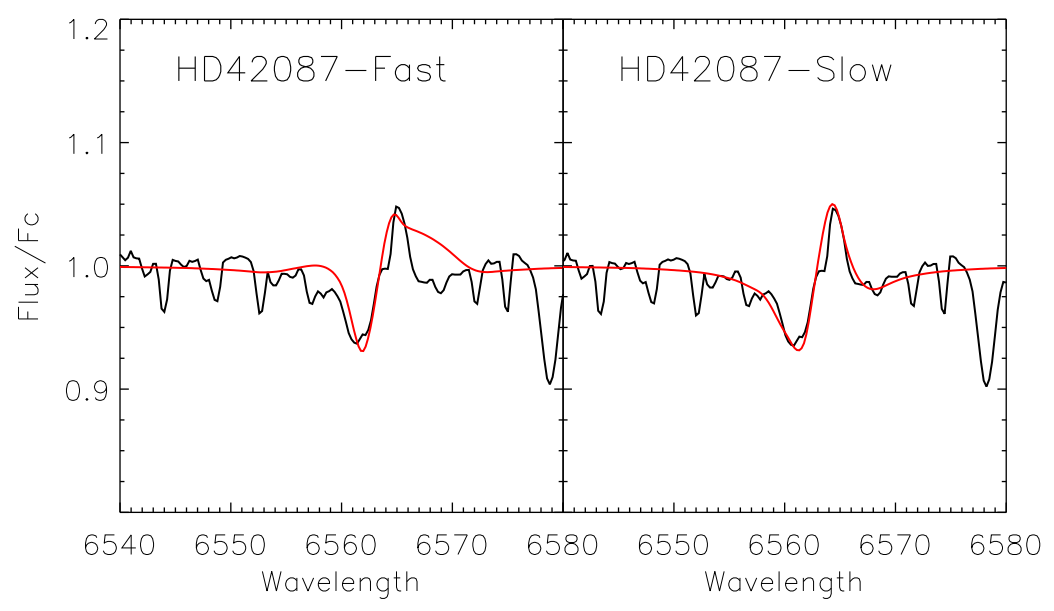

Figura 6.13. Ajustes de perfil de $\mathrm{H} \alpha$ calculados con soluciones rápida (fast, izquierda) y slow, lenta (derecha), para HD 42087.

como parámetros fundamentales los valores estimados por Lefever et al. (2007).

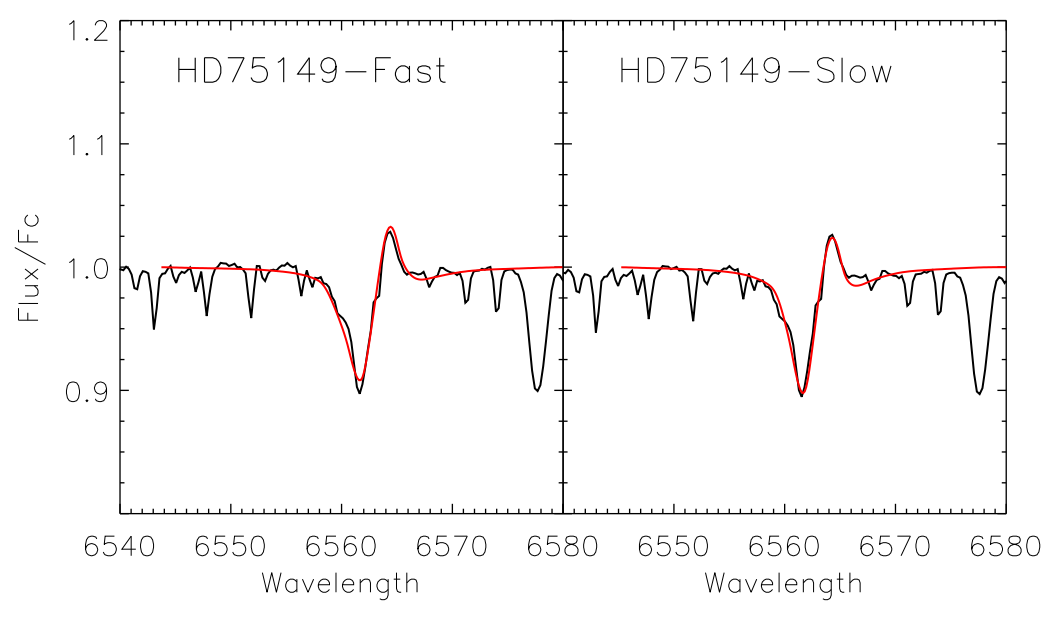

Figura 6.14. Ajustes del perfil de $\mathrm{H} \alpha$ para HD 75149.

Los ajustes logrados en este trabajo son excelentes, como puede verse en la figura 6.14. El perfil de $\mathrm{H} \alpha$ es reproducido con buena aproximación tanto con el régimen rápido como con el régimen lento.

Se obtiene un valor de $\dot{M}$ muy cercano al de Lefever et al. (2007). Sin embargo, el valor de $\mathrm{v}_{\infty}$ estimado aquí es más bajo en un factor tres o más. Howarth et al. (1997) encontraron un valor para $\mathrm{v}_{\infty}$ de $350 \mathrm{~km} \mathrm{~s}^{-1}$ derivado a partir de mediciones en los perfiles de línea del UV. Ese valor es considerablemente menor que el valor $\left(500 \mathrm{~km} \mathrm{~s}^{-1}\right)$ encontrado por Lefever et al. (2007). Es conveniente mencionar que, para 


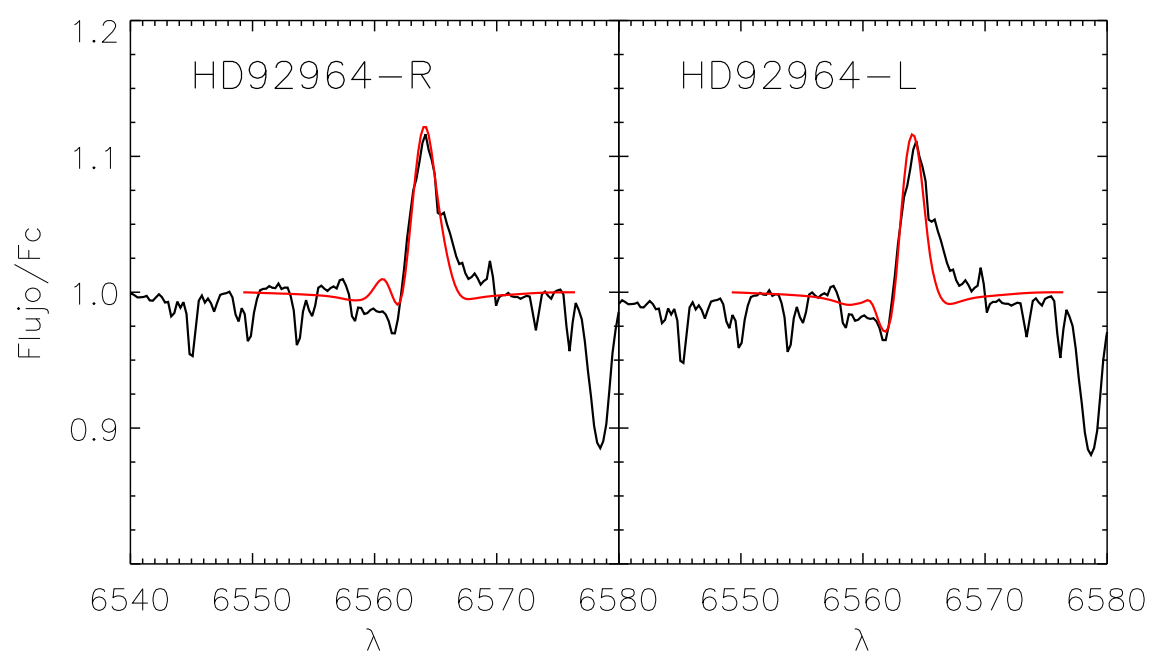

Figura 6.15. Ajustes del perfil de H $\alpha$ para HD 92964.

obtener el ajuste con la ley $\beta$ de velocidades, Lefever y sus colaboradores debieron incluir un factor adicional, desconocido, de ensanchamiento en los perfiles a través de la velocidad de macroturbulencia. Este valor corresponde a $v_{\text {macro }}=60 \mathrm{~km} \mathrm{~s}^{-1}$. En este trabajo, no fue necesario incorporar factores de ensanchamiento adicional, más allá de tener el cuenta un valor de $v \sin i$ que se encuentra próximo a los valores citados por Lefever et al. (2007), como puede verse en la tabla 6.4.

HD 92964: Esta estrella es de clase espectral B2.5 Ia y ha sido modelada por Lefever et al. (2007) con una temperatura efectiva de $18000 \mathrm{~K}$, valor que se adopta en este trabajo. Para la gravedad superficial y el radio estelar también se usan valores similares a los de ese trabajo.

El perfil de $\mathrm{H} \alpha$ es de tipo P Cygni, aunque predomina la emisión y la absorción es muy superficial. Los ajustes realizados con la ley rápida (izquierda) y lenta (derecha), pueden verse en la figura 6.15. El mejor ajuste está dado por la solución lenta, aunque ambos regímenes muestran problemas en el ala roja y no dan cuenta de la extensión completa de la componente en absorción.

Tanto los valores de $\mathrm{v}_{\infty}$ como de $\dot{M}$ son menores que los estimados por Lefever et al. (2007). Puede notarse también que, en el ajuste de este trabajo, fue necesario adicionar una velocidad macroscópica para el ensanchamiento apropiado del perfil. Sin embargo, en los ajustes realizados para ambos regímenes, los valores adoptados aquí son considerablemente menores (siendo el mayor, $18 \mathrm{~km} \mathrm{~s}^{-1}$ ) que los usados $\left(50 \mathrm{~km} \mathrm{~s}^{-1}\right.$ ) por Lefever et al. (2007). Esa diferencia podría deberse a la variabilidad de la estrella.

HD 198478: Esta estrella es de clase espectral B3 Ia, y se encuentra ubicada en la asociación Cyg OB7. Para modelarla se adopta una temperatura efectiva de 18800 $\mathrm{K}$, un valor del logaritmo de la gravedad superficial de 2.43 y un radio de $57 \mathrm{R}_{\odot}$, cantidades muy próximas a los valores medios derivados por Kraus, Haucke, Cidale, Venero, et al. (2015). Se trata de una supergigante B que presenta mucha variabilidad espectrofotométrica (por ejemplo, Maharramov 2013), la cual es atribuida a la presencia 


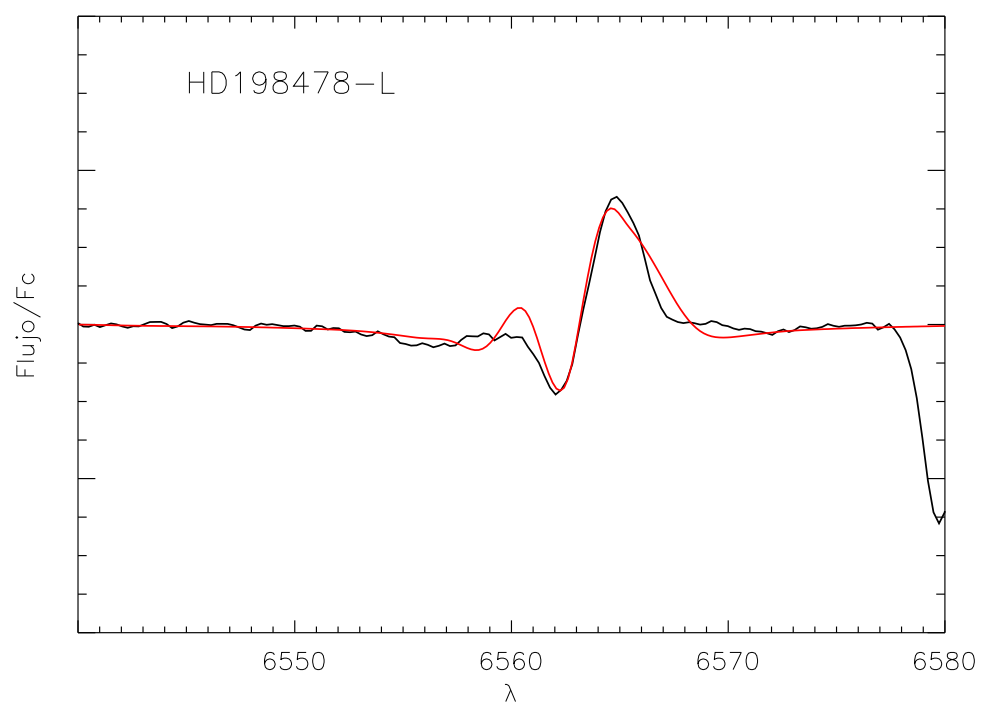

Figura 6.16. Ajuste del perfil de H $\alpha$ para HD 198478.

de oscilaciones no radiales (por ejemplo, Yadav \& Glatzel 2016). Kraus, Haucke, Cidale, Venero, et al. (2015) detectaron la presencia de intensa y rápida variabilidad en los perfiles de línea, los cuales pueden cambiar de noche a noche, y aún en menor período. También derivaron numerosos períodos que sugieren una relación entre la actividad fotosférica y eyección de materia en el viento. A partir de su análisis, Kraus, Haucke, Cidale, Venero, et al. (2015) propusieron que HD 198478 se encuentra en una fase posterior a la de supergigante roja, cruzando el diagrama HR hacia la región azul (ver sección 1.2.2.

El ajuste al perfil observado de $\mathrm{H} \alpha$ es regular, como puede verse en la figura 6.16, y está dado por una solución lenta. Prinja et al. (1990) estimaron una velocidad terminal de $470 \mathrm{~km} \mathrm{~s}^{-1}$ a partir de las líneas de CIV, mientras que Prinja \& Massa (2010) obtuvieron un valor más alto, de $560 \mathrm{~km} \mathrm{~s}^{-1}$ usando Si IV. Ambos valores son demasiado bajos respecto a la predicción teórica de la teoría estándar que sugiere, para este objeto, una velocidad del orden de $1000 \mathrm{~km} \mathrm{~s}^{-1}$ (Lamers et al. 1995). Más aún, Markova \& Puls (2008) sugirieron que el valor de $470 \mathrm{~km} \mathrm{~s}^{-1}$ era demasiado alto para lograr el ajuste de la línea $\mathrm{H} \alpha$, sugiriendo que la velocidad terminal del viento debería ser $200 \mathrm{~km} \mathrm{~s}^{-1}$. Por otra parte, Markova \& Puls (2008) obtuvieron un valor de la tasa de pérdida de masa con mucha incerteza, comprendido entre $0.1175 \times 10^{-6}$ y $0.407 \times 10^{-6}$ $\mathrm{M}_{\odot} \mathrm{año}{ }^{-1}$.

A pesar del ajuste aproximado a la forma del perfil observado de $\mathrm{H} \alpha$, los valores de $\mathrm{v}_{\infty}$ y de $\dot{M}$ obtenidos en este trabajo son muy próximos a los valores medios derivados por Kraus, Haucke, Cidale, Venero, et al. (2015), como puede verse en la tabla 6.4. En este último trabajo, los autores usaron una ley $\beta$ de velocidades con $\beta=2$.

\section{Supergigantes B tardías (B3.5 - B9.5)}

HD 74371: Esta es una estrella de clase espectral B6 Iab/b. Fraser et al. (2010) le asigna una temperatura efectiva de $13400 \mathrm{~K}$ y un valor de $\log \mathrm{g}$ de 1.9 , valores 


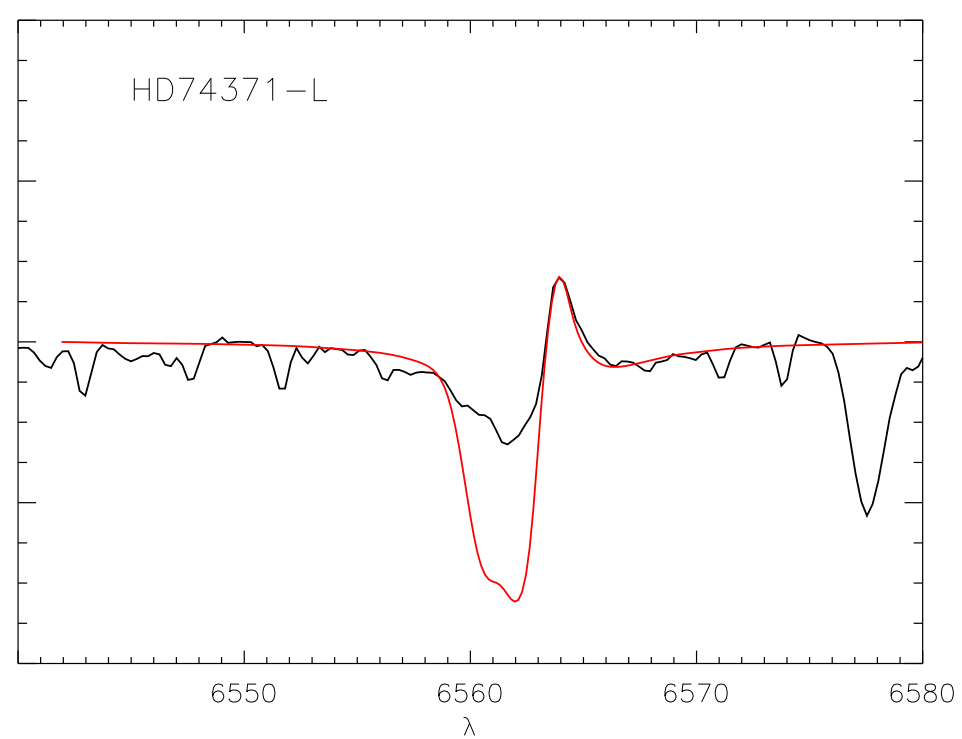

Figura 6.17. Ajuste del perfil de H $\alpha$ para HD 74371.

prácticamente similares a los usados en el modelo de este trabajo.

$\mathrm{El}$ ajuste de $\mathrm{H} \alpha$ puede verse en la figura 6.17. Puede reconocerse que sólo ha podido ser reproducida la componente en emisión. Es muy probable que la escasa profundidad de la componente en absorción, que no ha podido ser ajustada en el perfil, se deba a emisión de relleno generada adicionalmente a lo largo del viento.

No hay datos sobre los parámetros del viento de esta estrella en la literatura. El ajuste del perfil pudo conseguirse con una solución lenta, dando un valor de $\dot{M}$ de $0.24 \times 10^{-6} \mathrm{M}_{\odot}$ año ${ }^{-1}$ y un valor de $\mathrm{v}_{\infty}$ de $182 \mathrm{~km} \mathrm{~s}^{-1}$. Estos valores están en estrecho acuerdo con los valores encontrados por M. Haucke (comunicación privada) mediante la ley $\beta$ de velocidades.

En general, las velocidades terminales encontradas para los ajustes de la línea $\mathrm{H} \alpha$ a las observaciones del grupo de estrellas, son considerablemente más bajas que las medidas en líneas de iones altamente ionizados, tales como el Si IV, C IV o N V en el UV. Resulta llamativo que las velocidades terminales bajas obtenidas, son capaces de reproducir prácticamente todo el perfil de la línea $\mathrm{H} \alpha$. Sería interesante realizar cálculos de perfiles de líneas en el UV, usando las soluciones lentas, de modo de evaluar si estas soluciones son capaces de reproducir los perfiles observados. En este caso, habría una problemática dualidad en los modelos, produciendo cambios profundos en el conocimiento de las condiciones físicas de los vientos de las estrellas supergigantes B.

Otra posibilidad que ha sido planteada en numerosas ocasiones por varios autores, es que el viento de algunas estrellas se encuentre desacoplado entre las especies mayoritarias como el hidrógeno, y las de baja abundancia como los iones de $\mathrm{Si}, \mathrm{C}$ o N (Springmann \& Pauldrach 1992, Krtička \& Kubát 2001). Si el acoplamiento culombiano entre estos iones y el hidrógeno no es efectivo, podrían coexistir dos componentes de viento con diferentes velocidades (ion runaway). 
Con las herramientas de cálculo con las que se cuenta en este trabajo no es posible realizar esos análisis.

\subsubsection{Errores en las estimaciones de los parámetros del viento}

Para estimar los errores en la determinación de los parámetros del viento, a partir de los ajustes de los perfiles de la línea $\mathrm{H} \alpha$, es necesario considerar algunas fuentes de error diferentes.

Por un lado, el error introducido en el uso de datos como la temperatura efectiva, la gravedad superficial o el radio de las estrellas, tomados de la literatura, los cuales tienen sus propias incertezas de origen, sujetas a los métodos bajo los cuales, estos parámetros fundamentales fueron determinados. En el marco conceptual de este trabajo no se busca deducir esa información por medios propios, por lo que son tomados como datos básicos.

La adopción de uno u otro valor de estos parámetros tiene repercusión en los perfiles obtenidos, y por ende, en los ajustes realizados. Por ejemplo, puede notarse que los cambios en la emisión en la línea $\mathrm{H} \alpha$ son perceptibles ante variaciones del orden de $\mathrm{T}_{\text {eff }} \sim 300 \mathrm{~K}$. Respecto a la gravedad superficial, cambios de log g en alrededor de 0.1 dex generan leves variaciones. Los radios estelares también introducen cambios si se los modifica en un rango cercano a $5 \mathrm{R}_{*}$.

El uso de FASTWIND también introduce errores en las determinaciones de los parámetros del viento, a partir de las aproximaciones propias del código, de manera que se trata de errores intrínsecos. Sin embargo, como todos los ajustes son realizados con el mismo código, y bajo las mismas condiciones, es esperable la misma tendencia en todos los espectros sintéticos.

La elección de los parámetros de la fuerza de radiación es muy importante, siendo el parámetro $\alpha$ el que más sensibilidad produce sobre los perfiles. Estas dependencias se describen con detalle en las secciones precedentes. En general, los errores en la tasa de pérdida de masa obtenidas son del orden del 5 al $10 \%$, mientras que los producidos en la velocidad terminal son del orden del $10 \%$.

Habiendo obtenido estos resultados a partir de los ajustes de perfiles de la línea $\mathrm{H} \alpha$, es posible realizar una discusión sobre la capacidad de las soluciones hidrodinámicas estudiadas, para modelar las características globales de los vientos de las estrellas supergigantes B. 


\section{Capítulo 7}

\section{Discusión}

Resumen del capítulo: Se evalúa si los vientos impulsados por radiación en rotación con sus soluciones rápida, $\Omega_{\text {lenta }}$ y $\delta_{\text {lenta }}$ pueden ser capaces de representar las características principales de los vientos de las estrellas supergigantes B. Se analizan las limitaciones de estos modelos impuestas por las principales hipótesis planteadas. En este marco y con la información brindada por el ajuste de las observaciones del capítulo previo, se reconocen las restricciones a los valores de los parámetros para las estrellas supergigantes $B$. Se evalúan, en el nuevo escenario de los régimenes estudiados, conceptos centrales de la teoría de vientos impulsados por radiación tales como la relación entre las velocidades terminales y la de escape o la WLR. Se propone la posibilidad de dar respuesta a la variabilidad de los vientos, en el marco de los modelos propuestos.

\subsection{Validez de las aproximaciones de los modelos}

En los capítulos previos se ha presentado un conjunto de modelos hidrodinámicos basados en hipótesis simples, con la intención de modelar el escenario complejo de los vientos de las estrellas supergigantes B. A continuación se recapitulan las hipótesis requeridas para el desarrollo de los modelos, basados en las soluciones de las ecuaciones hidrodinámicas, y se evalúan las fortalezas y debilidades de las mismas.

Las hipótesis principales usadas en los modelos son:

(a) Geometría: Todos los modelos considerados son solución de las ecuaciones hidrodinámicas en simetría esférica, es decir modelos 1D que sólo dependen de la coordenada radial. El factor preponderante que podría generar un apartamiento considerable de esta geometría simple es, precisamente, la rotación estelar. Sin embargo, las estrellas supergigantes B tempranas tienen una velocidad de rotación del orden de $100 \mathrm{~km} \mathrm{~s}^{-1}$; valor que decrece hacia subtipos más tardíos, alcanzando una velocidad aproximada de 30 o $40 \mathrm{~km} \mathrm{~s}^{-1}$ en el caso de una supergigante A (Kudritzki et al. 1999), como se presenta en la sección 1.2.3. Por lo tanto, es esperable que la aplicación de esta hipótesis para modelar el viento de las estrellas supergigantes B, no tenga consecuencias severas, dado que estos objetos son, en su mayoría, rotadores bajos o intermedios. 
Solamente en el caso de rotadores rápidos $(\Omega \gtrsim 0.8)$, sería más apropiado usar modelos 2D (o 3D) para dar cuenta de las asimetrías producidas por la rotación, tanto en la forma y distribución superficial de la temperatura de la estrella, como en la estructura de densidad en el viento. Tal podría ser el caso de las estrellas supergigantes $\mathrm{B}[\mathrm{e}]$, para las cuales se ha aplicado la solución $\Omega_{\text {lenta }}$, como se menciona en la sección 3.3.3.

Los modelos 2D y 3D también permitirían analizar características de los vientos que no pueden estudiarse con estos modelos 1D simples, por ejemplo, la formación de una estructura discoidal (Bjorkman \& Cassinelli 1993), su inhibición por la presencia de las fuerzas no radiales Owocki et al. (1996), o la formación de vientos prolados en NLTE al considerar oscurecimiento gravitatorio (Petrenz \& Puls 2000). Esos objetivos están fuera de los propósitos de este trabajo.

Por lo tanto, se puede aceptar que el viento de la mayoría de las estrellas supergigantes $\mathrm{B}$, se encuentra en expansión casi esférica y usar modelos $1 \mathrm{D}$, como se hace en la mayoría de los trabajos publicados (por ejemplo Lefever et al. 2007; Markova \& Puls 2008) o como se usa en los códigos destinados para modelarlas (sección 5.2).

Otra de las aproximaciones usadas en este trabajo consiste en que, los modelos dan cuenta de la pérdida de materia en el plano del ecuador estelar (ecuación 3.9), y que se extienden estos valores al resto de la estrella, como representativos de la tasa de pérdida de masa completa de la estrella.

Por considerar simetría esférica, se está suponiendo que la estrella, en todas sus latitudes, produce la misma expulsión de materia que en el ecuador, y que la componente radial obtenida del campo de velocidades es una solución representativa para cualquier latitud.

(b) Corrección por disco finito esférico: Se ha adoptado en este trabajo una corrección por disco finito (sección 2.2.8 considerando simetría esférica y despreciando el oscurecimiento al borde estelar. Nuevamente, como se trata de estrellas con baja rotación, la distorsión en la forma de la estrella debida a la rotación puede despreciarse, frente a otros efectos de mayor peso. Sin embargo, este efecto sería importante para el caso de rotadores rápidos como las supergigantes B[e]. Como consecuencia de la distorsión de la superficie estelar, el achatamiento de la estrella contribuye a generar un viento polar rápido y un viento denso y lento en el plano ecuatorial de la estrella. Algunos cálculos preliminares de los efectos del factor de corrección oblado fueron discutidos por Araya et al. (2011) y por Araya et al. (2017), quienes encuentran que la topología de la ecuación de momento es modificada, y que pueden existir otros puntos críticos. Tales efectos no son tomados en cuenta en este trabajo.

(c) Velocidad térmica constante: Para la resolución de las ecuaciones hidrodinámicas en este trabajo, se supone que el valor de $v_{t h}$ (ecuación 2.32) es constante a lo largo de todo el viento, lo cual es una consecuencia directa de la suposición de vientos isotérmicos y de composición uniforme. Esta es una hipótesis generalizada para la teoría de vientos impulsados por radiación. Se basa en el argumento 
que establece que la contribución de los gradientes de presión, en la ecuación de momento, debidos a los cambios térmicos a lo largo del viento, producen un efecto de segundo orden, comparado con la aceleración radiativa debida a la absorción de momento en las líneas espectrales (Lamers \& Cassinelli 1999).

La suposición de una temperatura constante para todo el viento es una debilidad de estos modelos, ya que las variaciones en la temperatura están fuertemente ligadas a los cambios en los gradientes de ionización. En este trabajo, estos gradientes de ionización son tomados en cuenta, indirectamente, mediante el parámetro $\delta$, como un modo de establecer el régimen del viento. La parametrización de la fuerza de radiación y su cálculo estadístico producen una inconsistencia en los modelos. Sin embargo, puede argumentarse que las fluctuaciones de temperatura, a lo largo del viento, no alterarán suficientemente las soluciones, en tanto los valores adoptados para $\delta$ no fueren excesivamente grandes (por ejemplo, manteniendo $\delta$ $\lesssim 0.4$ ). Tal es el rango de valores de $\delta$ adoptado en este trabajo.

Por otro lado, la constancia de la temperatura a lo largo del viento sólo se aplica en las ecuaciones hidrodinámicas. El transporte de radiación, que se calcula simultáneamente con las ecuaciones de equilibrio estadístico para el estado del material, son obtenidas usando una distribución de temperaturas dada por el balance térmico de los electrones (Kubát et al. 1999). Esta es la distribución de temperaturas implementada en el código FASTWIND (sección 5.2.1).

El rol de altos gradientes de temperatura también puede ser importante en rotadores rápidos, en los que puede esperarse una disminución de temperatura desde los polos hacia el ecuador estelar. Este efecto podría cambiar la estructura de ionización del flujo de materia con la latitud, generando diferentes clases de regímenes de viento, y favoreciendo la formación de estructuras discoidales en expansión o regiones de discontinuidad en las variables físicas. Algunos modelos basados en estas suposiciones fueron discutidos por Zickgraf et al. (1985); Lamers \& Pauldrach (1991); Curé et al. (2005); Araya et al. (2017). En los últimos dos trabajos citados, los autores también analizaron los efectos del salto de bi-estabilidad en temperatura, como un mecanismo capaz de producir un cambio en el régimen de viento a una cierta latitud. Esos modelos están restringidos a rotadores críticos con temperaturas próximas a la del salto de bi-estabilidad (21000 K; Lamers et al. (1995)).

(d) Corrientes meridionales: En este trabajo se desprecian los efectos de las corrientes meridionales entre las regiones polares y las ecuatoriales (von Zeipel 1924). Estos flujos meridionales son más probables en muy altos rotadores, para los cuales se incrementaría la densidad hacia el plano ecuatorial, favoreciendo aún más la formación de estructuras discoidales. Este problema está fuera de los objetivos de este trabajo, y su análisis requeriría, al menos, un tratamiento 2D de las ecuaciones.

(e) Constancia de los parámetros de la fuerza de radiación: Muy pocos trabajos han tenido en cuenta que los parámetros $\alpha, k$ y $\delta$ pueden variar a lo largo del viento. Kudritzki (2002) sugirió una aproximación a este problema pero nunca 
se avanzó en el tratamiento cuantitativo del caso. En esta tesis se consideran a estos parámetros como constantes.

A pesar de las aproximaciones consideradas en los modelos de este trabajo, tales como la adopción de vientos isotermos y con simetría esférica, se puede considerar que las soluciones presentadas en esta tesis son adecuadas para describir las principales características globales de los vientos, tales como las tasas de pérdida de masa o las velocidades terminales para supergigantes B en rotación baja o intermedia. Estas soluciones hidrodinámicas son el punto de partida para el desarrollo de modelos más sofisticados, donde los efectos mencionados anteriormente sean tenidos en cuenta, permitiendo un análisis más detallado de la fenomenología observada en los vientos de estas estrellas.

\subsection{Restricciones a los valores de los parámetros}

De acuerdo a los análisis llevados a cabo en los capítulos 4 y 6 , puede configurarse un rango para los valores de los parámetros de la fuerza de radiación, de modo que los mismos generen modelos hidrodinámicos apropiados para las estrellas supergigantes B. Algunos parámetros que pueden encontrarse en la literatura están tabulados en la tabla 2.1, a modo de comparación.

Los rangos convenientes de los valores de estos parámetros son:

- $\boldsymbol{k}$ : Como se menciona en la sección 2.2.9, los valores que se asignan en la literatura al parámetro $k$, están comprendidos entre 0.15 y 0.5 (tabla 2.1). Algunos valores ligeramente por debajo del límite inferior de ese rango pueden ser conveniente para los modelos presentados en este trabajo. Los mismos pueden dar cuenta de vientos más débiles como los de la estrella HD 52382 (tabla 6.4) para la cual se pudo ajustar el perfil de la línea $\mathrm{H} \alpha$ con valores de $k \sim 0.11$.

Sin embargo, se encuentra que el extremo superior en el rango mencionado arriba para valores de $k$, es extremadamente alto, por lo que es necesario que se lo reconsidere, en su aplicación a las supergigantes B.

Para el régimen rápido, el extremo superior podría considerarse alrededor de valores cercanos a 0.32 . De la figura 4.11 puede verse que, de superar este valor, se obtendrían valores muy altos para la tasa de pérdida de masa. Por otro lado, el mismo gráfico muestra que la falta de vinculación del parámetro $k$ con la velocidad terminal, hace que sea posible tener un amplio rango de valores para $\mathrm{v}_{\infty}$, a partir de un mismo valor de $k$, modificando el parámetro $\delta$.

Por la misma razón, en el régimen lento, los valores de $k$ deberían ser un poco más bajos que para el régimen rápido. La figura 4.11 muestra que $k$ no debería superar un valor próximo a 0.28 . Se ha encontrado además, que valores tan elevados de $k$ darían emisiones elevadas, lo cual no está presente en los perfiles observados de $\mathrm{H} \alpha$. Entonces, en este caso, es conveniente considerar, para este parámetro, valores cercanos o menores a 0.22 , como puede sugerirse a partir de la figura 6.5 . Vale mencionar que, para los ajustes de los perfiles observados de $\mathrm{H} \alpha$ dados en la sección 6.4. el valor máximo de $k$ que se empleó fue de 0.23. 
El requerimiento de valores bajos de $k$ podría estar asociado, además, al fenómeno del clumping, que no se incorpora en este trabajo.

Se debe tener en cuenta, además, que estas estimaciones están basadas en la suposición de que las estrellas son simples y no tienen contribuciones adicionales de flujo externos. Particularmente, los perfiles podrían verse diluidos por binariedad, lo cual requeriría un replanteo de los valores apropiados del parámetro $k$, permitiendo valores mayores a los mencionados en los párrafos previos.

- $\boldsymbol{\alpha}$ : La mayoría de los estudios sobre estrellas tempranas adoptan valores para el parámetro $\alpha$ comprendidos entre 0.5 y 0.6. Como una cota mínima Martins et al. (2005) ha sugerido que un valor de $\alpha \sim 0.3$ es necesario para obtener consistentemente las bajas velocidades terminales y tasas de pérdida de masa en algunas estrellas $\mathrm{O}$ enanas, en el problema aún no resuelto de los vientos débiles (weak winds, sección 2.6.5).

Los modelos calculados en este trabajo que permiten obtener valores de los parámetros del viento más acordes a los observados en las supergigantes $\mathrm{B}$, indican que $\alpha$ debería mantenerse, preferentemente, en valores en el rango comprendido entre 0.45 y 0.55 . Para el régimen rápido, la figura 4.12 muestra que ese rango podría extenderse hasta $\sim 0.6$, siempre que $\delta$ no fuera superior a 0.15 , simultáneamente. Este resultado está en concordancia con los usados comúnmente en la teoría estándar de vientos impulsados por radiación.

Para el régimen lento $\alpha$ debería mantenerse mucho más acotado, sin posibilidades de exceder el valor 0.55 . De otra manera, las tasas de pérdida de masa (determinados a partir de los perfiles sintéticos resultantes, los cuales tendrían mucha emisión) se harían demasiado grandes como para ajustar las observaciones de las supergigantes B. Por otra parte, los valores de $\alpha$ por debajo de 0.45 dan lugar a perfiles de $\mathrm{H} \alpha$ completamente en absorción (figura 6.6). Si bien hay muchas estrellas supergigantes B con perfiles en absorción, resulta más apropiado modelar las líneas en emisión para evitar grandes incertezas en la determinación de los parámetros del viento.

La tabla 6.4 muestra que la mayoría de las supergigantes B observadas puede ser modeladas con valores ubicados en un estrecho margen alrededor de $\alpha=0.5$.

- $\delta$ : En este trabajo, el valor del parámetro $\delta$ es decisivo, ya que determina el tipo de régimen, rápido o lento, imperante en el viento. Los modelos con $\delta$ pequeños corresponden al primer régimen, mientras que los mayores al segundo. El valor medio de $\delta$ para el cual se produce el cambio entre uno y otro régimen, está determinado por la velocidad de rotación de la estrella, que localiza a la brecha.

Puede notarse en la figura 4.2 que, dado que la mayoría de las supergigantes B son rotadores lentos o intermedios $(\Omega<0.4)$, el régimen rápido domina para valores de $\delta \lesssim 0.20$, mientras que el régimen lento se establece para valores de $\delta \gtrsim 0.3$. Los valores intermedios de $\delta$ en ese rango, generan soluciones no estacionarias, cuya ley de velocidades muestra un gradiente discontinuo (kink o plateau), como puede verse en la figura 4.15. Estas soluciones deberán ser analizadas con más profundidad en futuros estudios. 
Por otro lado, $\delta$ no debe superar el valor de 0.4 , a menos que las emisiones de los perfiles observados sean muy elevadas, lo que no se encuentra en la muestra analizada, ni es común en esta clase de estrellas. Valores tan altos de $\delta$ sólo pueden ser combinados con valores bajos de $k$ (como se hace para el ajuste de HD41 117 en la sección 6.4.3), de otra manera las emisiones de los perfiles P Cygni de la línea $\mathrm{H} \alpha$ se hacen muy intensas, como se observa en la figura 6.1

El valor de $\delta$ también puede afectar el aspecto de los perfiles de $\mathrm{H} \alpha$, incorporando estructuras más complejas en su componente en absorción. Eso puede suceder, para el régimen rápido, cuando $\delta$ ubica al modelo muy cerca de la brecha (sección 6.3.2).

\subsection{Características físicas de las soluciones lentas}

Dado que la solución $\delta_{\text {lenta }}$ ha sido poco explorada, en esta sección se estudian algunas de las relaciones más importantes dadas por la teoría de vientos impulsados por radiación aplicadas, en este caso, a este régimen particular. El propósito es evaluar si esas relaciones se siguen manteniendo bajo un régimen de viento diferente del que surge de la teoría estándar. Si estas relaciones no son satisfechas en el régimen lento y algunas supergigantes B tienen vientos con las características propias de esta clase de solución, el ajuste de sus espectros por medio de las soluciones estándar introducirá errores significativos en las medidas derivadas.

La evaluación de estas relaciones para el régimen lento se realiza, en algunos casos, por medio del análisis de las predicciones de los resultados teóricos puros y, en otros, por medio de la comparación de la teoría con los valores obtenidos en el ajuste de los perfiles de la línea $\mathrm{H} \alpha$ presentados en la sección 6.4 o de datos observacionales publicados por otros autores.

\subsection{1. $\quad$ Las soluciones lentas y sus orígenes}

Las soluciones $\Omega \delta_{\text {lentas }}$ o lentas en general, constituyen escenarios alternativos a los propuestos por la teoría estándar, que pueden proporcionar nuevos modos de modelar los vientos impulsados por radiación en estrellas masivas, permitiendo avanzar en la comprensión de sus características físicas. Es importante remarcar que, tanto la soluciones rápidas como las lentas, son soluciones de un mismo conjunto de ecuaciones hidrodinámicas, en las cuales tienen diferente peso los términos que las componen, dando origen a los regímenes estudiados.

En el caso de rotadores rápidos $(\Omega \geq 0.8)$, la solución de las ecuaciones hidrodinámicas es del tipo $\Omega_{\text {lenta }}$ ya que el término de aceleración centrífuga de las ecuaciones hidrodinámicas se vuelve dominante, reduciendo considerablemente la gravedad efectiva y desplazando el punto crítico en la dirección del flujo, aproximándolo hacia la posición del punto sónico CAK (Curé 2004). Como consecuencia, se establece en el viento un gradiente de velocidades más bajo en la región subsónica.

En el dominio de los rotadores lentos, el término de la fuerza de radiación, expresado a través de la parametrización de Abbott (1982b) dada por la ecuación 2.64, tiene un factor dependiente de la profundidad óptica $\tau^{-\alpha}$ que domina la ecuación de momento. 
La presencia de gradientes de ionización más pronunciados (expresados por un factor de la forma $\left(n_{e} / W\right)^{\delta}$ en la ecuación 3.9 introduce una dependencia adicional con la densidad. El efecto total de ambas contribuciones puede resumirse sustituyendo el parámetro $\alpha$ por $\alpha^{\prime}=\alpha-\delta($ Kudritzki et al. 1999). A mayor valor de $\delta$, resulta menor el valor de $\alpha^{\prime}$, con el consecuente incremento en $\dot{M}$ (como se cumple, por ejemplo, en la ecuación 2.91 y el decremento en la velocidad del viento. Esta clase de solución es el régimen $\delta_{\text {lento }}$, encontrado por Curé et al. (2011).

\subsubsection{Eficiencia en la transferencia de momento del campo de radiación}

El hecho de que la solución lenta origine tasas de pérdida de masa y velocidades terminales diferentes para el viento, respecto a los valores suministrados en la solución estándar, sugiere que la transferencia de momento del campo de radiación al material está actuando de una manera diferente.

La variación inversa de $\dot{M}$ y $v_{\infty}$ con respecto a $\delta$ observada en los modelos presentados, por ejemplo, los mostrados en la figura 4.2 , se debe a que el producto $\dot{M} v_{\infty}$ (ecuación 2.107) es proporcional al momento total entregado por el campo de radiación $L / c$. El valor del producto $\dot{M} v_{\infty}$ mide la pérdida de momento del campo de radiación transferido al viento. La teoría de vientos impulsados por radiación predice una proporción entre ambas cantidades, que debe mantenerse casi constante (Abbott 1980).

En la figura 7.1 se grafica el cociente entre $\dot{M} v_{\infty}$ y $L / c$ para verificar este comportamiento en el caso del modelo base T19, en función del parámetro $\delta$ y para diferentes tasas rotacionales $\Omega$. Efectivamente, en el gráfico se puede observar que, en el dominio de la solución rápida (ramas ubicadas a la izquierda de la figura) el cociente se mantiene casi constante, en concordancia con lo predicho por la teoría.

Esta relación se mantiene aún para rotadores rápidos, siempre que se consideren valores bajos de $\delta$.

Sin embargo, en el dominio de la solución $\delta_{\text {lenta }}$ (para altos valores de $\delta$ ), la pérdida de momento se incrementa fuertemente al crecer $\delta$.

Esto significa que, en el régimen $\delta_{\text {lento }}$, la transferencia de momento al viento debe darse de modo mucho más eficiente. Ese aumento en la pérdida de momento del campo de radiación se debe a que, al aumentar la ionización, la abundancia de iones que impulsan al viento decrece mucho más rápidamente con la distancia, que en el caso de un viento de valores pequeños de $\delta$. Por lo tanto, en distancias más cortas, el viento debe ser capaz de absorber mayor cantidad de momento, incrementando la eficiencia de transferencia de momento.

Simultáneamente, el fuerte crecimiento de $\dot{M} v_{\infty}$ respecto a $L / c$ indica que debe haber una deposición adicional de energía/momento en el viento, por encima de la provista por la luminosidad de la estrella. Esto muestra que debe haber una fuente adicional de energía para justificar el estado de alta ionización que supone un parámetro $\delta$ elevado. Esta energía/momento adicional en el viento podría provenir, por ejemplo, de ondas de choque producidas por la inestabilidad del viento (sección 2.6.1). 


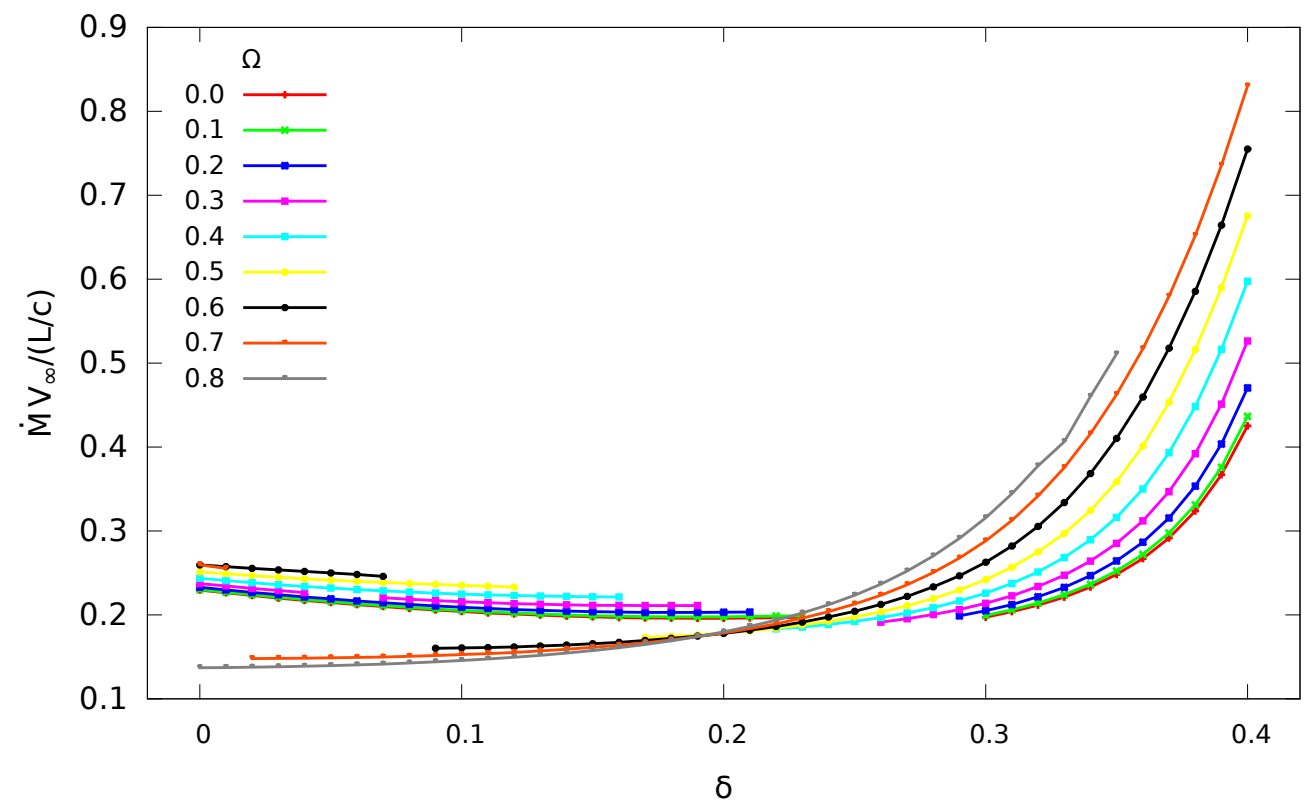

Figura 7.1. Cociente entre la pérdida de momento del viento y la pérdida de momento del campo de radiación vs. $\delta$ para el modelo base T19. El momento transferido se vuelve más eficiente en el dominio de la solucion lenta.

\subsubsection{Parámetros derivados a partir de las mediciones en las estrellas observadas}

Para poder evaluar la vigencia de las relaciones predichas por la teoría de vientos impulsados por radiación para la solución $\delta_{\text {lenta }}$, se reunen, en la tabla 7.1, algunas cantidades derivadas para las estrellas para las cuales se han ajustado perfiles obtenidos con la solución $\delta_{\text {lenta }}$.

Las columnas de la tabla 7.1 corresponden a: 1) el nombre de la estrella, 2) la velocidad de escape $\mathrm{v}_{\mathrm{esc}}$, calculada de acuerdo a la ecuación 2.88, 3) el coeficiente $\Gamma$, dado por la ecuación 2.25, 4) el logaritmo de la cantidad $\mathrm{D}_{\text {mom }}$ obtenida a partir de la ecuación 2.106 y 5) el logaritmo del invariante Q, de la ecuación 1.1. Las secciones que siguen se referirán a estas cantidades y su valor para el análisis.

\subsubsection{Relación entre $\boldsymbol{v}_{\infty}$ y $\boldsymbol{v}_{\mathrm{esc}}$}

En general, se suele modelar a la mayoría de las supergigantes $\mathrm{O}$ y $\mathrm{B}$ tempranas por medio de una ley $\beta$ de velocidades (ecuación 2.2 con $\beta<1$. Sin embargo, como se mencionó en la sección 2.6, las supergigantes B tardías junto a las supergigantes A exhiben apartamientos notorios de estos ajustes, requiriendo, a menudo, valores de $\beta \geq$ 2. La diferencia en el comportamiento entre ambos grupos de supergigantes puede verse claramente en la relación empírica $v_{\infty} / v_{\text {esc }}$ versus $v_{\text {esc }}$, que se presenta en la figura 7.2 . Los puntos del gráfico incluyen diferentes símbolos correspondientes a las observaciones de Prinja et al. (1990); Lamers et al. (1995); Achmad et al. (1997); Verdugo et al. (1998). Estos valores, tomados de la literatura corresponden a supergigantes B (cruces rosas) 


\begin{tabular}{lcccc}
\hline \hline Estrella & $\mathrm{v}_{\text {esc }}\left[\mathrm{km} \mathrm{s}^{-1}\right]$ & $\Gamma$ & $\log \mathrm{D}_{\text {mom }}$ & $\log \mathrm{Q}$ \\
\hline HD 41 117 & 345.5 & 0.382 & 27.65 & -12.57 \\
HD 42 087 & 361.8 & 0.196 & 27.09 & -12.71 \\
HD 47 240 & 303.7 & 0.341 & 27.07 & -12.77 \\
HD 52 382 & 239.2 & 0.350 & 26.51 & -12.48 \\
HD 74 371 & 241.3 & 0.247 & 27.36 & -12.78 \\
HD 75 149 & 202.2 & 0.346 & 26.66 & -12.76 \\
HD 92 964 & 206.4 & 0.493 & 26.89 & -12.71 \\
HD 99 953 & 364.9 & 0.244 & 27.50 & -12.57 \\
HD 198478 & 393.6 & 0.275 & 27.83 & -12.62 \\
\hline
\end{tabular}

Tabla 7.1. Cantides derivadas de los modelos de las estrellas supergiantes B.

y a supergigantes A ("X" verdes). Resulta claro que hay dos grupos de estrellas que se ubican en torno a dos relaciones funcionales con pendientes y distribuciones diferentes.

En esta figura, las barras verticales rojas graficadas para diferentes modelos base, representan el rango de valores de $v_{\infty} / v_{\text {esc }}$ correspondientes a todos los modelos con solución rápida obtenidos para diferentes valores de $\delta$ y $\Omega=0.2$. De la misma manera, las barras verticales azules representan los modelos obtenidos para el régimen $\delta_{\text {lento }}$.

La solución rápida ajusta razonablemente bien las velocidades terminales medidas para las estrellas supergigantes B tempranas e intermedias. En cambio, las barras verticales azules, que corresponden a la solución lenta, se adecuan mejor a las supergigantes A tempranas y, posiblemente, a algunas B tardías.

Los triángulos grises y los rojos corresponden a los valores dados por los modelos para algunas supergigantes B, obtenidos por Haucke, Araya, Arcos, Curé, Cidale, Kanaan, Venero, \& Kraus (2015), y a los medidos en este trabajo, respectivamente. Los últimos valores corresponden a los presentados en la tabla 7.1 .

Dada la ubicación de los puntos correspondientes a la solución $\delta_{\text {lenta }}$, puede observarse que los valores de $v_{\infty} \mathrm{y}$, por ende, los parámetros de la fuerza de radiación utilizados, son más adecuados para representar a las supergigantes B tardías y las supergigantes A. Este último resultado está de acuerdo a lo encontrado por Curé et al. (2011).

\subsubsection{La relación momento del viento - luminosidad para el régimen lento}

La relación Momento del Viento - Luminosidad (WLR) para el viento de las estrellas tempranas, fue presentada en la sección 2.5.1. Dada la importancia de esta relación para la determinación de distancias espectroscópicas a estrellas masivas galácticas y extragalácticas, resulta de gran interés examinar los resultados para la solución $\delta_{\text {lenta }}$.

Los valores para el logaritmo de $\mathrm{D}_{\text {mom }}$ (ecuación 2.106) en función del logaritmo de $\mathrm{L}_{*} / \mathrm{L}_{\odot}$, obtenidos para la muestra de estrellas modeladas en la sección 6.4 pueden verse distribuidos en la figura 7.3. Se incluyen además los ajustes de esta relación, realizados por Kudritzki \& Puls (2000), para las supergigantes B tempranas e intermedias y las supergigantes A. Los datos para esos ajustes se transcriben en la tabla 2.3 . 


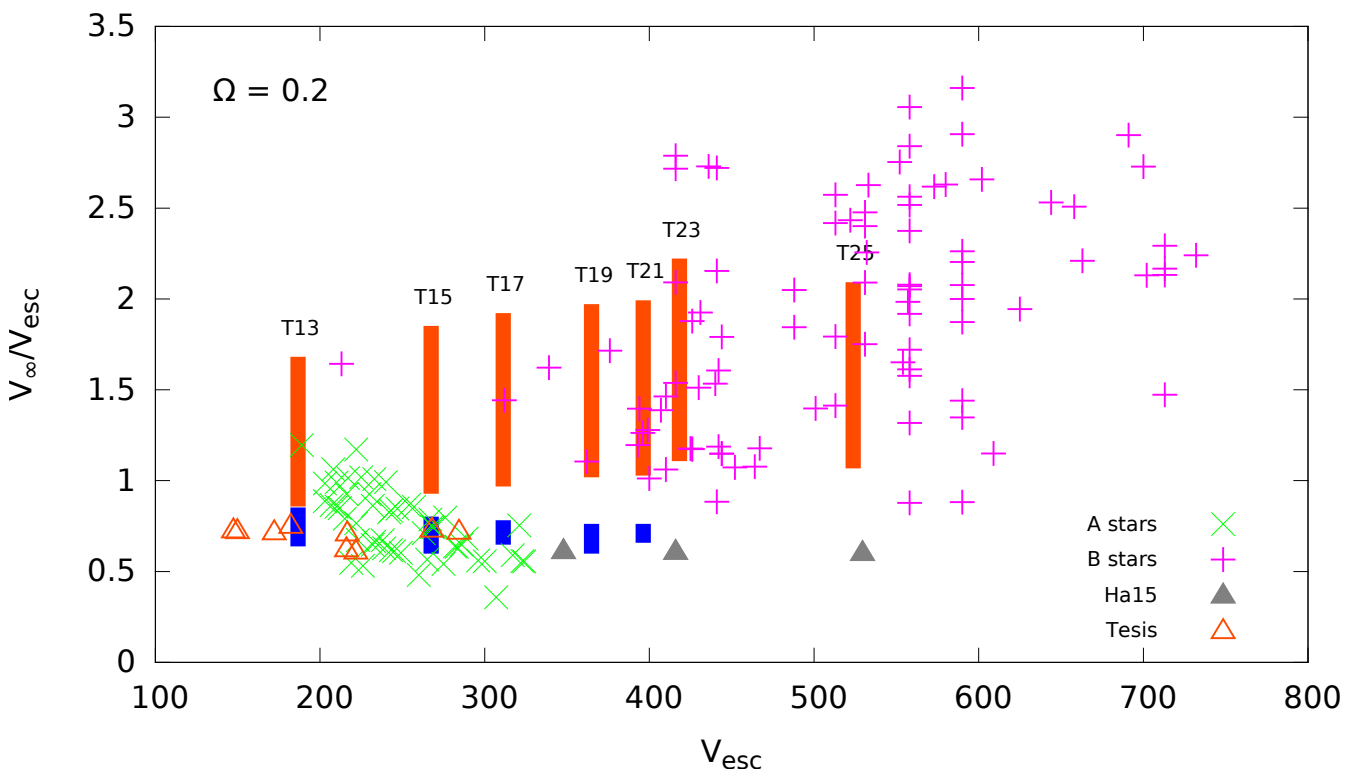

Figura 7.2. Relación entre $v_{\infty} / v_{\text {esc }}$ y $v_{\text {esc }}$ en la que se comparan los resultados de los modelos con mediciones de observaciones. Las barras verticales rojas corresponden al rango completo de soluciones rápida para los modelos base rotulados y calculados para el rango completo considerado de valores de $\delta$. Las barras verticales azules representan el rango de $v_{\infty} / v_{\text {esc }}$ dado para los modelos base en el régimen $\delta_{\text {lento }}$. Estas barras azules podrían extenderse aún más hacia valores más bajos de $v_{\infty} / v_{\text {esc }}$ que los graficados en esta figura, debido a que se ha impuesto un valor límite al parámetro $\delta(0.40)$. Todos los modelos fueron calculados $k=0.32$ y con $\Omega=0.2$, como tasa rotacional representativa de las supergigantes B. Los demás parámetros $(k$ y $\alpha)$ son los presentados en la tabla 4.3. Los triángulos grises corresponden a los valores obtenidos en el trabajo de Haucke et al. (2015), mientras que los triángulos rojos, corresponden a los valores derivados de los modelos ajustados para esta tesis.

La mayoría de las estrellas que han sido ajustadas con el régimen lento en este trabajo, se distribuyen, aproximadamente, en una relación de carácter lineal. Un ajuste por mínimos cuadrados da una relación de la forma:

$$
\log D_{\mathrm{mom}}=1.3723 \log \left(L_{*} / L_{\odot}\right)+19.9643 .
$$

La pendiente resultante de este ajuste "lineal", que se presenta en color rosa en la figura 7.3, está próxima a la obtenida por Kudritzki \& Puls (2000) para las supergigantes $\mathrm{B}$ tempranas, siendo su valor de 1.34 (tabla 2.3). Sin embargo, la ordenada al origen es considerablemente más baja (21.24).

Por otro lado, si se eliminan algunas de las estrellas que presentan mayor dispersión (como HD 92964), resulta que los puntos caen con mayor proximidad a la relación dada para las supergigantes B intermedias.

Efectivamente, los valores de $\log \left(\mathrm{D}_{\mathrm{mom}}\right)$ obtenidos en esta tesis a partir de la solución $\delta_{\text {lenta }}$, coinciden con los encontrados por Kudritzki et al. (1999) y Markova \& Puls 


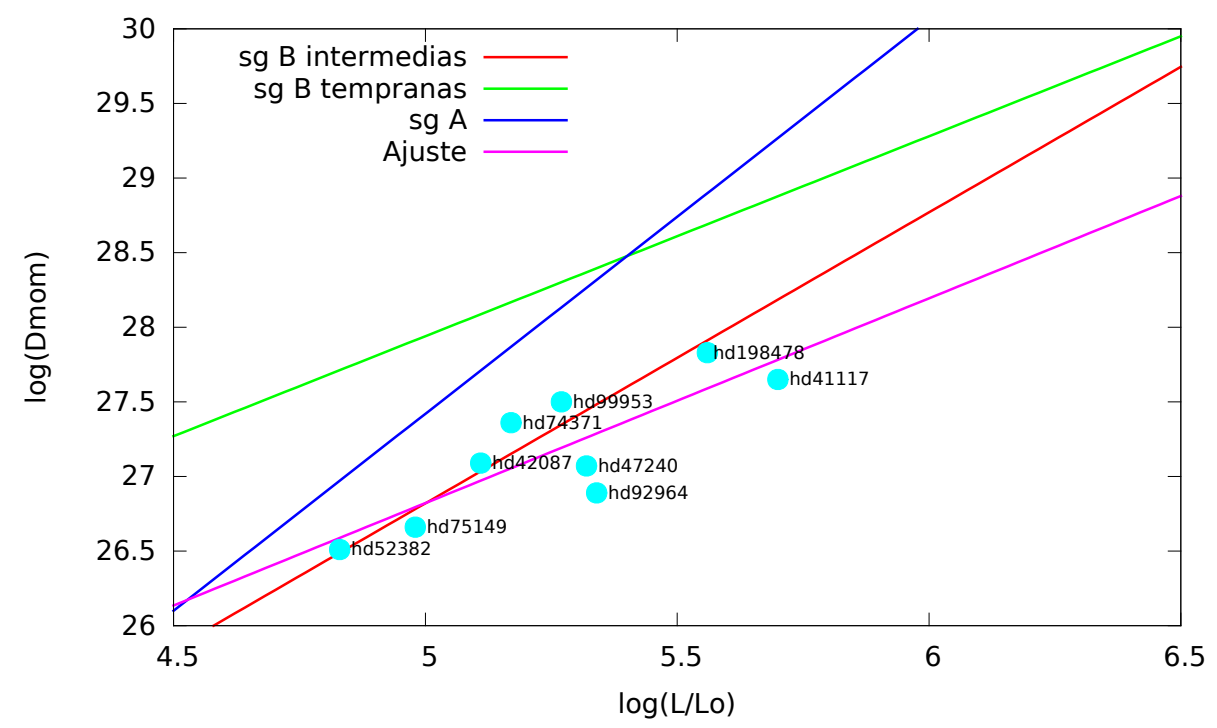

Figura 7.3. Localización de las estrellas modeladas con la solución $\delta_{\text {lenta }}$ respecto a la relación Momento del Viento - Luminosidad para diferentes clases de estrellas dadas por Kudritzki \& Puls (2000). Se incluye el ajuste basado en las estimaciones realizadas en este trabajo, por medio de la recta de color rosa.

(2008) para las supergigantes B intermedias, como puede verse en la figura 7.4. Tanto las mediciones realizadas en esos trabajos como los valores obtenidos en esta tesis, caen por debajo de la predicción teórica (recta anaranjada) dada por Vink et al. (2000). El ajuste lineal a los valores calculados en esta tesis se indican con la línea rosa.

Estos resultados implican que, si las distancias a supergigantes B son derivadas a partir de una relación WLR, pero las estrellas de la muestra tienen sus vientos dominados por el régimen lento, los errores en la determinación podrían ser significativos. Esto sugiere que es necesario realizar una detallada calibración de esta relación para la solución $\delta_{\text {lenta }}$.

\subsection{6. $\quad$ El invariante $\mathrm{Q}$ para el régimen lento}

En el trabajo de Puls et al. (1996) y, especialmente, en el de Puls $(2008)$, se introducen los factores $Q$ y $Q_{\text {res }}$ (ecuación 1.1), llamados invariantes de profundidad óptica. Como se detalla en la sección 1.2.4 las combinaciones de diferentes tasas de pérdida de masa, radios y velocidades terminales, generan modelos que producen las mismas características espectrales, es decir, perfiles de línea con los mismos anchos equivalentes y, en gran medida, aspecto similar.

Dado que estas relaciones han sido obtenidas a partir de un campo de velocidades dado por la ley $\beta$, resulta de interés comprobar si los perfiles de línea conservan su forma y ancho equivalente para la solución $\delta_{\text {lenta }}$. De los dos invariantes, se puede probar el invariante $Q$ :

$$
Q=\frac{\dot{M}}{\left(R_{*} v_{\infty}\right)^{1.5}},
$$




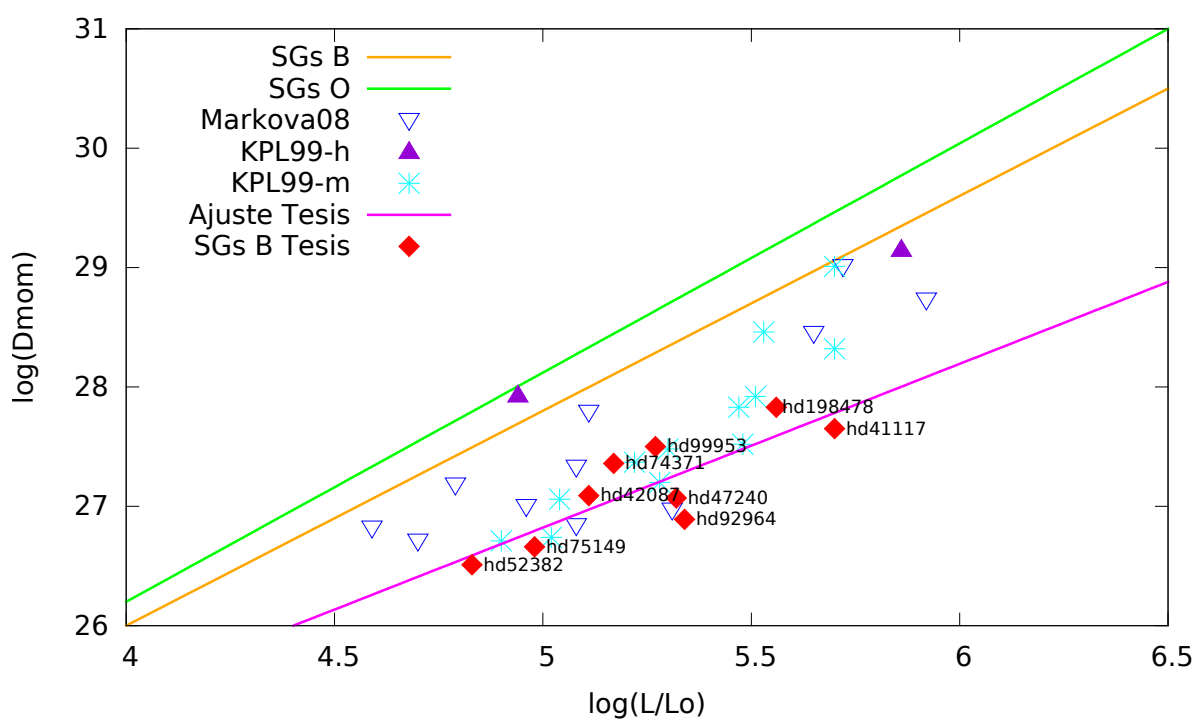

Figura 7.4. Comparación entre la WLR teórica y algunos valores obtenidos a partir de las observaciones. Con las líneas verde y anaranjada se indican las relaciones teóricas dadas por Vink et al. (2000), para supergigantes O y B, respectivamente. Se incluyen los valores obtenidos por Kudritzki et al. (1999) para supergigantes B tempranas (indicadas como KPL99-h) y las intermedias (KPL99-m), junto con los calculados por Markova $\&$ Puls (2008). En diamantes rojos, se muestran los datos obtenidos en esta tesis y el ajuste por mínimos cuadrados (línea rosa).

que corresponde a las líneas originadas en los procesos de recombinación (con opacidad proporcional a $\rho^{2}$ ), como es el caso de la línea $\mathrm{H} \alpha$. Los valores de $Q$ obtenidos para la muestra observacional de estrellas ajustadas se encuentra en la tabla 7.1.

En este caso, para probar la invariancia de $Q$, se calcula con el código HYDWIND un modelo dado, con $k, \alpha$ y $\delta$ fijos, y para un radio $R_{*}$ determinado. Luego se calcula el valor de $Q$ para ese radio, con la fórmula citada. A continuación, se procede a cambiar el radio, por ejemplo, en saltos de $10 R_{\odot}$, y obtener los correspondientes conjuntos de valores distintos de $\dot{M}$ y $v_{\infty}$ que generaran valores diferentes de $Q$.

En este caso, a diferencia del modelo estándar en el cual la forma de la ley de velocidades está predeterminada, no es posible regular $\dot{M}$ y $v_{\infty}$ a priori, para recuperar el valor de $Q$ inicial a partir de otro radio estelar. Para dar con las soluciones hidrodinámicas numéricas apropiadas la única opción, en este trabajo, consiste en modificar los parámetros $k, \alpha$ o $\delta$, uno por vez, hasta recuperar el valor de $Q$. La elección con que se haga el cálculo para mantener constante a $Q$ no es única, ya que las relaciones son fuertemente no lineales.

Los valores elegidos para modelar la estrella en estas pruebas son:

$$
\begin{gathered}
\mathrm{T}_{\text {eff }}=19000 \mathrm{~K} . \\
\log \mathrm{g}=2.5 . \\
\Omega=0.0 . \\
R_{*}=20,30,40,50,60 \text { y } 70 R_{\odot} . \\
\text { Parámetros } \alpha \text { y } \delta \text { fijos. }
\end{gathered}
$$


En esta ocasión, se opta modificar el valor del parámetro $k$. La tabla 7.2 muestra los reajustes efectuados en el parámetro $k$ para recuperar el mismo valor de $Q$, al cambiar el radio estelar:

\begin{tabular}{lccccccc}
\hline \hline Modelo & $k$ & $\alpha$ & $\delta$ & $\dot{M}$ & $v_{\infty}$ & $R_{*}$ & $\log Q$ \\
\hline a20 & 0.167 & 0.500 & 0.34 & 0.0419 & 182.9674 & 20 & -12.72 \\
a30 & 0.186 & 0.500 & 0.34 & 0.1020 & 219.5766 & 30 & -12.72 \\
a40 & 0.200 & 0.500 & 0.34 & 0.1924 & 250.0951 & 40 & -12.72 \\
a50 & 0.211 & 0.500 & 0.34 & 0.3129 & 276.8614 & 50 & -12.72 \\
a60 & 0.220 & 0.500 & 0.34 & 0.4626 & 301.0191 & 60 & -12.72 \\
a70 & 0.229 & 0.500 & 0.34 & 0.6653 & 323.2318 & 70 & -12.71 \\
\hline
\end{tabular}

Tabla 7.2. Tabla de valores de los parámetros del viento que mantienen constante el valor de $\log Q$. Las tasas de pérdida de masa tabuladas están en unidades de $10^{-6}$ $\mathrm{M}_{\odot}$ año ${ }^{-1}$.

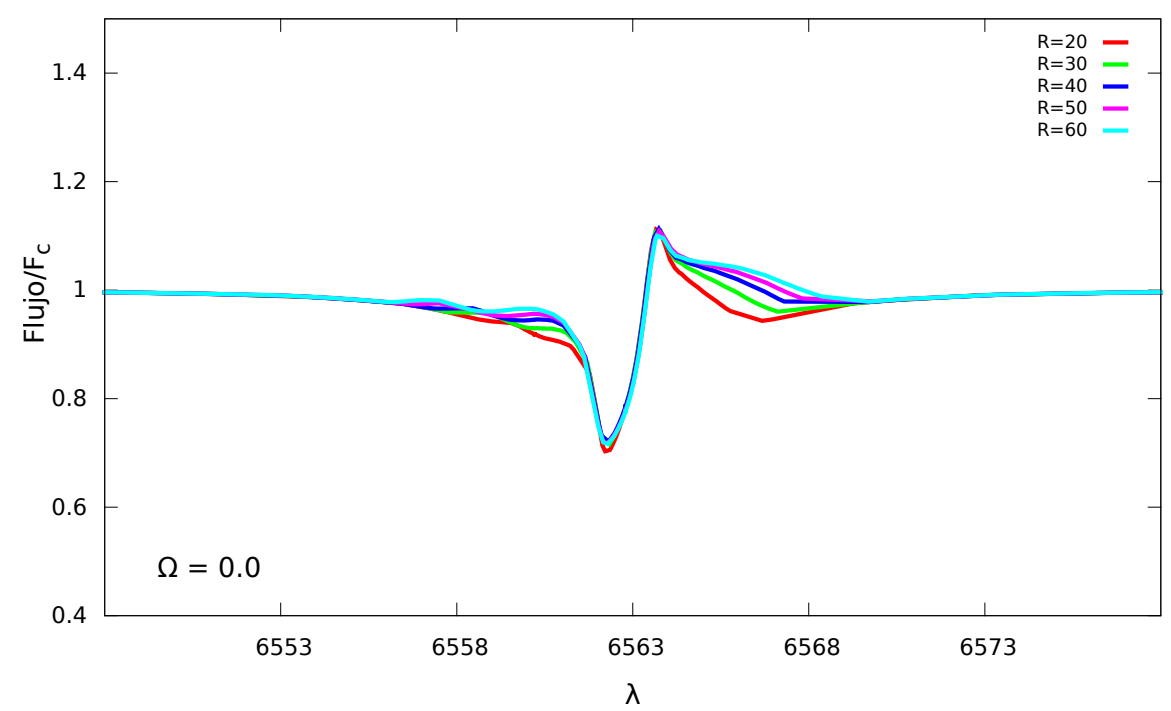

Figura 7.5. Variación de los perfiles teóricos de $\mathrm{H} \alpha$ ante cambios de $\mathrm{R}_{*}, \dot{M}$ y $\mathrm{v}_{\infty}$ que mantienen constante el valor de $Q$.

Los perfiles de $\mathrm{H} \alpha$ resultantes para este conjunto de modelos se muestran en la figura 7.5. Puede notarse que, ante la constancia del parámetro $Q$ para la solución $\delta_{\text {lenta, }}$ el núcleo de la línea se modifica muy poco. Sin embargo, las alas son diferentes para la secuencia de modelos.

Tanto en los modelos presentados y como en otros ensayos (por ejemplo, en los que se modifica $\alpha$ dejando constantes $\delta$ y $k$ ), muestran que el factor $Q$ no es un invariante para el régimen $\delta_{\text {lento }}$. Estas diferencias se acentúan aún más, cuando los valores medios de $k$ empleados son mayores $(k \sim 0.3)$. 


\subsection{Identificación del régimen de viento a partir de los perfiles de línea}

Para evaluar si los regímenes rápido y lento son apropiados para describir el viento de las estrellas supergigantes B, es necesario identificar aquellas características espectrales resultantes que puedan diferenciar a ambos regímenes y ser advertidas en las observaciones. La gran variedad de perfiles de la línea $\mathrm{H} \alpha$ observada en una pequeña muestra como la presentada en la sección 6.4 no hace que ésta sea una tarea sencilla.

Por esa razón, también es importante estimar en qué medida estas peculiaridades espectrales, que pudieran ser identificadas como características propias de un régimen de viento dado, son intensificadas o condicionadas por los parámetros de la fuerza de radiación que definen a cada régimen.

Algunas características de los perfiles pueden dar indicios para reconocer el régimen de viento dominante. Mapeos de los perfiles sintéticos como los mostrados por la figura 6.1 pueden ayudar a alcanzar este objetivo.

Por ejemplo, considerando modelos con un nivel bajo y fijo de ionización ( $\delta$ entre 0.0 y 0.2 ), hay algunas diferencias perceptibles entre los modelos de cada régimen. Los perfiles que fueron generados en el dominio $\delta_{\text {lento }}$ presentan componentes en absorción un poco más profundas, junto a componentes en emisión intensas y anchas en los perfiles $\mathrm{P}$ Cygni. En cambio, los perfiles $\mathrm{P}$ Cygni generados en el régimen rápido son más susceptibles a tener sus componentes en absorción con más "relleno" de emisión.

Para modelos con alto valor de $k$ (cercano a 0.3 ) en el régimen rápido (como puede verse en el panel de abajo a la izquierda en la figura 6.3), dos clases de perfiles P Cygni pueden ser producidos para un mismo régimen, en distintas condiciones de ionización. Estos perfiles son: un perfil P Cygni poco extendido, para valores de $\delta$ cercanos a cero, y un perfil P Cygni muy extenso, cuando los valores de $\delta$ lo ubican cerca de la brecha. Por lo tanto, el ala azul de la componente en absorción del perfil P Cygni podría dar una referencia del valor de $\delta$ e indicar cuán cercano está el régimen respecto a la brecha, si el viento se extiende a longitudes de onda muy lejanas a la de reposo. Esta doble identidad de los perfiles $\mathrm{P}$ Cygni sólo ocurre en el régimen rápido.

Otra diferencia entre los perfiles de regímenes distintos es que las componentes en emisión de los perfiles $\mathrm{P}$ Cygni del régimen rápido, son un poco más pronunciadas o "agudas" en su parte superior, mientras que las emisiones en el régimen lento son más redondeadas. Estas diferencias provienen de la forma del campo de velocidades para cada caso.

También es interesante considerar el caso de una secuencia de modelos que mantienen una tasa de pérdida de masa casi constante, bajo cambios en las condiciones de ionización. Por ejemplo, la primera columna de paneles de la figura 6.1 muestra un conjunto de modelos con valores constantes de $k, \Omega, \alpha$, y casi constante de $\dot{M}$ (de acuerdo a la figura 4.10), pero con cambios en $\delta$. En el régimen rápido se pueden encontrar variaciones muy pequeñas en esta secuencia. En cambio, al pasar al otro régimen, el perfil ya no es del tipo P Cygni, sino que se transforma en un perfil en absorción pura. Entonces, a un mismo valor de la tasa de pérdida de masa, un régimen generaría perfiles P Cygni poco intensos, mientras que el otro, perfiles en absorción.

En base a estos indicios, a partir de los perfiles observados y ajustados en la sección 
6.4, podría sugerirse que las estrellas que tienen sus vientos en el régimen rápido serían HD 41117 (figura 6.12, cuya absorción "rellenada" de emisión de su perfil P Cygni no pudo ser ajustada correctamente con la solución lenta), HD 92964 (figura 6.15, similar al caso anterior, aunque tampoco pudo ser ajustada correctamente la componente en absorción por el régimen rápido).

Las candidatas a contener vientos en el régimen lento podrían ser HD 99953 (figura 6.11. la cual presenta más de una componente en absorción en $\mathrm{H} \alpha$, que pudo ser

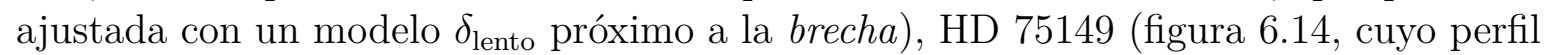
en este régimen ajusta con valores más bajos de $v_{\text {macro }}$ que los referidos en la literatura, y con buena aproximación de la tasa de pérdida de masa dada por otros autores).

No podría argumentarse en favor de uno u otro régimen respecto a HD 198478 (la figura 6.16 muestra que el ajuste logrado de $\mathrm{H} \alpha$ es pobre, sumado a la importante variabilidad en sus perfiles, Kraus et al.2015a), HD 42087 (figura 6.13, aunque el ajuste de $\mathrm{H} \alpha$ con la solución lenta es mejor, no se puede definir sólo con ese argumento) y HD 47240 (figura 6.10, que presenta ambas alas en emisión que no pudieron ser ajustadas simultáneamente con ninguna de las soluciones).

Está claro que las ideas planteadas en los párrafos anteriores son sólo sugerencias que podrían ser mejoradas por medio de un estudio más profundo de cada candidata, extendiendo el análisis a otras líneas espectrales y en otros momentos de observación, para poder establecer con mayor solidez, el régimen de viento imperante.

\subsection{La brecha en los vientos de supergigantes B}

La existencia de una brecha que separa vientos de condiciones muy diferentes en las estrellas supergigantes B, abre las posibilidades de concebir muchos escenarios que pueden favorecer la comprensión de los entornos complejos de estos objetos.

En la sección 4.3.1 se analizaron las condiciones para que un viento se encuentre a un lado o al otro de la brecha, ya sea en el régimen rápido o en el régimen lento. Básicamente, esta diferencia radica en la ionización del medio, que es considerado a través del parámetro $\delta$ de la fuerza de radiación.

\subsubsection{Transiciones entre regímenes de viento}

Si, por alguna razón a establecer, hay un cambio en las condiciones de ionización preponderantes en un viento y ese cambio es suficiente como para que producir una modificación en el parámetro $\delta$, que lleve al sistema de un régimen al otro, atravesando la brecha, habrá importantes cambios en el viento.

Esta situación puede ocurrir, en alguna instancia, debido a la evolución propia de las supergigantes B. El cambio requerido en la ionización puede tener lugar durante el tránsito del azul al rojo o del rojo al azul, en el diagrama HR (sección 1.2.2). A medida que el valor de la temperatura efectiva y con ella, las condiciones de ionización, se vayan modificando lentamente, podría esperarse que, en algún momento, se diera un cambio entre los régimenes rápido y lento, o viceversa.

En base a este argumento, podría ser posible usar estos modelos para diagnosticar en qué casos las estrellas se encuentran evolucionando hacia la región roja del diagrama HR, y en cuáles, hacia la zona azul, ya que sus vientos podrían tener distintos regímenes 
(diferentes condiciones de ionización y modos de pulsación). La determinación de qué tipo de solución se adecua al viento de una supergigante B podría ser un elemento más destinado a discernir el propio estado evolutivo de la estrella. Si la estrella evoluciona hacia temperaturas efectivas más altas, el parámetro $\delta$ podría estar incrementándose y, eventualmente, pasar de un viento rápido a uno lento.

Un mecanismo de este tipo podría contribuir, adicionalmente, a la comprensión de las fases de variabilidad en la tasa de pérdida de masa a lo largo de ciertas etapas evolutivas. Las eyecciones esporádicas de materia podrían tener como causa a este mecanismo.

El ancho de la brecha, en escala de $\delta$, también es una variable que puede tener influencia, ya que los requerimientos para un cambio de régimen serían mucho mayores en el caso de brechas "anchas".

Uno de los factores que reduce el ancho de la brecha es la tasa rotacional. A mayores valores de $\Omega$, el salto es más estrecho. Las supergigantes que tengan un valor de $\Omega \sim$ 0.6 , por ejemplo, podrán cambiar de régimen de viento con muy poco cambio en las condiciones de ionización originales.

Como se muestra en la figura 4.13(a), el cambio en los valores de $\delta$ requeridos para una transición entre dos regímenes de viento es prácticamente la misma para cualquier $\mathrm{T}_{\text {eff. }}$ Sin embargo, la transición es más probable para estrellas con baja $\mathrm{T}_{\text {eff }} \mathrm{y}$ alto $\Omega$, debido al ancho delgado de su brecha (figura 4.13(b).

Otro factor que influye en este mecanismo es el parámetro $\alpha$. Dado que los modelos con valores más pequeños de $\alpha$ producen una brecha más angosta en valores de $\delta$, los vientos más suceptibles a sufrir un cambio de régimen deberían ser aquellos que, inicialmente, posean valores bajos del parámetro $\alpha$.

De todos modos, es importante considerar que el viento de una estrella supergigante B podría tener las condiciones necesarias para estar "dentro" de la brecha, sin necesidad de transitar entre los dominios que la rodean.

\subsubsection{La brecha y la bi-estabilidad}

Los escenarios que involucran la brecha también podrían orientar la interpretación del salto de bi-estabilidad observado alrededor de $21000 \mathrm{~K}$ (sección 2.6.2). Este cambio en las condiciones de los vientos de las supergigantes B ha sido atribuido a cambios en la ionización del Fe, los que modifican de manera importante la fuerza de radiación.

Una característica interesante del salto es que el cambio experimentado en los vientos, a un lado y al otro del salto, ocurre predominantemente en la velocidad de fluido, con un factor aproximado de 2 en $\mathrm{v}_{\infty}$, estimado por Lamers et al. (1995).

Sin embargo, la correspondencia en la tasa de pérdida de masa que compensa ese cambio en velocidad (esperable según la ecuación de continuidad, ecuación 2.7) debería ser una variación de un factor 5, de acuerdo a los modelos de Vink et al. (1999). De acuerdo a Markova \& Puls (2008), el cambio en $\dot{M}$ a través del salto de bi-estabilidad, corresponde a un factor entre 0.4 y 2.5 .

De la figura 4.6, puede verse que, para el modelo base T21 la velocidad terminal en el régimen rápido es, aproximadamente, 1.5 veces la velocidad en el régimen lento. El cambio correspondiente en tasa de pérdida de masa es de 1.7 (para $\Omega=0.2$ ). Ambos 
valores se encuentran en el rango de los valores esperados a un lado y a otro del salto de bi-estabilidad. Esto sugiere que la transición de una estrella a través de la brecha, por cambios en la temperatura efectiva, podría corresponderse con el salto de bi-estabilidad.

\subsubsection{Variabilidad del viento en el corto período}

En las estrellas tempranas, una característica observacional en la escala temporal breve que ha producido mucha intriga, es la presencia de componentes angostas en absorción superpuestas a perfiles P Cygni, conocidas como DACs o discrete absorption components (sección 2.6.6). Algunos estudios han propuesto que las DACs están relacionadas con incrementos en la profundidad óptica, originadas por la presencia de plateaus en los campos de velocidad (Lamers et al. 1982).

La solución del viento obtenida con el código ZEUS-3D usando las condiciones propias de una región interior a la brecha (sección 4.6.1, figura 4.15) está en concordancia con esta clase de escenario. Una secuencia temporal de modelos que documente una transición del viento a través de la brecha podría explicar por qué las DACs evolucionan acelerando a través de los perfiles de línea hacia velocidades altas, a medida que disminuyen sus anchos.

La evolución de DACs ha sido reportada también en algunos sistemas binarios durante la mitad del período orbital (Arias et al. 2008). Para describir este fenómeno en base a los modelos presentados en este trabajo, podría construirse un escenario donde una compañera caliente irradia una región local de la superficie estelar, originando una mancha brillante. Esa región caliente produciría un cambio local en la ionización disparando, en su entorno, una modificación en el régimen del viento. Dado que la región estaría a una temperatura mayor, el viento podría adquirir un campo de velocidades compatible con una solución $\delta_{\text {lenta }}$.

Las corrientes de este viento serían más densas y lentas, capaces de generar componentes en absorciones adicionales a los perfiles $\mathrm{P}$ Cygni de las líneas espectrales, al ocultar el disco estelar.

El cambio de régimen local debería estar en fase con el movimiento orbital de la compañera, dando lugar a variaciones en los perfiles de línea, a medida que transcurre un período orbital. De esta manera, la presencia de la solución $\delta_{\text {lenta }}$ y del mecanismo de cambio podría dar soporte al modelo CIR (Co-rotating Interaction Regions), descripto por Cranmer \& Owocki (1996) para explicar las DACs. Los valores de las variables (por ejemplo, la temperatura del viento) requeridos para que este escenario sea factible aún no han sido evaluadas.

A partir de la discusión desarrollada en este capítulo sobre la viabilidad de las soluciones lentas para representar el viento de las estrellas supergigantes B, de aportar nociones alternativas para la interpretación de las discrepancias encontradas en la aplicación de la teoría estándar, y de sugerir escenarios para analizar fenómenos complejos de sus magnitudes observables, es posible reunir las conclusiones finales para este trabajo. 
7. Discusión 


\section{Capítulo 8}

\section{Perspectivas y conclusiones}

Resumen del capitulo: Se presentan las conclusiones de este trabajo y las ideas con miras a futuros estudios en el área de los vientos rotantes impulsados por radiación.

Para organizar las conclusiones de este trabajo, se revisará cada uno de los objetivos planteados inicialmente y se valorará en qué casos ha sido posible brindar una respuesta satisfactoria a cada uno de ellos, en base a los análisis desarrollados en los capítulos precedentes.

\subsection{Sobre el viento de las supergigantes B}

¿Cuál es la relación entre la fuerza de radiación que produce la aceleración del viento y las soluciones lentas y, cuáles son los dominios de cada una de ellas en el espacio de los parámetros de la misma?

¿En qué condiciones puede prevalecer una clase de solución sobre otra?

En este trabajo se exploraron las condiciones bajo las cuales pueden establecerse dos régimenes principales de velocidad para los vientos de las estrellas supergigantes $\mathrm{B}$, cada uno con características distintivas. Estos regímenes son soluciones de las ecuaciones hidrodinámicas para vientos rotantes impulsados por radiación. Los regímenes son:

- El régimen "rápido": que es la solución estándar de los vientos impulsados por radiación, y que produce vientos con mayores velocidades terminales.

- El régimen $\Omega \delta_{\text {lento }}$ o "lento": que puede clasificarse en dos sub-regímenes: el régimen $\Omega_{\text {lento }}$, que tiene lugar en estrellas con alta rotación; y el régimen $\delta_{\text {lento }}$, que surge cuando el nivel de ionización es elevado a lo largo del viento. Ambos regímenes se caracterizan por desarrollar vientos lentos y densos.

El establecimiento de cada uno de estos regímenes en el viento de una estrella con las características propias de una supergigante $\mathrm{B}$, depende de dos factores fundamentales: 
- La rotación: la incorporación de la fuerza centrífuga a la ecuación de momento del viento da como resultado a una familia distintiva de soluciones hidrodinámicas, el sub-régimen $\Omega_{\text {lento }}$, que aparece solamente cuando la tasa rotacional de la estrella es suficientemente alta $(\Omega \gtrsim 0.7)$.

- La fuerza de radiación: La teoría estándar incorpora, en la parametrización de la fuerza de radiación en líneas espectrales, información sobre la cantidad de transiciones atómicas en capacidad de absorber momento del campo de radiación a través del parámetro $k$, de la distribución de las intensidades de esas líneas, con el parámetro $\alpha$, y de las condiciones de ionización del material, por medio del parámetro $\delta$.

Excluyendo a los rápidos rotadores, si la ionización del medio es baja, se establece un viento con la estructura hidrodinámica del régimen rápido. Si la ionización es sufientemente alta (por ejemplo, debido al exceso de radiación en rayos $\mathrm{X}$ liberada por colisiones entre grumos en el viento), tiene lugar el régimen $\delta_{\text {lento }}$.

Estas potenciales estructuras hidrodinámicas son, en gran medida, independientes de la temperatura efectiva de la estrella y, por lo tanto, pueden establecerse en cualquiera de los subtipos espectrales entre las supergigantes B.

Entre los parámetros fundamentales de la estrella, el que más influye en la clase de régimen que puede adquirir un viento, es la rotación. De esta manera, el estado de ionización del medio, en sintonía con la tasa rotacional, delimitan los bordes del dominio rápido y del lento. En la medida en que las tasas rotacionales sean más elevadas, las condiciones de ionización necesarias para establecer el régimen $\delta_{\text {lento }}$ son cada vez más bajas. En el límite de altas rotaciones, sólo subsiste el régimen lento, en su sub-régimen $\Omega_{\text {lento }}, \sin$ importar la ionización del medio.

Bajo combinaciones muy especiales entre la rotación y la ionización del medio, el viento puede encontrarse en una situación intermedia, no estacionaria; estado al que se llama brecha en este trabajo. En esa circunstancia, las soluciones del viento predicen un estado híbrido, conformado por una combinación de las soluciones rápidas y lentas, con discontinuidades en el gradiente de las velocidades y en la distribución de densidad.

En base a estos resultados, se puede considerar que se han cumplido los dos primeros objetivos de esta tesis. La delimitación de los dominios de cada clase de solución hidrodinámica, junto con el relevamiento de las características propias de cada una, en función de los parámetros de la fuerza de radiación y de las propiedades fundamentales propias de la estrella modelada, representan aportes novedosos de este trabajo de tesis.

\subsection{Sobre las propiedades observadas de las super- gigantes $\mathrm{B}$}

¿Puede modelarse el viento de las estrellas supergigantes B con las soluciones lentas? ¿Son adecuadas estas soluciones? 
Para evaluar la capacidad de las soluciones lentas para representar el viento de las estrellas supergigantes B, se optó por realizar una comparación de características espectrales observadas, con las derivadas a partir de cálculos teóricos de los modelos considerados. En este trabajo se decidió probar el ajuste de líneas espectrales observadas por medio de perfiles sintéticos.

Para ajustar los perfiles teóricos a las líneas espectrales observadas, fue necesario resolver el transporte de radiación en medios en movimiento, adaptado a las condiciones hidrodinámicas de los regímenes estudiados. Con ese motivo se contribuyó a la resolución del problema en las inversiones atómicas y a su implementación en un reconocido código para el cálculo de modelos de atmósferas NLTE en expansión, dentro del estado del arte actual.

La adecuación de ese código de cálculo facilitó la obtención de perfiles sintéticos de líneas espectrales, para especies químicas relevantes en el viento de los diferentes subtipos espectrales que pertenecen al grupo de las estrellas supergigantes B.

Se eligió la línea $\mathrm{H} \alpha$ como medio apropiado para el diagnóstico de las tasas de pérdida de masa y, como un indicador secundario de las velocidades terminales del viento. Se escogió, entonces, una muestra pequeña de observaciones de supergigantes B, para intentar reproducir sus características espectrales por medio del cálculo de perfiles teóricos.

Los ajustes de los perfiles de $\mathrm{H} \alpha$ muestran que la solución lenta es adecuada para modelar los vientos de algunas estrellas supergigantes B. Estos ajustes fueron comparados con cálculos realizados para los mismos perfiles, empleando el régimen rápido. Todos los ajustes fueron realizados, directamente, a partir de la elección de diferentes valores para los parámetros de la fuerza de radiación, en un rango de valores bajos de la rotación estelar.

A pesar de la reducida extensión de la muestra observacional, puede inferirse que algunas estrellas parecen tener sus vientos dominados por regímenes semejantes al rápido, mientras que otras pueden ser mejor representadas, efectivamente, por soluciones en el régimen lento. En otros casos, sólo se pueden realizar ajustes de valor marginal, por lo que no es posible determinar qué clase de solución se les adecúa mejor a sus vientos, a partir del análisis de una única característica espectral (la línea H $\alpha$ ).

Para representar el campo de velocidades de las supergigantes B siempre se ha usado la ley $\beta$ de velocidades. Este trabajo incorpora soluciones numéricas resultantes de las ecuaciones hidrodinámicas.

En general, todos los modelos presentados en este trabajo tienden a determinar valores bajos para las velocidades terminales, los que son considerablemente menores que los valores publicados. Esto sugiere que estos modelos hidrodinámicos resultan más convenientes para representar vientos moderados alrededor de supergigantes B. Es importante notar que, con excepción de la rotación, en estos modelos no se ha hecho la incorporación de otros mecanismos físicos para obtener modelos de vientos con regímenes diferentes, más moderados o con campos de velocidades más lentos.

También, el ajuste de los perfiles observados sugiere que las tasas de pérdida de masa son menores que las obtenidas a partir de la teoría estándar de vientos impulsados por radiación. En las últimas décadas, los análisis avanzados en el estudio de los vientos han 
demostrado que la teoría de vientos impulsados por radiación tiende a sobreestimar la tasa de pérdida de masa de las estrellas tempranas. Tales cuestionamientos sugieren que los excesos por encima de los valores reales, representan un factor entre 2 y 10 veces el valor de $\dot{M}$. La causa más comúnmente atribuida para estas discrepancias en el viento, es la presencia de grumos de material o clumping. Si bien la existencia del clumping muy probablemente es real, y numerosas evidencias observacionales parecen confirmarlo; en muchas ocasiones, ha sido necesario adoptar factores de clumping muy elevados a lo largo del viento, para poder moderar las predicciones clásicas de $\dot{M}$.

Es posible que el régimen lento pueda dar cuenta, al menos parcialmente, del problema de las tasas de pérdida de masa muy elevadas. El régimen lento, podría ser una posible respuesta a la discrepancia, complementaria al fenómeno del clumpling, para resolver parcial o completamente este problema. Para evaluarlo, se requeriría un análisis más profundo sobre este tópico, con una comparación más extensiva de sus resultados y los correspondientes a trabajos recientes sobre clumping en el formalismo CAK.

Respecto a las supergigantes B con alta rotación, como es el caso de las supergigantes $\mathrm{B}[\mathrm{e}]$, es posible que la solución $\Omega_{\text {lenta }}$ sea la más adecuada para representarlas. Esas posibilidades no se han evaluado en este trabajo, ya que se debe tratar un problema en $2 \mathrm{D}$.

En base a estos análisis, en los que se estudia por primera vez la aplicación detallada de las soluciones $\delta_{\text {lenta }}$ a un conjunto de estrellas supergigantes B, es posible concluir que estas soluciones hidrodinámicas pueden ser adecuadas para modelar el viento de algunas estrellas en esta clase espectral. En estos casos, el empleo del régimen lento puede contribuir a reducir las discrepancias encontradas por la sobreestimación de las tasas de pérdida de masa.

\subsection{Sobre el aporte de las soluciones lenta a la teo- ría de vientos impulsados por radiación}

\section{¿Qué consecuencias tiene esta posibilidad sobre las relaciones más im- portantes de la teoría de vientos impulsados por radiación, tales como la relación WLR?}

La aplicación de modelos en el régimen $\delta_{\text {lento }}$ a vientos de estrellas supergigantes B ha abierto nuevas posibilidades en la exploración de las relaciones fundamentales de la teoría de vientos impulsados por radiación.

La relación entre la velocidad terminal y la velocidad de escape del viento sugiere que estos vientos son más apropiados para estrellas supergigantes B tardías o, incluso, supergigantes A, como ya lo han mostrado otros autores. Este análisis requiere un nuevo examen ya que, por ejemplo, los radios adoptados de la literatura para las estrellas consideradas aún tienen gran incerteza. Esto torna impreciso algunos resultados de este trabajo, al momento de discutir un objeto en particular.

Por otro lado, la comparación entre la relación teórica Momento del Viento - Luminosidad y los valores estimados para las estrellas de la muestra, revela que los vientos 
ajustados con la solución $\delta_{\text {lenta }}$, caen considerablemente por debajo de esta predicción. Esa discrepancia en la relación WLR ha sido encontrada también por otros autores, mostrando que es una característica común en los vientos de las estrellas supergigantes $\mathrm{B}$ intermedias. Es muy posible que esa diferencia sea causada, precisamente, por la sobreestimación de las tasas de pérdida de masa teóricas, y que, si se adopta el régimen $\delta_{\text {lento }}$, pueda obtenerse una representación más acorde de esta relación. Un análisis futuro, más extenso y profundo de este tópico, puede darle un notable impulso a la valoración de las capacidades de los vientos lentos en las supergigantes B.

Se puede concluir que la solución $\delta_{\text {lenta }}$ es apropiada para dar cuenta de algunas discrepancias encontradas en propiedades fundamentales de los vientos, tales como las que aparecen en la relación Momento del Viento - Luminosidad y en el uso de las leyes de velocidad de tipo $\beta$ con valores grandes de $\beta$, abriendo la posibilidad a realizar, mediante éstas, mediciones de distancias galácticas o extragalácticas.

La mayor relevancia de estas soluciones para dar cuenta de las diferencias observadas, se da en el caso de vientos de supergigantes B intermedias o tardías.

\section{¿Habría una manera de explorar la variabilidad de las estrellas supergi- gantes $\mathrm{B}$ en base a alternancia entre las soluciones que adopten sus vientos?}

La existencia de una brecha que delimita las soluciones rápidas de las $\delta_{\text {lenta }}$, permite proponer nuevos escenarios para facilitar la comprensión de la variabilidad observada en los vientos de las estrellas supergigantes B.

Las estrellas supergigantes pueden atravesar etapas evolutivas para las cuales, las condiciones del viento las fuercen a cruzar la brecha. Tales tránsitos por estas regiones de inestabilidad, pueden inducir cambios en la tasa de pérdida de masa o fluctuaciones en la velocidad terminal. En otras circunstancias, en una escala temporal más reducida, el paso a través de la brecha puede realizarse periódicamente, como podría ocurrir en un sistema binario cerrado. Tales irregularidades en las estructuras hidrodinámicas podrían configurar un escenario plausible para dar cuenta de fenómenos observados tales como las componentes discretas en absorción o DACs. Este marco conceptual podría aportar fundamentación a la teoría de regiones interactuantes en corrotación (modelo CIR).

Estos escenarios exigen un requisito básico: que pueda establecerse una estructura de ionización más pronunciada que la obtenida, habitualmente, a partir de las ecuaciones de balance de ionización. Entonces, es necesario que haya alguna fuente adicional de ionización, como la irradiación de rayos X.

Otro requisito es que la brecha que separa los dominios de las soluciones rápidas de las lentas sea "angosta" en el parámetro $\delta$, con un menor requerimiento de cambio del estado de ionización. Este efecto podría ser más viable en rotadores intermedios o rápidos, debido a que, para ellos, la variación en el parámetro $\delta$ necesaria para saltar la brecha, es considerablemente menor.

Una transición de vientos de esta clase para $\mathrm{T}_{\text {eff }} \gtrsim 21000 \mathrm{~K}$ podría facilitar la interpretación del salto de bi-estabilidad observado en esas estrellas. Como complemento interesante, un viento que se encuentre cursando una transición de estadios físicos co- 
mo la propuesta, podría generar, en las líneas espectrales de la estrella, componentes discretas en absorción o DACs.

\subsection{Trabajo a futuro}

En esta tesis se ha presentado la potencialidad de las soluciones lentas como un mecanismo para aproximarse a la interpretación de los vientos de las supergigantes B. Se trata de un estudio de viabilidad, cuya misión es generar nuevas expectativas en la exploración teórica del fenómeno de los vientos estelares.

En el desarrollo de este trabajo se han aplicado varias aproximaciones que serían importante mejorar. Particularmente, es necesario concentrar muchos esfuerzos para hacer que las soluciones de las ecuaciones hidrodinámicas, la ecuación de transporte de radiación y el cálculo de la fuerza de radiación, sean completamente consistentes. Estos tres ejes que posibilitan la elaboración de propiedades teóricas comparables con las observadas, deben funcionar de manera coherente y en simultáneo o, al menos, inmersos en un sistema iterativo; de manera tal que se logre un proceso autoconsistente, con el menor número posible de imposiciones desde el punto de partida.

Por otro lado, la posibilidad de encontrar una calibración más adecuada para la relación WLR, que es fundamental para la determinación espectroscópica de distancias galácticas y extragalácticas, hacen tentadora la posibilidad de una ampliación de los resultados presentados en este trabajo, usando una muestra mucho más extensa de estrellas.

Por último, la existencia de la brecha entre soluciones hidrodinámicas muy diferentes abre nuevas posibilidades para afrontar el problema de la variabilidad observada en las estrellas tempranas. La asociación de estos modelos de vientos rotantes impulsados por radiación, con otras fenomenologías como los campos magnéticos o la actividad pulsacional, puede dar nuevas claves hacia la comprensión de los vientos estelares en las estrellas masivas.

Algunas líneas de investigación mencionadas ya se han iniciado en el grupo de trabajo que comparte el autor de esta tesis, junto a sus directores, en Argentina y en Chile. Otras están a la espera de la incorporación de nuevos participantes que aporten su esfuerzo y dedicación. 
Apéndices 



\section{.1. Cálculo de la función $\mathcal{Q}$ para simetría esférica}

En el desarrollo de la aproximación de Sobolev, aplicada al cálculo de la fuerza de radiación (sección 2.2.3), se hace referencia a la igualdad 2.43 como resultado de ese producto escalar en simetría esférica. Esa ecuación se transcribe aquí para su demostración:

$$
\breve{\boldsymbol{n}} \cdot \nabla\left[\breve{\boldsymbol{n}} \cdot \boldsymbol{v}\left(\boldsymbol{r}^{\prime}\right)\right]=\mu^{2} \frac{\partial v}{\partial r}+\left(1-\mu^{2}\right) \frac{v}{r},
$$

Esta ecuación es similar, aunque con notación levemente diferente, a la forma planteada en la sección 5.1.1, donde se desarrolla la aproximación de Sobolev para resolver el transporte de radiación. En este caso, un desarrollo en serie de potencias de la velocidad permite escribir:

$$
\breve{\boldsymbol{n}} \cdot \boldsymbol{v}(\boldsymbol{r}-\breve{\boldsymbol{n}} l)=\breve{\boldsymbol{n}} \cdot \boldsymbol{v}(\boldsymbol{r})-\mathcal{Q}(\boldsymbol{r}, \breve{\boldsymbol{n}}) l,
$$

en el cual $\mathcal{Q}$ es:

$$
\mathcal{Q}(\boldsymbol{r}, \breve{\boldsymbol{n}})=\sum_{i} \sum_{j} \breve{n}_{i} \breve{n}_{j} \frac{\partial v_{i}}{\partial v_{j}}=\frac{d v_{l}}{d l},
$$

donde $\breve{n}_{i, j}$ son las direcciones del versor $\breve{\boldsymbol{n}}$. En el caso de simetría esférica, esta expresión se reduce a la forma:

$$
\mathcal{Q}=\mu^{2} \frac{\partial v}{\partial r}+\left(1-\mu^{2}\right) \frac{v}{r} .
$$

Para demostrar esa expresión para $\mathcal{Q}$, puede usarse un sistema de variables (p,z), como el empleado en la sección 5.1.3, referido al transporte de radiación en el CMF. Las relaciones básicas entre el sistema $(\mathrm{p}, \mathrm{z})$ y el sistema $(\mathrm{r}, \mu)$, con $\mu=\cos \theta$, son:

$$
\left\{\begin{array}{l}
r \equiv\left(p^{2}+z^{2}\right)^{1 / 2} \\
\mu \equiv z / r
\end{array}\right.
$$

En el sistema $(p, z)$ puede identificarse a:

$$
\left\{\begin{array}{l}
z \equiv l \\
v_{l} \equiv \mu v
\end{array}\right.
$$

Entonces:

$$
\begin{aligned}
& \left.\frac{d v}{d z}\right|_{p}=\left[\frac{d v}{d r} \frac{d r}{d z}\right]_{p}=\mu \frac{d v}{d r} \\
& \left.\frac{d \mu}{d z}\right|_{p}=\left[\frac{d\left(\frac{\left(r^{2}-p^{2}\right)^{1 / 2}}{r}\right)}{d z}\right]_{p}=\frac{d\left(\frac{z}{r}\right)}{d z}=\frac{r-\frac{d r}{d z} z}{r^{2}}=\frac{r-\mu z}{r^{2}}=\frac{1-\mu^{2}}{r}
\end{aligned}
$$

Por lo cual, finalmente resulta: 


$$
\mathcal{Q}=\frac{d v_{l}}{d l}=\frac{d(\mu v)}{d z}=\mu^{2} \frac{d v}{d r}+\left(1-\mu^{2}\right) \frac{v}{r}
$$




\section{Bibliografía}

Abbott, D. C. 1979, in IAU Symposium, Vol. 83, Mass Loss and Evolution of O-Type Stars, ed. P. S. Conti \& C. W. H. De Loore, 237-239

Abbott, D. C. 1980, ApJ, 242, 1183

Abbott, D. C. 1982a, ApJ, 263, 723

Abbott, D. C. 1982b, ApJ, 259, 282

Abbott, D. C. \& Lucy, L. B. 1985, ApJ, 288, 679

Achmad, L., Lamers, H. J. G. L. M., \& Pasquini, L. 1997, A\&A, 320, 196

Aerts, C., Christensen-Dalsgaard, J., \& Kurtz, D. W. 2010, Asteroseismology

Aerts, C., Puls, J., Godart, M., \& Dupret, M.-A. 2009, A\&A, 508, 409

Araya, I., Arcos, C., \& Curé, M. 2017, in Astronomical Society of the Pacific Conference Series, Vol. 508, The B[e] Phenomenon: Forty Years of Studies, ed. A. Miroshnichenko, S. Zharikov, D. Korčáková, \& M. Wolf, 87

Araya, I., Curé, M., \& Cidale, L. S. 2014, ApJ, 795, 81

Araya, I., Curé, M., Granada, A., \& Cidale, L. S. 2011, in IAU Symposium, Vol. 272, IAU Symposium, ed. C. Neiner, G. Wade, G. Meynet, \& G. Peters, 83-84

Arias, M. L., Sahade, J., \& Barbá, R. H. 2008, in Revista Mexicana de Astronomia y Astrofisica Conference Series, Vol. 33, Revista Mexicana de Astronomia y Astrofisica Conference Series, 118-118

Babel, J. 1996, A\&A, 309, 867

Barlow, M. J. \& Cohen, M. 1977, ApJ, 213, 737

Benaglia, P., Vink, J. S., Martí, J., et al. 2007, A\&A, 467, 1265

Bertout, C., Leitherer, C., Stahl, O., \& Wolf, B. 1985, A\&A, 144, 87

Bieging, J. H., Abbott, D. C., \& Churchwell, E. B. 1989, ApJ, 340, 518

Bjorkman, J. E. 1995, ApJ, 453, 369 
Bjorkman, J. E. 1998, in Astrophysics and Space Science Library, Vol. 233, B[e] stars, ed. A. M. Hubert \& C. Jaschek, 189

Bjorkman, J. E. \& Cassinelli, J. P. 1993, ApJ, 409, 429

Blomme, R. 1990, A\&A, 229, 513

Bouret, J.-C., Hillier, D. J., Lanz, T., \& Fullerton, A. W. 2012, A\&A, 544, A67

Bouret, J.-C., Lanz, T., \& Hillier, D. J. 2005, A\&A, 438, 301

Bresolin, F. \& Kudritzki, R. P. 2004, Origin and Evolution of the Elements, 283

Bresolin, F., Pietrzyński, G., Gieren, W., et al. 2004, ApJ, 600, 182

Butler, k. \& Giddings, J.-R. 1985, Newsletter of Analysis of Astronomical Spectra (Univ. London, 9

Carlberg, R. G. 1980, ApJ, 241, 1131

Cassinelli, J. P. 1994, Ap\&SS, 221, 277

Castor, J. I. 1979, in IAU Symposium, Vol. 83, Mass Loss and Evolution of O-Type Stars, ed. P. S. Conti \& C. W. H. De Loore, 175-189

Castor, J. I., Abbott, D. C., \& Klein, R. I. 1975, ApJ, 195, 157

Castor, J. I., Abbott, D. C., \& Klein, R. I. 1976, in Physique des Mouvements dans les Atmospheres, ed. R. Cayrel \& M. Steinberg, 363-393

Castor, J. L. 1974, MNRAS, 169, 279

Chesneau, O., Dessart, L., Kaufer, A., et al. 2011, in IAU Symposium, Vol. 272, Active OB Stars: Structure, Evolution, Mass Loss, and Critical Limits, ed. C. Neiner, G. Wade, G. Meynet, \& G. Peters, 342-347

Chesneau, O., Kaufer, A., Stahl, O., et al. 2014, A\&A, 566, A125

Chlebowski, T. \& Garmany, C. D. 1991, ApJ, 368, 241

Chlebowski, T., Harnden, Jr., F. R., \& Sciortino, S. 1989, ApJ, 341, 427

Cidale, L., Venero, R. O. J., Curé, M., \& Haucke, M. 2017, in Astronomical Society of the Pacific Conference Series, Vol. 508, The B[e] Phenomenon: Forty Years of Studies, ed. A. Miroshnichenko, S. Zharikov, D. Korčáková, \& M. Wolf, 11

Clark, J. S., Ritchie, B. W., \& Negueruela, I. 2010, A\&A, 514, A87

Clarke, D. A. 1996, ApJ, 457, 291

Clarke, D. A. 2010, ApJS, 187, 119

Conti, P. S. \& Ebbets, D. 1977, ApJ, 213, 438 
Cranmer, S. R. 1996, PhD thesis, Bartol Research Institute, University of Delaware

Cranmer, S. R. \& Owocki, S. P. 1996, ApJ, 462, 469

Crowther, P. A., Lennon, D. J., \& Walborn, N. R. 2006, A\&A, 446, 279

Curé, M. 2004, ApJ, 614, 929

Curé, M., Cidale, L., \& Granada, A. 2011, ApJ, 737, 18

Curé, M. \& Rial, D. F. 2004, A\&A, 428, 545

Curé, M., Rial, D. F., \& Cidale, L. 2005, A\&A, 437, 929

de Araujo, F. K., de Freitas-Pacheco, J. A., \& Petrini, D. 1994, MNRAS, 267, 501

De Cat, P. 2002, in Astronomical Society of the Pacific Conference Series, Vol. 259, IAU Colloq. 185: Radial and Nonradial Pulsationsn as Probes of Stellar Physics, ed. C. Aerts, T. R. Bedding, \& J. Christensen-Dalsgaard, 196

de Koter, A., Vink, J. S., \& Lamers, H. J. G. L. M. 2000, in Astronomical Society of the Pacific Conference Series, Vol. 204, Thermal and Ionization Aspects of Flows from Hot Stars, ed. H. Lamers \& A. Sapar, 135

Dessart, L. \& Owocki, S. P. 2005, A\&A, 437, 657

Donati, J.-F., Howarth, I. D., Jardine, M. M., et al. 2006, MNRAS, 370, 629

Drew, J. E. 1989, ApJS, 71, 267

Drew, J. E. 1990, ApJ, 357, 573

Drew, J. E., Hoare, M. G., \& Denby, M. 1994, MNRAS, 266, 917

Dufton, P. L., Smartt, S. J., Lee, J. K., et al. 2006, A\&A, 457, 265

Ekström, S., Georgy, C., Eggenberger, P., et al. 2012, A\&A, 537, A146

Eversberg, T., Lépine, S., \& Moffat, A. F. J. 1998, ApJ, 494, 799

Feldmeier, A. 1995, A\&A, 299, 523

Feldmeier, A. 1998, A\&A, 332, 245

Feldmeier, A., Oskinova, L., \& Hamann, W.-R. 2003, A\&A, 403, 217

Feldmeier, A., Puls, J., \& Pauldrach, A. W. A. 1997, A\&A, 322, 878

Feldmeier, A. \& Shlosman, I. 2002, ApJ, 564, 385

Fraser, M., Dufton, P. L., Hunter, I., \& Ryans, R. S. I. 2010, MNRAS, 404, 1306

Freyer, T., Hensler, G., \& Yorke, H. W. 2003, ApJ, 594, 888

Friend, D. B. \& Abbott, D. C. 1986, ApJ, 311, 701 
Fullerton, A. W., Massa, D. L., \& Prinja, R. K. 2006, ApJ, 637, 1025

Gabler, R., Gabler, A., Kudritzki, R. P., Puls, J., \& Pauldrach, A. 1989, A\&A, 226, 162

Gayley, K. G. 1995, ApJ, 454, 410

Georgy, C., Ekström, S., Eggenberger, P., et al. 2013, A\&A, 558, A103

Grady, C. A., Bjorkman, K. S., \& Snow, T. P. 1987, ApJ, 320, 376

Gray, D. F. 2005, The Observation and Analysis of Stellar Photospheres

Groenewegen, M. A. T., Lamers, H. J. G. L. M., \& Pauldrach, A. W. A. 1989, A\&A, 221,78

Gvaramadze, V. V., Langer, N., \& Mackey, J. 2012, MNRAS, 427, L50

Hamann, W.-R. \& Schmutz, W. 1987, A\&A, 174, 173

Haucke, M., Araya, I., Arcos, C., et al. 2015, in IAU Symposium, Vol. 307, IAU Symposium, ed. G. Meynet, C. Georgy, J. Groh, \& P. Stee, 104-105

Haucke, M., Cidale, L. S., Venero, R. O. J., et al. 2012, Boletin de la Asociacion Argentina de Astronomia La Plata Argentina, 55, 95

Haucke, M., Cidale, L. S., Venero, R. O. J., Curé, M., \& Kraus, M. 2017, In prep., -

Hauschildt, P. H. \& Baron, E. 1999, Journal of Computational and Applied Mathematics, 109, 41

Heger, A. \& Langer, N. 2000, ApJ, 544, 1016

Hendry, M. A., Smartt, S. J., Skillman, E. D., et al. 2008, MNRAS, 388, 1127

Herrero, A., Kudritzki, R. P., Vilchez, J. M., et al. 1992, A\&A, 261, 209

Hillier, D. J. 1987, ApJS, 63, 965

Hillier, D. J. 1991, A\&A, 247, 455

Hillier, D. J. 2012, in IAU Symposium, Vol. 282, From Interacting Binaries to Exoplanets: Essential Modeling Tools, ed. M. T. Richards \& I. Hubeny, 229-234

Hillier, D. J. \& Lanz, T. 2001, in Astronomical Society of the Pacific Conference Series, Vol. 247, Spectroscopic Challenges of Photoionized Plasmas, ed. G. Ferland \& D. W. Savin, 343

Hillier, D. J. \& Miller, D. L. 1998, ApJ, 496, 407

Hoare, M. G., Drew, J. E., \& Denby, M. 1993, MNRAS, 262, L19

Holzer, T. E. 1977, J. Geophys. Res., 82, 23 
Howarth, I. D. 2004, in IAU Symposium, Vol. 215, Stellar Rotation, ed. A. Maeder \& P. Eenens, 33

Howarth, I. D. \& Prinja, R. K. 1989, ApJS, 69, 527

Howarth, I. D., Siebert, K. W., Hussain, G. A. J., \& Prinja, R. K. 1997, MNRAS, 284, 265

Hubeny, I. 1988, Computer Physics Communications, 52, 103

Hubeny, I. \& Lanz, T. 1992, A\&A, 262, 501

Hubeny, I. \& Lanz, T. 1995, ApJ, 439, 875

Huenemoerder, D. P., Oskinova, L. M., Ignace, R., et al. 2012, ApJ, 756, L34

Hummer, D. G. \& Rybicki, G. B. 1985, ApJ, 293, 258

Humphreys, R. M. \& Davidson, K. 1994, PASP, 106, 1025

Hunter, I., Lennon, D. J., Dufton, P. L., et al. 2008, A\&A, 479, 541

Jack, D., Hauschildt, P. H., \& Baron, E. 2009, A\&A, 502, 1043

Kaper, L., Henrichs, H. F., Fullerton, A. W., et al. 1997, A\&A, 327, 281

Kaper, L., Henrichs, H. F., Nichols, J. S., et al. 1996, A\&AS, 116, 257

Kaper, L., Henrichs, H. F., Nichols, J. S., \& Telting, J. H. 1999, A\&A, 344, 231

Klein, R. I. \& Castor, J. I. 1978, ApJ, 220, 902

Kraus, M., Haucke, M., Cidale, L. S., et al. 2015a, A\&A, 581, A75

Kraus, M., Nickeler, D. H., Haucke, M., et al. 2014, in IAU Symposium, Vol. 301, Precision Asteroseismology, ed. J. A. Guzik, W. J. Chaplin, G. Handler, \& A. Pigulski, $217-220$

Kraus, M., Oksala, M. E., Cidale, L. S., et al. 2015b, ApJ, 800, L20

Krtička, J. \& Kubát, J. 2001, A\&A, 377, 175

Krtička, J. \& Kubát, J. 2016, Advances in Space Research, 58, 710

Krtička, J., Kubát, J., \& Groote, D. 2006, A\&A, 460, 145

Kubát, J., Puls, J., \& Pauldrach, A. W. A. 1999, A\&A, 341, 587

Kudritzki, R., Lennon, D. J., \& Puls, J. 1995, in Science with the VLT, ed. J. R. Walsh \& I. J. Danziger, 246

Kudritzki, R. P. 2002, ApJ, 577, 389

Kudritzki, R. P., Bresolin, F., \& Przybilla, N. 2003, ApJ, 582, L83 
Kudritzki, R. P., Mendez, R. H., Puls, J., \& McCarthy, J. K. 1997, in IAU Symposium, Vol. 180, Planetary Nebulae, ed. H. J. Habing \& H. J. G. L. M. Lamers, 64.K

Kudritzki, R. P., Pauldrach, A., \& Puls, J. 1987, A\&A, 173, 293

Kudritzki, R. P., Pauldrach, A., Puls, J., \& Abbott, D. C. 1989, A\&A, 219, 205

Kudritzki, R.-P. \& Puls, J. 2000, ARA\&A, 38, 613

Kudritzki, R. P., Puls, J., Lennon, D. J., et al. 1999, A\&A, 350, 970

Kudritzki, R. P., Simon, K. P., \& Hamann, W.-R. 1983, A\&A, 118, 245

Kudritzki, R. P., Springmann, U., Puls, J., Pauldrach, A. W. A., \& Lennon, M. 1998, in Astronomical Society of the Pacific Conference Series, Vol. 131, Properties of Hot Luminous Stars, ed. I. Howarth, 299

Kudritzki, R.-P., Urbaneja, M. A., Bresolin, F., et al. 2008, ApJ, 681, 269

Kurucz, R. L. 1979, ApJS, 40, 1

Lamers, H. J. G. \& Pauldrach, A. W. A. 1991, A\&A, 244, L5

Lamers, H. J. G. L. M. 2001, PASP, 113, 263

Lamers, H. J. G. L. M. 2013, in Astronomical Society of the Pacific Conference Series, Vol. 470, 370 Years of Astronomy in Utrecht, ed. G. Pugliese, A. de Koter, \& M. Wijburg, 97

Lamers, H. J. G. L. M. \& Cassinelli, J. P. 1999, Introduction to Stellar Winds, 452

Lamers, H. J. G. L. M., Cerruti-Sola, M., \& Perinotto, M. 1987, ApJ, 314, 726

Lamers, H. J. G. L. M., Gathier, R., \& Snow, Jr., T. P. 1982, ApJ, 258, 186

Lamers, H. J. G. L. M., Haser, S., de Koter, A., \& Leitherer, C. 1999, ApJ, 516, 872

Lamers, H. J. G. L. M. \& Leitherer, C. 1993, ApJ, 412, 771

Lamers, H. J. G. L. M. \& Rogerson, Jr., J. B. 1978, A\&A, 66, 417

Lamers, H. J. G. L. M., Snow, T. P., \& Lindholm, D. M. 1995, ApJ, 455, 269

Lamers, H. J. G. L. M. \& Snow, Jr., T. P. 1978, ApJ, 219, 504

Lamers, H. J. G. L. M., Zickgraf, F.-J., de Winter, D., Houziaux, L., \& Zorec, J. 1998, A\&A, 340, 117

Landau, L. D. \& Lifshitz, E. M. 1987, Fluid Mechanics, Vol. 6 (Pergamon Press)

Lanz, T. \& Hubeny, I. 2003, ApJS, 146, 417

Lefever, K., Puls, J., \& Aerts, C. 2007, A\&A, 463, 1093 
Lefever, K., Puls, J., Morel, T., et al. 2010, A\&A, 515, A74

Leitherer, C. 1988, ApJ, 326, 356

Leitherer, C., Robert, C., \& Drissen, L. 1992, ApJ, 401, 596

Lennon, D. J., Dufton, P. L., \& Fitzsimmons, A. 1993, A\&AS, 97, 559

Lépine, S. \& Moffat, A. F. J. 2008, AJ, 136, 548

Lucy, L. B. 1976, ApJ, 206, 499

Lucy, L. B. 1982, ApJ, 255, 278

Lucy, L. B. \& Solomon, P. M. 1970, ApJ, 159, 879

MacGregor, K. B., Hartmann, L., \& Raymond, J. C. 1979, ApJ, 231, 514

Madura, T. I., Owocki, S. P., \& Feldmeier, A. 2007, ApJ, 660, 687

Maeder, A. 2009, Physics, Formation and Evolution of Rotating Stars

Maeder, A. \& Meynet, G. 2000, A\&A, 361, 159

Maharramov, Y. M. 2013, Astronomy Reports, 57, 303

Marcolino, W. L. F., Bouret, J.-C., Martins, F., et al. 2009, A\&A, 498, 837

Markova, N. \& Puls, J. 2008, A\&A, 478, 823

Markova, N., Puls, J., Repolust, T., \& Markov, H. 2004, A\&A, 413, 693

Marlborough, J. M. \& Zamir, M. 1975, ApJ, 195, 145

Marlborough, J. M. \& Zamir, M. 1984, ApJ, 276, 706

Martins, F., Schaerer, D., Hillier, D. J., \& Heydari-Malayeri, M. 2004, A\&A, 420, 1087

Martins, F., Schaerer, D., Hillier, D. J., et al. 2005, A\&A, 441, 735

McErlean, N. D., Lennon, D. J., \& Dufton, P. L. 1999, A\&A, 349, 553

Mendez, R. H., Kudritzki, R. P., Herrero, A., Husfeld, D., \& Groth, H. G. 1988, A\&A, 190, 113

Meynet, G., Kudritzki, R.-P., \& Georgy, C. 2015, A\&A, 581, A36

Meynet, G. \& Maeder, A. 2000, A\&A, 361, 101

Meynet, G., Maeder, A., Schaller, G., Schaerer, D., \& Charbonnel, C. 1994, A\&AS, 103, 97

Mihalas, D. 1978, Stellar atmospheres /2nd edition/

Mihalas, D., Kunasz, P. B., \& Hummer, D. G. 1975, ApJ, 202, 465 
Mihalas, D., Kunasz, P. B., \& Hummer, D. G. 1976, ApJ, 206, 515

Milne, E. A. 1924, MNRAS, 84, 354

Milne, E. A. 1926, MNRAS, 86, 459

Miroshnichenko, A. S. 2006, in Astronomical Society of the Pacific Conference Series, Vol. 355, Stars with the B[e] Phenomenon, ed. M. Kraus \& A. S. Miroshnichenko, 13

Moffat, A. F. J., Drissen, L., Lamontagne, R., \& Robert, C. 1988, ApJ, 334, 1038

Mokiem, M. R., de Koter, A., Evans, C. J., et al. 2007a, A\&A, 465, 1003

Mokiem, M. R., de Koter, A., Evans, C. J., et al. 2006, A\&A, 456, 1131

Mokiem, M. R., de Koter, A., Vink, J. S., et al. 2007b, A\&A, 473, 603

Morel, T., Marchenko, S. V., Pati, A. K., et al. 2004, MNRAS, 351, 552

Morton, D. C. 1967a, ApJ, 150, 535

Morton, D. C. 1967b, ApJ, 147, 1017

Mullan, D. J. 1986, A\&A, 165, 157

Müller, P. E. \& Vink, J. S. 2008, A\&A, 492, 493

Najarro, F., Hanson, M. M., \& Puls, J. 2011, A\&A, 535, A32

Najarro, F., Kudritzki, R. P., Cassinelli, J. P., Stahl, O., \& Hillier, D. J. 1996, A\&A, 306,892

Najarro, F., Kudritzki, R.-P., Hillier, D. J., et al. 1998, in Astronomical Society of the Pacific Conference Series, Vol. 131, Properties of Hot Luminous Stars, ed. I. Howarth, 57

Nandy, K. \& Schmidt, E. G. 1975, ApJ, 198, 119

Oskinova, L. M., Hamann, W.-R., \& Feldmeier, A. 2007, A\&A, 476, 1331

Owocki, S. P. 2008, in Clumping in Hot-Star Winds, ed. W.-R. Hamann, A. Feldmeier, \& L. M. Oskinova, 121

Owocki, S. P., Castor, J. I., \& Rybicki, G. B. 1988, ApJ, 335, 914

Owocki, S. P., Cranmer, S. R., \& Blondin, J. M. 1994, ApJ, 424, 887

Owocki, S. P., Cranmer, S. R., \& Gayley, K. G. 1996, ApJ, 472, L115

Owocki, S. P., Gayley, K. G., \& Shaviv, N. J. 2004, ApJ, 616, 525

Owocki, S. P. \& Puls, J. 1999, ApJ, 510, 355 
Owocki, S. P. \& Rybicki, G. B. 1984, ApJ, 284, 337

Pamyatnykh, A. A. 1999, Acta Astron., 49, 119

Pauldrach, A. 1987, A\&A, 183, 295

Pauldrach, A., Puls, J., \& Kudritzki, R. P. 1986, A\&A, 164, 86

Pauldrach, A., Puls, J., Kudritzki, R. P., Mendez, R. H., \& Heap, S. R. 1988, A\&A, 207, 123

Pauldrach, A. W. A., Hoffmann, T. L., \& Lennon, M. 2001, A\&A, 375, 161

Pauldrach, A. W. A., Kudritzki, R. P., Puls, J., Butler, K., \& Hunsinger, J. 1994, A\&A, 283,525

Pauldrach, A. W. A. \& Puls, J. 1990, A\&A, 237, 409

Pelupessy, I., Lamers, H. J. G. L. M., \& Vink, J. S. 2000, A\&A, 359, 695

Petrenz, P. \& Puls, J. 2000, A\&A, 358, 956

Prinja, R. K., Barlow, M. J., \& Howarth, I. D. 1990, ApJ, 361, 607

Prinja, R. K. \& Massa, D. L. 2010, A\&A, 521, L55

Puls, J. 1987, A\&A, 184, 227

Puls, J. 2008, in IAU Symposium, Vol. 250, Massive Stars as Cosmic Engines, ed. F. Bresolin, P. A. Crowther, \& J. Puls, 25-38

Puls, J. \& Hummer, D. G. 1988, A\&A, 191, 87

Puls, J., Kudritzki, R., Herrero, A., et al. 1996, A\&A, 305, 171

Puls, J., Kudritzki, R.-P., Santolaya-Rey, A. E., et al. 1998, in Astronomical Society of the Pacific Conference Series, Vol. 131, Properties of Hot Luminous Stars, ed. I. Howarth, 245

Puls, J., Markova, N., \& Scuderi, S. 2008a, in Astronomical Society of the Pacific Conference Series, Vol. 388, Mass Loss from Stars and the Evolution of Stellar Clusters, ed. A. de Koter, L. J. Smith, \& L. B. F. M. Waters, 101

Puls, J., Markova, N., Scuderi, S., et al. 2006, A\&A, 454, 625

Puls, J., Petrenz, P., \& Owocki, S. P. 1999, in Lecture Notes in Physics, Berlin Springer Verlag, Vol. 523, IAU Colloq. 169: Variable and Non-spherical Stellar Winds in Luminous Hot Stars, ed. B. Wolf, O. Stahl, \& A. W. Fullerton, 131

Puls, J., Springmann, U., \& Lennon, M. 2000, A\&AS, 141, 23

Puls, J., Sundqvist, J. O., \& Rivero González, J. G. 2011, in IAU Symposium, Vol. 272, Active OB Stars: Structure, Evolution, Mass Loss, and Critical Limits, ed. C. Neiner, G. Wade, G. Meynet, \& G. Peters, 554-565 
Puls, J., Urbaneja, M. A., Venero, R., et al. 2005, A\&A, 435, 669

Puls, J., Vink, J. S., \& Najarro, F. 2008b, A\&A Rev., 16, 209

Repolust, T., Puls, J., \& Herrero, A. 2004, A\&A, 415, 349

Rivero González, J. G., Puls, J., Najarro, F., \& Brott, I. 2012, A\&A, 537, A79

Rivinius, T., Carciofi, A. C., \& Martayan, C. 2013, A\&A Rev., 21, 69

Runacres, M. C. \& Blomme, R. 1996, A\&A, 309, 544

Ryans, R. S. I., Dufton, P. L., Rolleston, W. R. J., et al. 2002, MNRAS, 336, 577

Rybicki, G. B. \& Hummer, D. G. 1978, ApJ, 219, 654

Saio, H., Georgy, C., \& Meynet, G. 2013, MNRAS, 433, 1246

Santolaya-Rey, A. E., Puls, J., \& Herrero, A. 1997, A\&A, 323, 488

Searle, S. C., Prinja, R. K., Massa, D., \& Ryans, R. 2008, A\&A, 481, 777

Silaj, J., Curé, M., \& Jones, C. E. 2014, ApJ, 795, 78

Simon, K. P., Kudritzki, R. P., Jonas, G., \& Rahe, J. 1983, A\&A, 125, 34

Simón-Díaz, S., Godart, M., Castro, N., et al. 2017, A\&A, 597, A22

Simón-Díaz, S. \& Herrero, A. 2007, A\&A, 468, 1063

Simón-Díaz, S. \& Herrero, A. 2014, A\&A, 562, A135

Snow, T. P., Lamers, H. J. G. L. M., Lindholm, D. M., \& Odell, A. P. 1994, ApJS, 95, 163

Snow, Jr., T. P. \& Morton, D. C. 1976, ApJS, 32, 429

Sobolev, V. V. 1957, Soviet Ast., 1, 678

Sobolev, V. V. 1960, Moving Envelopes of Stars. Cambridge: Harvard University Press, 1960.

Springmann, U. W. E. \& Pauldrach, A. W. A. 1992, A\&A, 262, 515

Stahl, O., Wolf, B., Aab, O., \& Smolinski, J. 1991, A\&A, 252, 693

Sterken, C. 2003, in Astronomical Society of the Pacific Conference Series, Vol. 292, Interplay of Periodic, Cyclic and Stochastic Variability in Selected Areas of the H-R Diagram, ed. C. Sterken, 437

Sternberg, A., Hoffmann, T. L., \& Pauldrach, A. W. A. 2003, ApJ, 599, 1333

Sundqvist, J. O., Owocki, S. P., \& Puls, J. 2012, in Astronomical Society of the Pacific Conference Series, Vol. 465, Proceedings of a Scientific Meeting in Honor of Anthony F. J. Moffat, ed. L. Drissen, C. Robert, N. St-Louis, \& A. F. J. Moffat, 119 
Sundqvist, J. O., Puls, J., Feldmeier, A., \& Owocki, S. P. 2011, A\&A, 528, A64

Sundqvist, J. O., Puls, J., \& Owocki, S. P. 2014, A\&A, 568, A59

Taresch, G., Kudritzki, R. P., Hurwitz, M., et al. 1997, A\&A, 321, 531

Taylor, W. D., Evans, C. J., Simón-Díaz, S., et al. 2014, MNRAS, 442, 1483

Todt, H., Sander, A., Hainich, R., et al. 2015, A\&A, 579, A75

Trundle, C., Lennon, D. J., Puls, J., \& Dufton, P. L. 2004, A\&A, 417, 217

Šurlan, B., Hamann, W.-R., Aret, A., et al. 2013, A\&A, 559, A130

van Genderen, A. M. 2001, A\&A, 366, 508

van Leeuwen, F., van Genderen, A. M., \& Zegelaar, I. 1998, A\&AS, 128, 117

Venero, R. O. J., Cidale, L. S., Curé, M., \& Haucke, M. 2017a, Enviado al Boletin de la Asociacion Argentina de Astronomia La Plata Argentina, 59

Venero, R. O. J., Curé, M., \& Cidale, L. 2016a, Boletin de la Asociacion Argentina de Astronomia La Plata Argentina, 58, 99

Venero, R. O. J., Curé, M., Cidale, L. S., \& Araya, I. 2016b, ApJ, 822, 28, Paper I

Venero, R. O. J., Curé, M., Cidale, L. S., et al. 2017b, In prep., -

Venero, R. O. J. \& Puls, J. 2002, in Revista Mexicana de Astronomia y Astrofisica Conference Series, Vol. 14, Revista Mexicana de Astronomia y Astrofisica Conference Series, ed. J. J. Claria, D. Garcia Lambas, \& H. Levato, 110

Verdugo, E., Talavera, A., \& Gómez de Castro, A. I. 1998, Ap\&SS, 263, 263

Verdugo, E., Talavera, A., \& Gómez de Castro, A. I. 1999, A\&AS, 137, 351

Villata, M. 1992, A\&A, 257, 677

Vink, J. S. 2014, ArXiv e-prints

Vink, J. S., Brott, I., Gräfener, G., et al. 2010, A\&A, 512, L7

Vink, J. S., de Koter, A., \& Lamers, H. J. G. L. M. 1999, A\&A, 350, 181

Vink, J. S., de Koter, A., \& Lamers, H. J. G. L. M. 2000, A\&A, 362, 295

von Zeipel, H. 1924, MNRAS, 84, 684

Waelkens, C. 1991, A\&A, 246, 453

Waelkens, C., Aerts, C., Kestens, E., Grenon, M., \& Eyer, L. 1998, A\&A, 330, 215

Wendker, H. J. 1987, A\&AS, 69, 87

Wenger, M., Ochsenbein, F., Egret, D., et al. 2000, A\&AS, 143, 9 
Yadav, A. P. \& Glatzel, W. 2016, MNRAS, 457, 4330

Zickgraf, F.-J., Wolf, B., Leitherer, C., Appenzeller, I., \& Stahl, O. 1986, A\&A, 163, 119

Zickgraf, F.-J., Wolf, B., Stahl, O., \& Humphreys, R. M. 1989, A\&A, 220, 206

Zickgraf, F.-J., Wolf, B., Stahl, O., Leitherer, C., \& Klare, G. 1985, A\&A, 143, 421

Zorec, J., Cidale, L., Arias, M. L., et al. 2009, A\&A, 501, 297 